\title{
Refined partition functions for open superstrings with 4,8 and 16 supercharges
}

\author{
Dieter Lüst ${ }^{\mathrm{a}, \mathrm{b}, \mathrm{c}}$, Noppadol Mekareeya ${ }^{\mathrm{b}, *}$, Oliver Schlotterer $^{\mathrm{d}}$, \\ Andrew Thomson ${ }^{\mathrm{e}}$ \\ a Arnold-Sommerfeld-Center für Theoretische Physik, Department für Physik, Ludwig-Maximilians-Universität \\ München, Theresienstraße 37, 80333 München, Germany \\ b Max-Planck-Institut für Physik (Werner-Heisenberg-Institut), Föhringer Ring 6, 80805 München, Germany \\ c CERN, Theory Group, 1211 Geneva 23, Switzerland \\ d Max-Planck-Institut für Gravitationsphysik, Albert-Einstein-Institut, 14476 Golm, Germany \\ e Theoretical Physics Group, The Blackett Laboratory, Imperial College London, SW7 2AZ, United Kingdom
}

Received 3 April 2013; received in revised form 10 June 2013; accepted 5 August 2013

Available online 20 August 2013

\begin{abstract}
We analyze the perturbative massive open string spectrum of even-dimensional superstring compactifications with four, eight and sixteen supercharges. In each of such cases, we focus on universal states that exist independently on the internal geometry and other compatification details. We analytically compute refined partition functions that count these states at each mass level. Such refined partition functions are written in a super-Poincaré covariant form, providing information on how supermultiplets transform under the little group and the R symmetry. Various asymptotic limits of the partition functions and their associated quantities, such as the leading and subleading Regge trajectories, are studied empirically and analytically. In the phenomenologically relevant case of four supercharges, the partition function can be cast into the most compact form and the asymptotic formula in the large spin limit is derived explicitly. Crown Copyright $\odot 2013$ Published by Elsevier B.V. All rights reserved.
\end{abstract}

\footnotetext{
* Corresponding author.

E-mail addresses: dieter.luest@1mu.de (D. Lüst), noppadol@mpp.mpg.de (N. Mekareeya), oliver.schlotterer@aei.mpg.de (O.Schlotterer), andrew.thomson09@imperial.ac.uk (A. Thomson).
} 


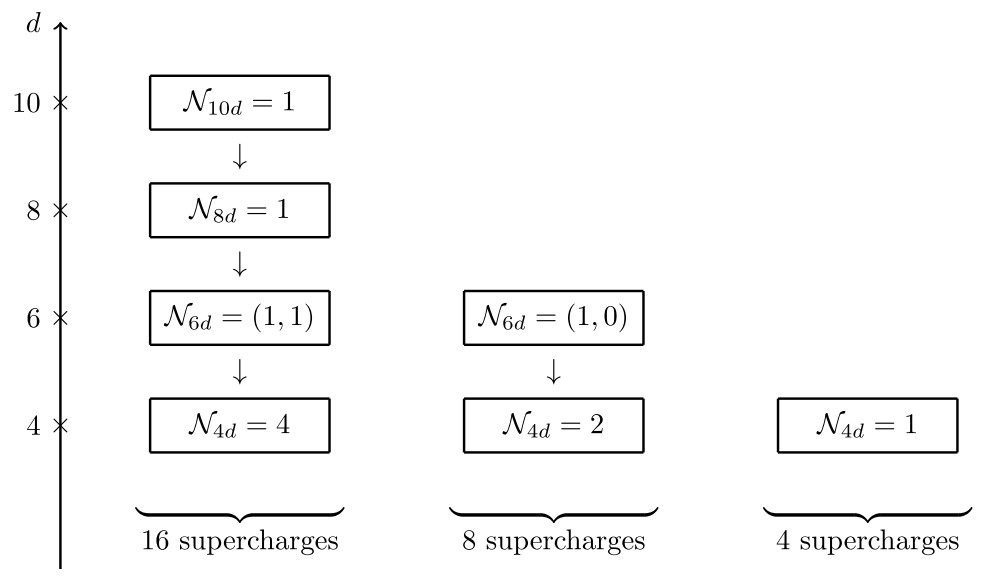

Fig. 1. Classes of superstring compactifications for which we will discuss the universal particle content. The arrows within the columns represent dimensional reduction on a $T^{2}$ torus.

\section{Introduction}

The purpose of this article is to compute the super-Poincaré covariant perturbative open superstring spectrum which is completely universal to all compactifications to 4,6 or 8 spacetime dimensions with 4, 8 or 16 supercharges. The number of such models is, of course, enormous and generic representatives have their own characteristic spectrum. Nevertheless, for each given number of supersymmetries (SUSYs), one can identify a set of physical states that exist independently on the internal geometry and any other compatification details. In this sense, one of the main aims of this work is to focus on universal statements about the spectrum in scenarios with various (even) numbers of spacetime dimensions and supercharges. The basic quantity we compute in different contexts is the number of model independent super-Poincaré multiplets of given Lorentz- and R-symmetry quantum numbers on each mass level of the superstring.

The existence of 4, 8 and 16 supercharges is compatible with various spacetime dimensions. Theories with a fixed number of supercharges are related to each other through dimensional reduction. Note that the minimum number of supercharges existing in 4, 6 and 10 dimensions is 4, 8 and 16, labeled by $\mathcal{N}_{4 d}=1, \mathcal{N}_{6 d}=(1,0)$ and $\mathcal{N}_{10 d}=1$ respectively. From each of such theories, one can therefore obtain theories with the same amount of SUSYs in lower dimensions via toroidal compactifications which preserve all the SUSYs [1]. In this paper, Kaluza-Klein and winding modes are neglected, as these depend on compactification details. Thus, determining the lower-dimensional spectra becomes a group theoretical problem of branching the associated Lorentz- and R-symmetry groups. The following Fig. 1 gives an overview of the supersymmetric theories for which we will work out the model independent subset of the open superstring spectrum.

In this paper we are providing for the first time a complete investigation of the universal massive open string states of higher spin within supersymmetric compactifications of the open Type I superstring. In particular, we will compute the partition functions of the universal open string spectra for all type I compactifications with 4, 8 and 16 preserved supercharges. Spectra of the associated Type IIA/B closed superstring theories with twice as many preserved supercharges 
can be easily inferred from our open string results through a double copy of the open string Hilbert space, that is why they will not be explicitly addressed in this paper. ${ }^{1}$

Four-dimensional superstrings subject to $\mathcal{N}_{4 d}=1$ super-Poincaré invariance are especially worth to be studied, since $\mathcal{N}_{4 d}=1$ compactifications with broken supersymmetry are expected to provide phenomenologically interesting string solutions at low energies with the spectrum of certain extensions of the supersymmetric Standard Model (see e.g. [2] for a stable low energy open string vacuum, the Standard Model $^{++}$with two Higgs fields). In addition to the light states, the knowledge of the universal massive string spectrum is also important in order to compute string scattering amplitudes of massive open string states in $\mathcal{N}_{4 d}=1$ string compactifications [3]. This task is particularly relevant, if the string scale is low compared to the Planck mass, as it is true in compactifications with large extra dimensions [4]: Both the exchange and the production of the lightest string resonances will leave measurable signatures at the LHC if the string scale is sufficiently close to the TeV range [5,6].

Apart from the phenomenological motivation, there are formal reasons to investigate scattering amplitudes among massive states: Unitarity allows to boil down the complete S-matrix of string theory down to cubic tree vertices involving any triplet of states, so it is particularly desirable to compactly represent them and to efficiently sew them together. For this programme to work in practice, the appropriate language still needs to be found, e.g. a generalization of the generating function techniques of [7] beyond the leading Regge trajectory. As an essential prerequisite, we need to get a handle on the Lorentz- and R-symmetry quantum numbers of the spectrum and make supersymmetry - the helping hand in various perturbative calculations manifest.

The refined partition functions in lower-dimensional scenarios presented in this work have the potential to contribute to such technical progress. They provide a toy laboratory with fewer states at each mass level compared to the full ten-dimensional problem of string interactions, where e.g. four-dimensional spinor helicity techniques might prove helpful in manipulating massive string amplitudes.

\subsection{Refined partition functions}

A convenient way to study the spectrum of string states is to compute a partition function that counts such states with respect to their mass levels. Since the string states transform under representations of super-Poincaré algebra, such a counting can be done in a representation theoretic way, namely the partition function can be written in terms of an infinite power series such that each power keeps track of the mass level and the coefficient of each term in the series comprises irreducible characters of the super-Poincare algebra. In this way, the symmetry of the problem is manifest in the partition function and the characters contain information on how a supermultiplet transform under the little group and the R symmetry. Moreover, knowing a partition function is equivalent to knowing how many times a given representation appears at each mass level - also known as the multiplicity. Hence, given a representation of super-Poincaré algebra, our aim is to compute its multiplicity generating function, a power series such that each power keeps track of the mass level and each coefficient are the multiplicity of this particular representation.

\footnotetext{
1 For the case of heterotic string compactifications, the charged matter fields originate from closed strings, and hence a priori one expects a different pattern of massive string states. In order to match the heterotic-type I massive string spectrum via heterotic-Type I string duality also non-perturbative states are needed.
} 
Such a way of counting of string states was already performed explicitly in [8,9] for the case of uncompactified (ten-dimensional) string theories. It has also been extensively applied to the study of moduli spaces of supersymmetric gauge theories [10-18]; in such a context the partition function is also known as the Hilbert series.

One can also view the partition function we are considering as a trace over the space of physical states. In the trace, we grade the states according to their mass levels and global charges, but not their spacetime fermion numbers. The variables used in keeping track of these levels and charges are called fugacities. The fugacities for the global charges are indeed the ones that appear in the character of a representation of the super-Poincaré algebra. In general, the partition function is therefore a multivariate function. We call the insertion of global fugacities into the trace so as to make the global symmetry manifest a refinement, and we refer to the corresponding partition function as a refined partition function. On the other hand, in order to compute the total number of states at each mass level, one can set the fugacities in the characters to unity. This amounts to computing the dimension of the corresponding representation, and we call the resulting partition function an unrefined one.

The term 'refinement' as for the insertion of the aforementioned types of fugacities has also been used recently in various papers on elliptic genera and loop amplitudes. There are various 'synonyms' that have been adopted in the literature, e.g. McKay-Thompson series [19], twining characters [20,21] and twisted elliptic genera [22-24]. We emphasize that, on the contrary to elliptic genera or other types of characters that are used in loop amplitude computations, the states that we trace over are not graded with a minus sign for spacetime fermions. ${ }^{2}$ As a result, the partition functions we are considering in this paper do not exhibit a modular invariant property. ${ }^{3}$

Open string states also carry Chan-Paton factors. The massless states and their massive excitations that arise from open strings with both endpoints attached to a stack of D branes transform in the adjoint representation of the Chan-Paton gauge group. Their character can therefore be obtained by multiplying the character discussed here by the character of the adjoint representation. ${ }^{4}$ The massive states corresponding to unoriented strings, on the other hand, transform in various representations according to the gauge symmetry (see e.g. p. 294 of [25] for further details), and the character can be computed by multiplying an appropriate character of the gauge group to our existing character at a given mass level. All partition functions computed in this paper allow for a straightforward inclusion of the Chan-Paton contributions; hence, we shall not discuss Chan-Paton factors in the subsequent.

\footnotetext{
2 To illustrate this point, let us look at the first mass level for a $10 \mathrm{~d}$ theory with 16 supercharges, there are 256 states in total (see Table 1). This number comes from (a) 44 spin two degrees of freedom and 84 three-form degrees of freedom constituting the spacetime bosonic states, and (b) 128 spin 3/2 degrees of freedom constituting the spacetime fermonic states. If we had included the grading with a minus sign for spacetime fermions into the trace, we would have a zero here. 3 To illustrate this point, we compare the unrefined partition functions presented in (5.3.37) of [25] and (9.1.14), (9.1.15) of [26]. The former is the partition function we are interested in and it is clear that such a partition function does not possess a modular invariant property. On the other hand, observe in the latter that if the grading with a minus sign for spacetime fermions is introduced in the trace, the contributions from the fermionic and bosonic excited states precisely cancel in the unrefined partition function, as exemplified in the preceding footnote.

4 Furthermore, compactifications with intersecting D branes give rise to model dependent excitations of open strings that end on different stacks of D branes [27]. These non-universal states beyond the scope of this work transform in the bifundamental representation.
} 
Table 1

The number of model independent open string states in compactifications with 4, 8 and 16 supercharges, respectively, up to mass level $\alpha^{\prime} m^{2}=9$.

\begin{tabular}{lccc}
\hline$\alpha^{\prime} m^{2}$ & \# states for 4 supercharges & \# states for 8 supercharges & \# states for 16 supercharges \\
\hline 0 & 4 & 8 & 16 \\
1 & 24 & 80 & 256 \\
2 & 104 & 512 & 2304 \\
3 & 384 & 2576 & 15360 \\
4 & 1240 & 11008 & 84224 \\
5 & 3648 & 41792 & 400896 \\
6 & 9992 & 144784 & 1711104 \\
7 & 25792 & 465856 & 6690816 \\
8 & 63392 & 1409792 & 24332544 \\
9 & 149464 & 4050112 & 83219712 \\
\hline
\end{tabular}

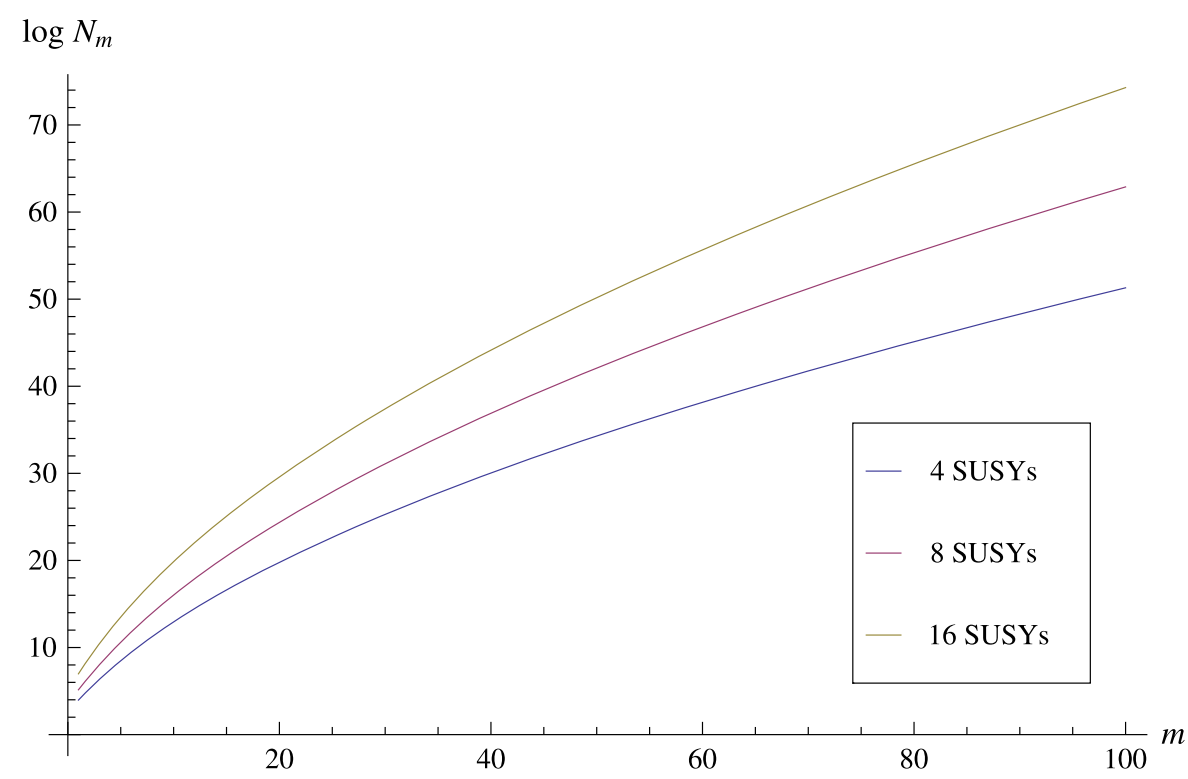

Fig. 2. The logarithmic plot of the number of states $N_{m}$ against the mass level $m$ for the case of 4 and 16 supercharges. The values of $N_{m}$ are taken from the asymptotic formulae (4.17), (5.12) and (6.10), which work well for large $m$.

\subsection{The number of universal open string states}

To give a first idea of the orders of magnitude governing the number of universal open string states at individual mass levels, the following Table 1 summarizes their numbers at low levels $\leqslant 9$ in scenarios with 4,8 and 16 supercharges, respectively. They are obtained by expanding the associated unrefined partition functions. For the cases of 4, 8 and 16 supercharges, the exact generating functions are respectively given by (4.8), (5.5), (6.6) and their asymptotics at large mass levels are respectively given by (4.17), (5.12), (6.10). Roughly speaking, the number of states increases exponentially with respect to the square root of the mass level. The plot is depicted in Fig. 2. 


\subsection{Stable patterns and Regge trajectories}

For any number of dimensions and supercharges, we can examine the multiplicities of a supermultiplet transforming under a given super-Poincaré representation. In four spacetime dimensions, such a representation contains an $S O(3)$ spin quantum number; this is half of the $S O$ (3) Dynkin label. For dimensions $d>4$, we refer to the first $S O(d-1)$ Dynkin label as 'spin' in slight abuse of terminology. This allows for the generalized notion of spin in higher dimensions. It is interesting to study the multiplicities associated with large spin quantum numbers, i.e. a large spin limit.

There are certain crucial asymptotic patterns that universally appear for families of supermultiplets, regardless of the number of dimensions and supercharges. In particular, there are certain sets of numbers that repeatedly appear at various mass levels when spins are sufficiently large (and other quantum numbers are kept fixed). As an example, it is convenient to consider Table 3 where such numbers are written in red. Since this set of numbers stabilizes in the large spin limit, we refer to it as a stable pattern. In fact, such a pattern appears not only in superstring spectra we are considering, it also does so in spectra of the bosonic and various other types of string theories as pointed out in [8]; there, the stable pattern is referred to as the leading Regge trajectory. We shall henceforth use these two terms interchangably.

Let us explore the stable pattern in more details. For a fixed sufficiently large mass level $M$, the stable pattern for a certain supermultiplet family starts appearing when the spin $j$ increases and reaches a certain value $j_{\min }(M)$. It then extends up to some maximum value $j_{\max }(M)$ where the multiplicity becomes zero for spins $j>j_{\max }(M)$. As an empirical speculation, we observe that for a sufficiently large $M$, the stable pattern appearing in the spin range $j_{\min }(M) \leqslant j \leqslant j_{\max }(M)$ occupies approximately half of the spin range $0 \leqslant j \leqslant j_{\max }(M)$ of all non-zero multiplicities. As an example, such a phenomenon is highlighted in red in each row of Table 3.

Stated differently, for a given super-Poincaré representation, the highest spin with non-zero multiplicity approximately scales linearly $j_{\max }(M) \approx\left(M-M_{0}\right)$ for large $M$ where $M_{0}$ is the mass level at which the first non-zero red number appears. The onset of the stable pattern, on the other hand, roughly follows a linear scaling, $j_{\min }(M) \approx \frac{1}{2}\left(M-M_{0}\right)$. The region of validity for the stable pattern is therefore bounded by two straight lines whose slopes have the ratio $\frac{1}{2}$. In this sense, the stable pattern gives control over the essential part of the spectrum.

In addition to the stable pattern or the leading trajectory, there is also a notion of subleading trajectories bounded by linear spin-mass relations with approximate slopes $\frac{1}{3}, \frac{1}{4}, \ldots$ We shall not go over any detail here and postpone the quantitative discussions to subsequent sections.

\subsection{Outlines and key results}

This article can be roughly divided into two parts. The first part develops the SCFT foundations for refined superstring partition functions, using conventions from Appendix A. Section 2 introduces $S O(d-1)$ covariant characters for the degrees of freedom due to the superstring oscillators from the spacetime SCFT. In order to describe compactification scenarios, the spacetime sector has to be supplemented by SCFTs describing the internal dimensions. The SCFTs discussed in Section 3 capture the universal states present in any compactification that preserves four and eight supercharges, respectively. A novel bookkeeping of internal quantum numbers is introduced to adapt the characters from the literature to the R symmetry of the spectrum.

Starting from Section 4, we proceed to the second part of this work where spacetime and internal characters are combined to super-Poincaré covariant partition functions. Universal states 
of four-dimensional $\mathcal{N}_{4 d}=1$ supersymmetric string compactifications are thoroughly investigated in Section 4: We analytically derive the stable pattern for supermultiplet multiplicities, in manifest agreement with the tabulated particle content up to mass level 25. Similarly, Section 5 is devoted to scenarios with eight supercharges - in both six and four spacetime dimensions. Finally, spectra of maximally supersymmetric open superstring theories are discussed in Section 6, a chain of dimensional reductions encompasses $d=10,8,6$ and $d=4$ compactifications.

The analysis of universal $\mathcal{N}_{4 d}=1$ supermultiplets in Section 4 enjoys the highest phenomenological relevance and provides the most compact results. Hence, the reader might want to skip Sections 2.4, 2.5 and 3.2 on higher-dimensional generalizations upon the first reading.

Let us summarize the key results in this paper below.

- The exact unrefined partition functions and asymptotic expressions for the number of states at each large mass level are given in (4.8)-(4.17), (5.5)-(5.12) and (6.6)-(6.10) for theories with four, eight and sixteen supercharges respectively. The graphs of these numbers versus the mass level are depicted in Fig. 2.

- The exact multiplicity generating functions for theories of four, eight and sixteen supercharges are respectively given in (4.61)-(4.62), (5.41) and (6.33).

- The asymptotic expressions for the multiplicity generating functions for the theory with four supercharges are presented in (4.63) and (4.64).

Even though the tools for expanding the refined partition function to any mass level are presented for all the scenarios, exact formulae for multiplicities of particular multiplets generically involve nested infinite sums. In particular, the bookkeeping of $S O(d-1)$ quantum numbers becomes increasingly difficult in $d>4$ spacetime dimensions. That is why we elaborate the phenomenologically relevant and mathematically most accessible $\mathcal{N}_{4 d}=1$ case in particular depth.

\section{The spacetime CFT in various dimensions}

This section reviews the construction of a refined partition function for the oscillator modes of the worldsheet fields $\partial X^{\mu}, \psi^{\mu}$ and fixes our notation. The worldsheet supermultiplet $\left\{\partial X^{\mu}, \psi^{\mu}\right\}$ is associated with the $d$ directions of Minkowski spacetime and carries an $S O(1, d-1)$ vector index $\mu=0,1, \ldots, d-1$. In the framework of lightcone quantization the physical spectrum is obtained from transverse oscillators $\partial X^{i=2,3, \ldots, d-1}, \psi^{i=2,3, \ldots, d-1}$ which carry charges with respect to the $\frac{1}{2}(d-2)$ Cartan generators of $S O(1, d-1)$ outside the lightcone directions. We assign a separate fugacity $y_{k}$ to each pair of $\partial X^{i}, \psi^{i}$ components (say $\left(\partial X^{2 k}, \partial X^{2 k+1}\right)$ ) such that the fugacity subscript lies in the range $1 \leqslant k \leqslant \frac{1}{2}(d-2)$. Since massive particles with $d$-dimensional timelike momentum form representations of the little group $S O(d-1)$, the dependence on Lorentz fugacities $y_{k}$ necessarily arranges into characters of the massive little group.

It is instructive to first of all study the simplest non-trivial example $d=4$ with one spacetime fugacity. The first three subsections are devoted to the $S O(3)$ covariant partition function of the four-dimensional spacetime SCFT. As we will explain in later subsections, higher-dimensional cases follow by combining several copies of $d=4$ building blocks.

\subsection{Bosonic partition function in $d=4$}

The contribution of the lightcone bosons to the refined partition function is 


$$
\begin{aligned}
\chi_{B}^{S O(3)}(q, y) & =\operatorname{PE}\left[\left([2]_{y}-1\right)\left(q+q^{2}+q^{3}+q^{4}+\cdots\right)\right]=\operatorname{PE}\left[\left([2]_{y}-1\right) \frac{q}{1-q}\right] \\
& =\prod_{n=1}^{\infty} \frac{1}{\left(1-y^{2} q^{n}\right)\left(1-y^{-2} q^{n}\right)}=\frac{1}{\left(q y^{2} ; q\right)_{\infty}\left(q y^{-2} ; q\right)_{\infty}} \\
& =-i q^{\frac{1}{12}}\left(y-y^{-1}\right) \frac{\eta(q)}{\vartheta_{1}\left(y^{2}, q\right)} .
\end{aligned}
$$

The representation [2] $y-1=y^{2}+y^{-2}$ in the plethystic exponential corresponds to the two components $\partial X^{+}, \partial X^{-}$perpendicular to the lightcone. The geometric series $\frac{q}{1-q}=q+q^{2}+$ $q^{3}+\cdots$, on the other hand, represents the infinite tower of positive frequency modes of $\partial X^{ \pm}$ which act as creation operators.

Explicitly, the first few terms in the power series of $\chi_{B}^{S O(3)}(q, y)$ can be written in terms of $S O(3)$ characters $[k]_{y}$ as

$$
\begin{aligned}
\chi_{B}^{S O(3)}(q, y)= & 1+q\left([2]_{y}-1\right)+q^{2}[4]_{y}+q^{3}\left([2]_{y}+[6]_{y}\right) \\
& +q^{4}\left([0]_{y}+2[4]_{y}+[8]_{y}\right) \\
& +q^{5}\left(2[2]_{y}+[4]_{y}+2[6]_{y}+[10]_{y}\right) \\
& +q^{6}\left(2[0]_{y}+[2]_{y}+3[4]_{y}+2[6]_{y}+2[8]_{y}+[12]_{y}\right) \\
& +q^{7}\left(4[2]_{y}+3[4]_{y}+4[6]_{y}+2[8]_{y}+2[10]_{y}+[14]_{y}\right)+\cdots
\end{aligned}
$$

From such a power series, we are motivated to rewrite (2.1) as an infinite sum of the form

$$
\chi_{B}^{S O(3)}(q, y)=\sum_{k=0}^{\infty}[k]_{y} f_{k}(q)
$$

for some function $f_{k}(q)$ which depends only on $q$ and not on $y$. The use of this form of the partition function will become clear later.

In order to do so, we rewrite (2.1) using the $q$-binomial theorem ${ }^{5}$ as

$$
\chi_{B}^{S O(3)}(q, y)=\sum_{m=0}^{\infty} \sum_{n=0}^{\infty} \frac{y^{2(m-n)}}{(q ; q)_{m}(q ; q)_{n}} q^{m+n}=: \sum_{k=0}^{\infty}[k]_{y} f_{k}(q) .
$$

Before proceeding further, let us state an identity that we are going to use many times later. From (A.5) and the residue theorem, we find that

$$
\int \mathrm{d} \mu_{S O(3)}(y) y^{m}[n]_{y}= \begin{cases}\delta_{0, n} & \text { for } m=0, \\ \frac{1}{2}\left(\delta_{|m|, n}-\delta_{|m|, n+2}\right) & \text { for } m \neq 0,\end{cases}
$$

where the Haar measure of $S O(3)$ is given by (A.9). It is clear from the absence of odd $y$ powers in (2.5) that only integer spin representations of $S O(3)$ occur. We therefore have $f_{2 k+1}(q)=0$ for all $k$, and the non-trivial coefficients to compute are $^{6}$

\footnotetext{
5 The version we use states that $\frac{1}{(z ; q)_{\infty}}=\sum_{n=0}^{\infty} \frac{z^{n}}{(q ; q)_{\infty}}$.

6 In intermediate steps, we are making use of identities like $\sum_{r=0}^{\infty} q^{r(1+p)}\left(q^{1+r} ; q\right)_{\infty}=(q ; q)_{\infty}\left(q^{1+p} ; q\right)_{\infty}$.
} 


$$
\begin{aligned}
f_{2 k}(q) & =\int \mathrm{d} \mu_{S O(3)}(y) \chi_{B}^{S O(3)}(q, y)[2 k]_{y} \\
& =\sum_{n=0}^{\infty} \frac{q^{2 n+k}}{(q ; q)_{n}(q ; q)_{n+k+1}}\left(1-q-q^{n+k+1}\right) \\
& =(q ; q)_{\infty}^{-2} \sum_{n=1}^{\infty}(-1)^{n-1}\left(1-q^{n}\right)^{2} q^{n k+\frac{1}{2} n(n-1)} .
\end{aligned}
$$

We obtain an $S O(3)$ character expansion of the bosonic partition function:

$$
\chi_{B}^{S O(3)}(q, y)=(q ; q)_{\infty}^{-2} \sum_{n=1}^{\infty}(-1)^{n-1}\left(1-q^{n}\right)^{2} \sum_{k=0}^{\infty} q^{n k+\frac{1}{2} n(n-1)}[2 k]_{y} .
$$

Note that the pattern $\sum_{n=1}^{\infty}(-1)^{n-1} q^{n k}[2 k]_{y} \ldots$ (where the ... ellipsis does not depend on $y$ and $k$ ) is described in Section 6 of [8] as an alternating sequence of additive and subtractive Regge trajectories of slope $\frac{1}{n}$. This is the source of stable patterns as described in the introduction in bosonic string theory. We will rediscover these patterns in the counting of SUSY multiplets later on.

\subsubsection{Multiplicities of representations $[2 \mathrm{~m}]$ and their asymptotics}

Let us determine the multiplicity of irreducible $S O(3)$ representations [ $2 m$ ] at each mass level. Recall the orthogonality of characters with respect to the Haar measure:

$$
\int \mathrm{d} \mu_{S O(3)}(y)[m]_{y}[n]_{y}=\delta_{m n} .
$$

From (2.8), we find that the generating function of the multiplicity of [ $2 m]$ is

$$
\begin{aligned}
M\left(\chi_{B}^{S O(3)},[2 m] ; q\right) & =\int \mathrm{d} \mu_{S O(3)}(y)[2 m]_{y} \chi_{B}^{S O(3)}(q, y) \\
& =(q ; q)_{\infty}^{-2} \sum_{n=1}^{\infty}(-1)^{n-1}\left(1-q^{n}\right)^{2} q^{\frac{1}{2} n(n-1)} q^{n m}
\end{aligned}
$$

Asymptotics as $m \rightarrow \infty$

The expression (2.10) found for multiplicity generating functions greatly simplifies in the limit $m \rightarrow \infty$ of large spin and mass level. In order to compute an asymptotic formula in this regime, we apply Laplace's method (see e.g. Section 6.7 of [28]) to our question. Since $0<q<1$, the terms in the series peak sharply near the $n=1$ term as $m \rightarrow \infty$. Therefore, it is expected that for any $\epsilon>0$

$$
M\left(\chi_{B}^{S O(3)},[2 m] ; q\right) \sim(q ; q)_{\infty}^{-2} \sum_{n=1}^{1+\lfloor\epsilon\rfloor}(-1)^{n-1}\left(1-q^{n}\right)^{2} q^{\frac{1}{2} n(n-1)} q^{n m}, \quad m \rightarrow \infty .
$$

Now let us write $n=1+t$, where $t$ is small compared with 1 . Note that

$$
q^{\frac{1}{2} n(n-1)}=1+\frac{1}{2}(\log q) t+O\left(t^{2}\right)
$$

Substituting the leading term of this power series into the right-hand side of (2.11) and extending the region of summation to $\infty$, we find that the leading behavior of $M\left(\chi_{B}^{S O(3)},[2 m] ; q\right)$ is given by 


$$
\begin{aligned}
M\left(\chi_{B}^{S O(3)},[2 m] ; q\right) & \sim(q ; q)_{\infty}^{-2} \sum_{t=0}^{\infty}(-1)^{t}\left(1-q^{t+1}\right)^{2} q^{m(t+1)} \\
& =(q ; q)_{\infty}^{-2} \frac{q^{m}(1-q)^{2}\left(1-q^{1+m}\right)}{\left(1+q^{m}\right)\left(1+q^{1+m}\right)\left(1+q^{2+m}\right)} \\
& =\left(q^{2} ; q\right)_{\infty}^{-2} \frac{q^{m}\left(1-q^{m}\right)}{\left(1+q^{m}\right)^{3}}, \quad m \rightarrow \infty .
\end{aligned}
$$

The higher order corrections can be computed by taking into account the subleading terms of (2.12). Note that the next to leading term of (2.13) is of order $O\left(q^{2 m} \log q\right)$. Thus, asymptotic formula (2.13) reproduces the exact result up to $O\left(q^{2 m-1}\right)$.

\section{Interpretation and stable pattern}

We can extract some information about bosonic string states from (2.13).

- The representation $[2 m]$ appears first time in the bosonic partition function $\chi_{B}^{S O(3)}(q, y)$ at mass level $q^{m}$.

- The multiplicities of $[2 m]$ at levels $q^{m+\ell}$, for $0 \leqslant \ell \leqslant m-1$, are independent of $m$. We refer to a set of these numbers as a stable pattern for bosonic string theory. The generating function for such a stable pattern can be determined by taking a formal limit $m \rightarrow \infty$ in (2.13):

$$
\begin{aligned}
\lim _{m \rightarrow \infty} & q^{-m} M\left(\chi_{B}^{S O(3)},[2 m] ; q\right)=\left(q^{2} ; q\right)_{\infty}^{-2}=\prod_{k=2}^{\infty}\left(1-q^{k}\right)^{-2} \\
= & 1+2 q^{2}+2 q^{3}+5 q^{4}+6 q^{5}+13 q^{6}+16 q^{7}+30 q^{8}+40 q^{9}+66 q^{10}+90 q^{11} \\
& +142 q^{12}+192 q^{13}+290 q^{14}+396 q^{15}+575 q^{16}+782 q^{17}+1112 q^{18} \\
& +1500 q^{19}+2092 q^{20}+2808 q^{21}+3848 q^{22}+5132 q^{23}+O\left(q^{24}\right)
\end{aligned}
$$

Note that terms with low orders in this power series are in agreement with the data presented in Table $6 \mathrm{~b}$ of [8].

\subsection{The NS sector in $d=4$}

Under NS boundary conditions, the worldsheet superpartners $\psi^{i}$ of the lightcone bosons contribute

$$
\begin{aligned}
f_{\mathrm{NS}}(q ; y) & =\operatorname{PE}_{F}\left[\left([2]_{y}-1\right) \frac{q^{\frac{1}{2}}}{1-q}\right] \\
& =\prod_{n=1}^{\infty}\left(1+y^{2} q^{n-1 / 2}\right)\left(1+y^{-2} q^{n-1 / 2}\right) \\
& =q^{\frac{1}{24}} \frac{\vartheta_{3}\left(y^{2}, q\right)}{\eta(q)}
\end{aligned}
$$

to the spacetime partition functions. We shall rewrite this function as an infinite sum by means of Jacobi's triple product identity (see, e.g., Subsection 19.8 of [29]):

$$
\prod_{n=1}^{\infty}\left(1-x^{2 n}\right)\left(1+x^{2 n-1} z\right)\left(1+x^{2 n-1} z^{-1}\right)=\sum_{m=-\infty}^{\infty} x^{m^{2}} z^{m}
$$


Applying identity (2.18) with $x=q^{1 / 2}$ and $z=y^{2}$ to (2.16), we obtain

$$
\begin{aligned}
f_{\mathrm{NS}}(q, y) & =(q ; q)_{\infty}^{-1} \sum_{m=-\infty}^{+\infty} y^{2 m} q^{m^{2} / 2} \\
& =(q ; q)_{\infty}^{-1} \sum_{m=0}^{\infty} q^{\frac{1}{2} m^{2}}\left(1-q^{m+\frac{1}{2}}\right)[2 m]_{y}
\end{aligned}
$$

where (2.20) can be obtained by applying (2.6) and the orthogonality of the characters to (2.19) as follows:

$$
\begin{aligned}
\int \mathrm{d} \mu_{S O(3)}(y) f_{\mathrm{NS}}(q, y)[2 k]_{y} & =(q ; q)_{\infty}^{-1}\left[\sum_{m=0}^{\infty} q^{m^{2} / 2} \delta_{m, k}-\sum_{m=-\infty}^{-1} q^{m^{2} / 2} \delta_{-m, k+1}\right] \\
& =(q ; q)_{\infty}^{-1} q^{\frac{1}{2} k^{2}}\left(1-q^{k+\frac{1}{2}}\right) .
\end{aligned}
$$

Let us combine the bosonic partition function with the NS sector contribution. Using (2.1), (2.20) and the multiplication rule $[2 m] \cdot[2 k]=\sum_{l=|k-m|}^{k+m}[2 l]$, we find that

$$
\begin{aligned}
& \chi_{\mathrm{NS}}^{S O(3)}(q, y):=\chi_{B}^{S O(3)}(q, y) f_{\mathrm{NS}}(q, y)=-i q^{1 / 8}\left(y-y^{-1}\right) \frac{\vartheta_{3}\left(y^{2}, q\right)}{\vartheta_{1}\left(y^{2}, q\right)} \\
& \quad=\frac{-1}{(q ; q)_{\infty}^{3}} \sum_{m=0}^{\infty} \sum_{n=1}^{\infty}(-1)^{n}\left(1-q^{m+\frac{1}{2}}\right)\left(1-q^{n}\right)^{2} q^{\frac{1}{2} n(n-1)+\frac{1}{2} m^{2}} \sum_{k=0}^{\infty} q^{n k} \sum_{\ell=|k-m|}^{k+m}[2 \ell] .
\end{aligned}
$$

The expression in the curly brackets $\{\cdots\}$ can be rewritten as $\sum_{k=0}^{\infty} f_{k m n}(q)[2 k]$, for some function $f_{k m n}(q)$. In order to determine this function, we use the orthogonality of characters:

$$
f_{k m n}(q)=\int \mathrm{d} \mu_{S O(3)}(y) \sum_{k^{\prime}=0}^{\infty} q^{n k^{\prime}} \sum_{\ell=\left|k^{\prime}-m\right|}^{k^{\prime}+m}[2 \ell]_{y}[2 k]_{y}=\frac{q^{n|k-m|}-q^{n(k+m+1)}}{1-q^{n}} .
$$

Therefore, we obtain

$$
\begin{aligned}
\chi_{\mathrm{NS}}^{S O(3)}(q, y)= & (q ; q)_{\infty}^{-3} \sum_{m=0}^{\infty} \sum_{n=1}^{\infty}(-1)^{n-1}\left(1-q^{m+\frac{1}{2}}\right)\left(1-q^{n}\right) q^{\frac{1}{2}\left[n(n-1)+m^{2}\right]} \\
& \times \sum_{k=0}^{\infty}\left(q^{n|k-m|}-q^{n(k+m+1)}\right)[2 k] .
\end{aligned}
$$

We emphasize that the $S O(3)$ irreducible representations with odd Dynkin labels do not appear in the partition function $\chi_{\mathrm{NS}}^{S O(3)}(q, y)$.

In terms of a power series in $q$, this can be written as

$$
\begin{aligned}
\chi_{\mathrm{NS}}^{S O(3)}(q, y)= & +q^{1 / 2}([2]-1)+q[2]+q^{3 / 2}([0]+[4]) \\
& +q^{2}([0]+2[4])+q^{5 / 2}(2[2]+[4]+[6]) \\
& +q^{3}(3[2]+[4]+2[6])+q^{7 / 2}(2[0]+2[2]+4[4]+[6]+[8]) \\
& +q^{4}(3[0]+3[2]+5[4]+2[6]+2[8])
\end{aligned}
$$




$$
\begin{aligned}
& +q^{9 / 2}([0]+7[2]+4[4]+6[6]+[8]+[10]) \\
& +q^{5}([0]+9[2]+7[4]+7[6]+2[8]+2[10])+\cdots .
\end{aligned}
$$

Setting $y=1$, we obtain the unrefined partition function

$$
\begin{aligned}
\chi_{\mathrm{NS}}^{S O(3)}(q, y=1) & =\chi_{B}^{S O(3)}(q, y) f_{\mathrm{NS}}(q, y)=\prod_{n=1}^{\infty}\left(\frac{1+q^{n-1 / 2}}{1-q^{n}}\right)^{2} \\
& =(q ; q)_{\infty}^{-3} \vartheta_{3}(1, q)=q^{-1 / 8} \frac{\vartheta_{3}(1, q)}{\eta(q)^{3}} .
\end{aligned}
$$

\subsubsection{Multiplicities of representations $[2 j]$ and their asymptotics}

Similarly to the bosonic partition function, we can read off the generating function for the multiplicities of the representations $[2 j]$ at different mass levels of the NS superstring

$$
\begin{aligned}
M\left(\chi_{\mathrm{NS}}^{S O(3)},[2 j], q\right)= & (q ; q)_{\infty}^{-3} \sum_{m=0}^{\infty}\left(1-q^{m+\frac{1}{2}}\right) q^{\frac{1}{2} m^{2}} \sum_{n=1}^{\infty}(-1)^{n-1}\left(1-q^{n}\right) q^{\frac{1}{2} n(n-1)} \\
& \times\left(q^{n|j-m|}-q^{n(j+m+1)}\right) .
\end{aligned}
$$

Asymptotics as $j \rightarrow \infty$

In this limit, we have $|j-m| \sim j-m$ for a finite $m$. Furthermore, the summand as a function of $n$ is sharply peaked near $n=1$, and so we can determine the leading behavior of the sum over $n$ using Laplace's method as follows (where $\epsilon>0$ ):

$$
\begin{aligned}
& \sum_{n=1}^{\infty}(-1)^{n-1}\left(1-q^{n}\right) q^{\frac{1}{2} n(n-1)}\left(q^{n(j-m)}-q^{n(j+m+1)}\right) \\
& \quad \sim(1-q) \sum_{n=1}^{1+\lfloor\epsilon\rfloor}\left[q^{n(j-m)}-q^{n(j+m+1)}\right] \quad \text { for } \epsilon>0 \\
& \quad \sim(1-q) \sum_{t=0}^{\infty}\left[q^{(t+1)(j-m)}-q^{(t+1)(j+m+1)}\right] \\
& \quad=q^{j-m}(1-q) \frac{1-q^{2 m+1}}{\left(1-q^{1+j+m}\right)\left(1-q^{j-m}\right)} .
\end{aligned}
$$

Therefore, we find that

$$
\begin{aligned}
M\left(\chi_{\mathrm{NS}}^{S O(3)},[2 j], q\right) & \sim(q ; q)_{\infty}^{-3} q^{j}(1-q)\left[\sum_{m=0}^{\infty} q^{-m+\frac{m^{2}}{2}} \frac{\left(1-q^{2 m+1}\right)\left(1-q^{\frac{1}{2}+m}\right)}{\left(1+q^{1+j-m}\right)\left(1+q^{j-m}\right)}\right] \\
& \sim(q ; q)_{\infty}^{-3} q^{j} \frac{1-q}{\left(1-q^{j}\right)^{2}}\left[\sum_{m=0}^{\infty} q^{\frac{1}{2}(m-1)^{2}-\frac{1}{2}}\left(1-q^{2 m+1}\right)\left(1-q^{\frac{1}{2}+m}\right)\right] \\
& =(q ; q)_{\infty}^{-3} q^{j-\frac{1}{2}}\left(\frac{1-q}{1+q^{j}}\right)^{2} \vartheta_{3}(1, q) .
\end{aligned}
$$

Note that asymptotic formula (2.30) reproduces the exact result up to the order $q^{2 j-\frac{3}{2}}$. We emphasize that the representation $[2 j]$ appears first time at mass level $q^{j-\frac{1}{2}}$. 
In [8], the individual $n$ summands of (2.28) are interpreted as an alternating sequence of additive and subtractive Regge trajectories of slope $\frac{1}{n}$. In the notation of Eq. (6.2) of that reference, the $M\left(\chi_{\mathrm{NS}}^{S O(3)},[2 j], q\right)$ are expanded as

$$
\begin{aligned}
M\left(\chi_{\mathrm{NS}}^{S O(3)},[2 j], q\right) & =q^{j} \tau_{1}^{\mathrm{NS}}(q)-q^{2 j} \tau_{2}^{\mathrm{NS}}(q)+q^{3 j} \tau_{3}^{\mathrm{NS}}(q)-\cdots \\
& =\sum_{\ell=1}^{\infty}(-1)^{\ell-1} q^{\ell j} \tau_{\ell}^{\mathrm{NS}}(q) .
\end{aligned}
$$

Setting $|j-m|=j-m$ in (2.28) leads to the following asymptotic expressions for the $\tau_{\ell}^{\mathrm{NS}}$ :

$$
\tau_{\ell}^{\mathrm{NS}}(q)=(q ; q)_{\infty}^{-3} q^{-\frac{1}{2} \ell}\left(1-q^{\ell}\right) \sum_{m=0}^{\infty} q^{\frac{1}{2}(m-\ell)^{2}}\left(1-q^{m+\frac{1}{2}}\right)\left(1-q^{2 m \ell+\ell}\right) .
$$

We will later on rediscover this trajectory structure in the counting of SUSY multiplets.

\section{The stable pattern}

The generating function of the stable pattern can be determined by projecting the sum in (2.31) to the first term (or, equivalently, by taking the limit $j \rightarrow \infty$ ):

$$
\begin{aligned}
\lim _{j \rightarrow \infty} q^{-j} M\left(\chi_{\mathrm{NS}}^{S O(3)},[2 j], q\right)= & \tau_{1}^{\mathrm{NS}}(q)=(q ; q)_{\infty}^{-3} q^{-1 / 2}(1-q)^{2} \vartheta_{3}(1, q) \\
= & \left(2+2 q+8 q^{2}+14 q^{3}+34 q^{4}+58 q^{5}+120 q^{6}\right. \\
& +204 q^{7}+378 q^{8}+632 q^{9}+1096 q^{10}+1786 q^{11} \\
& \left.+2968 q^{12}+\cdots\right)+\frac{1}{\sqrt{q}}\left(1+q+6 q^{2}+9 q^{3}+24 q^{4}\right. \\
& +42 q^{5}+88 q^{6}+151 q^{7}+287 q^{8}+480 q^{9} \\
& \left.+846 q^{10}+1388 q^{11}+2326 q^{12}+\cdots\right) .
\end{aligned}
$$

Note that terms with low orders in the power series (2.34) are in agreement with the data presented in Table $6 \mathrm{c}$ of [8].

\subsection{The $R$ sector in $d=4$}

The R sector of the worldsheet superpartners $\psi^{i}$ of the lightcone bosons contributes

$$
\begin{aligned}
f_{\mathrm{R}}(q, y) & =\left(y+y^{-1}\right) \mathrm{PE}_{F}\left[\left([2]_{y}-1\right) \frac{q}{1-q}\right] \\
& =\left(y+y^{-1}\right) \prod_{n=1}^{\infty}\left(1+y^{2} q^{n}\right)\left(1+y^{-2} q^{n}\right) \\
& =q^{-\frac{1}{12}} \frac{\vartheta_{2}\left(y^{2}, q\right)}{\eta(q)}
\end{aligned}
$$

to the spacetime partition function. Again, it will turn out to be beneficial to rewrite this function as an infinite sum. We proceed as follows. Replacing $z$ by $x z$ in (2.18), we obtain 


$$
\prod_{n=1}^{\infty}\left(1-x^{2 n}\right)\left(1+x^{2 n} z\right)\left(1+x^{2 n-2} z^{-1}\right)=\sum_{m=-\infty}^{+\infty} x^{m^{2}+m} z^{m} .
$$

Using the identity

$$
\prod_{n=1}^{\infty}\left(1+x^{2 n-2} z^{-1}\right)=\left(1+z^{-1}\right) \prod_{n=1}^{\infty}\left(1+x^{2 n} z^{-1}\right)
$$

we arrive at

$$
\left(z^{\frac{1}{2}}+z^{-\frac{1}{2}}\right) \prod_{n=1}^{\infty}\left(1+x^{2 n} z\right)\left(1+x^{2 n} z^{-1}\right)=\frac{\sum_{m=-\infty}^{+\infty} x^{m^{2}+m} z^{m+1 / 2}}{\prod_{n=1}^{\infty}\left(1-x^{2 n}\right)} .
$$

Applying identity (2.39) to (2.35) with $x=q^{1 / 2}$ and $z=y^{2}$, we have

$$
\begin{aligned}
f_{\mathrm{R}}(q, y) & =(q ; q)_{\infty}^{-1} \sum_{m=-\infty}^{+\infty} y^{2 m+1} q^{m(m+1) / 2} \\
& =(q ; q)_{\infty}^{-1} \sum_{m=0}^{\infty} q^{\frac{1}{2} m(m+1)}\left(1-q^{m+1}\right)[2 m+1]_{y} \\
& =q^{-1 / 8}(q ; q)_{\infty}^{-1} \sum_{m \in \mathbb{Z}_{\geqslant 0}+\frac{1}{2}} q^{\frac{1}{2} m^{2}}\left(1-q^{m+\frac{1}{2}}\right)[2 m]_{y},
\end{aligned}
$$

where the second equality follows from (2.6) and the orthogonality of the characters.

Let us combine the contribution from the R sector with the bosonic part. Using (2.1) and (2.35), we find that

$$
\begin{aligned}
\chi_{\mathrm{R}}^{S O(3)}(q, y):= & \chi_{B}^{S O(3)}(q, y) f_{\mathrm{R}}(q, y)=-i\left(y-y^{-1}\right) \frac{\vartheta_{2}\left(y^{2}, q\right)}{\vartheta_{1}\left(y^{2}, q\right)} \\
= & q^{-\frac{1}{8}}(q ; q)_{\infty}^{-3} \sum_{m \in \mathbb{Z}_{\geqslant 0}+\frac{1}{2}}^{\infty} \sum_{n=1}^{\infty}(-1)^{n-1}\left(1-q^{m+\frac{1}{2}}\right)\left(1-q^{n}\right) q^{\frac{1}{2}\left[n(n-1)+m^{2}\right]} \\
& \times \sum_{k=0}^{\infty}\left(q^{n|k-m|}-q^{n(k+m+2)}\right)[2 k+1]
\end{aligned}
$$

This resembles (2.25) up to a shift in the summations over $m, k$ by $\pm \frac{1}{2}$. We emphasize that $S O$ (3) irreducible representation with even Dynkin labels do not appear in the R-sector partition function $\chi_{\mathrm{R}}^{S O(3)}(q, y)$.

In terms of a power series, this partition function can be written as

$$
\begin{aligned}
\chi_{\mathrm{R}}^{S O(3)}(q, y)= & {[1]+2[3] q+2([1]+[3]+[5]) q^{2}+(4[1]+4[3]+4[5]+2[7]) q^{3} } \\
& +(6[1]+10[3]+8[5]+4[7]+2[9]) q^{4} \\
& +(12[1]+18[3]+16[5]+10[7]+4[9]+2[11]) q^{5} \\
& +(22[1]+32[3]+30[5]+22[7]+10[9]+4[11]+2[13]) q^{6} \\
& +(36[1]+58[3]+56[5]+40[7]+24[9] \\
& +10[11]+4[13]+2[15]) q^{7}+\cdots .
\end{aligned}
$$


Setting $y=1$, we obtain the unrefined partition function

$$
\chi_{\mathrm{R}}^{S O(3)}(q, y=1)=2 \prod_{n=1}^{\infty}\left(\frac{1+q^{n}}{1-q^{n}}\right)^{2}=q^{-\frac{1}{8}}(q ; q)_{\infty}^{-3} \vartheta_{2}(1, q)=\frac{\vartheta_{2}(1, q)}{\eta(q)^{3}}
$$

\subsubsection{Multiplicities of representations $[2 j+1]$ and their asymptotics}

The generating function for the multiplicities of the representations $[2 j+1]$ at different mass levels are

$$
\begin{aligned}
& M\left(\chi_{\mathrm{R}}^{S O(3)},[2 j+1], q\right) \\
& =q^{-\frac{1}{8}}(q ; q)_{\infty}^{-3} \sum_{m=0}^{\infty}\left(1-q^{m+1}\right) q^{\frac{1}{2}\left(m+\frac{1}{2}\right)^{2}} \sum_{n=1}^{\infty}(-1)^{n-1}\left(1-q^{n}\right) q^{\frac{1}{2} n(n-1)} \\
& \quad \times\left(q^{n|j-m|}-q^{n(j+m+2)}\right)
\end{aligned}
$$

in close analogy to (2.28). In fact, one can obtain the above formula by shifting $m \rightarrow m+\frac{1}{2}$ and $j \rightarrow j+\frac{1}{2}$ in (2.28) and multiply by an overall factor $q^{-\frac{1}{8}}$.

Asymptotics as $j \rightarrow \infty$

Similarly to the NS sector, we find that the leading behavior of $M\left(\chi_{\mathrm{R}}^{S O(3)},[2 j+1], q\right)$ is

$$
\begin{aligned}
& M\left(\chi_{\mathrm{R}}^{S O(3)},[2 j+1], q\right) \\
& \sim q^{-\frac{1}{8}}(q ; q)_{\infty}^{-3} q^{j+\frac{1}{2}} \frac{1-q}{\left(1-q^{j}\right)^{2}}\left[\sum_{m=0}^{\infty} q^{\frac{1}{2}\left(m-\frac{1}{2}\right)^{2}-\frac{1}{2}}\left(1-q^{2 m+2}\right)\left(1-q^{m+1}\right)\right] \\
& =(q ; q)_{\infty}^{-3} q^{j-\frac{1}{8}}\left(\frac{1-q}{1+q^{j}}\right)^{2} \vartheta_{2}(1, q) .
\end{aligned}
$$

Note that the representation $[2 j+1]$ appears first time at mass level $q^{j}$ and the asymptotic formula reproduces the exact result up to the order $q^{2 j-1}$.

Also the multiplicity generating functions of the Ramond sector are suitable for an expansion in terms of Regge trajectories:

$$
\begin{aligned}
M\left(\chi_{\mathrm{R}}^{S O(3)},[2 j+1], q\right) & =q^{j} \tau_{1}^{\mathrm{R}}(q)-q^{2 j} \tau_{2}^{\mathrm{R}}(q)+q^{3 j} \tau_{3}^{\mathrm{R}}(q)-\cdots \\
& =\sum_{\ell=1}^{\infty}(-1)^{\ell-1} q^{\ell j} \tau_{\ell}^{\mathrm{R}}(q) .
\end{aligned}
$$

The $|j-m|=j-m$ asymptotics yield the following expressions for the $\ell$ 'th Ramond trajectory $\tau_{\ell}^{\mathrm{R}}$ :

$$
\tau_{\ell}^{\mathrm{R}}(q)=(q ; q)_{\infty}^{-3} q^{-\frac{1}{8}}\left(1-q^{\ell}\right) \sum_{m=\frac{1}{2}}^{\infty} q^{\frac{1}{2}(m-\ell)^{2}}\left(1-q^{m+\frac{1}{2}}\right)\left(1-q^{2 m \ell+\ell}\right) .
$$

\section{The stable pattern}

The generating function of the stable pattern can be determined by taking the limit $j \rightarrow \infty$ : 


$$
\begin{aligned}
\lim _{j \rightarrow \infty} & q^{-j} M\left(\chi_{\mathrm{R}}^{S O(3)},[2 j+1], q\right)=\tau_{1}^{\mathrm{R}}(q)=(q ; q)_{\infty}^{-3} q^{-1 / 8}(1-q)^{2} \vartheta_{2}(1, q) \\
= & 2+4 q+10 q^{2}+24 q^{3}+48 q^{4}+96 q^{5}+184 q^{6}+336 q^{7} \\
& +600 q^{8}+1048 q^{9}+1784 q^{10}+2984 q^{11}+4912 q^{12}+7952 q^{13} \\
& +12704 q^{14}+20048 q^{15}+31256 q^{16}+O\left(q^{17}\right) .
\end{aligned}
$$

Note that terms with low orders in the power series (2.50) are in agreement with the data presented in Table $6 \mathrm{~d}$ of [8].

\subsection{Bosonic partition function in $d>4$}

The bosonic partition function in $d=2 n+2$ spacetime dimensions can be written as

$$
\chi_{B}^{S O(2 n+1)}(q, y)=\mathrm{PE}\left[\left([1,0, \ldots, 0]_{y}^{S O(2 n+1)}-1\right) \frac{q}{1-q}\right],
$$

where $\boldsymbol{y}=\left(y_{1}, \ldots, y_{n}\right)$ and the character of the vector representation $[1,0, \ldots, 0]$ of $S O(2 n+1)$ is given by (A.6). The $2 n=d-2$ summands in $[1,0, \ldots, 0]_{y}^{S O(2 n+1)}-1=\sum_{k=1}^{n}\left(y_{k}^{2}+y_{k}^{-2}\right)$ reflect the $\partial X^{i}$ components outside the lightcone. Using (A.8), we see that this choice of character allows us to write

$$
\begin{aligned}
\chi_{B}^{S O(2 n+1)}(q, \boldsymbol{y}) & =\operatorname{PE}\left[\frac{q}{1-q} \sum_{k=1}^{n}\left([2]_{y_{k}}-1\right)\right] \\
& =\prod_{A=1}^{n} \chi_{B}^{S O(3)}\left(y_{A}\right) .
\end{aligned}
$$

Observe that the $(2 n+2)$-dimensional partition function is simply a product of $n$ copies of the four-dimensional partition function. From (2.8), we have

$$
\begin{aligned}
& \chi_{B}^{S O(2 n+1)}(q, \boldsymbol{y}) \\
& \quad=(q ; q)_{\infty}^{-2 n} \sum_{\boldsymbol{n} \in \mathbb{Z}_{+}^{n}} \sum_{\boldsymbol{k} \in \mathbb{Z}_{\geqslant 0}^{n}} \prod_{A=1}^{n}(-1)^{n_{A}-1}\left(1-q^{n_{A}}\right)^{2} q^{n_{A} k_{A}+\frac{1}{2} n_{A}\left(n_{A}-1\right)}\left[2 k_{A}\right]_{y_{A}}
\end{aligned}
$$

with $\mathbb{Z}_{+}$denoting the set of positive integers and $\mathbb{Z}_{\geqslant 0}=\mathbb{Z}_{+} \cup\{0\}$. For our purpose of resolving the $S O(2 n+1)$ content of the partition function, the aim is to rewrite $(2.53)$ in the form

$$
\chi_{B}^{S O(2 n+1)}(q, \boldsymbol{y})=\sum_{\lambda_{1} \geqslant \cdots \geqslant \lambda_{n} \geqslant 0}\left(\lambda_{1}, \ldots, \lambda_{n}\right)_{y} G_{\lambda_{1}, \ldots, \lambda_{n}}^{B, S O(2 n+1)}(q),
$$

where the summations run over highest weight vectors $\lambda:=\left(\lambda_{1}, \ldots, \lambda_{n}\right) \in \mathbb{Z}^{n}$ subject to inequalities $\lambda_{1} \geqslant \cdots \geqslant \lambda_{n} \geqslant 0$, see Appendix A for the conversion rule to Dynkin label notation $\left[a_{1}, \ldots, a_{n}\right]$. Since $(2.51)$ involves only the vector representation and the plethystic exponential generates symmetrizations of the representation, there is no spinor representation of $S O(2 n+1)$ appearing in $\chi_{B}^{S O(2 n+1)}(q, \boldsymbol{y})$; therefore,

$$
G_{\lambda_{1}+\frac{1}{2}, \ldots, \lambda_{n}+\frac{1}{2}}^{B, S O(2 n+1)}(q)=0, \quad \lambda_{k} \in \mathbb{Z} .
$$

In general, $G_{\lambda_{1}, \ldots, \lambda_{n}}^{B, S O(2 n+1)}(q)$ can be interpreted as a generating function for the multiplicities of the $S O(2 n+1)$ representation $\left(\lambda_{1}, \ldots, \lambda_{n}\right)$ in the bosonic string partition function. 


\subsubsection{Some useful relations between $S O(2 n+1)$ and $S O(3)$ representations}

In order to obtain compact formulae for the multiplicity generating functions $G_{\lambda_{1}, \ldots, \lambda_{n}}^{B, S O(2 n+1)}(q)$, we have to convert the $S O(3)$ character products in (2.53) into a basis of $\left(\lambda_{1}, \ldots, \lambda_{n}\right)_{y}$, i.e. we have to find the $\Delta$ coefficients in the basis transformation

$$
\prod_{A=1}^{n}\left[2 k_{A}\right]_{y_{A}}=\sum_{\lambda_{1} \geqslant \cdots \geqslant \lambda_{n} \geqslant 0} \Delta\left(\lambda_{1}, \ldots, \lambda_{n} ; 2 k_{1}, \ldots, 2 k_{n}\right)\left(\lambda_{1}, \ldots, \lambda_{n}\right)_{y} .
$$

In general, according to (5.10) of [8], it can be shown that the coefficients in this basis transformation are given by

$$
\begin{aligned}
\Delta\left(\lambda_{1}, \ldots, \lambda_{n} ; 2 k_{1}, \ldots, 2 k_{n}\right) & :=\int \mathrm{d} \mu_{S O(2 n+1)}(y)\left(\lambda_{1}, \ldots, \lambda_{n}\right) y \prod_{A=1}^{n}\left[2 k_{A}\right]_{y_{A}} \\
& =\frac{1}{n !} \sum_{\sigma_{1}, \sigma_{2} \in S_{n}} \operatorname{sgn}\left(\sigma_{2}\right) \prod_{A=1}^{n} \theta_{\left|\lambda_{A}-A+\sigma_{2}(A)\right|}^{2 n+\lambda_{A}-A-\sigma_{2}(A)}\left(k_{\sigma_{1}(A)}\right) \\
& =\frac{1}{n !} \sum_{\sigma \in S_{n}} \operatorname{det}\left(\theta_{\left|\lambda_{A}-A+B\right|}^{2 n+\lambda_{A}-A-B}\left(k_{\sigma(A)}\right)\right)_{A, B=1}^{n}
\end{aligned}
$$

where the function $\theta_{m}^{n}(k)$ is defined as

$$
\theta_{m}^{n}(k)= \begin{cases}1 & \text { if } m \leqslant k \leqslant n \\ 0 & \text { otherwise }\end{cases}
$$

Note that for spinorial representations $\left(\lambda_{1}+\frac{1}{2}, \ldots, \lambda_{n}+\frac{1}{2}\right),(2.57)$ vanishes identically:

$$
\begin{gathered}
\Delta\left(\lambda_{1}+\frac{1}{2}, \ldots, \lambda_{n}+\frac{1}{2} ; 2 k_{1}, \ldots, 2 k_{n}\right)=0, \\
\forall \lambda \in \mathbb{Z}^{n} \text { and } \lambda_{1} \geqslant \cdots \geqslant \lambda_{n} \geqslant 0 .
\end{gathered}
$$

Thus, Eq. (2.57) implies the following expansion rule for $S O(3)$ character products in terms of $S O(2 n+1)$ characters in Dynkin label notation:

$$
\begin{aligned}
& \prod_{A=1}^{n}\left[2 k_{A}\right]_{y_{A}} \\
& \quad=\sum_{\ell \in \mathbb{Z}_{\geqslant 0}^{n}}\left[\ell_{1}, \ldots, \ell_{n-1}, 2 \ell_{n}\right]_{y} \Delta\left(2 k_{1}, \ldots, 2 k_{n} ; \ell_{1}+\ell_{2}+\cdots+\ell_{n}, \ell_{2}+\cdots+\ell_{n}, \ldots, \ell_{n}\right) .
\end{aligned}
$$

The inverse decomposition formula for an integer spin representation follows from the $S O(2 n+1)$ Haar measure (A.10):

$$
\begin{aligned}
& {\left[\ell_{1}, \ldots, \ell_{n-1}, 2 \ell_{n}\right]_{\boldsymbol{y}}} \\
& \quad=\frac{1}{\rho(\boldsymbol{y})} \sum_{\boldsymbol{k} \in \mathbb{Z}_{\geqslant 0}^{n}} \prod_{A=1}^{n}\left[2 k_{A}\right]_{y_{A}} \Delta\left(\ell_{1}+\ell_{2}+\cdots+\ell_{n}, \ell_{2}+\cdots+\ell_{n}, \ldots, \ell_{n} ; 2 k_{1}, \ldots, 2 k_{n}\right),
\end{aligned}
$$


where $\rho(\boldsymbol{y})$ is defined as in (A.11) and $\boldsymbol{\ell}=\left(\ell_{1}, \ldots, \ell_{n}\right) \in \mathbb{Z}_{\geqslant 0}^{n}$.

Similarly, one can convert spinorial $S O(3)$ character products to $S O(2 n+1)$ characters via

$$
\begin{aligned}
\Delta & \left(\lambda_{1}, \ldots, \lambda_{n} ; 2 k_{1}+1, \ldots, 2 k_{n}+1\right) \\
& :=\int \mathrm{d} \mu_{S O(2 n+1)}(y)\left(\lambda_{1}, \ldots, \lambda_{n}\right) y \prod_{A=1}^{n}\left[2 k_{A}+1\right]_{y_{A}} \\
& =\frac{1}{n !} \sum_{\sigma \in S_{n}} \operatorname{det}\left(\theta_{\left|\lambda_{A}-A+B\right|}^{2 n+\lambda_{A}-A-B}\left(k_{\sigma(A)}+\frac{1}{2}\right)\right)_{A, B=1}^{n} .
\end{aligned}
$$

For integer spin representations of $S O(2 n+1),(2.62)$ vanishes identically:

$$
\Delta\left(\lambda_{1} \ldots, \lambda_{n} ; 2 k_{1}+1, \ldots, 2 k_{n}+1\right)=0, \quad \forall \lambda \in \mathbb{Z}^{n} \text { and } \lambda_{1} \geqslant \cdots \geqslant \lambda_{n} \geqslant 0 .
$$

We thus have the following decomposition for products of spinorial $S O(3)$ characters

$$
\begin{aligned}
\prod_{A=1}^{n}\left[2 k_{A}+1\right]_{y_{A}}= & \sum_{\ell \in \mathbb{Z}_{\geqslant 0}^{n}}\left[\ell_{1}, \ldots, \ell_{n-1}, 2 \ell_{n}+1\right]_{y} \\
& \times \Delta\left(2 k_{1}+1, \ldots, 2 k_{n}+1 ; \ell_{1}+\ell_{2}+\cdots+\frac{1}{2} \ell_{n}, \ell_{2}+\cdots\right. \\
& \left.+\frac{1}{2} \ell_{n}, \ldots, \frac{1}{2} \ell_{n}\right),
\end{aligned}
$$

with inverse

$$
\begin{aligned}
{\left[\ell_{1}, \ldots, \ell_{n-1}, 2 \ell_{n}+1\right]_{\boldsymbol{y}}=} & \frac{1}{\rho(\boldsymbol{y})} \sum_{\boldsymbol{k} \in \mathbb{Z}_{\geqslant 0}^{n}} \prod_{A=1}^{n}\left[2 k_{A}+1\right]_{y_{A}} \\
& \times \Delta\left(\ell_{1}+\ell_{2}+\cdots+\ell_{n}+\frac{1}{2}, \ell_{2}+\cdots+\ell_{n}+\frac{1}{2}, \ldots, \ell_{n}\right. \\
& \left.+\frac{1}{2} ; 2 k_{1}+1, \ldots, 2 k_{n}+1\right) .
\end{aligned}
$$

\subsubsection{Generating function for the multiplicities}

According to (2.53), the bosonic spacetime partition function in $2 n+2$ dimensions depends on Lorentz fugacities through the factor

$$
\begin{aligned}
& \sum_{k_{1}, \ldots, k_{n} \geqslant 0} \Delta\left(\lambda_{1}, \ldots, \lambda_{n} ; 2 k_{1}, \ldots, 2 k_{n}\right) q^{n_{1} k_{1}+\cdots+n_{n} k_{n}} \\
& =\sum_{k_{1}, \ldots, k_{n} \geqslant 0} \operatorname{det}\left(\theta_{\left|\lambda_{A}-A+B\right|}^{2 n+\lambda_{A}-A-B}\left(k_{A}\right)\right)_{A, B=1}^{n} q^{n_{1} k_{1}+\cdots+n_{n} k_{n}} \\
& =\operatorname{det}\left(\sum_{k_{A} \geqslant 0} \theta_{\left|\lambda_{A}-A+B\right|}^{2 n+\lambda_{A}-A-B}\left(k_{A}\right) q^{n_{A} k_{A}}\right)_{A, B=1}^{n}
\end{aligned}
$$

Let us apply this to (2.53) to compute $G_{\lambda_{1}, \ldots, \lambda_{n}}^{B, S O(2 n+1)}(q)$. For $\lambda_{1} \geqslant \cdots \geqslant \lambda_{n} \geqslant n-1$, the argument in the absolute value is non-negative and so 


$$
\begin{aligned}
& \sum_{k_{1}, \ldots, k_{n} \geqslant 0} \Delta\left(\lambda_{1}, \ldots, \lambda_{n} ; 2 k_{1}, \ldots, 2 k_{n}\right) q^{n_{1} k_{1}+\cdots+n_{n} k_{n}} \\
& =\prod_{A=1}^{n} q^{n_{A}\left(\lambda_{A}-A+1\right)} \prod_{1 \leqslant B<C \leqslant n}\left(q^{n_{C}}-q^{n_{B}}\right)\left(1-q^{n_{C}+n_{B}}\right)
\end{aligned}
$$

for $\lambda_{1} \geqslant \cdots \geqslant \lambda_{n} \geqslant n-1$.

It is pointed out by [8] and can be checked directly that the contribution from $\lambda_{n}<n-1$ to the bosonic string partition function is zero. Therefore, we have

$$
\begin{aligned}
G_{\lambda_{1}, \ldots, \lambda_{n}}^{B, S O(2 n+1)}(q)= & (q ; q)_{\infty}^{-2 n} \sum_{n \in \mathbb{Z}_{+}^{n}} \prod_{A=1}^{n}(-1)^{n_{A}-1}\left(1-q^{n_{A}}\right)^{2} q^{n_{A}\left(\lambda_{A}-A+1\right)+\frac{1}{2} n_{A}\left(n_{A}-1\right)} \\
& \times \prod_{1 \leqslant B<C \leqslant n}\left(q^{n_{C}}-q^{n_{B}}\right)\left(1-q^{n_{C}+n_{B}}\right),
\end{aligned}
$$

for all $\lambda_{1}, \ldots, \lambda_{n} \in \mathbb{Z}$ and $\lambda_{1} \geqslant \cdots \geqslant \lambda_{n} \geqslant 0$.

2.5. The contributions from the NS and $R$ sectors in $d>4$

The contribution from the NS sector can be obtained by taking a product of $n$ copies of (2.25):

$$
\begin{aligned}
\chi_{\mathrm{NS}}^{S O(2 n+1)}(q, \boldsymbol{y}) \\
=\prod_{A=1}^{n} \chi_{\mathrm{NS}}^{S O(3)}\left(q ; y_{A}\right) \\
=(q ; q)_{\infty}^{-3 n} \sum_{\boldsymbol{m} \in \mathbb{Z}_{\geqslant 0}^{n}} \sum_{\boldsymbol{n} \in \mathbb{Z}_{+}^{n}} \prod_{A=1}^{n}(-1)^{n_{A}+1}\left(1-q^{m_{A}+\frac{1}{2}}\right)\left(1-q^{n_{A}}\right) q^{\frac{1}{2}\left[n_{A}\left(n_{A}-1\right)+m_{A}^{2}\right]} \\
\quad \times \sum_{\boldsymbol{k} \in \mathbb{Z}_{\geqslant 0}^{n}}^{\infty} \prod_{A=1}^{n}\left(q^{n_{A}\left|k_{A}-m_{A}\right|}-q^{n_{A}\left(k_{A}+m_{A}+1\right)}\right)\left[2 k_{A}\right]_{y_{A}} .
\end{aligned}
$$

Similarly for the contribution from the R sector, the product of $n$ copies of (2.42):

$$
\begin{aligned}
\chi_{\mathrm{R}}^{S O(2 n+1)}(q, \boldsymbol{y}) \\
=\prod_{A=1}^{n} \chi_{\mathrm{R}}^{S O(3)}\left(q ; y_{A}\right) \\
=q^{-\frac{n}{8}}(q ; q)_{\infty}^{-3 n} \sum_{\boldsymbol{m} \in \mathbb{Z}_{\geqslant 0}^{n}} \sum_{\boldsymbol{n} \in \mathbb{Z}_{+}^{n}} \prod_{A=1}^{n}(-1)^{n_{A}+1}\left(1-q^{m_{A}+1}\right)\left(1-q^{n_{A}}\right) q^{\frac{1}{2}\left[n_{A}\left(n_{A}-1\right)+\left(m_{A}+\frac{1}{2}\right)^{2}\right]} \\
\quad \times \sum_{\boldsymbol{k} \in \mathbb{Z}_{\geqslant 0}^{n}} \prod_{A=1}^{n}\left(q^{n_{A}\left|k_{A}-m_{A}\right|}-q^{n_{A}\left(k_{A}+m_{A}+2\right)}\right)\left[2 k_{A}+1\right]_{y_{A}} .
\end{aligned}
$$

The unrefined partition functions can be written as 

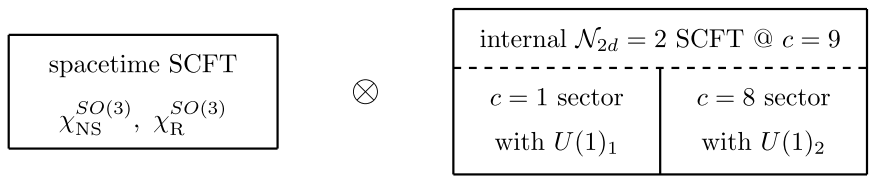

Fig. 3. Universal SCFT ingredients of $\mathcal{N}_{4 d}=1$ scenarios.

$$
\begin{aligned}
& \chi_{\mathrm{NS}}^{S O(2 n+1)}\left(q,\left\{y_{i}=1\right\}\right)=q^{-n / 8} \frac{\vartheta_{3}(1, q)^{n}}{\eta(q)^{3 n}}, \\
& \chi_{\mathrm{R}}^{S O(2 n+1)}\left(q,\left\{y_{i}=1\right\}\right)=\frac{\vartheta_{2}(1, q)^{n}}{\eta(q)^{3 n}} .
\end{aligned}
$$

\section{Internal SCFTs}

The SCFT description of four- and six-dimensional string compactifications with $\mathcal{N}_{4 d}=1$, $\mathcal{N}_{4 d}=2$ or $\mathcal{N}_{6 d}=(1,0)$ spacetime SUSY comprises universal sectors with enhanced $\mathcal{N}_{2 d}=2,4$ worldsheet SUSY [30-33]. The purpose of this section is to collect the associated charged characters, starting from the expressions given in [34,35] but adapting the dependence on fugacities $s, x$ and $z$ of the internal symmetries to the R symmetries of the spectrum.

\section{1. $\mathcal{N}_{2 d}=2$ worldsheet superconformal algebra at $c=9$}

The internal SCFT universal to any four-dimensional string compactification with $\mathcal{N}_{4 d}=1$ spacetime SUSY enjoys $\mathcal{N}_{2 d}=2$ worldsheet SUSY. The resulting model independent partition function receives contributions from characters of the $\mathcal{N}_{2 d}=2$ superconformal algebra with central charge $c=9$. Its representations are characterized by the conformal weight $h$ and the $U$ (1) charge $\ell$ of their highest weight state. The representations needed to describe $\mathcal{N}_{4 d}=1$ compactifications have $(h, \ell)=(0,0)$ in the NS sector and $(h, \ell)=\left(\frac{3}{8}, \frac{3}{2}\right)$ in the R sector.

The $\mathcal{N}_{2 d}=2$ SCFT at $c=9$ can be split into two decoupled sectors, each of which enjoys a $U(1)$ symmetry. The first one carries central charge $c=1$ and can be completely bosonized; let us denote the $U(1)$ occurring in this sector by $U(1)_{1}$ and its $h=1$ current by $\mathcal{J}_{1}$. In addition, there exists a second decoupled sector with $c=8$ which involves conformal primaries $g^{ \pm}$of weight $\frac{4}{3}$, see e.g. [33]. It enjoys an independent $U(1)_{2}$ under which the $g^{ \pm}$have opposite charges. The $c=9$ supercurrent can be split into two components $G_{\text {int }}^{ \pm}$that carry opposite charges under both $U(1)_{1}$ and $U(1)_{2}$ and factorize into conformal primaries of both sectors. The following Fig. 3 summarizes the decoupling SCFT ingredients.

Spacetime symmetries are generated by BRST invariant $h=1$ SCFT operators, and it turns out that only the current $\mathcal{J}_{1}+\mathcal{J}_{2}$ associated with the diagonal subgroup $S\left(U(1)_{1} \times U(1)_{2}\right)$ is BRST closed. Hence, only $S\left(U(1)_{1} \times U(1)_{2}\right)$ can take the role of the $U(1)_{R}$ symmetry of the spectrum. Accordingly, we have to define the charged internal character with respect to the diagonal current $\mathcal{J}_{1}+\mathcal{J}_{2}$ to see the $U(1)_{R}$ at the level of the partition function. ${ }^{7}$

\footnotetext{
7 We cannot give a local representation of $\mathcal{J}_{2}$ in terms of the $g^{ \pm}$fields from the $c=8$ sector, but we can make its existence plausible through an analogy: The currents of the $S O(d)$ Lorentz symmetry schematically read $\psi^{\mu} \psi^{\nu}+$ $X^{[\mu} \partial X^{\nu]}$. Even though $X^{\mu}$ itself is not a conformal field involved in the construction of the spectrum, the product $X^{[\mu} \partial X^{\nu]}$ is inevitable to form a BRST invariant completion of the $h=1$ primary $\psi^{\mu} \psi^{\nu}$. The addition of $X^{[\mu} \partial X^{\nu]}$ for the sake of BRST closure is the spacetime SCFT analogue of the $\mathcal{J}_{2}$ current.
} 
We denote the fugacity for charge under the $S\left(U(1)_{1} \times U(1)_{2}\right) \cong U(1)_{R}$ subgroup by $s$. On the level of the charged characters, this leads to a different fugacity dependence compared to $(3.15)^{8}$ of [34] where the internal charge is defined through the $\mathcal{J}_{1}$ eigenvalue rather than the $\mathcal{J}_{1}+\mathcal{J}_{2}$ eigenvalue. ${ }^{9}$ For instance, the supercurrent components are products of operators from both sectors, so $G_{\text {int }}^{ \pm}$are charged under both $U(1)_{1}$ and $U(1)_{2}$ but neutral under the diagonal subgroup $S\left(U(1)_{1} \times U(1)_{2}\right)$. The $\chi_{\mathrm{NS}, \mathrm{R}}^{S O(3)}$ factors in the following character formulae are due to the oscillator modes of the stress energy tensor, the internal current and the supercurrents. $U(1)_{R}$ neutrality of the latter forbids an $s$ dependence at this point and sets the second argument of the $\chi_{\mathrm{NS}, \mathrm{R}}^{\mathrm{SO}(3)}$ characters to unity.

\subsubsection{The NS sector}

The internal character in this sector is given by

$$
\begin{aligned}
\chi_{\mathrm{NS}, h=0, \ell=0}^{\mathcal{N}_{2 d}=2, c=9}(q ; s)= & (1-q) \chi_{\mathrm{NS}}^{S O(3)}(q, 1) \sum_{p \in \mathbb{Z}} \frac{q^{p^{2}+p-\frac{1}{2}} s^{2 p}}{\left(1+q^{p-\frac{1}{2}}\right)\left(1+q^{p+\frac{1}{2}}\right)} \\
= & (q ; q)_{\infty}^{-3}(1-q) \vartheta_{3}(1, q) \sum_{p \in \mathbb{Z}} \frac{q^{p^{2}+p-\frac{1}{2}} s^{2 p}}{\left(1+q^{p-\frac{1}{2}}\right)\left(1+q^{p+\frac{1}{2}}\right)} \\
= & 1+q+\left(2+s_{2}\right) q^{3 / 2}+\left(3+s_{2}\right) q^{2}+\left(4+s_{2}\right) q^{5 / 2}+\left(6+2 s_{2}\right) q^{3} \\
& +\left(10+4 s_{2}\right) q^{7 / 2}+\left(15+6 s_{2}\right) q^{4}+\left(20+8 s_{2}\right) q^{9 / 2} \\
& +\left(28+12 s_{2}\right) q^{5}+\left(42+19 s_{2}+s_{4}\right) q^{11 / 2} \\
& +\left(59+27 s_{2}+2 s_{4}\right) q^{6}+\left(78+36 s_{2}+2 s_{4}\right) q^{13 / 2} \\
& +\left(107+51 s_{2}+3 s_{4}\right) q^{7}+O\left(q^{15 / 2}\right),
\end{aligned}
$$

where we have introduced the notation

$$
s_{n}= \begin{cases}s^{n}+s^{-n}, & n>0, \\ 1, & n=0\end{cases}
$$

to compactly represent the fugacity dependence.

The unrefined internal character (i.e. setting $s$ to unity) can be rewritten in terms of modular functions as follows:

$$
\chi_{\mathrm{NS}, h=0, \ell=0}^{\mathcal{N}_{2 d}=2, c=9}(q ; s=1)=q^{1 / 8} \frac{\vartheta_{3}(1, q)}{\eta(q)^{3}}\left[\vartheta_{3}\left(1, q^{2}\right)-q^{1 / 4} \vartheta_{2}\left(1, q^{2}\right)\right] .
$$

\footnotetext{
8 The R sector analogue of the NS character (3.15) is not explicitly displayed in [34] but must be inferred through spectral flow.

9 The author of [34] denotes by $z$ the fugacity of charge under $U(1)_{1}$. For us, it makes sense to rescale the units of internal charge by $3 / 2$ which amounts to the correspondence $s \leftrightarrow z^{3 / 2}$ (in addition to the aforementioned inclusion of $\mathcal{J}_{2}$ ). Moreover, the character in (3.15) of [34] is defined as the trace over $q^{L_{0}-c / 24}$, with $c=9$, instead of $q^{L_{0}}$. The reason we consider the latter is because we are dealing with critical string theories, and so the total central charge of all matter and (super) ghost sectors taken together vanishes; this explains the presence of $q^{-9 / 24}$ factor in (3.15) of [34] but not in (3.1).
} 


\subsubsection{The R sector}

The internal character in this sector is given by

$$
\begin{aligned}
\chi_{\mathrm{R}, h=3 / 8, \ell=3 / 2}^{\mathcal{N}_{2 d}=2, c=9}(q ; s)= & (1-q) \chi_{\mathrm{R}}^{S O(3)}(q, 1) \sum_{p \in \mathbb{Z}} \frac{q^{p^{2}-1} s^{2 p-1}}{\left(1+q^{p}\right)\left(1+q^{p-1}\right)} \\
= & (q ; q)_{\infty}^{-3}(1-q) \vartheta_{2}(1, q) \sum_{p \in \mathbb{Z}} \frac{q^{p^{2}-\frac{9}{8}} s^{2 p-1}}{\left(1+q^{p}\right)\left(1+q^{p-1}\right)} \\
= & s_{1}+2 s_{1} q+6 s_{1} q^{2}+\left(2 s_{3}+14 s_{1}\right) q^{3}+\left(4 s_{3}+30 s_{1}\right) q^{4} \\
& +\left(10 s_{3}+62 s_{1}\right) q^{5}+\left(24 s_{3}+122 s_{1}\right) q^{6}+\left(50 s_{3}+230 s_{1}\right) q^{7} \\
& +O\left(q^{8}\right) .
\end{aligned}
$$

The unrefined internal character can be rewritten in terms of modular functions as

$$
\chi_{\mathrm{R}, h=3 / 8, \ell=3 / 2}^{\mathcal{N}_{2 d}=2, c=9}(q ; s=1)=q^{-1 / 4} \frac{\vartheta_{2}(1, q)}{\eta(q)^{3}}\left[\vartheta_{2}\left(1, q^{2}\right)-q^{1 / 4} \vartheta_{3}\left(1, q^{2}\right)\right] .
$$

\subsubsection{Some features}

Let us discuss some properties of the above internal characters.

- The units of $U(1)_{R}$ charge are normalized such that all integer powers of $s$ occur. According to the infinite sums within (3.1) and (3.4), even powers $s_{2} p$ firstly occur along with $q^{p^{2}+p-1 / 2}$, i.e. in the NS sector at mass level $p^{2}+p-1$. Odd powers $s_{2 p-1}$ of the $U(1)_{R}$ fugacity, on the other hand, firstly show up at power $q^{p^{2}-5 / 8}$, i.e. in the R sector at mass level $p^{2}-1 .^{10}$

- The unrefined internal R character (3.5) can be derived from the NS counterpart (3.3) by exchanging $\vartheta_{2}$ and $\vartheta_{3}$ and multiplying by an overall factor $q^{-3 / 8}$.

- In contrast to their cousins in [34], the charged characters (3.1) and (3.4) of the NS and R sector are not related by spectral flow because the internal fugacity $s$ is defined through the $U(1)_{R}$ symmetry current $\mathcal{J}_{1}+\mathcal{J}_{2}$ and not through the bosonizable $U(1)_{1}$ current $\mathcal{J}_{1}$.

- Both of the unrefined internal characters (3.3) and (3.5) are not modular invariant. This can be seen from the modular transformation $q \mapsto \tilde{q}=e^{-2 \pi i / \tau}$,

$$
\begin{aligned}
& \vartheta_{2}(1, \tilde{q})=\vartheta_{4}(1, q) \sqrt{-i \tau}, \quad \eta(\tilde{q})=\eta(q) \sqrt{-i \tau}, \\
& \vartheta_{3}(1, \tilde{q})=\vartheta_{3}(1, q) \sqrt{-i \tau} .
\end{aligned}
$$

10 The onset of the $s_{m}$ at $q$ power $q^{\frac{1}{4} m^{2}+\frac{1}{2} m+\text { const }}$ might seem counterintuitive in view of the bosonized operators $e^{ \pm \frac{i}{2} \sqrt{3} m H}$ (with $H$ a free boson) which contribute $s_{m} q^{\frac{3}{8} m^{2}}$ to the character. The mismatch between the $q$ exponents $\frac{1}{4} m^{2}+\frac{1}{2} m$ and $\frac{3}{8} m^{2}$ is caused by the fact that generic contributions to $s_{m}$ at fixed $U(1)_{R} \cong S\left(U(1)_{1} \times U(1)_{2}\right)$ charge stem from composite fields with both $U(1)_{1}$ and $U(1)_{2}$ charges. The operator of lowest conformal weight along with some $s_{m \geqslant 3}$ is charged under both $U(1)_{1}$ and $U(1)_{2}$. Since the internal fugacities in [34] only count $U(1)_{1}$ charges and are insensitive to $U(1)_{2}$, the leading $q$ power associated with some $s_{m}$ in the characters of the reference can be directly traced back to the aforementioned operators $e^{ \pm \frac{i}{2} \sqrt{3} m H}$. 

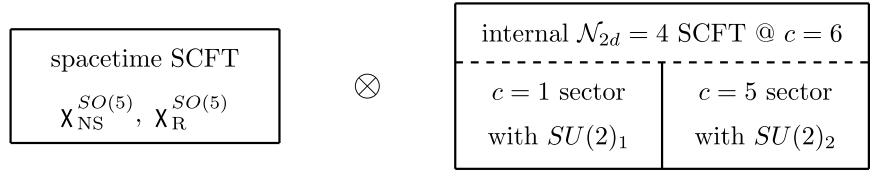

Fig. 4. Universal SCFT ingredients of $\mathcal{N}_{6 d}=(1,0)$ scenarios.

\section{2. $\mathcal{N}_{2 d}=4$ worldsheet superconformal algebra at $c=6$}

The existence of eight supercharges in four or six-dimensional spacetime implies that the universal part of the internal SCFT contains a sector with central charge $c=6$, enhanced $\mathcal{N}_{2 d}=$ 4 worldsheet SUSY and $S U(2)$ Kac-Moody symmetry at level 1 . The $c=6$ representations contributing to the NS sector and R sector of $\mathcal{N}_{4 d}=2$ and $\mathcal{N}_{6 d}=(1,0)$ spectra are characterized by values $(h, \ell)=(0,0)$ and $(h, \ell)=\left(\frac{1}{4}, \frac{1}{2}\right)$, respectively, of the conformal weight $h$ and the spin $\ell$ with respect to the $S U(2)$ Kac-Moody symmetry.

The $c=6$ SCFT is governed by $\mathcal{N}_{2 d}=4$ worldsheet SUSY and $S U(2)$ Kac-Moody symmetry at level $k=1$. In the notation of [33], the supercurrent components are built from two spin fields $\lambda^{1,2}$ of conformal weight $\frac{1}{4}$ which form a doublet under the $S U(2)_{1}$ Kac-Moody currents $\mathcal{J}^{A=1,2,3}$ and additional weight $\frac{5}{4}$ fields $g_{1,2}$ which decouple from the $\mathcal{J}^{A}$. The $g_{1,2}$ form a doublet under another $S U(2)_{2}$ which is embedded into the SCFT sector decoupling from $\mathcal{J}^{A}$. Fig. 4 summarizes the mutually decoupling SCFT sectors involved in $\mathcal{N}_{6 d}=(1,0)$ compactifications:

We shall use charged characters in the following where the fugacity $r$ is defined with respect to the diagonal subgroup within the two decoupling $S U(2)$ 's acting on the $\lambda^{1,2}$ and $g_{1,2}$ doublets. In other words, the insertion into the character trace is the BRST invariant sum of the two $S U(2)_{1,2}$ Cartan generators associated with the $S U(2)_{R}$ symmetry of the spectrum. This makes sure that the diagonal component $\lambda^{1} g_{1}+\lambda^{2} g_{2}$ of the supercurrent is a singlet of the diagonal $S U(2)$, as required by the BRST invariance. The character formulae (21) and (22) in $[35]^{11}$ are therefore slightly modified in their $r$ dependence.

\subsubsection{The NS sector}

The internal character in this sector is given by

$$
\begin{aligned}
\chi_{\mathrm{NS}, h=0, \ell=0}^{\mathcal{N}_{2 d}=4, c=6}(q ; r)= & \chi_{\mathrm{NS}}^{S O(3)}(q, 1) \sum_{m \in \mathbb{Z}} q^{\frac{1}{2} m^{2}+\frac{1}{4}} r^{2 m} \frac{q^{m-\frac{1}{2}}-r^{-2}}{1+q^{m-\frac{1}{2}}} \\
= & (q ; q)_{\infty}^{-3} \vartheta_{3}(1, q) \sum_{k=0}^{\infty}[2 k]_{r} \frac{(1-q)\left(1-q^{k+\frac{1}{2}}\right)}{\left(1+q^{k-\frac{1}{2}}\right)\left(1+q^{k+\frac{3}{2}}\right)} q^{\frac{1}{2} k^{2}+k-\frac{1}{2}} \\
= & {[0]_{r}+[2]_{r} q+\left([2]_{r}+[0]_{r}\right) q^{3 / 2}+\left([2]_{r}+2[0]_{r}\right) q^{2} } \\
& +\left(2[2]_{r}+2[0]_{r}\right) q^{5 / 2}+\left(4[2]_{r}+2[0]_{r}\right) q^{3} \\
& +\left([4]_{r}+5[2]_{r}+4[0]_{r}\right) q^{7 / 2}+\left(2[4]_{r}+6[2]_{r}+7[0]_{r}\right) q^{4} \\
& +\left(2[4]_{r}+10[2]_{r}+8[0]_{r}\right) q^{9 / 2}+\left(3[4]_{r}+16[2]_{r}+9[0]_{r}\right) q^{5} \\
& +\left(6[4]_{r}+21[2]_{r}+15[0]_{r}\right) q^{11 / 2}+\left(9[4]_{r}+27[2]_{r}+23[0]_{r}\right) q^{6}
\end{aligned}
$$

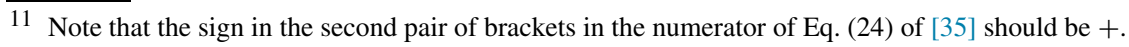




$$
\begin{aligned}
& +\left(12[4]_{r}+39[2]_{r}+27[0]_{r}\right) q^{13 / 2} \\
& +\left([6]_{r}+17[4]_{r}+56[2]_{r}+33[0]_{r}\right) q^{7}+O\left(q^{15 / 2}\right) .
\end{aligned}
$$

The unrefined internal character for the NS sector can be written as

$$
\chi_{\mathrm{NS}, h=0, \ell=0}^{\mathcal{N}_{2 d}=4, c=6}(q ; r=1)=q^{1 / 8} \frac{\vartheta_{3}(1, q)^{2}}{\eta(q)^{3}}\left[1-2 i q^{1 / 8} \mu\left(\frac{1+\tau}{2}, \tau\right)\right],
$$

where $\mu(u, \tau)$ is an Appell-Lerch sum defined in (A.20); for our purpose, we have ${ }^{12}$

$$
\mu\left(\frac{1+\tau}{2}, \tau\right)=-\frac{i}{\vartheta_{3}(1, q)} \sum_{m \in \mathbb{Z}} \frac{q^{\frac{1}{2} m^{2}-\frac{1}{8}}}{1+q^{m-\frac{1}{2}}},
$$

where we have used the fact that $\vartheta_{1}\left(e^{2 \pi i(1+\tau) / 2}, q\right)=q^{-1 / 8} \vartheta_{3}(1, q)$.

\subsubsection{The $R$ sector}

The internal character in this sector is given by

$$
\begin{aligned}
\chi_{\mathrm{R}, h=\frac{1}{4}, \ell=\frac{1}{2}}^{\mathcal{N}_{2 d}=4, c=6}(q ; r)= & \chi_{\mathrm{R}}^{S O(3)}(q, 1) \sum_{m \in \mathbb{Z}} r^{2 m+1} \frac{q^{m}-r^{-2}}{1+q^{m}} q^{\frac{1}{2} m^{2}+\frac{1}{2} m} \\
= & q^{-\frac{1}{8}}(q ; q)_{\infty}^{-3} \vartheta_{2}(1, q) \sum_{k=0}^{\infty}[2 k+1]_{r} \frac{(1-q)\left(1-q^{k+1}\right)}{\left(1+q^{k}\right)\left(1+q^{k+2}\right)} q^{\frac{1}{2} k^{2}+\frac{3}{2} k} \\
= & {[1]_{r}+2[1]_{r} q+\left(2[3]_{r}+4[1]_{r}\right) q^{2}+\left(4[3]_{r}+10[1]_{r}\right) q^{3} } \\
& +\left(10[3]_{r}+20[1]_{r}\right) q^{4}+\left(2[5]_{r}+22[3]_{r}+38[1]_{r}\right) q^{5} \\
& +\left(6[5]_{r}+44[3]_{r}+72[1]_{r}\right) q^{6}+\left(14[5]_{r}+86[3]_{r}+130[1]_{r}\right) q^{7} \\
& +O\left(q^{8}\right) .
\end{aligned}
$$

The unrefined internal character for the $\mathrm{R}$ sector can be written as

$$
\begin{aligned}
\chi_{\mathrm{R}, h=\frac{1}{4}, \ell=\frac{1}{2}}^{\mathcal{N}_{2 d}=4, c=6}(q ; r=1) & =\frac{\vartheta_{2}(1, q)}{\eta(q)^{3}} \sum_{m \in \mathbb{Z}}\left(\frac{q^{m}-1}{1+q^{m}}\right) q^{\frac{1}{2} m(m+1)} \\
& =\frac{\vartheta_{2}(1, q)}{\eta(q)^{3}} \sum_{m \in \mathbb{Z}}\left[\left(1-\frac{2}{1+q^{m}}\right) q^{\frac{1}{2} m(m+1)}\right] \\
& =q^{-1 / 8} \frac{\vartheta_{2}(1, q)^{2}}{\eta(q)^{3}}\left[1-2 i q^{1 / 8} \mu(1 / 2, \tau)\right],
\end{aligned}
$$

where we have ${ }^{13}$

$$
\mu(1 / 2, \tau)=-\frac{i}{\vartheta_{2}(1, q)} \sum_{m \in \mathbb{Z}} \frac{q^{\frac{1}{2} m(m+1)}}{1+q^{m}},
$$

where we have used the fact that $\vartheta_{1}(-1, q)=\vartheta_{2}(1, q)$.

\footnotetext{
12 This function is also closely related to the function $h_{3}(q)$ introduced in [35-37].

13 This function is also closely related to the function $h_{2}(q)$ introduced in [35-37].
} 
Some features

- According to Appendix A, characters $[n]_{r}$ of $S U(2)_{R}$ follow the same highest weight notation as for $S O(3)$, i.e. we have $[1]_{r}=r+r^{-1}$ for the fundamental representation and $[n]_{r}=\sum_{k=-n / 2}^{+n / 2} r^{2 k}$ in the general spin $n / 2$ case. Again, the infinite sum representations allow to read off the lowest level where individual $S U(2)_{R}$ representations contribute: Integer spin representations $[2 k]_{r}$ firstly occur at power $q^{k^{2} / 2+k-1 / 2}$, i.e. at mass level ${ }^{14}$ $\left\lfloor k^{2} / 2+k-1 / 2\right\rfloor$. Spinorial representations $[2 k+1]_{r}$, on the other hand, firstly show up at $q^{k^{2} / 2+3 k / 2-1 / 4}$, i.e. at mass level $k(k+3) / 2 .^{15}$

- Observe that the unrefined internal characters in both NS and R sectors involve Appell-Lerch sums, which are mock modular forms. Since the characters and are holomorphic in $q$, it is immediate that they are not modular invariant. Also, as before in the $\mathcal{N}_{2 d}=2$ SCFT, the relation between NS and R characters through spectral flow is absent due to the adaption of the internal fugacity to the $S U(2)_{R}$ symmetry.

\section{Spectrum in $\mathcal{N}_{4 d}=1$ supersymmetric compactifications}

This section opens up the main body of this work where the SCFT ingredients introduced so far are applied to counting universal super-Poincaré multiplets in the perturbative string spectrum. ${ }^{16}$ We start with the phenomenologically relevant and mathematically most tractable $\mathcal{N}_{4 d}=1$ supersymmetric scenario. Its SCFT description requires the internal sector with enhanced $\mathcal{N}_{2 d}=2$ worldsheet SUSY introduced in Section 3.1, independently on the compactification details. The BRST invariant completion of the internal current takes the role of the $U(1)_{R}$ symmetry generator. Lorentz quantum numbers enter through the partition functions (2.25) and (2.42) of the spacetime SCFT for the $\partial X^{\mu}$ and $\psi^{\mu}$ oscillators, expressed in terms of characters of the massive little group $S O(3)$ in four dimension.

The universal part of the $\mathcal{N}_{4 d}=1$ spectrum is built from both spacetime oscillators and internal operators. On the level of its partition function $\chi^{\mathcal{N}_{4 d}=1}(q ; y, s)$, this amounts to forming a GSO projected product of NS and R characters from the spacetime- and internal SCFT, see (3.1) and (3.4) for the latter. In a power series expansion in $q$, the coefficient of the $n$ 'th power $q^{n}$ comprises characters for the $\mathcal{N}_{4 d}=1$ super-Poincaré multiplets occurring at the $n$ 'th mass level with $m^{2}=n / \alpha^{\prime}$. The aforementioned massive supercharacters are functions of $S O(3)$ fugacity $y$ and $U(1)_{R}$ fugacity $s$.

The fundamental $\mathcal{N}_{4 d}=1$ multiplet ${ }^{17}$ consists of 2 real bosonic degrees of freedom and a Majorana fermion with 2 real fermonic on-shell degrees of freedom after taking the Dirac equation into account, see e.g. [38]. The two real bosonic degrees of freedom can be complexified

14 The floor function $\lfloor\cdot\rfloor$ picks out the nearest integer smaller than or equal to its argument.

15 The lowest $q$ power along with some $S U(2)_{R}$ representation $[n]_{r}$ is generically caused by an operator charged under both $S U(2)_{1}$ and $S U(2)_{2}$. That is why one cannot identify these leading $q$ exponents with the conformal dimension of a simple CFT operator such as an exponential $e^{ \pm i q H}$, see the footnote at the end of Section 3.1.

16 The methods within this work are adapted to the representatives of physical states in the canonical superghost pictures: After stripping off the superghost contributions $e^{q \phi}$ from the $h=1$ vertex operators (with $q=-1$ and $h\left[e^{-\phi}\right]=\frac{1}{2}$ in the NS sector as well as $q=-\frac{1}{2}$ and $h\left[e^{-\phi / 2}\right]=\frac{3}{8}$ in the $\mathrm{R}$ sector), this amounts to counting operators in the matter part of the SCFTs with weight $h=\frac{1}{2}$ in the NS sector and $h=\frac{3}{8}$ in the R sector.

17 As we shall see below, the fundamental multiplet does not appear on its own in both massless and massive spectra. Representations appearing in the massive spectrum arise from certain non-trivial products with the fundamental multiplet. 
to yield a complex scalar and its complex conjugate; they transform as a singlet under the little group $S O(3)$ and each of them carries opposite R-charges +1 and -1 . On the other hand, the two real fermonic degrees of freedom transform as a doublet under the little group $S O(3)$ and each of them carries zero R-charge. Thus, these $2+2$ states yield the character

$$
Z\left(\mathcal{N}_{4 d}=1\right)=[1]_{y}+\left(s+s^{-1}\right)
$$

Any other massive representation of $\mathcal{N}_{4 d}=1$ super-Poincaré is specified by the little group $S O$ (3) quantum number $n$ and the $U(1)_{R}$ charge $Q$ of its highest weight state or Clifford vacuum. Its $S O(3) \times U(1)_{R}$ constituents follow from a tensor product:

$$
\begin{aligned}
\llbracket n, Q \rrbracket & :=Z\left(\mathcal{N}_{4 d}=1\right) \cdot s{ }_{[n]_{y}=s} Q_{[n]_{y}\left([1]_{y}+\left(s+s^{-1}\right)\right)} \\
& =\left\{\begin{array}{cc}
s Q_{\left([n+1]+\left(s+s^{-1}\right)[n]+[n-1]\right)} & \text { for } n \geqslant 1, \\
s Q_{\left([1]+\left(s+s^{-1}\right)[0]\right)} & \text { for } n=0 .
\end{array}\right.
\end{aligned}
$$

The super-Poincaré character $\llbracket n, Q \rrbracket$ corresponds to $4(n+1)$ states of $\operatorname{spin} \frac{n+1}{2}, \frac{n}{2}$ and (if $n \neq 0$ ) $\frac{n-1}{2}$ that can be generated from a Clifford vacuum with spin $n / 2$ and $U(1)_{R}$ charge $Q+1 .{ }^{18}$ Note that $Q$ is even whenever the maximum spin quantum number $n+1$ is.

In this setting, we find the (GSO projected) $\mathcal{N}_{4 d}=1$ partition function

$$
\chi^{\mathcal{N}_{4 d}=1}(q ; y, s):=\left.\chi_{\mathrm{NS}}^{\mathcal{N}_{4 d}=1}\right|_{\mathrm{GSO}}(q ; y, s)+\left.\chi_{\mathrm{R}}^{\mathcal{N}_{4 d}=1}\right|_{\mathrm{GSO}}(q ; y, s),
$$

where GSO projection removes half odd integer mass levels $\alpha^{\prime} m^{2} \in \mathbb{Z}-\frac{1}{2}$ from the NS sector and interlocks spacetime chirality with $U(1)_{R}$ charges in the R sector. We can capture this projection through $^{19}$ :

$$
\begin{aligned}
& \left.\chi_{\mathrm{NS}}^{\mathcal{N}_{4 d}=1}\right|_{\mathrm{GSO}}(q) \\
& \quad=\frac{1}{2} q^{-\frac{1}{2}}\left[\chi_{\mathrm{NS}}^{S O(3)}(q ; y) \chi_{\mathrm{NS}, h=0, \ell=0}^{\mathcal{N}_{2 d}=2, c=9}(q ; s)-\chi_{\mathrm{NS}}^{S O(3)}\left(e^{2 \pi i} q ; y\right) \chi_{\mathrm{NS}, h=0, \ell=0}^{\mathcal{N}_{2 d}=2, c=9}\left(e^{2 \pi i} q ; s\right)\right], \\
& \left.\chi_{\mathrm{R}}^{\mathcal{N}_{4 d}=1}\right|_{\mathrm{GSO}}(q)=\frac{1}{2} \chi_{\mathrm{R}}^{S O(3)}(q ; y) \chi_{\mathrm{R}, h=3 / 8, \ell=3 / 2}^{\mathcal{N}_{2 d}=2, c=9}(q ; s) .
\end{aligned}
$$

In order to compactly represent the leading terms in a power series expansion of the partition function $\chi^{\mathcal{N}_{4 d}=1}$, let us introduce the shorthand

$$
\llbracket n, \pm Q \rrbracket:= \begin{cases}\llbracket n,+Q \rrbracket+\llbracket n,-Q \rrbracket, & Q \neq 0, \\ \llbracket n, 0 \rrbracket, & Q=0\end{cases}
$$

which exploits that $U(1)_{R}$ charges $\pm Q$ always appear on symmetric footing. The pairing of supermultiplets with opposite (non-zero) $U(1)_{R}$ charges combines Majorana fermions as they

\footnotetext{
$\overline{18}$ In this terminology, the first label of $\llbracket n, Q \rrbracket$ refers to the average spin of the $S O(3)$ irreducibles. We deviate from the common practice that supermultiplets are referred to through the highest spin therein. The supercharacter $\llbracket 3,0 \rrbracket=$ [4] $+[2]+\left(s+s^{-1}\right)[3]$, for instance, describes $U(1)_{R}$ neutral bosons of spin two and one, and two massive gravitinos of opposite $U(1)_{R}$ charges.

19 The formula for the GSO projected R sector is reliable for positive powers $q \geqslant 1$ only and inaccurate at the massless level: The coefficient of $q^{0}$ in $\left.\chi_{\mathrm{R}}^{\mathcal{N}_{4 d}=1}\right|_{\text {GSO }}$ is $\frac{1}{2}\left(y+y^{-1}\right)\left(s+s^{-1}\right)$ instead of the desired value $y s+(y s)^{-1}$. One can just add to the former $\frac{1}{2}\left(y-y^{-1}\right)\left(s-s^{-1}\right)$ to compensate this mismatch. This artifact of the mismatch between massive and massless little groups does not affect the main focus our analysis - the massive particle content. Indeed, the character $y s$ corresponds to the left-handed gaugino and the character $(y s)^{-1}$ corresponds to the right-handed gaugino; they carry opposite R-charge +1 and -1 and opposite helicities $+1 / 2$ and $-1 / 2$.
} 
Table 2

The content of the first eight $\mathcal{N}_{4 d}=1$ levels.

\begin{tabular}{|c|c|}
\hline$\alpha^{\prime} m^{2}$ & Representations of $\mathcal{N}_{4 d}=1$ super-Poincaré \\
\hline 1 & $\llbracket 3,0 \rrbracket+\llbracket 0, \pm 1 \rrbracket$ \\
\hline 2 & $\llbracket 5,0 \rrbracket+\llbracket 3,0 \rrbracket+2 \llbracket 2, \pm 1 \rrbracket+2 \llbracket 1,0 \rrbracket$ \\
\hline 3 & $\llbracket 7,0 \rrbracket+\llbracket 5,0 \rrbracket+3 \llbracket 4, \pm 1 \rrbracket+5 \llbracket 3,0 \rrbracket+2 \llbracket 2, \pm 1 \rrbracket+\llbracket 1, \pm 2 \rrbracket+5 \llbracket 1,0 \rrbracket+3 \llbracket 0, \pm 1 \rrbracket$ \\
\hline 4 & $\begin{array}{l}\llbracket 9,0 \rrbracket+\llbracket 7,0 \rrbracket+3 \llbracket 6, \pm 1 \rrbracket+7 \llbracket 5,0 \rrbracket+4 \llbracket 4, \pm 1 \rrbracket+2 \llbracket 3, \pm 2 \rrbracket \\
\quad+12 \llbracket 3,0 \rrbracket+11 \llbracket 2, \pm 1 \rrbracket+2 \llbracket 1, \pm 2 \rrbracket+12 \llbracket 1,0 \rrbracket+3 \llbracket 0, \pm 1 \rrbracket\end{array}$ \\
\hline 5 & $\begin{array}{l}\llbracket 11,0 \rrbracket+\llbracket 9,0 \rrbracket+3 \llbracket 8, \pm 1 \rrbracket+7 \llbracket 7,0 \rrbracket+5 \llbracket 6, \pm 1 \rrbracket+2 \llbracket 5, \pm 2 \rrbracket+17 \llbracket 5,0 \rrbracket+18 \llbracket 4, \pm 1 \rrbracket+6 \llbracket 3, \pm 2 \rrbracket \\
\quad+31 \llbracket 3,0 \rrbracket+20 \llbracket 2, \pm 1 \rrbracket+6 \llbracket 1, \pm 2 \rrbracket+28 \llbracket 1,0 \rrbracket+\llbracket 0, \pm 3 \rrbracket+15 \llbracket 0, \pm 1 \rrbracket\end{array}$ \\
\hline 6 & $\begin{array}{l}\llbracket 13,0 \rrbracket+\llbracket 11,0 \rrbracket+3 \llbracket 10, \pm 1 \rrbracket+7 \llbracket 9,0 \rrbracket+5 \llbracket 8, \pm 1 \rrbracket+2 \llbracket 7, \pm 2 \rrbracket+19 \llbracket 7,0 \rrbracket+21 \llbracket 6, \pm 1 \rrbracket+8 \llbracket 5, \pm 2 \rrbracket \\
\quad+45 \llbracket 5,0 \rrbracket+39 \llbracket 4, \pm 1 \rrbracket+15 \llbracket 3, \pm 2 \rrbracket+72 \llbracket 3,0 \rrbracket+3 \llbracket 2, \pm 3 \rrbracket+58 \llbracket 2, \pm 1 \rrbracket+17 \llbracket 1, \pm 2 \rrbracket+64 \llbracket 1,0 \rrbracket \\
\quad+21 \llbracket 0, \pm 1 \rrbracket\end{array}$ \\
\hline 7 & $\begin{array}{l}\llbracket 15,0 \rrbracket+\llbracket 13,0 \rrbracket+3 \llbracket 12,1 \rrbracket+7 \llbracket 11,0 \rrbracket+5 \llbracket 10,1 \rrbracket+2 \llbracket 9,2 \rrbracket+19 \llbracket 9,0 \rrbracket+22 \llbracket 8,1 \rrbracket+8 \llbracket 7,2 \rrbracket \\
\quad+51 \llbracket 7,0 \rrbracket+49 \llbracket 6,1 \rrbracket+22 \llbracket 5,2 \rrbracket+108 \llbracket 5,0 \rrbracket+4 \llbracket 4,3 \rrbracket+105 \llbracket 4,1 \rrbracket+43 \llbracket 3,2 \rrbracket+166 \llbracket 3,0 \rrbracket \\
\quad+5 \llbracket 2,3 \rrbracket+115 \llbracket 2,1 \rrbracket+38 \llbracket 1,2 \rrbracket+136 \llbracket 1,0 \rrbracket+6 \llbracket 0,3 \rrbracket+66 \llbracket 0,1 \rrbracket\end{array}$ \\
\hline 8 & $\begin{array}{l}\llbracket 17,0 \rrbracket+\llbracket 15,0 \rrbracket+3 \llbracket 14,1 \rrbracket+7 \llbracket 13,0 \rrbracket+5 \llbracket 12,1 \rrbracket+2 \llbracket 11,2 \rrbracket+19 \llbracket 11,0 \rrbracket+22 \llbracket 10,1 \rrbracket+8 \llbracket 9,2 \rrbracket \\
\quad+53 \llbracket 9,0 \rrbracket+52 \llbracket 8,1 \rrbracket+24 \llbracket 7,2 \rrbracket+125 \llbracket 7,0 \rrbracket+4 \llbracket 6,3 \rrbracket+135 \llbracket 6,1 \rrbracket+62 \llbracket 5,2 \rrbracket+254 \llbracket 5,0 \rrbracket \\
\quad+10 \llbracket 4,3 \rrbracket+223 \llbracket 4,1 \rrbracket+101 \llbracket 3,2 \rrbracket+357 \llbracket 3,0 \rrbracket+21 \llbracket 2,3 \rrbracket+274 \llbracket 2,1 \rrbracket+\llbracket 1,4 \rrbracket+89 \llbracket 1,2 \rrbracket \\
\quad+289 \llbracket 1,0 \rrbracket+7 \llbracket 0,3 \rrbracket+112 \llbracket 0,1 \rrbracket\end{array}$ \\
\hline
\end{tabular}

appear in the fundamental multiplet (4.1) to Dirac fermions. The content of the first $\mathcal{N}_{4 d}=1$ levels reads

$$
\begin{aligned}
\chi^{\mathcal{N}_{4 d}=1}(q ; y, s)= & \underbrace{\left(y^{2}+y^{-2}+\frac{1}{2}\left(y+y^{-1}\right)\left(s+s^{-1}\right)\right) q^{0}}_{4 \text { massless states }}+\underbrace{(\llbracket 3,0 \rrbracket+\llbracket 0, \pm 1 \rrbracket) q}_{24 \text { states at level } 1} \\
& +\underbrace{(\llbracket 5,0 \rrbracket+\llbracket 3,0 \rrbracket+2 \llbracket 2, \pm 1 \rrbracket+2 \llbracket 1,0 \rrbracket) q^{2}}_{104 \text { states at level } 2} \\
& +(\llbracket 7,0 \rrbracket+\llbracket 5,0 \rrbracket+3 \llbracket 4, \pm 1 \rrbracket+5 \llbracket 3,0 \rrbracket+2 \llbracket 2, \pm 1 \rrbracket \\
& +\llbracket 1, \pm 2 \rrbracket+5 \llbracket 1,0 \rrbracket+3 \llbracket 0, \pm 1 \rrbracket) q^{3}+\mathcal{O}\left(q^{4}\right),
\end{aligned}
$$

subleading orders up to mass level eight are summarized in Table 2. The explicit form of the vertex operators at mass level one ${ }^{20}$ can be found in Section 5 of [33] (Eqs. (5.3) to (5.6) for bosons and Eqs. (5.14) to (5.18) for fermions) in the RNS framework, and Refs. [39,40] provide their superspace description.

Character multiplicities up to mass level $\alpha^{\prime} m^{2}=25$ are gathered in Table 3 and in the tables of Appendix B.1.

20 Let us discuss about the states at the first mass level. The 24 total states consist of the following multiplets:

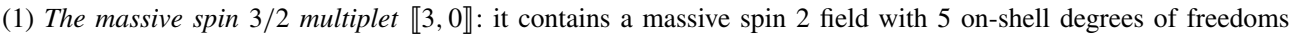
(OSDOFs), a massive spin 1 field with 3 OSDOFs, a massive spin 3/2 field with 4 OSDOFs, and a Dirac fermion with 4 OSDOFs; so we have $8+8$ real OSDOFs in total

(2) The massive spin 0 multiplet $\llbracket 0, \pm 1 \rrbracket$ : the two constituents $\llbracket 0,1 \rrbracket$ and $\llbracket 0,-1 \rrbracket$ of the massive scalar multiplet correspond to two massless chiral fields, $\Phi$ and $\widetilde{\Phi}$ (not complex conjugate to each other) at $Q= \pm 1$. The opposite $Q$-charges are necessary to form an invariant mass term $\Phi \widetilde{\Phi}$ in the superpotential. This multiplet contains $4+4$ real OSDOFs coming from two complex scalars plus two Majorana fermions; the latter are equivalent to one massive Dirac fermion. Note that the spin 0 multiplet is also referred to as two spin $1 / 2$ multiplets in [33]. 


\subsection{The total number of states at a given mass level}

In this subsection, we focus on the total number of states present at a given mass level and derive the novel asymptotic formula (4.17). These numbers can indeed be obtained by adding up the dimensions of representations presented in Table 2. Our aim here is to compute such numbers analytically and asymptotically for large mass levels.

The starting point is the unrefined partition function obtained by setting the fugacities $y$ and $s$ in (4.3) to unity. The total number of states $N_{m}$ at the mass level $m$ can be read off from the coefficient of $q^{m}$ in the power series of $\chi^{\mathcal{N}_{4 d}=1}(q ; y=1, s=1)$.

Supersymmetry implies that

$$
\left.\chi_{\mathrm{NS}}^{\mathcal{N}_{4 d}=1}\right|_{\mathrm{GSO}}(q ; y=1, s=1)=\left.\chi_{\mathrm{R}}^{\mathcal{N}_{4 d}=1}\right|_{\mathrm{GSO}}(q ; y=1, s=1) .
$$

which can, of course, be checked directly using (4.4), (2.27), (2.44), (3.3) and (3.5). Since the formula for the $\mathrm{R}$ sector is simpler, we proceed from there.

$$
\begin{aligned}
\chi^{\mathcal{N}_{4 d}=1}(q ; y=1, s=1) & =\left.2 \chi_{\mathrm{R}}^{\mathcal{N}_{4 d}=1}\right|_{\mathrm{GSO}}(q ; y=1, s=1) \\
& =\chi_{\mathrm{R}}^{S O(3)}(q, y=1) \chi_{\mathrm{R}, h=3 / 8, \ell=3 / 2}^{\mathcal{N}_{2 d}=2, c=9}(q ; s=1) \\
& =q^{-1 / 4} \frac{\vartheta_{2}(1, q)^{2}}{\eta(q)^{6}}\left[\vartheta_{2}\left(1, q^{2}\right)-q^{1 / 4} \vartheta_{3}\left(1, q^{2}\right)\right] .
\end{aligned}
$$

Indeed, the power series of $\chi^{\mathcal{N}_{4 d}=1}(q ; y=1, s=1)$ in $q$ reproduces the numbers presented in the first column of Table 1. We mention in passing that $\chi^{\mathcal{N}_{4 d}=1}(q ; y=1, s=1)$ is not a modular form.

\subsubsection{The number of states at each mass level and its asymptotics}

The number of states at the mass level $m$ can be computed from

$$
N_{m}=\frac{1}{2 \pi i} \oint_{\mathcal{C}} \frac{\mathrm{d} q}{q^{m+1}} \chi^{\mathcal{N}_{4 d}=1}(q ; y=1, s=1),
$$

where $\mathcal{C}$ is a contour around the origin.

Let us compute the number of states $N_{m}$ in the limit $m \rightarrow \infty$. Since the integrand of (4.9) is sharply peaked near $q=1$, we need to examine the behavior of $\chi^{\mathcal{N}_{4 d}=1}(q ; y=1, s=1)$ as $q \rightarrow 1^{-}$. The $q \rightarrow 1^{-}$regime in question is related to the easily accessible $q \rightarrow 0$ limit

$$
\eta(q) \sim q^{1 / 24}, \quad \vartheta_{3}(1, q) \sim 1, \quad \vartheta_{4}(1, q) \sim 1, \quad q \rightarrow 0
$$

through modular transformation $q=e^{2 \pi i \tau} \mapsto \widetilde{q}=e^{-2 \pi i / \tau}$ :

$$
\begin{aligned}
& \vartheta_{2} \text {-function: } \quad \vartheta_{4}(1, \tilde{q})=\vartheta_{2}(1, q) \sqrt{-i \tau} \sim \frac{1}{\sqrt{2 \pi}}(1-q)^{1 / 2} \vartheta_{2}(1, q) \\
& \Rightarrow \quad \vartheta_{2}(1, q) \sim \sqrt{2 \pi}(1-q)^{-1 / 2}, \quad q \rightarrow 1^{-}, \\
& \vartheta_{3} \text {-function: } \quad \vartheta_{3}(1, \tilde{q})=\vartheta_{3}(1, q) \sqrt{-i \tau} \sim \frac{1}{\sqrt{2 \pi}}(1-q)^{1 / 2} \vartheta_{3}(1, q) \\
& \Rightarrow \quad \vartheta_{3}(1, q) \sim \sqrt{2 \pi}(1-q)^{-1 / 2}, \quad q \rightarrow 1^{-},
\end{aligned}
$$




$$
\begin{aligned}
\eta \text {-function: } \quad \eta(\tilde{q})=\eta(q) \sqrt{-i \tau} \sim \frac{1}{\sqrt{2 \pi}}(1-q)^{1 / 2} \eta(q) \\
\Rightarrow \quad \eta(q) \sim \sqrt{2 \pi}(1-q)^{-1 / 2} \exp \left(\frac{\pi^{2}}{6 \log q}\right), \quad q \rightarrow 1^{-} .
\end{aligned}
$$

Hence, we have

$$
\vartheta_{2}\left(1, q^{2}\right) \sim \vartheta_{3}\left(1, q^{2}\right) \sim \sqrt{2 \pi}\left(1-q^{2}\right)^{-1 / 2}, \quad q \rightarrow 1^{-},
$$

and so as $q \rightarrow 1^{-}$,

$$
\begin{aligned}
& \chi^{\mathcal{N}_{4 d}=1}(q ; y=1, s=1) \\
& \quad \sim(2 \pi)^{-3 / 2}(1-q)^{2}\left(1-q^{1 / 4}\right)\left(1-q^{2}\right)^{-1 / 2} \exp \left(-\frac{\pi^{2}}{\log q}\right) .
\end{aligned}
$$

Hence, as $m \rightarrow \infty$,

$$
\begin{aligned}
N_{m} \sim & (2 \pi)^{-3 / 2} \frac{1}{2 \pi i} \oint_{\mathcal{C}} \frac{\mathrm{d} q}{q}(1-q)^{2}\left(1-q^{1 / 4}\right)\left(1-q^{2}\right)^{-1 / 2} \\
& \times \exp \left(-\frac{\pi^{2}}{\log q}-m \log q\right) .
\end{aligned}
$$

Observe that the argument of the exponential function has a critical value at $q_{0}=$ $\exp (-\pi / \sqrt{m})$; this is the saddle point. The direction of steepest descent at this point is the imaginary direction in $q$. We deform the contour $\mathcal{C}$ such that it passes through $q=q_{0}$ and tangent to this direction. The leading contribution comes from expansions around $q=q_{0}$ in the steepest descent direction. Writing $q=q_{0} e^{i \theta}$, we have

$$
\begin{aligned}
N_{m} \sim & (2 \pi)^{-3 / 2}\left(1-q_{0}\right)^{2}\left(1-q_{0}^{1 / 4}\right)\left(1-q_{0}^{2}\right)^{-1 / 2} \\
& \times \frac{1}{2 \pi} \int_{-\epsilon}^{\epsilon} \mathrm{d} \theta \exp \left(-\frac{\pi^{2}}{i \theta+\log q_{0}}-m\left(i \theta+\log q_{0}\right)\right), \quad \epsilon>0 \\
\sim & (2 \pi)^{-3 / 2}\left(1-q_{0}\right)^{2}\left(1-q_{0}^{1 / 4}\right)\left(1-q_{0}^{2}\right)^{-1 / 2} \\
& \times e^{2 \pi \sqrt{m}} \frac{1}{2 \pi} \int_{-\epsilon}^{\epsilon} \mathrm{d} \theta \exp \left(-\frac{m^{3 / 2}}{\pi} \theta^{2}+O\left(\theta^{3}\right)\right), \quad \epsilon>0 \\
\sim & (2 \pi)^{-3 / 2}\left(1-q_{0}\right)^{2}\left(1-q_{0}^{1 / 4}\right)\left(1-q_{0}^{2}\right)^{-1 / 2} e^{2 \pi \sqrt{m}} \frac{1}{2 \pi} \int_{-\infty}^{\infty} \mathrm{d} \theta \exp \left(-\frac{m^{3 / 2}}{\pi} \theta^{2}\right) \\
\sim & \frac{\pi}{32} m^{-2} \exp (2 \pi \sqrt{m}), \quad m \rightarrow \infty .
\end{aligned}
$$

\subsection{The GSO projected NS and R sectors}

In what follows, we compute analytic expressions of the refined partition function $\chi^{\mathcal{N}_{4 d}=1}(q ; y, s)$ and discuss its asymptotic behavior. 


\subsubsection{The NS sector}

Let us write the partition function $\left.\chi_{\mathrm{NS}}^{\mathcal{N}_{4 d}=1}\right|_{\mathrm{GSO}}(q ; y, s)$, defined in (4.4), as

$$
\left.\chi_{\mathrm{NS}}^{\mathcal{N}_{4 d}=1}\right|_{\mathrm{GSO}}(q ; y, s)=\sum_{k=0}^{\infty} \sum_{p=-\infty}^{\infty}[2 k]_{y} s^{2 p} F_{k, p}^{\mathrm{NS}}(q),
$$

where the function $F_{k, p}^{\mathrm{NS}}(q)$ follows from (2.25), (3.1) and (4.4):

$$
\begin{aligned}
F_{k, p}^{\mathrm{NS}}(q)= & (q ; q)_{\infty}^{-6}(1-q) q^{p^{2}+p-1} \sum_{n=1}^{\infty}(-1)^{n+1}\left(1-q^{n}\right) q^{\left(\begin{array}{c}
n \\
2
\end{array}\right)} \sum_{m=0}^{\infty}\left(q^{n|k-m|}-q^{n(k+m+1)}\right) \\
& \times \frac{1}{2} q^{\frac{1}{2} m^{2}}\left[\frac{\left(1-q^{m+\frac{1}{2}}\right) \vartheta_{3}(1, q)}{\left(1+q^{p-\frac{1}{2}}\right)\left(1+q^{p+\frac{1}{2}}\right)}+(-1)^{m^{2}} \frac{\left(1+q^{m+\frac{1}{2}}\right) \vartheta_{4}(1, q)}{\left(1-q^{p-\frac{1}{2}}\right)\left(1-q^{p+\frac{1}{2}}\right)}\right] .
\end{aligned}
$$

This expression can be simplified further in the asymptotic limit $k \rightarrow \infty$. In this limit, $q^{n|k-m|} \sim q^{n(k-m)}$ and the dominant contribution in the summation over $n$ comes from $n=1$. The summation over $n$ can be asymptotically evaluated as follows (assume that $m$ is finite):

$$
\begin{gathered}
\sum_{n=1}^{\infty}(-1)^{n+1}\left(1-q^{n}\right) q^{\left(\begin{array}{c}
n \\
2
\end{array}\right)}\left(q^{n|k-m|}-q^{n(k+m+1)}\right) \\
\sim \sum_{n=1}^{\infty}(-1)^{n+1}\left(1-q^{n}\right) q^{n(k-m)}\left(1-q^{n(2 m+1)}\right) \\
\sim \frac{q^{k}(1-q)\left(1-q^{2 k}\right)}{\left(1+q^{k}\right)^{4}}\left\{q^{-m}\left(1-q^{2 m+1}\right)\right\} .
\end{gathered}
$$

The summation over $m$ can be evaluated by considering

$$
\begin{aligned}
& \sum_{m=0}^{\infty} q^{\frac{1}{2} m^{2}-m}\left(1-q^{m+\frac{1}{2}}\right)\left(1-q^{2 m+1}\right)=q^{-\frac{1}{2}}(1-q) \vartheta_{3}(1, q) \\
& \sum_{m=0}^{\infty}(-1)^{m^{2}} q^{\frac{1}{2} m^{2}-m}\left(1+q^{m+\frac{1}{2}}\right)\left(1-q^{2 m+1}\right)=-q^{-\frac{1}{2}}(1-q) \vartheta_{4}(1, q)
\end{aligned}
$$

In such a limit, the function $F_{k, p}^{\mathrm{NS}}(q)$ becomes

$$
\begin{aligned}
F_{k, p}^{\mathrm{NS}}(q) \sim & \frac{1}{2}(q ; q)_{\infty}^{-6}(1-q)^{3} q^{p^{2}+p+k-\frac{3}{2}} \frac{1-q^{2 k}}{\left(1+q^{k}\right)^{4}} \\
& \times\left[\frac{\vartheta_{3}(1, q)^{2}}{\left(1+q^{p-\frac{1}{2}}\right)\left(1+q^{p+\frac{1}{2}}\right)}-\frac{\vartheta_{4}(1, q)^{2}}{\left(1-q^{p-\frac{1}{2}}\right)\left(1-q^{p+\frac{1}{2}}\right)}\right] \\
\sim & \frac{1}{2}(q ; q)_{\infty}^{-6}(1-q)^{3} q^{p^{2}+p+k-\frac{3}{2}} \\
& \times\left[\frac{\vartheta_{3}(1, q)^{2}}{\left(1+q^{p-\frac{1}{2}}\right)\left(1+q^{p+\frac{1}{2}}\right)}-\frac{\vartheta_{4}(1, q)^{2}}{\left(1-q^{p-\frac{1}{2}}\right)\left(1-q^{p+\frac{1}{2}}\right)}\right], \quad k \rightarrow \infty
\end{aligned}
$$




\subsubsection{The $R$ sector}

Similarly the partition function $\left.\chi_{\mathrm{R}}^{\mathcal{N}_{4 d}=1}\right|_{\mathrm{GSO}}(q ; y, s)$, defined in (4.4), can be written as

$$
\left.\chi_{\mathrm{R}}^{\mathcal{N}_{4 d}=1}\right|_{\mathrm{GSO}}(q ; y, s)=\sum_{k=0}^{\infty} \sum_{p=-\infty}^{\infty}[2 k+1]_{y} s^{2 p-1} F_{k, p}^{\mathrm{R}}(q),
$$

where the function $F_{k, p}^{\mathrm{R}}(q)$ follows from (2.42), (3.4) and (4.4):

$$
\begin{aligned}
F_{k, p}^{\mathrm{R}}(q)= & \frac{1}{2}(q ; q)_{\infty}^{-6}(1-q) \frac{q^{p^{2}-\frac{5}{4}}}{\left(1+q^{p}\right)\left(1+q^{p-1}\right)} \vartheta_{2}(1, q) \\
& \times \sum_{n=1}^{\infty}(-1)^{n+1}\left(1-q^{n}\right) q^{\left(\begin{array}{l}
n \\
2
\end{array}\right)} \sum_{m=0}^{\infty} q^{\frac{1}{2}\left(m+\frac{1}{2}\right)^{2}}\left(1-q^{m+1}\right)\left(q^{n|k-m|}-q^{n(k+m+2)}\right) .
\end{aligned}
$$

In the limit $k \rightarrow \infty$, this function can be simplified further. The summation over $n$ can be asymptotically evaluated as follows (assume that $m$ is finite):

$$
\begin{gathered}
\sum_{n=1}^{\infty}(-1)^{n+1}\left(1-q^{n}\right) q^{\left(\begin{array}{l}
n \\
2
\end{array}\right)}\left(q^{n|k-m|}-q^{n(k+m+2)}\right) \\
\sim \sum_{n=1}^{\infty}(-1)^{n+1}\left(1-q^{n}\right) q^{n(k-m)}\left(1-q^{n(2 m+2)}\right) \\
\sim \frac{q^{k}(1-q)\left(1-q^{2 k}\right)}{\left(1+q^{k}\right)^{4}}\left\{q^{-m}\left(1-q^{2 m+2}\right)\right\},
\end{gathered}
$$

and the summation over $m$ can be computed as follows:

$$
\sum_{m=0}^{\infty} q^{\frac{1}{2}\left(m+\frac{1}{2}\right)^{2}-m}\left(1-q^{m+1}\right)\left(1-q^{2 m+2}\right)=(1-q) \vartheta_{2}(1, q) .
$$

Therefore, we have the following asymptotic formula:

$$
\begin{aligned}
F_{k, p}^{\mathrm{R}}(q) & \sim \frac{1}{2}(q ; q)_{\infty}^{-6} \frac{q^{p^{2}+k-\frac{5}{4}}(1-q)^{3}\left(1-q^{2 k}\right)}{\left(1+q^{p}\right)\left(1+q^{p-1}\right)\left(1+q^{k}\right)^{4}} \vartheta_{2}(1, q)^{2} \\
& \sim \frac{1}{2}(q ; q)_{\infty}^{-6} \frac{q^{p^{2}+k-\frac{5}{4}}(1-q)^{3}}{\left(1+q^{p}\right)\left(1+q^{p-1}\right)} \vartheta_{2}(1, q)^{2}, \quad k \rightarrow \infty
\end{aligned}
$$

\subsubsection{Combining both sectors}

Combining the NS and R contributions from the previous subsections gives rise to the following $S O(3) \times U(1)_{R}$ covariant partition function

$$
\begin{aligned}
\chi^{\mathcal{N}_{4 d}=1}(q ; y, s) & =\sum_{k=0}^{\infty} \sum_{p=-\infty}^{\infty}\left([2 k]_{y} s^{2 p} F_{k, p}^{\mathrm{NS}}(q)+[2 k+1]_{y} s^{2 p-1} F_{k, p}^{\mathrm{R}}(q)\right) \\
& =\sum_{k=0}^{\infty}\left\{[2 k]\left(F_{k, 0}^{\mathrm{NS}}(q)+\sum_{p=1}^{\infty} s_{2 p} F_{k, p}^{\mathrm{NS}}(q)\right)+[2 k+1] \sum_{p=1}^{\infty} s_{2 p-1} F_{k, p}^{\mathrm{R}}(q)\right\},
\end{aligned}
$$


where $s_{m}$ is defined by (3.2). Even though the $F_{k, p}^{\mathrm{NS}}$ and $F_{k, p}^{\mathrm{R}}$ functions are known, the representation (4.29) of the overall partition function does not make $\mathcal{N}_{4 d}=1$ SUSY manifest to all mass levels. In order to do so, we have to combine $S O(3) \times U(1)_{R}$ representations to supermultiplets (4.2) and rewrite (4.29) as $^{21}$

$$
\chi^{\mathcal{N}_{4 d}=1}(q ; y, s)=\sum_{n=0}^{\infty} \sum_{Q=0}^{\infty} \llbracket n, \pm Q \rrbracket M\left(\chi^{\mathcal{N}_{4 d}=1}, \llbracket n, Q \rrbracket, q\right) .
$$

This introduces a multiplicity generating function $M\left(\chi^{\mathcal{N}_{4 d}=1}, \llbracket n, Q \rrbracket, q\right)$ for the supermultiplet $\llbracket n, Q \rrbracket$ appearing in the partition function $\chi^{\mathcal{N}_{4 d}=1}$. To lighten our notation in the subsequent steps, we shall use the shorthand

$$
G_{n, Q}(q):=M\left(\chi^{\mathcal{N}_{4 d}=1}, \llbracket n, Q \rrbracket, q\right) .
$$

By comparing (4.29) with (4.30), it is immediate that

$$
G_{2 n, 2 Q}(q)=G_{2 n+1,2 Q+1}(q)=0, \quad \text { for all } n \geqslant 0 \text { and } Q \geqslant 0 .
$$

\subsubsection{Recurrence relations}

In order to relate the supersymmetric multiplicity generating functions $G_{n, Q}$ to their $S O(3) \times$ $U(1)_{R}$ relatives $F_{k, p}^{\mathrm{NS}}$ and $F_{k, p}^{\mathrm{R}}$, we use (4.2) to rewrite (4.30) in terms of characters of irreducible $S O(3)$ characters and the fugacity $s$ as

$$
\begin{aligned}
\chi^{\mathcal{N}_{4 d}=1}(q ; y, s)= & {[0]\left[\left(G_{1,0}+2 G_{0,1}\right)+\sum_{Q=1}^{\infty} s_{2 Q}\left(G_{0,2 Q-1}+G_{1,2 Q}+G_{0,2 Q+1}\right)\right] } \\
& +\sum_{k=1}^{\infty}[2 k]\left[\left(G_{2 k-1,0}+2 G_{2 k, 1}+G_{2 k+1,0}\right)\right. \\
& \left.+\sum_{Q=1}^{\infty} s_{2 Q}\left(G_{2 k-1,2 Q}+G_{2 k, 2 Q-1}+G_{2 k, 2 Q+1}+G_{2 k+1,2 Q}\right)\right] \\
& +\sum_{k=0}^{\infty}[2 k+1] \sum_{Q=1}^{\infty} s_{2 Q-1}\left(G_{2 k, 2 Q-1}+G_{2 k+1,2 Q-2}\right. \\
& \left.+G_{2 k+1,2 Q}+G_{2 k+2,2 Q-1}\right),
\end{aligned}
$$

where $G_{n, Q}$ is a shorthand notation for $G_{n, Q}(q)$.

Comparing (4.29) with (4.33), we have the following relations:

$$
\begin{aligned}
& 2 G_{0,1}(q)+G_{1,0}(q)=F_{0,0}^{\mathrm{NS}}(q), \\
& G_{2 k-1,0}(q)+2 G_{2 k, 1}(q)+G_{2 k+1,0}(q)=F_{k, 0}^{\mathrm{NS}}(q), \quad k \geqslant 1, \\
& G_{0,2 Q-1}(q)+G_{0,2 Q+1}(q)+G_{1,2 Q}(q)=F_{0, Q}^{\mathrm{NS}}(q), \quad Q \geqslant 1, \\
& G_{2 k-1,2 Q}(q)+G_{2 k, 2 Q-1}(q)+G_{2 k, 2 Q+1}(q)+G_{2 k+1,2 Q}(q)=F_{k, Q}^{\mathrm{NS}}(q), \\
& \quad k, Q \geqslant 1,
\end{aligned}
$$

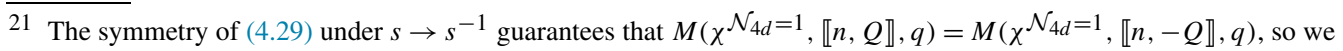
shall henceforth assume that $Q \geqslant 0$. 


$$
\begin{aligned}
& G_{2 k, 2 Q-1}(q)+G_{2 k+1,2 Q-2}(q)+G_{2 k+1,2 Q}(q)+G_{2 k+2,2 Q-1}(q)=F_{k, Q}^{\mathrm{R}}(q), \\
& \quad k \geqslant 0, \quad Q \geqslant 1 .
\end{aligned}
$$

These relations are useful for computing a multiplicity generating function for a representation

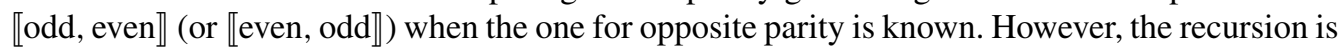
not powerful enough to directly determine all the $G_{n, Q}$ in terms of $F_{k, p}^{\mathrm{NS}}$ and $F_{k, p}^{\mathrm{R}}$. The following subsection follows an alternative approach to determine the $G_{n, Q}$.

\subsection{Multiplicities of representations in the $\mathcal{N}_{4 d}=1$ partition function}

Our aim in this subsection is to factor out the fundamental $\mathcal{N}_{4 d}=1$ super-Poincare character $Z\left(\mathcal{N}_{4 d}=1\right)=[1]_{y}+s+s^{-1}$ and to compute explicitly the multiplicity generating functions $G_{n, Q}(q)$ for $\llbracket n, Q \rrbracket$ in

$$
\chi^{\mathcal{N}_{4 d}=1}(q ; y, s)=\sum_{n=0}^{\infty} \sum_{Q=-\infty}^{\infty} \llbracket n, Q \rrbracket G_{n, Q}(q) .
$$

Using the second equality of (4.2) and orthogonality of $S O(3) \times U(1)_{R}$ representations, we have

$$
\begin{aligned}
G_{n, Q}(q) & =M\left(\chi^{\mathcal{N}_{4 d}=1}, \llbracket n, Q \rrbracket, q\right) \\
& =\frac{1}{2 \pi i} \oint_{\mathcal{C}} \frac{\mathrm{d} s}{s} \int \mathrm{d} \mu_{S O(3)}(y)[n]_{y} s-Q \frac{\chi^{\mathcal{N}_{4 d}=1}(q ; y, s)}{[1]_{y}+\left(s+s^{-1}\right)},
\end{aligned}
$$

where $\mathcal{C}$ is a contour in the complex $s$-plane enclosing the origin. In order to proceed, we use the geometric series expansion of the inverse $Z\left(\mathcal{N}_{4 d}=1\right),{ }^{22}$

$$
\frac{1}{[1]_{y}+\left(s+s^{-1}\right)}=\frac{1}{s+s^{-1}} \frac{1}{1+\frac{[1]_{y}}{s+s^{-1}}}=\sum_{m=0}^{\infty}(-1)^{m} \frac{[1]_{y}^{m}}{\left(s+s^{-1}\right)^{m+1}} .
$$

In what follows, we consider the contributions from $\left.\chi_{\mathrm{NS}}^{\mathcal{N}_{4 d}=1}\right|_{\mathrm{GSO}}(q ; y, s)$ and $\left.\chi_{\mathrm{R}}^{\mathcal{N}_{4 d}=1}\right|_{\mathrm{GSO}}(q ; y, s)$ separately and then add up these results to yield the overall multiplicity generating function defined by (4.39),

$$
M\left(\chi^{\mathcal{N}_{4 d}=1}, \llbracket n, Q \rrbracket, q\right)=M\left(\left.\chi_{\mathrm{NS}}^{\mathcal{N}_{4 d}=1}\right|_{\mathrm{GSO}}, \llbracket n, Q \rrbracket, q\right)+M\left(\left.\chi_{\mathrm{R}}^{\mathcal{N}_{4 d}=1}\right|_{\mathrm{GSO}}, \llbracket n, Q \rrbracket, q\right),
$$

where $\left.\chi_{\mathrm{NS}, \mathrm{R}}^{\mathcal{N}_{4 d}=1}\right|_{\text {GSO }}$ are given by (4.18) and (4.24).

$\overline{22}$ Note that $\frac{1}{[1]_{y}+\left(s+s^{-1}\right)}$ can also be written in another way as follows:

$$
\frac{1}{[1]_{y}+\left(s+s^{-1}\right)}=\sum_{m=0}^{\infty}(-1)^{m} s^{m+1}[m]_{y} .
$$

However, we shall not take this approach, since otherwise this would lead to tensor products in (4.46) and (4.47) which are harder to evaluate in comparison with our current approach. 


\subsubsection{Multiplicities in the NS sector}

The series expansion of $\left(Z\left(\mathcal{N}_{4 d}=1\right)\right)^{-1}$ leads to the following NS sector contribution to the multiplicity generating function of the supermultiplet $\llbracket n, Q \rrbracket$

$$
\begin{aligned}
& M\left(\left.\chi_{\mathrm{NS}}^{\mathcal{N}_{4 d}=1}\right|_{\mathrm{GSO}}, \llbracket n, Q \rrbracket, q\right) \\
& :=\frac{1}{2 \pi i} \oint_{\mathcal{C}} \frac{\mathrm{d} s}{s} \int \mathrm{d} \mu_{S O(3)}(y) \frac{[n]_{y}}{s Q} \times \frac{\left.\chi_{\mathrm{NS}}^{\mathcal{N}_{4 d}=1}\right|_{\mathrm{GSO}}(q ; y, s)}{[1]_{y}+\left(s+s^{-1}\right)} \\
& =\sum_{m=0}^{\infty} \sum_{k=0}^{\infty} \sum_{p=-\infty}^{\infty}(-1)^{m} F_{k, p}^{\mathrm{NS}}(q) \frac{1}{2 \pi i} \\
& \times \oint_{|s|=1-\epsilon} \frac{\mathrm{d} s}{s} \frac{s^{2 p}}{s^{Q}\left(s+s^{-1}\right)^{m+1}} \int \mathrm{d} \mu_{S O(3)}(y)[n]_{y}[1]_{y}^{m}[2 k]_{y} .
\end{aligned}
$$

We shall henceforth take $\mathcal{C}$ to be a circle centred at the origin with the radius $1-\epsilon$, with $0<\epsilon<1$. The quantities in the curly brackets can be computed as follows:

$$
\begin{aligned}
& \frac{1}{2 \pi i} \oint_{|s|=1-\epsilon} \frac{\mathrm{d} s}{s} \frac{s^{2 p}}{s^{Q}\left(s+s^{-1}\right)^{m+1}} \\
& = \begin{cases}(-1)^{\frac{1}{2}(Q-m-2 p-1)}\left(\begin{array}{c}
\frac{1}{2}(Q+m-2 p-1) \\
m
\end{array}\right. & \text { for } Q-m \text { odd and } Q+m \geqslant 2 p+1, \\
0 & \text { otherwise, }\end{cases}
\end{aligned}
$$

and

$$
\begin{aligned}
& \int \mathrm{d} \mu_{S O(3)}(y)[2 n]_{y}[1]_{y}^{m}[2 k]_{y}= \begin{cases}T_{2 n+1}\left(m, \frac{1}{2} m+n-k\right) & \text { if } m \text { is even, } \\
0 & \text { if } m \text { is odd },\end{cases} \\
& \int \mathrm{d} \mu_{S O(3)}(y)[2 n+1]_{y}[1]_{y}^{m}[2 k]_{y} \\
& = \begin{cases}T_{2 n+2}\left(m, \frac{1}{2} m+n+\frac{1}{2}-k\right) & \text { if } m \text { is odd, } \\
0 & \text { if } m \text { is even, }\end{cases}
\end{aligned}
$$

where

$$
T_{p}(m, k)=\left(\begin{array}{c}
m \\
k
\end{array}\right)-\left(\begin{array}{c}
m \\
k-p
\end{array}\right)
$$

Note that (4.45), (4.46) and (4.47) are in perfect agreement with the selection rule

$$
M\left(\chi^{\mathcal{N}_{4 d}=1}, \llbracket 2 n, 2 Q \rrbracket, q\right)=M\left(\chi^{\mathcal{N}_{4 d}=1}, \llbracket 2 n+1,2 Q+1 \rrbracket, q\right)=0 .
$$

The non-zero multiplicities of $\llbracket 2 n, 2 Q+1 \rrbracket$ and $\llbracket 2 n+1,2 Q \rrbracket$ receive the following NS sector contributions:

$$
\begin{aligned}
M & \left(\left.\chi_{\mathrm{NS}}^{\mathcal{N}_{4 d}=1}\right|_{\mathrm{GSO}}, \llbracket 2 n, 2 Q+1 \rrbracket, q\right) \\
& =\sum_{k=0}^{\infty} \sum_{m=0}^{\infty} \sum_{p=-\infty}^{Q+m}(-1)^{Q-m-p} F_{k, p}^{\mathrm{NS}}(q)\left(\begin{array}{c}
Q+m-p \\
2 m
\end{array}\right) T_{2 n+1}(2 m, m+n-k),
\end{aligned}
$$




$$
\begin{aligned}
M & \left(\left.\chi_{\mathrm{NS}}^{\mathcal{N}_{4 d}=1}\right|_{\mathrm{GSO}}, \llbracket 2 n+1,2 Q \rrbracket, q\right) \\
& =\sum_{k=0}^{\infty} \sum_{m=0}^{\infty} \sum_{p=-\infty}^{Q+m}(-1)^{Q-m-p} F_{k, p}^{\mathrm{NS}}(q)\left(\begin{array}{c}
Q+m-p \\
2 m+1
\end{array}\right) T_{2 n+2}(2 m+1, m+n+1-k) .
\end{aligned}
$$

\subsubsection{Multiplicities in the $R$ sector}

Similarly to the NS sector, the generating function for the multiplicity of the representation $\llbracket n, Q \rrbracket$ in the function $\left.\chi_{\mathrm{R}}^{\mathcal{N}_{4 d}=1}\right|_{\mathrm{GSO}}(q ; y, s)$ is given by

$$
\begin{aligned}
& M\left(\left.\chi_{\mathrm{R}}^{\mathcal{N}_{4 d}=1}\right|_{\mathrm{GSO}}, \llbracket n, Q \rrbracket, q\right) \\
& :=\frac{1}{2 \pi i} \oint_{|s|=1-\epsilon} \frac{\mathrm{d} s}{s} \int \mathrm{d} \mu_{S O(3)}(y) \frac{[n]_{y}}{s Q} \times \frac{\left.\chi_{\mathrm{R}}^{\mathcal{N}_{4 d}=1}\right|_{\mathrm{GSO}}(q ; y, s)}{[1]_{y}+\left(s+s^{-1}\right)} \\
& =\sum_{m=0}^{\infty} \sum_{k=0}^{\infty} \sum_{p=-\infty}^{\infty}(-1)^{m} F_{k, p}^{\mathrm{R}}(q) \frac{1}{2 \pi i} \\
& \times \oint_{|s|=1-\epsilon} \frac{\mathrm{d} s}{s} \frac{s^{2 p-1}}{s^{Q}\left(s+s^{-1}\right)^{m+1}} \int \mathrm{d} \mu_{S O(3)}(y)[n]_{y}[1]_{y}^{m}[2 k+1]_{y},
\end{aligned}
$$

with $0<\epsilon<1$,

$$
\begin{aligned}
& \frac{1}{2 \pi i} \oint_{|s|=1-\epsilon} \frac{\mathrm{d} s}{s} \frac{s^{2 p-1}}{s^{Q}\left(s+s^{-1}\right)^{m+1}} \\
& = \begin{cases}(-1)^{\frac{1}{2}(Q-m-2 p)}\left(\begin{array}{c}
\frac{1}{2}(Q+m-2 p) \\
m
\end{array}\right) & \text { for } Q-m \text { even and } Q+m \geqslant 2 p, \\
0 & \text { otherwise }\end{cases}
\end{aligned}
$$

and

$$
\begin{aligned}
& \int \mathrm{d} \mu_{S O(3)}(y)[2 n]_{y}[1]_{y}^{m}[2 k+1]_{y}= \begin{cases}T_{2 n+1}\left(m, \frac{1}{2} m+n-k-\frac{1}{2}\right) & \text { if } m \text { is odd, } \\
0 & \text { if } m \text { is even, }\end{cases} \\
& \int \mathrm{d} \mu_{S O(3)}(y)[2 n+1]_{y}[1]_{y}^{m}[2 k+1]_{y}= \begin{cases}T_{2 n+2}\left(m, \frac{1}{2} m+n-k\right) & \text { if } m \text { is even, } \\
0 & \text { if } m \text { is odd, }\end{cases}
\end{aligned}
$$

where $T_{p}(m, k)$ is defined as above and the zeros once again confirm the selection rule (4.49).

The multiplicities of $\llbracket 2 n, 2 Q+1 \rrbracket$ are given by

$$
\begin{aligned}
M\left(\left.\chi_{\mathrm{R}}^{\mathcal{N}_{4 d}=1}\right|_{\mathrm{GSO}}, \llbracket 2 n, 2 Q+1 \rrbracket, q\right) \\
=\sum_{k=0}^{\infty} \sum_{m=0}^{\infty} \sum_{p=-\infty}^{Q+m}(-1)^{Q-m-p+1} F_{k, p}^{\mathrm{R}}(q) \\
\quad \times\left(\begin{array}{c}
Q+m-p+1 \\
2 m+1
\end{array}\right) T_{2 n+1}(2 m+1, m+n-k) .
\end{aligned}
$$

The multiplicities of $\llbracket 2 n+1,2 Q \rrbracket$ are given by 


$$
\begin{aligned}
M & \left(\left.\chi_{\mathrm{R}}^{\mathcal{N}_{4 d}=1}\right|_{\mathrm{GSO}}, \llbracket 2 n+1,2 Q \rrbracket, q\right) \\
= & \sum_{k=0}^{\infty} \sum_{m=0}^{\infty} \sum_{p=-\infty}^{Q+m}(-1)^{Q-m-p} F_{k, p}^{\mathrm{R}}(q) \\
& \times\left(\begin{array}{c}
Q+m-p \\
2 m
\end{array}\right) T_{2 n+2}(2 m, m+n-k) .
\end{aligned}
$$

\subsubsection{Combining the NS and $R$ sectors}

Now we can assemble the NS and R sector results to obtain the full multiplicities of the representation $\llbracket n, Q \rrbracket$ in $\chi^{\mathcal{N}_{4 d}=1}(q ; y, s)$. First, it is clear that

$$
G_{2 n, 2 Q}(q)=G_{2 n+1,2 Q+1}(q)=0 .
$$

The non-zero multiplicities of $\llbracket 2 n, 2 Q+1 \rrbracket$ and $\llbracket 2 n+1,2 Q \rrbracket$ are most conveniently presented in terms of the shorthands

$$
\begin{aligned}
& \mathfrak{M}_{\llbracket 2 n, 2 Q+1 \rrbracket}(m, p, k ; q) \\
& :=(-1)^{Q-m-p}\left[F_{k, p}^{\mathrm{NS}}(q)\left(\begin{array}{c}
Q+m-p \\
2 m
\end{array}\right) T_{2 n+1}(2 m, m+n-k)\right. \\
& \left.-F_{k, p}^{\mathrm{R}}(q)\left(\begin{array}{c}
Q+m-p+1 \\
2 m+1
\end{array}\right) T_{2 n+1}(2 m+1, m+n-k)\right], \\
& \mathfrak{M}_{\llbracket 2 n+1,2 Q \rrbracket}(m, p, k ; q) \\
& :=(-1)^{Q-m-p}\left[F_{k, p}^{\mathrm{NS}}(q)\left(\begin{array}{c}
Q+m-p \\
2 m+1
\end{array}\right) T_{2 n+2}(2 m+1, m+n+1-k)\right. \\
& \left.+F_{k, p}^{\mathrm{R}}(q)\left(\begin{array}{c}
Q+m-p \\
2 m
\end{array}\right) T_{2 n+2}(2 m, m+n-k)\right]
\end{aligned}
$$

for the contributions $\mathfrak{M}_{\llbracket \cdot, \cdot]}(m, p, k ; q)$ of individual terms in the $m, p, k$ triple sum to the multiplicity generating function. The result for $\llbracket 2 n, 2 Q+1 \rrbracket$ supermultiplets is

$$
\begin{aligned}
G_{2 n, 2 Q+1}(q) & \\
= & \sum_{k=0}^{\infty} \sum_{m=0}^{\infty} \sum_{p=-\infty}^{Q+m} \mathfrak{M}_{\llbracket 2 n, 2 Q+1 \rrbracket}(m, p, k ; q) \\
= & \sum_{k=0}^{\infty} \sum_{m=0}^{\infty}\left[\sum_{p=0}^{\infty}\left\{\mathfrak{M}_{\llbracket 2 n, 2 Q+1 \rrbracket}(m,-p-1, k ; q)+\mathfrak{M}_{\llbracket 2 n, 2 Q+1 \rrbracket}(m+p, p, k ; q)\right\}\right. \\
& \left.+\sum_{p=0}^{Q-1} \mathfrak{M}_{\llbracket 2 n, 2 Q+1 \rrbracket}(m, m+p+1, k ; q)\right]
\end{aligned}
$$

whereas the multiplicities of $\llbracket 2 n+1,2 Q \rrbracket$ are given by

$$
\begin{aligned}
& G_{2 n+1,2 Q}(q) \\
& \quad=\sum_{k=0}^{\infty} \sum_{m=0}^{\infty} \sum_{p=-\infty}^{Q+m} \mathfrak{M}_{\llbracket 2 n+1,2 Q \rrbracket}(m, p, k ; q)
\end{aligned}
$$




$$
\begin{aligned}
= & \sum_{k=0}^{\infty} \sum_{m=0}^{\infty}\left[\sum_{p=0}^{\infty}\left\{\mathfrak{M}_{\llbracket 2 n+1,2 Q \rrbracket}(m,-p-1, k ; q)+\mathfrak{M}_{\llbracket 2 n+1,2 Q \rrbracket}(m+p, p, k ; q)\right\}\right. \\
& \left.+\sum_{p=0}^{Q-1} \mathfrak{M}_{\llbracket 2 n+1,2 Q \rrbracket}(m, m+p+1, k ; q)\right] .
\end{aligned}
$$

\subsection{Asymptotic analysis for the multiplicities}

This subsection is devoted to the multiplicity generating function $G_{n, Q}(q)$ in the limit $n \rightarrow \infty$. We shall present analytic expressions for their $n \rightarrow \infty$ asymptotics whose derivation is deferred to Appendix C. The method essentially relies on identifying the dominant contribution to the triple sums in (4.61) and (4.62). The end result for multiplicity generating functions $G_{n, Q}(q)$ reads

$$
\begin{aligned}
G_{2 n+1,2 Q}(q) \sim & \frac{(1-q)^{2} q^{n-\frac{3}{2}}}{2(q ; q)_{\infty}^{6}} \mathcal{F}(q, Q), \quad n \rightarrow \infty \\
G_{2 n, 2 Q+1}(q) \sim & \frac{(1-q)^{2} q^{n-\frac{3}{2}}}{2(q ; q)_{\infty}^{6}(1+q)} \\
& \times\left[\frac{q^{(Q+1)^{2}+\frac{1}{4}}(1-q)}{\left(1+q^{Q}\right)\left(1+q^{Q+1}\right)} \vartheta_{2}(1, q)^{2}-\mathcal{F}(q, Q)-\mathcal{F}(q, Q+1)\right]
\end{aligned}
$$

with the function $\mathcal{F}(q, Q)$ given by

$$
\begin{aligned}
& \mathcal{F}(q, Q) \\
& =\vartheta_{2}(1, q)^{2}\left[q^{1-Q_{u}}(\sqrt{q}, Q)+(-1)^{Q}(1-q)\left(v_{1}(\sqrt{q}, Q)+q^{-1 / 4} w_{1}(\sqrt{q}, Q)\right)\right]
\end{aligned}
$$

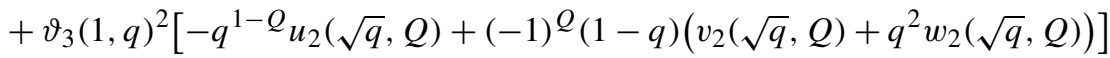

$$
\begin{aligned}
& +\vartheta_{4}(1, q)^{2}\left[q^{1-Q_{u}}(-\sqrt{q}, Q)-(-1)^{Q}(1-q)\left(v_{2}(-\sqrt{q}, Q)+q^{2} w_{2}(-\sqrt{q}, Q)\right)\right] \text {. }
\end{aligned}
$$

The three pairs of functions $u_{i}, v_{i}$ and $w_{i}$ correspond to the three summations in (4.61) and (4.62):

$$
\begin{aligned}
& u_{1}(q, Q)=\sum_{p=0}^{\infty} q^{2\left(p+\frac{3}{2}\right)^{2}} \frac{1-q^{4 p+4 Q+6}}{\left(1+q^{2 p+2}\right)\left(1+q^{2 p+4}\right)}, \\
& u_{2}(q, Q)=\sum_{p=0}^{\infty} q^{2(p+1)^{2}} \frac{1-q^{4 p+4 Q+4}}{\left(1+q^{2 p+1}\right)\left(1+q^{2 p+3}\right)}, \\
& v_{1}(q, Q)=\sum_{p=0}^{\lfloor Q / 2\rfloor} \frac{q^{2\left(p-\frac{1}{2}\right)^{2}}\left(1+q^{2}\right)^{2 p}}{\left(1+q^{2 p-2}\right)\left(1+q^{2 p}\right)}\left(\begin{array}{c}
Q \\
2 p
\end{array}\right){ }_{3} F_{2}\left[\begin{array}{c}
1, Q+1,2 p-Q \\
p+1 / 2, p+1
\end{array} ; \frac{(1+q)^{2}}{4 q}\right], \\
& v_{2}(q, Q)=\sum_{p=0}^{\lfloor Q / 2\rfloor} \frac{(1+q) q^{2 p^{2}}\left(1+q^{2}\right)^{2 p}}{\left(1+q^{2 p-1}\right)\left(1+q^{2 p+1}\right)}\left(\begin{array}{c}
Q \\
2 p+1
\end{array}\right){ }_{3} F_{2}\left[\begin{array}{c}
1, Q+1,2 p+1-Q \\
p+1, p+3 / 2
\end{array} ; \frac{(1+q)^{2}}{4 q}\right],
\end{aligned}
$$




$$
\begin{aligned}
& w_{1}(q, Q)=\sum_{m=0}^{\infty} \sum_{p=0}^{Q-1} \frac{(-1)^{p+1} q^{1+2(1+m+p)^{2}-2 m}\left(1+q^{2}\right)^{2 m}\left(\begin{array}{c}
Q-1-p \\
2 m
\end{array}\right)}{\left(1+q^{2(m+p)}\right)\left(1+q^{2(1+m+p)}\right)}, \\
& w_{2}(q, Q)=q^{-\frac{9}{2}} \sum_{m=0}^{\infty} \sum_{p=0}^{Q-1} \frac{(-1)^{p+1} q^{2\left(m+p+\frac{3}{2}\right)^{2}-2 m}\left(1+q^{2}\right)^{2 m+1}\left(\begin{array}{c}
Q-1-p \\
1+2 m
\end{array}\right)}{\left(1+q^{1+2 m+2 p}\right)\left(1+q^{3+2 m+2 p}\right)} .
\end{aligned}
$$

Note that the leading orders in the power series are

$$
G_{2 n+1,2 Q}(q) \sim q^{n+Q(Q+2)}, \quad G_{2 n, 2 Q+1}(q) \sim q^{n+Q^{2}+3 Q+1}, \quad q \rightarrow 0,
$$

i.e. the supermultiplet $\llbracket 2 n+1,2 Q \rrbracket$ firstly occurs at mass level $n+Q(Q+2)$ whereas the $\llbracket 2 n, 2 Q+1 \rrbracket$ multiplet firstly occurs at mass level $n+Q^{2}+3 Q+1$.

For reference, we list the leading $q$ powers for the $G_{n \rightarrow \infty, Q}$ regime for some small values of the $U(1)_{R}$ charge, obtained by expansion of (4.63) and (4.64): firstly for even values $Q \in 2 \mathbb{N}_{0}$

$$
\begin{aligned}
G_{2 n+1,0}(q) \sim & q^{n}\left(1+q+7 q^{2}+19 q^{3}+53 q^{4}+133 q^{5}+328 q^{6}+752 q^{7}\right. \\
& \left.+1689 q^{8}+3635 q^{9}+O\left(q^{10}\right)\right), \\
G_{2 n+1,2}(q) \sim & q^{n+3}\left(2+8 q+24 q^{2}+73 q^{3}+187 q^{4}+467 q^{5}+1090 q^{6}\right. \\
& \left.+2457 q^{7}+5314 q^{8}+O\left(q^{9}\right)\right), \\
G_{2 n+1,4}(q) \sim & q^{n+8}\left(2+10 q+36 q^{2}+110 q^{3}+306 q^{4}+773 q^{5}+1861 q^{6}\right. \\
& \left.+4245 q^{7}+9327 q^{8}+O\left(q^{9}\right)\right), \\
G_{2 n+1,6}(q) \sim & q^{n+15}\left(2+10 q+38 q^{2}+124 q^{3}+352 q^{4}+928 q^{5}+2282 q^{6}\right. \\
& \left.+5335 q^{7}+O\left(q^{8}\right)\right),
\end{aligned}
$$

and secondly for odd values $Q \in 2 \mathbb{N}-1$

$$
\begin{aligned}
G_{2 n, 1}(q) \sim & q^{n+1}\left(3+5 q+22 q^{2}+53 q^{3}+150 q^{4}+345 q^{5}+836 q^{6}\right. \\
& \left.+1824 q^{7}+4011 q^{8}+O\left(q^{9}\right)\right), \\
G_{2 n, 3}(q) \sim & q^{n+5}\left(4+11 q+46 q^{2}+117 q^{3}+331 q^{4}+784 q^{5}\right. \\
& \left.+1876 q^{6}+4133 q^{7}+O\left(q^{8}\right)\right), \\
G_{2 n, 5}(q) \sim & q^{n+11}\left(4+12 q+55 q^{2}+150 q^{3}+437 q^{4}+1078 q^{5}\right. \\
& \left.+2640 q^{6}+5951 q^{7}+O\left(q^{8}\right)\right), \\
G_{2 n, 7}(q) \sim & q^{n+19}\left(4+12 q+56 q^{2}+159 q^{3}+474 q^{4}+1197 q^{5}\right. \\
& \left.+2994 q^{6}+6882 q^{7}+O\left(q^{8}\right)\right) .
\end{aligned}
$$

Note that the general formula greatly simplifies at $U(1)_{R}$ charges $Q=0$ and $Q=1$,

$$
\begin{aligned}
G_{2 n+1,0}(q) \sim & \frac{q^{n}}{(q ; q)_{\infty}^{6}}\left\{\frac { 1 } { 2 } ( 1 - q ) ^ { 2 } q ^ { - \frac { 1 } { 2 } } \left(u_{1}(\sqrt{q}) \vartheta_{2}(1, q)^{2}\right.\right. \\
& \left.-\left[u_{2}(\sqrt{q}) \vartheta_{3}(1, q)^{2}-u_{2}(-\sqrt{q}) \vartheta_{4}(1, q)^{2}\right]\right) \\
& \left.+\frac{1}{4} \frac{(1-q)^{3}}{1+q} q^{-\frac{1}{4}} \vartheta_{2}(1, q)^{2}\right\}, \quad n \rightarrow \infty,
\end{aligned}
$$




$$
\begin{aligned}
G_{2 n, 1}(q) \sim & \frac{(1-q)^{3} q^{n+1}}{4(q ; q)_{\infty}^{6}}\left[q^{-\frac{5}{2}}\left\{\frac{\vartheta_{3}(1, q)^{2}}{\left(1+q^{-\frac{1}{2}}\right)\left(1+q^{\frac{1}{2}}\right)}-\frac{\vartheta_{4}(1, q)^{2}}{\left(1-q^{-\frac{1}{2}}\right)\left(1-q^{\frac{1}{2}}\right)}\right\}\right. \\
& -\frac{1}{2} q^{-\frac{9}{4}} \vartheta_{2}(1, q)^{2}-q^{-\frac{5}{2}} \frac{1+q}{1-q}\left(u_{1}(\sqrt{q}) \vartheta_{2}(1, q)^{2}\right. \\
& \left.\left.-\left[u_{2}(\sqrt{q}) \vartheta_{3}(1, q)^{2}-u_{2}(-\sqrt{q}) \vartheta_{4}(1, q)^{2}\right]\right)\right]
\end{aligned}
$$

where $u_{i}(q) \equiv u_{i}(q ; 0)$, see the first subsection of Appendix C.

\subsection{Empirical approach to $\mathcal{N}_{4 d}=1$ asymptotic patterns}

In the previous subsection, we have derived the large spin asymptotics for multiplicity generating functions $G_{k, Q}(q)$ of individual $\mathcal{N}_{4 d}=1$ multiplets (at finite $Q$ while $k \rightarrow \infty$ ), the main results being (4.63) and (4.64). The asymptotic formulae can be viewed as the supersymmetric generalization of truncating the infinite sum expression (2.10) for the $S O(3)$ multiplicity generating function in the $d=4$ bosonic partition function to its $n=1$ term. In [8], this $n=1$ term is interpreted as the leading (additive) Regge trajectory of unit slope, followed by an infinite tower of sister trajectories of fractional slope and alternating sign.

Let us borrow some notation from Eq. (6.2) of [8] and expand the $G_{k, Q}(q)$ in an infinite series of trajectories $\tau_{\ell}$ :

$$
\begin{aligned}
G_{2 n+1,2 Q}(q) & =q^{n} \tau_{1}^{2 Q}(q)-q^{2 n} \tau_{2}^{2 Q}(q)+q^{3 n} \tau_{3}^{2 Q}(q)-\cdots \\
& =\sum_{\ell=1}^{\infty}(-1)^{\ell-1} q^{\ell n} \tau_{\ell}^{2 Q}(q), \\
G_{2 n, 2 Q+1}(q) & =q^{n} \tau_{1}^{2 Q+1}(q)-q^{2 n} \tau_{2}^{2 Q+1}(q)+q^{3 n} \tau_{3}^{2 Q+1}(q)-\cdots \\
& =\sum_{\ell=1}^{\infty}(-1)^{\ell-1} q^{\ell n} \tau_{\ell}^{2 Q+1}(q) .
\end{aligned}
$$

It is not obvious that the patterns observed in [8] for non-supersymmetric theories persist for the counting of super-Poincaré multiplets, i.e. that the spacetime partition functions of the reference preserve the nested structure in (4.74) after multiplication with the internal characters. At any rate, all our $\mathcal{N}_{4 d}=1$ data suggests that both of $\tau_{\ell}^{2 Q}(q)$ and $\tau_{\ell}^{2 Q+1}(q)$ are power series in $q$ with non-negative coefficients. Our analytic results (4.63) and (4.64) identify the first coefficient functions $\tau_{1}(q)$ in (4.74):

$$
\begin{aligned}
\tau_{1}^{2 Q}(q)=q & \frac{(1-q)^{2} q^{-\frac{3}{2}}}{2(q ; q)_{\infty}^{6}} \mathcal{F}(q, Q), \\
\tau_{1}^{2 Q+1}(q)= & \frac{(1-q)^{2} q^{-\frac{3}{2}}}{2(q ; q)_{\infty}^{6}(1+q)} \\
& \times\left[\frac{q^{(Q+1)^{2}+\frac{1}{4}}(1-q)}{\left(1+q^{Q}\right)\left(1+q^{Q+1}\right)} \vartheta_{2}(1, q)^{2}-\mathcal{F}(q, Q)-\mathcal{F}(q, Q+1)\right] .
\end{aligned}
$$

The methods presented in Appendix $C$ and applied in the previous subsection are not suitable to extract subleading Regge trajectories $\tau_{\ell \geqslant 2}(q)$, i.e. $\mathcal{N}_{4 d}=1$ analogues of $n \geqslant 2$ terms in the sum (2.10). Instead, we shall rely on an empirical approach, more specifically on explicit results 
obtained from a supercharacter expansion of the partition function (4.4) up to the 25th mass level.

As an illustrative example, let us first of all investigate the family of $Q=0$ supermultiplets: The following Table 3 gathers $\llbracket 2 n+1,0 \rrbracket$ multiplicities in the first 25 levels. Numbers marked in red directly correspond to the leading trajectory $\tau_{1}^{0}(q)$ whereas those in blue are additionally affected by the subleading trajectory $\tau_{2}^{0}(q)$. Given the leading trajectories (4.75), our data from Table 3 can be used to determine the following subleading behavior for $Q=0$ multiplets:

$$
\begin{aligned}
G_{2 n+1,0}(q) \sim & q^{n}\left(1+q+7 q^{2}+19 q^{3}+53 q^{4}+133 q^{5}+328 q^{6}\right. \\
& +752 q^{7}+1689 q^{8}+3635 q^{9}+7642 q^{10}+15608 q^{11}+31235 q^{12} \\
& +61115 q^{13}+117513 q^{14}+221927 q^{15}+412778 q^{16} \\
& +756372 q^{17}+1367753 q^{18}+2441849 q^{19}+4309132 q^{20} \\
& +7520092 q^{21}+12989357 q^{22}+22216885 q^{23}+37651970 q^{24} \\
& \left.+63252874 q^{25}+\cdots\right)-q^{2 n+1}\left(2+8 q+26 q^{2}+78 q^{3}+214 q^{4}+548 q^{5}\right. \\
& +1330 q^{6}+3080 q^{7}+6872 q^{8}+14832 q^{9}+31102 q^{10}+63574 q^{11} \\
& \left.+127020 q^{12}+248590 q^{13}+477504 q^{14}+\cdots\right) \\
& +q^{3 n+1}\left(1+4 q+19 q^{2}+61 q^{3}+187 q^{4}+503 q^{5}+1294 q^{6}+3113 q^{7}\right. \\
& \left.+7217 q^{8}+16036 q^{9}+34584 q^{10}+\cdots\right) \\
& -q^{4 n+2}\left(2+10 q+38 q^{2}+124 q^{3}+364 q^{4}+978 q^{5}+2476 q^{6}+\cdots\right) \\
& +q^{5 n+2}\left(1+4 q+21 q^{2}+72 q^{3}+\cdots\right)+\cdots, \quad n \rightarrow \infty .
\end{aligned}
$$

The first term linear in $q^{n}$ simply reproduces (4.72) for $\tau_{1}^{Q=0}(q)$ whereas higher powers of $q^{n}$ allow to read off subleading $\tau_{\ell \geqslant 2}^{Q=0}(q)$ to certain order in $q$ :

$$
\begin{aligned}
\tau_{2}^{Q=0}(q)= & q\left(2+8 q+26 q^{2}+78 q^{3}+214 q^{4}+548 q^{5}+1330 q^{6}+3080 q^{7}+6872 q^{8}\right. \\
& +14832 q^{9}+31102 q^{10}+63574 q^{11}+127020 q^{12} \\
& \left.+248590 q^{13}+477504 q^{14}+\cdots\right), \\
\tau_{3}^{Q=0}(q)= & q\left(1+4 q+19 q^{2}+61 q^{3}+187 q^{4}+503 q^{5}+1294 q^{6}+3113 q^{7}\right. \\
& \left.+7217 q^{8}+16036 q^{9}+34584 q^{10}+\cdots\right), \\
\tau_{4}^{Q=0}(q)= & q^{2}\left(2+10 q+38 q^{2}+124 q^{3}+364 q^{4}+978 q^{5}+2476 q^{6}+\cdots\right), \\
\tau_{5}^{Q=0}(q)= & q^{2}\left(1+4 q+21 q^{2}+72 q^{3}+\cdots\right) .
\end{aligned}
$$

Determining higher order terms in the $\tau_{\ell \geqslant 2}^{Q=0}(q)$ would require $\mathcal{O}\left(q^{26}\right)$ parts of (4.4), this is where we stopped the explicit evaluation.

Similarly, the $\llbracket 2 n+1,2 \rrbracket$ and $\llbracket 2 n, 1 \rrbracket$ multiplicities up to level $q^{25}$ as tabulated in Appendix B.1 determine the associated $\tau_{\ell}(q)$ coefficients to the following orders:

$$
\begin{aligned}
\tau_{2}^{Q=2}(q)= & q^{3}\left(2+11 q+37 q^{2}+114 q^{3}+319 q^{4}+822 q^{5}+2000 q^{6}+4645 q^{7}\right. \\
& \left.+10354 q^{8}+22317 q^{9}+46702 q^{10}+95210 q^{11}+189656 q^{12}+\cdots\right),
\end{aligned}
$$


Table 3

$\mathcal{N}_{4 d}=1$ multiplets at $U(1)_{R}$ charge $Q=0$.

\begin{tabular}{|c|c|c|c|c|c|c|c|c|c|c|c|}
\hline$\alpha^{\prime} m^{2}$ & $\# \llbracket 1,0 \rrbracket$ & $\# \llbracket 3,0 \rrbracket$ & $\# \llbracket 5,0 \rrbracket$ & 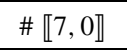 & 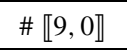 & $\# \llbracket 11,0 \rrbracket$ & $\# \llbracket 13,0 \rrbracket$ & $\# \llbracket 15,0 \rrbracket$ & $\# \llbracket 17,0 \rrbracket$ & $\# \llbracket 19,0 \rrbracket$ & 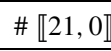 \\
\hline 1 & 0 & 1 & 0 & & & & & & & & \\
\hline 2 & 2 & 1 & 1 & 0 & & & & & & & \\
\hline 3 & 5 & 5 & 1 & 1 & 0 & & & & & & \\
\hline 4 & 12 & 12 & 7 & 1 & 1 & 0 & & & & & \\
\hline 5 & 28 & 31 & 17 & 7 & 1 & 1 & 0 & & & & \\
\hline 6 & 64 & 72 & 45 & 19 & 7 & 1 & 1 & 0 & & & \\
\hline 7 & 136 & 166 & 108 & 51 & 19 & 7 & 1 & 1 & 0 & & \\
\hline 8 & 289 & 357 & 254 & 125 & 53 & 19 & 7 & 1 & 1 & 0 & \\
\hline 9 & 588 & 757 & 557 & 302 & 131 & 53 & 19 & 7 & 1 & 1 & 0 \\
\hline 10 & 1175 & 1548 & 1200 & 675 & 320 & 133 & 53 & 19 & 7 & 1 & 1 \\
\hline 11 & 2293 & 3100 & 2482 & 1479 & 726 & 326 & 133 & 53 & 19 & 7 & 1 \\
\hline 12 & 4399 & 6053 & 5028 & 3106 & 1611 & 744 & 328 & 133 & 53 & 19 & 7 \\
\hline 13 & 8267 & 11620 & 9910 & 6373 & 3422 & 1663 & 750 & 328 & 133 & 53 & 19 \\
\hline 14 & 15325 & 21855 & 19173 & 12713 & 7098 & 3557 & 1681 & 752 & 328 & 133 & 53 \\
\hline 15 & 27949 & 40496 & 36322 & 24856 & 14297 & 7428 & 3609 & 1687 & 752 & 328 & 133 \\
\hline 16 & 50306 & 73846 & 67720 & 47539 & 28216 & 15061 & 7564 & 3627 & 1689 & 752 & 328 \\
\hline 17 & 89367 & 132860 & 124161 & 89401 & 54430 & 29909 & 15394 & 7616 & 3633 & 1689 & 752 \\
\hline 18 & 156930 & 235871 & 224479 & 165210 & 103182 & 58054 & 30687 & 15530 & 7634 & 3635 & 1689 \\
\hline 19 & 272424 & 413879 & 400257 & 300837 & 192109 & 110702 & 59786 & 31021 & 15582 & 7640 & 3635 \\
\hline 20 & 468130 & 717909 & 705032 & 539962 & 352279 & 207282 & 114437 & 60567 & 31157 & 15600 & 7642 \\
\hline 21 & 796410 & 1232463 & 1227214 & 956883 & 636445 & 382179 & 215074 & 116183 & 60901 & 31209 & 15606 \\
\hline 22 & 1342531 & 2094716 & 2113394 & 1674933 & 1134836 & 694090 & 398007 & 218848 & 116965 & 61037 & 31227 \\
\hline 23 & 2243232 & 3527456 & 3602086 & 2899342 & 1997955 & 1243836 & 725457 & 405910 & 220597 & 117299 & 61089 \\
\hline 24 & 3717405 & 5887668 & 6081317 & 4965411 & 3477396 & 2200438 & 1304682 & 741559 & 409698 & 221379 & 117435 \\
\hline 25 & 6111615 & 9745995 & 10173766 & 8420331 & 5986079 & 3847540 & 2316123 & 1336712 & 749501 & 411448 & 221713 \\
\hline
\end{tabular}




$$
\begin{aligned}
\tau_{3}^{Q=2}(q)= & q^{3}\left(2+8 q+33 q^{2}+104 q^{3}+310 q^{4}+826 q^{5}+2093 q^{6}+4991 q^{7}\right. \\
& \left.+11454 q^{8}+\cdots\right), \\
\tau_{4}^{Q=2}(q)= & q^{3}\left(1+5 q+22 q^{2}+77 q^{3}+237 q^{4}+664 q^{5}+\cdots\right), \\
\tau_{5}^{Q=2}(q)= & q^{4}\left(3+12 q+49 q^{2}+\cdots\right), \\
\tau_{2}^{Q=1}(q)= & 1+4 q+15 q^{2}+50 q^{3}+143 q^{4}+379 q^{5}+947 q^{6}+2244 q^{7}+5103 q^{8} \\
& +11196 q^{9}+23804 q^{10}+49252 q^{11}+99465 q^{12}+196522 q^{13} \\
& +380719 q^{14}+\cdots, \\
\tau_{3}^{Q=1}(q)= & 1+5 q+22 q^{2}+70 q^{3}+212 q^{4}+568 q^{5}+1458 q^{6}+3496 q^{7} \\
& +8093 q^{8}+17936 q^{9}+\cdots, \\
\tau_{4}^{Q=1}(q)=1 & +6 q+24 q^{2}+83 q^{3}+252 q^{4}+698 q^{5}+\cdots, \\
\tau_{5}^{Q=1}(q)= & 1+6 q+25 q^{2}+\cdots .
\end{aligned}
$$

Note that the analytic result (4.63) for $\tau_{1}^{2}(q), \tau_{1}^{1}(q)$ was used as an extra input, in addition to the explicit results for the first 25 mass level, to make a few more orders of the subleading $\tau_{\ell \geqslant 2}(q)$ accessible. Some more leading and subleading $\tau_{\ell}^{Q}$ for larger values of $Q$ are given in AUXILIARY FILE 2.

\section{Spectra in compactifications with 8 supercharges}

In six-dimensional Minkowski space, the minimal realization of SUSY involves eight supercharges. They form two left-handed Weyl spinors of $S O(6)$ which are related through an $S U(2)_{R}$ R symmetry. Our notation for such minimally supersymmetric theories in $d=6$ is $\mathcal{N}_{6 d}=(1,0)$. Superstring compactification subject to $\mathcal{N}_{6 d}=(1,0)$ SUSY are described by a universal SCFT sector with $c=6$ and $\mathcal{N}_{2 d}=4$ SUSY on the worldsheet, see Section 3.2 for details. In addition, the SCFT introduces $S O(5)$ quantum numbers for the massive string states through a six-dimensional spacetime sector for which the methods of Sections 2.4 and 2.5 are applicable.

The fundamental multiplet of $\mathcal{N}_{6 d}=(1,0)$ theories consists of $8+8$ states

$$
Z\left(\mathcal{N}_{6 d}=(1,0)\right):=[1,0]+[2]_{R}+[1]_{R}[0,1] .
$$

where $[p]_{R}$ is the character of the $(p+1)$-dimensional representation of $S U(2)_{R}$. Generic multiplets follow through the tensor product with some $S O(5) \times S U(2)_{R}$ representation with little group quantum numbers $\left[n_{1}, n_{2}\right]$ and R-symmetry content $[k]_{R}$. This leads to the general supercharacter

$$
\llbracket n_{1}, n_{2} ; p \rrbracket:=Z\left(\mathcal{N}_{6 d}=(1,0)\right) \cdot[p]_{R}\left[n_{1}, n_{2}\right] .
$$

The partition function capturing the universal spectrum of six-dimensional $\mathcal{N}_{6 d}=(1,0)$ compactifications is obtained through a GSO projected product of internal $\chi_{\ldots}^{\mathcal{N}_{2 d}=4, c=6}(q ; r)$ characters (with $S U(2)_{R}$ fugacity $r$ ) defined by (3.7) as well as (3.10) and $S O(5)$ spacetime characters (2.69) and (2.70). The GSO projection removes half odd integer mass leaves from the NS sector and enforces the R spin field to be a left-handed $S O(6)$ spinor, therefore: 
Table 4

$\mathcal{N}_{6 d}=(1,0)$ multiplets occurring up to mass level 6.

\begin{tabular}{|c|c|}
\hline$\alpha^{\prime} m^{2}$ & representations of $\mathcal{N}_{6 d}=(1,0)$ super-Poincaré \\
\hline 1 & $\llbracket 1,0 ; 0 \rrbracket$ \\
\hline 2 & $\llbracket 2,0 ; 0 \rrbracket+\llbracket 0,2 ; 0 \rrbracket+\llbracket 0,1 ; 1 \rrbracket$ \\
\hline 3 & $\llbracket 3,0 ; 0 \rrbracket+2 \llbracket 1,0 ; 0 \rrbracket+\llbracket 0,0 ; 0 \rrbracket+\llbracket 1,2 ; 0 \rrbracket+\llbracket 0,2 ; 0 \rrbracket+\llbracket 0,0 ; 2 \rrbracket+2 \llbracket 1,1 ; 1 \rrbracket+\llbracket 0,1 ; 1 \rrbracket$ \\
\hline 4 & $\begin{array}{l}\llbracket 4,0 ; 0 \rrbracket+3 \llbracket 2,0 ; 0 \rrbracket+2 \llbracket 1,0 ; 0 \rrbracket+2 \llbracket 0,0 ; 0 \rrbracket+\llbracket 2,2 ; 0 \rrbracket+2 \llbracket 1,2 ; 0 \rrbracket+4 \llbracket 0,2 ; 0 \rrbracket+2 \llbracket 1,0 ; 2 \rrbracket \\
\quad+\llbracket 0,2 ; 2 \rrbracket+3 \llbracket 1,1 ; 1 \rrbracket+4 \llbracket 0,1 ; 1 \rrbracket+2 \llbracket 2,1 ; 1 \rrbracket\end{array}$ \\
\hline 5 & $\begin{array}{l}\llbracket 5,0 ; 0 \rrbracket+3 \llbracket 3,0 ; 0 \rrbracket+4 \llbracket 2,0 ; 0 \rrbracket+9 \llbracket 1,0 ; 0 \rrbracket+3 \llbracket 0,0 ; 0 \rrbracket+\llbracket 3,2 ; 0 \rrbracket+2 \llbracket 2,2 ; 0 \rrbracket+7 \llbracket 1,2 ; 0 \rrbracket \\
\quad+6 \llbracket 0,2 ; 0 \rrbracket+\llbracket 0,4 ; 0 \rrbracket+3 \llbracket 2,0 ; 2 \rrbracket+3 \llbracket 1,0 ; 2 \rrbracket+3 \llbracket 0,0 ; 2 \rrbracket+\llbracket 1,2 ; 2 \rrbracket+3 \llbracket 0,2 ; 2 \rrbracket+2 \llbracket 3,1 ; 1 \rrbracket \\
\quad+4 \llbracket 2,1 ; 1 \rrbracket+9 \llbracket 1,1 ; 1 \rrbracket+8 \llbracket 0,1 ; 1 \rrbracket+\llbracket 1,3 ; 1 \rrbracket+4 \llbracket 0,3 ; 1 \rrbracket+\llbracket 0,1 ; 3 \rrbracket\end{array}$ \\
\hline 6 & $\begin{array}{l}\llbracket 6,0 ; 0 \rrbracket+\llbracket 4,2 ; 0 \rrbracket+2 \llbracket 4,1 ; 1 \rrbracket+3 \llbracket 4,0 ; 0 \rrbracket+2 \llbracket 3,2 ; 0 \rrbracket+4 \llbracket 3,1 ; 1 \rrbracket+3 \llbracket 3,0 ; 2 \rrbracket \\
\quad+5 \llbracket 3,0 ; 0 \rrbracket+\llbracket 2,3 ; 1 \rrbracket+\llbracket 2,2 ; 2 \rrbracket+8 \llbracket 2,2 ; 0 \rrbracket+12 \llbracket 2,1 ; 1 \rrbracket+4 \llbracket 2,0 ; 2 \rrbracket+14 \llbracket 2,0 ; 0 \rrbracket \\
\quad+\llbracket 1,4 ; 0 \rrbracket+5 \llbracket 1,3 ; 1 \rrbracket+6 \llbracket 1,2 ; 2 \rrbracket+13 \llbracket 1,2 ; 0 \rrbracket+2 \llbracket 1,1 ; 3 \rrbracket+23 \llbracket 1,1 ; 1 \rrbracket+9 \llbracket 1,0 ; 2 \rrbracket \\
\quad+12 \llbracket 1,0 ; 0 \rrbracket+4 \llbracket 0,4 ; 0 \rrbracket+9 \llbracket 0,3 ; 1 \rrbracket+9 \llbracket 0,2 ; 2 \rrbracket+19 \llbracket 0,2 ; 0 \rrbracket+3 \llbracket 0,1 ; 3 \rrbracket \\
\quad+18 \llbracket 0,1 ; 1 \rrbracket+4 \llbracket 0,0 ; 2 \rrbracket+8 \llbracket 0,0 ; 0 \rrbracket\end{array}$ \\
\hline
\end{tabular}

$$
\begin{aligned}
& \chi^{\mathcal{N}_{6 d}=(1,0)}(q ; \boldsymbol{y}, r)=\left.\chi_{\mathrm{NS}}^{\mathcal{N}_{6 d}=(1,0)}\right|_{\mathrm{GSO}}(q ; \boldsymbol{y}, r)+\left.\chi_{\mathrm{R}}^{\mathcal{N}_{6 d}=(1,0)}\right|_{\mathrm{GSO}}(q ; \boldsymbol{y}, r), \\
& \left.\chi_{\mathrm{NS}}^{\mathcal{N}_{6 d}=(1,0)}\right|_{\mathrm{GSO}}(q ; \boldsymbol{y}, r)=\frac{1}{2} q^{-\frac{1}{2}}\left[\chi_{\mathrm{NS}}^{S O(5)}(q ; \boldsymbol{y}) \chi_{\mathrm{NS}, h=0, \ell=0}^{\mathcal{N}_{2 d}=4, c=6}(q ; r)\right. \\
& \left.-\chi_{\mathrm{NS}}^{S O(5)}\left(e^{2 \pi i} q ; \boldsymbol{y}\right) \chi_{\mathrm{NS}, h=0, \ell=0}^{\mathcal{N}_{2 d}=4, c=6}\left(e^{2 \pi i} q ; r\right)\right] \text {, } \\
& \left.\chi_{\mathrm{R}}^{\mathcal{N}_{6 d}=(1,0)}\right|_{\mathrm{GSO}}(q ; \boldsymbol{y}, r)=\frac{1}{2} \chi_{\mathrm{R}}^{S O(5)}(q ; \boldsymbol{y}) \chi_{\mathrm{R}, h=1 / 4, \ell=1 / 2}^{\mathcal{N}_{2 d}=4, c=6}(q ; r) .
\end{aligned}
$$

The power series expansion of (5.3) starts as 23

$$
\begin{aligned}
& \chi^{\mathcal{N}_{6 d}=(1,0)}(q ; \boldsymbol{y}, r) \\
& =\underbrace{\left(y_{1}^{2}+y_{1}^{-2}+y_{2}^{2}+y_{2}^{-2}+\frac{1}{2}[1]_{r} \prod_{i=1}^{2}\left(y_{i}+y_{i}^{-1}\right)\right) q^{0}}+\underbrace{\llbracket 1,0 ; 0 \rrbracket q}_{80 \text { states at level } 1} \\
& 8 \text { massless states } \\
& +\underbrace{(\llbracket 2,0 ; 0 \rrbracket+\llbracket 0,2 ; 0 \rrbracket+\llbracket 0,1 ; 1 \rrbracket) q^{2}}+(\llbracket 3,0 ; 0 \rrbracket+2 \llbracket 1,0 ; 0 \rrbracket+\llbracket 0,0 ; 0 \rrbracket \\
& 512 \text { states at level } 2 \\
& +\llbracket 1,2 ; 0 \rrbracket+\llbracket 0,2 ; 0 \rrbracket+\llbracket 0,0 ; 2 \rrbracket+2 \llbracket 1,1 ; 1 \rrbracket+\llbracket 0,1 ; 1 \rrbracket) q^{3}+\mathcal{O}\left(q^{4}\right) .
\end{aligned}
$$

The $q^{\leqslant 6}$ coefficients are listed in Table 4, further information on the particle content up to level 25 is tabulated in Appendix B.2.

\subsection{The total number of states at a given mass level}

In this subsection, we compute the total number of states present at a given mass level through the unrefined partition function, i.e. by setting the fugacities $y_{1}, y_{2}$ and $r$ in (5.4) to unity. The

\footnotetext{
23 Again, there is a subtlety in applying (5.3) to the massless R sector, see the footnote before (4.4). However, this can be fixed easily: one can simply add to it $\frac{1}{2}\left(y_{1}-y_{1}^{-1}\right)\left(y_{2}-y_{2}^{-1}\right)\left(r-r^{-1}\right)$ to get the correct massless character in $R$ sector.
} 
total number of states $N_{m}$ at the mass level $m$ can be read off from the coefficient of $q^{m}$ in the power series of $\chi^{\mathcal{N}_{6 d}=(1,0)}\left(q ;\left\{y_{i}=1, r=1\right\}\right)$.

We follow the analysis presented in Section 4.1. The unrefined partition function is given by

$$
\begin{aligned}
\chi^{\mathcal{N}_{6 d}=(1,0)}\left(q ;\left\{y_{i}=1, r=1\right\}\right) & =\left.2 \chi_{\mathrm{R}}^{\mathcal{N}_{6 d}=(1,0)}\right|_{\mathrm{GSO}}(q ; y=1, s=1) \\
& =\chi_{\mathrm{R}}^{S O(5)}\left(q ;\left\{y_{i}=1\right\}\right) \chi_{\mathrm{R}, h=1 / 4, \ell=1 / 2}^{\mathcal{N}_{2 d}=4, c=6}(q ; r=1) \\
& =q^{-1 / 8} \frac{\vartheta_{2}(1, q)^{4}}{\eta(q)^{9}}\left[1-2 i q^{1 / 8} \mu(1 / 2, \tau)\right] .
\end{aligned}
$$

Indeed, the power series of $\chi^{\mathcal{N}_{6 d}=(1,0)}\left(q ;\left\{y_{i}=1, r=1\right\}\right)$ in $q$ reproduces the numbers presented in the second column of Table 1 . Note that $\chi^{\mathcal{N}_{6 d}=(1,0)}\left(q ;\left\{y_{i}=1, r=1\right\}\right)$ is not a modular form, since the Appell-Lerch sum is a mock modular form and it is not added by a suitable nonholomorphic component to be modular.

\subsubsection{The number of states at each mass level and its asymptotics}

The number of states at the mass level $m$ can also be computed from

$$
N_{m}=\frac{1}{2 \pi i} \oint_{\mathcal{C}} \frac{\mathrm{d} q}{q^{m+1}} \chi^{\mathcal{N}_{6 d}=(1,0)}\left(q ;\left\{y_{i}=1, r=1\right\}\right),
$$

where $\mathcal{C}$ is a contour around the origin.

Now let us compute an asymptotic formula for the number of states $N_{m}$ at a mass level $m$ when $m \rightarrow \infty$. We focus on the limit $q \rightarrow 1^{-}$and proceed in a similar way to Section 4.1.

Let us first examine the leading behavior of $\mu(1 / 2, \tau)$ as $q \rightarrow 1^{-}$or $\tau \rightarrow 0$. Using the second point of Proposition 1.5 of [41], we find that

$$
\frac{1}{\sqrt{-i \tau}} \mu\left(\frac{1}{2 \tau},-\frac{1}{\tau}\right)+\mu\left(\frac{1}{2}, \tau\right)=\frac{1}{2 i} .
$$

Let us consider $\mu\left(\frac{1}{2 \tau},-\frac{1}{\tau}\right)$ as $q \rightarrow 1^{-}$or equivalently $\tau=i \epsilon$ as $\epsilon \rightarrow 0^{+}$. It follows from the definition of Appell-Lerch sum that

$$
\begin{aligned}
\mu\left(\frac{1}{2 \tau},-\frac{1}{\tau}\right) & =-\frac{e^{i \pi /(2 \tau)}}{\vartheta_{1}\left(e^{2 \pi i /(2 \tau)}, e^{-2 \pi i / \tau}\right)} \sum_{m \in \mathbb{Z}}(-1)^{m} \frac{e^{-i \pi m^{2} / \tau}}{1-e^{-2 \pi i m / \tau+\pi i / \tau}} \\
& \sim-\frac{e^{\pi /(2 \epsilon)}}{-i e^{\pi /(4 \epsilon)}} \times\left(-2 e^{-\pi / \epsilon}\right), \quad \tau=i \epsilon, \epsilon \rightarrow 0^{+} \\
& =2 i \exp \left(-\frac{3 \pi}{4 \epsilon}\right),
\end{aligned}
$$

where in the second 'equality' only $m=0,1$ in the infinite sum contribute to the leading behavior and we have used the fact that $\vartheta_{1}\left(e^{2 \pi i /(2 \tau)}, e^{-2 \pi i / \tau}\right)=-i e^{\pi /(4 \epsilon)}$, as $\tau=i \epsilon, \epsilon \rightarrow 0^{+}$. Hence, to the leading order, one can neglect the first term in (5.7) in comparison with $1 /(2 i)$ on the right-hand side and so

$$
\mu\left(\frac{1}{2}, \tau\right) \sim \frac{1}{2 i}, \quad q \rightarrow 1^{-} .
$$


Therefore it follows from (5.5) that, as $q \rightarrow 1^{-}$,

$$
\begin{aligned}
\chi^{\mathcal{N}_{6 d}=(1,0)}\left(q ;\left\{y_{i}=1, r=1\right\}\right) & \sim q^{-1 / 8} \frac{\vartheta_{2}(1, q)^{4}}{\eta(q)^{9}}\left(1-q^{1 / 8}\right) \\
& \sim(2 \pi)^{-5 / 2}\left(1-q^{1 / 8}\right)(1-q)^{5 / 2} \exp \left(-\frac{3 \pi^{2}}{2 \log q}\right),
\end{aligned}
$$

where we have used (4.13) and (4.11). Hence, as $m \rightarrow \infty$,

$$
N_{m} \sim(2 \pi)^{-5 / 2} \frac{1}{2 \pi i} \oint_{\mathcal{C}} \frac{\mathrm{d} q}{q}\left(1-q^{1 / 8}\right)(1-q)^{5 / 2} \exp \left(-\frac{3 \pi^{2}}{2 \log q}-m \log q\right) .
$$

The saddle point is at $q_{0}=\exp (-\pi \sqrt{3} / \sqrt{2 m})$ and the steepest descent direction is the imaginary direction in $q$. We proceed in a similar way to (4.17) by writing $q=q_{0} e^{i \theta}$ and using Laplace's method to obtain

$$
\begin{aligned}
N_{m} & \sim(2 \pi)^{-5 / 2}\left(1-q_{0}^{1 / 8}\right)\left(1-q_{0}\right)^{5 / 2} e^{\pi \sqrt{6 m}} \frac{1}{2 \pi} \int_{-\infty}^{\infty} \mathrm{d} \theta \exp \left(-\frac{1}{\pi} \sqrt{\frac{2}{3}} m^{3 / 2} \theta^{2}\right) \\
& \sim \frac{9 \pi}{2^{17 / 2}} m^{-5 / 2} \exp (\pi \sqrt{6 m}), \quad m \rightarrow \infty .
\end{aligned}
$$

\subsection{The GSO projected NS and R sectors}

\subsubsection{The NS sector}

From (6.50), the partition function of the GSO projected NS sector is

$$
\left.\chi_{\mathrm{NS}}^{\mathcal{N}_{6 d}=(1,0)}\right|_{\mathrm{GSO}}(q ; y, s)=\sum_{k_{1}, k_{2}, p=0}^{\infty}\left[2 k_{1}\right]_{y_{1}}\left[2 k_{2}\right]_{y_{2}}[2 p]_{r} F_{k_{1}, k_{2}, p}^{\mathrm{NS}}(q),
$$

where the function $F_{k, p}^{\mathrm{NS}}(q)$ is given by

$$
\begin{aligned}
& F_{k_{1}, k_{2}, p}^{\mathrm{NS}}(q) \\
& =(q ; q)_{\infty}^{-9}(1-q) q^{\frac{1}{2} p^{2}+p-1} \\
& \quad \times \sum_{\boldsymbol{n} \in \mathbb{Z}_{+}^{2}} \sum_{\boldsymbol{m} \in \mathbb{Z}_{\geqslant 0}^{2}} \prod_{A=1}^{2}(-1)^{n_{A}+1}\left(1-q^{n_{A}}\right) q^{\frac{1}{2} m_{A}^{2}+\left({ }^{n_{A}}\right)}\left(q^{n_{A}\left|k_{A}-m_{A}\right|}-q^{n_{A}\left(k_{A}+m_{A}+1\right)}\right) \\
& \quad \times \frac{1}{2}\left[\frac{\left(1-q^{p+\frac{1}{2}}\right) \vartheta_{3}(1, q)}{\left(1+q^{p-\frac{1}{2}}\right)\left(1+q^{p+\frac{3}{2}}\right)} \prod_{A=1}^{2}\left(1-q^{m_{A}+\frac{1}{2}}\right)\right. \\
& \left.\quad+(-1)^{m_{1}^{2}+m_{2}^{2}+p^{2}} \frac{\left(1+q^{p+\frac{1}{2}}\right) \vartheta_{4}(1, q)}{\left(1-q^{p-\frac{1}{2}}\right)\left(1-q^{p+\frac{3}{2}}\right)} \prod_{A=1}^{2}\left(1+q^{m_{A}+\frac{1}{2}}\right)\right]
\end{aligned}
$$

Asymptotics

This expression can be simplified further in the asymptotic limit $k_{1}, k_{2} \rightarrow \infty$. Using (4.20), we have 


$$
\begin{aligned}
& \sum_{\boldsymbol{n} \in \mathbb{Z}_{+}^{2}} \prod_{A=1}^{2}(-1)^{n_{A}+1}\left(1-q^{n_{A}}\right) q^{\left({ }^{n_{A}}\right)}\left(q^{n_{A}\left|k_{A}-m_{A}\right|}-q^{n_{A}\left(k_{A}+m_{A}+1\right)}\right) \\
& \sim(1-q)^{2} \prod_{A=1}^{2} \frac{q^{k_{A}}\left(1-q^{2 k_{A}+2}\right)}{\left(1+q^{k_{A}}\right)^{4}}\left\{q^{-m_{A}}\left(1-q^{2 m_{A}+1}\right)\right\},
\end{aligned}
$$

and using (4.22) we have

$$
\begin{aligned}
& \sum_{\boldsymbol{m} \in \mathbb{Z}_{\geqslant 0}^{2}} \prod_{A=1}^{2} q^{\frac{1}{2} m_{A}^{2}-m_{A}}\left(1-q^{m_{A}+\frac{1}{2}}\right)\left(1-q^{2 m_{A}+1}\right)=q^{-1}(1-q)^{2} \vartheta_{3}(1, q)^{2} \\
& \sum_{\boldsymbol{m} \in \mathbb{Z}_{\geqslant 0}^{2}} \prod_{A=1}^{2}(-1)^{m_{A}^{2}} q^{\frac{1}{2} m_{A}^{2}-m_{A}}\left(1+q^{m_{A}+\frac{1}{2}}\right)\left(1-q^{2 m_{A}+1}\right)=q^{-1}(1-q)^{2} \vartheta_{4}(1, q)^{2}
\end{aligned}
$$

Therefore we arrive at an asymptotic formula for $F_{k_{1}, k_{2}, p}^{\mathrm{NS}}(q)$ when $k_{1}, k_{2} \rightarrow \infty$ :

$$
\begin{aligned}
F_{k_{1}, k_{2}, p}^{\mathrm{NS}}(q) \sim & \frac{1}{2}(q ; q)_{\infty}^{-9}(1-q)^{5} q^{\frac{1}{2} p^{2}+p+k_{1}+k_{2}-2}\left[\frac{\left(1-q^{p+\frac{1}{2}}\right)}{\left(1+q^{p-\frac{1}{2}}\right)\left(1+q^{p+\frac{3}{2}}\right)} \vartheta_{3}(1, q)^{3}\right. \\
& \left.+(-1)^{p^{2}} \frac{\left(1+q^{p+\frac{1}{2}}\right)}{\left(1-q^{p-\frac{1}{2}}\right)\left(1-q^{p+\frac{3}{2}}\right)} \vartheta_{4}(1, q)^{3}\right], \quad k_{1}, k_{2} \rightarrow \infty
\end{aligned}
$$

The $R$ sector

The partition function of the GSO projected $\mathrm{R}$ sector is

$$
\left.\chi_{\mathrm{R}}^{\mathcal{N}_{6 d}=(1,0)}\right|_{\mathrm{GSO}}(q ; y, s)=\sum_{k_{1}, k_{2}, p=0}^{\infty}\left[2 k_{1}+1\right]_{y_{1}}\left[2 k_{2}+1\right]_{y_{2}}[2 p+1]_{r} F_{k_{1}, k_{2}, p}^{\mathrm{R}}(q),
$$

where $F_{k_{1}, k_{2}, p}^{\mathrm{R}}(q)$ is given by

$$
\begin{aligned}
& F_{k_{1}, k_{2}, p}^{\mathrm{R}}(q) \\
& =(q ; q)_{\infty}^{-9}(1-q) q^{\frac{1}{2} p^{2}+\frac{3}{2} p-\frac{3}{8}} \times \frac{1}{2} \frac{\left(1-q^{p+1}\right) \vartheta_{2}(1, q)}{\left(1+q^{p}\right)\left(1+q^{p+2}\right)} \\
& \quad \times \sum_{n \in \mathbb{Z}_{+}^{2}} \sum_{\boldsymbol{m} \in \mathbb{Z}_{\geqslant 0}^{2}} \prod_{A=1}^{2}(-1)^{n_{A}}\left(1-q^{n_{A}}\right) q^{\frac{1}{2}\left(m_{A}+\frac{1}{2}\right)^{2}+\left(\begin{array}{c}
n_{A} \\
2
\end{array}\right)\left(q^{n_{A}\left|k_{A}-m_{A}\right|}-q^{n_{A}\left(k_{A}+m_{A}+2\right)}\right)} \\
& \quad \times\left(1-q^{m_{A}+1}\right) .
\end{aligned}
$$

Similarly to the NS sector, an asymptotic formula for $F_{k_{1}, k_{2}, p}^{\mathrm{NS}}(q)$ when $k_{1}, k_{2} \rightarrow \infty$ is given by

$$
F_{k_{1}, k_{2}, p}^{\mathrm{R}}(q) \sim \frac{1}{2}(q ; q)_{\infty}^{-9}(1-q)^{5} q^{\frac{1}{2} p^{2}+\frac{3}{2} p+k_{1}+k_{2}-\frac{3}{8}} \frac{\left(1-q^{p+1}\right)}{\left(1+q^{p}\right)\left(1+q^{p+2}\right)} \vartheta_{2}(1, q)^{3}
$$


5.3. Multiplicities of representations in the $\mathcal{N}_{6 d}=(1,0)$ partition function

Combining the contributions from the NS and R sectors, we have

$$
\begin{aligned}
\chi^{\mathcal{N}_{6 d}=(1,0)}(q ; \boldsymbol{y}, r)= & \left.\chi_{\mathrm{NS}}^{\mathcal{N}_{6 d}=(1,0)}\right|_{\mathrm{GSO}}(q ; \boldsymbol{y}, r)+\left.\chi_{\mathrm{R}}^{\mathcal{N}_{6 d}=(1,0)}\right|_{\mathrm{GSO}}(q ; \boldsymbol{y}, r) \\
= & \sum_{k_{1}, k_{2}, p=0}^{\infty}\left(\left[2 k_{1}\right]_{y_{1}}\left[2 k_{2}\right]_{y_{2}}[2 p]_{r} F_{k_{1}, k_{2}, p}^{\mathrm{NS}}(q)\right. \\
& \left.+\left[2 k_{1}+1\right]_{y_{1}}\left[2 k_{2}+1\right]_{y_{2}}[2 p+1]_{r} F_{k_{1}, k_{2}, p}^{\mathrm{R}}(q)\right)
\end{aligned}
$$

Making SUSY manifest amounts to rewriting the partition function as

$$
\chi^{\mathcal{N}_{6 d}=(1,0)}(q ; \boldsymbol{y}, r)=\sum_{n_{1}, n_{2} \geqslant 0} \sum_{p=0}^{\infty} \llbracket n_{1}, n_{2} ; p \rrbracket G_{n_{1}, n_{2}, p}(q),
$$

and the aim is to compute explicitly a multiplicity generating function $G_{n_{1}, n_{2}, p}(q)$.

Before proceeding further, we observe the selection rule

$$
G_{n_{1}, 2 n_{2}, 2 p+1}(q)=0, \quad G_{n_{1}, 2 n_{2}+1,2 p}(q)=0 .
$$

It follows from (5.22) that $\left[k_{1}\right]_{y_{1}}\left[k_{2}\right]_{y_{2}}[p]_{r}$ with odd (respectively even) values of $p$ only enter with a product of two representations with both odd (resp. even) $k_{1}$ and $k_{2}$. According to (2.60) and (2.64), the product $\left[k_{1}\right]_{y_{1}}\left[k_{2}\right]_{y_{2}}$ with both odd (resp. even) $k_{1}$ and $k_{2}$ decomposes into only spin (resp. non-spin) representations of $S O(5)$. In other words, a spin (resp. non-spin) representation only comes with an odd (resp. even) value of $p$, and hence (5.24) follows.

The multiplicity of $\llbracket n_{1}, n_{2} ; p \rrbracket$ appearing in $\chi^{\mathcal{N}_{6 d}=(1,0)}(q ; \boldsymbol{y}, r)$ can be determined as follows:

$$
\begin{aligned}
G_{n_{1}, n_{2}, p}(q) & =\int \mathrm{d} \mu_{S U(2)}(r)[p]_{r} \int \mathrm{d} \mu_{S O(5)}(\boldsymbol{y})\left[n_{1}, n_{2}\right]_{y} \frac{\chi^{\mathcal{N}_{6 d}=(1,0)}(q ; \boldsymbol{y}, r)}{Z\left(\mathcal{N}_{6 d}=(1,0)\right)(\boldsymbol{y}, r)}, \\
& =G_{n_{1}, n_{2}, p}^{\mathrm{NS}}(q)+G_{n_{1}, n_{2}, p}^{\mathrm{R}}(q),
\end{aligned}
$$

where

$$
\begin{aligned}
G_{n_{1}, n_{2}, p}^{\mathrm{NS}}(q)= & \int \mathrm{d} \mu_{S U(2)}(r)[p]_{r} \int \mathrm{d} \mu_{S O(5)}(\boldsymbol{y})\left[n_{1}, n_{2}\right]_{\boldsymbol{y}} \\
& \times \sum_{k_{1}, k_{2}, p^{\prime} \geqslant 0} \frac{\left[2 k_{1}\right]_{y_{1}}\left[2 k_{2}\right]_{y_{2}}\left[2 p^{\prime}\right]_{r}}{Z\left(\mathcal{N}_{6 d}=(1,0)\right)(\boldsymbol{y}, r)} F_{k_{1}, k_{2}, p^{\prime}}^{\mathrm{NS}}(q), \\
G_{n_{1}, n_{2}, p}^{\mathrm{R}}(q)= & \int \mathrm{d} \mu_{S U(2)}(r)[p]_{r} \int \mathrm{d} \mu_{S O(5)}(\boldsymbol{y})\left[n_{1}, n_{2}\right]_{\boldsymbol{y}} \\
& \times \sum_{k_{1}, k_{2}, p^{\prime} \geqslant 0} \frac{\left[2 k_{1}+1\right]_{y_{1}}\left[2 k_{2}+1\right]_{y_{2}}\left[2 p^{\prime}+1\right]_{r}}{Z\left(\mathcal{N}_{6 d}=(1,0)\right)(\boldsymbol{y}, r)} F_{k_{1}, k_{2}, p^{\prime}}^{\mathrm{R}}(q),
\end{aligned}
$$

and the inverse of the character of the fundamental multiplet in (5.1) can be written as a geometric 
series $^{24}$ similar to $(4.42)$

$$
\begin{aligned}
{\left[Z\left(\mathcal{N}_{6 d}=(1,0)\right)(\boldsymbol{y}, r)\right]^{-1}=} & \frac{r^{2}}{\left(1+\frac{r}{y_{1} y_{2}}\right)\left(1+\frac{r y_{1}}{y_{2}}\right)\left(1+\frac{r y_{2}}{y_{1}}\right)\left(1+r y_{1} y_{2}\right)} \\
= & \sum_{m_{1}, \ldots, m_{4} \geqslant 0}(-1)^{m_{1}+m_{2}+m_{3}+m_{4}} r^{2+m_{1}+m_{2}+m_{3}+m_{4}} \\
& \times y_{1}^{-m_{1}+m_{2}-m_{3}+m_{4}} y_{2}^{-m_{1}-m_{2}+m_{3}+m_{4}} .
\end{aligned}
$$

\subsubsection{Some useful identities}

Before we proceed further, let us derive some useful identities for the elementary building blocks of $G_{n_{1}, n_{2}, p}$. The first one follows from (2.6):

$$
\begin{aligned}
\mathcal{I}_{0}\left(w ; p_{1}, p_{2}\right) & :=\int \mathrm{d} \mu_{S O(3)}(r) r^{w}\left[p_{1}\right]_{r}\left[p_{2}\right]_{r} \\
& = \begin{cases}\delta_{p_{1}, p_{2}} \\
\frac{1}{2} \sum_{p=0}^{\frac{1}{2}\left(p_{1}+p_{2}-\left|p_{1}-p_{2}\right|\right)}\left(\delta_{|w|, 2 p+\left|p_{1}-p_{2}\right|}-\delta_{|w|, 2 p+2+\left|p_{1}-p_{2}\right|}\right) & \text { for } w \neq 0 .\end{cases}
\end{aligned}
$$

Next, we are interested in the following integral:

$$
\mathcal{I}(\boldsymbol{w} ; \boldsymbol{k} ; \boldsymbol{n}):=\int \mathrm{d} \mu_{S O(5)}(\boldsymbol{y}) y_{1}^{w_{1}} y_{2}^{w_{2}}\left[k_{1}\right]_{y_{1}}\left[k_{2}\right]_{y_{2}}\left[n_{1}, n_{2}\right]_{\boldsymbol{y}} .
$$

There are four cases to be considered. Each of them can be computed using the decomposition formula (2.61) or (2.65), together with (5.30). In what follows, we assume that $\boldsymbol{k}, \boldsymbol{n} \in \mathbb{Z}_{\geqslant 0}^{2}$ and $\boldsymbol{w} \in \mathbb{Z}^{2}$.

$$
\begin{aligned}
& \mathcal{I}\left(\boldsymbol{w} ; 2 k_{1}, 2 k_{2} ; n_{1}, 2 n_{2}\right) \\
& \quad=\sum_{\boldsymbol{k}^{\prime} \in \mathbb{Z}_{\geqslant 0}^{2}} \Delta\left(n_{1}+n_{2}, n_{2} ; 2 k_{1}^{\prime}, 2 k_{2}^{\prime}\right) \prod_{A=1}^{2} \mathcal{I}_{0}\left(w_{A} ; 2 k_{A}, 2 k_{A}^{\prime}\right) \\
& \mathcal{I}\left(\boldsymbol{w} ; 2 k_{1}+1,2 k_{2}+1 ; n_{1}, 2 n_{2}\right) \\
& \quad=\sum_{\boldsymbol{k}^{\prime} \in \mathbb{Z}_{\geqslant 0}^{2}} \Delta\left(n_{1}+n_{2}, n_{2} ; 2 k_{1}^{\prime}, 2 k_{2}^{\prime}\right) \prod_{A=1}^{2} \mathcal{I}_{0}\left(w_{A} ; 2 k_{A}+1,2 k_{A}^{\prime}\right) \\
& \mathcal{I}\left(\boldsymbol{w} ; 2 k_{1}, 2 k_{2} ; n_{1}, 2 n_{2}+1\right) \\
& \quad=\sum_{\boldsymbol{k}^{\prime} \in \mathbb{Z}_{\geqslant 0}^{2}} \Delta\left(n_{1}+n_{2}+\frac{1}{2}, n_{2}+\frac{1}{2} ; 2 k_{1}^{\prime}+1,2 k_{2}^{\prime}+1\right) \prod_{A=1}^{2} \mathcal{I}_{0}\left(w_{A} ; 2 k_{A}, 2 k_{A}^{\prime}+1\right),
\end{aligned}
$$

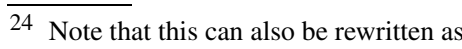

$$
\begin{aligned}
{\left[Z\left(\mathcal{N}_{6 d}=(1,0)\right)(\boldsymbol{y}, r)\right]^{-1} } & =r^{2} \operatorname{PE}\left[s[0,1]_{\boldsymbol{y}}\right] \quad \text { with } s=-r \\
& =\sum_{m=0}^{\infty}(-1)^{m} r^{m+2}[0, m] \boldsymbol{y}
\end{aligned}
$$


$\mathcal{I}\left(\boldsymbol{w} ; 2 k_{1}+1,2 k_{2}+1 ; n_{1}, 2 n_{2}+1\right)$

$$
=\sum_{\boldsymbol{k}^{\prime} \in \mathbb{Z}_{\geqslant 0}^{2}} \Delta\left(n_{1}+n_{2}+\frac{1}{2}, n_{2}+\frac{1}{2} ; 2 k_{1}^{\prime}+1,2 k_{2}^{\prime}+1\right) \prod_{A=1}^{2} \mathcal{I}_{0}\left(w_{A} ; 2 k_{A}+1,2 k_{A}^{\prime}+1\right),
$$

where from (2.57) and (2.62)

$$
\begin{aligned}
& \Delta\left(\lambda_{1}, \lambda_{2} ; 2 k_{1}, 2 k_{2}\right)=\frac{1}{2} \sum_{\sigma \in S_{2}} \operatorname{det}\left(\theta_{\left|\lambda_{A}-A+B\right|}^{4+\lambda_{A}-A-B}\left(k_{\sigma(A)}\right)\right)_{A, B=1}^{2}, \\
& \Delta\left(\lambda_{1}, \lambda_{2} ; 2 k_{1}+1,2 k_{2}+1\right)=\frac{1}{2} \sum_{\sigma \in S_{2}} \operatorname{det}\left(\theta_{\left|\lambda_{A}-A+B\right|}^{4+\lambda_{A}-A-B}\left(k_{\sigma(A)}+\frac{1}{2}\right)\right)_{A, B=1}^{2} .
\end{aligned}
$$

\subsubsection{Multiplicity generating function}

The NS and R sector contributions to the multiplicity generating function for the representation $\llbracket n_{1}, n_{2} ; p \rrbracket$ can be rewritten as

$$
\begin{aligned}
G_{n_{1}, n_{2}, p}^{\mathrm{NS}}(q)= & \sum_{m_{1}, \ldots, m_{4} \geqslant 0}(-1)^{\sum_{j=1}^{4} m_{j}} \sum_{p^{\prime} \geqslant 0} \mathcal{I}_{0}\left(W_{1}(\boldsymbol{m}), p, 2 p^{\prime}\right) \\
& \times \sum_{k_{1}, k_{2} \geqslant 0} \mathcal{I}\left(\boldsymbol{W}_{2}(\boldsymbol{m}) ; 2 k_{1}, 2 k_{2} ; n_{1}, n_{2}\right) F_{k_{1}, k_{2}, p^{\prime}}^{\mathrm{NS}}(q), \\
G_{n_{1}, n_{2}, p}^{\mathrm{R}}(q)= & \sum_{m_{1}, \ldots, m_{4} \geqslant 0}(-1)^{\sum_{j=1}^{4} m_{j}} \sum_{p^{\prime} \geqslant 0} \mathcal{I}_{0}\left(W_{1}(\boldsymbol{m}), p, 2 p^{\prime}+1\right) \\
& \times \sum_{k_{1}, k_{2} \geqslant 0} \mathcal{I}\left(\boldsymbol{W}_{2}(\boldsymbol{m}) ; 2 k_{1}+1,2 k_{2}+1 ; n_{1}, n_{2}\right) F_{k_{1}, k_{2}, p^{\prime}}^{\mathrm{R}}(q),
\end{aligned}
$$

where we define

$$
\begin{aligned}
& W_{1}(\boldsymbol{m})=2+m_{1}+m_{2}+m_{3}+m_{4}, \\
& \boldsymbol{W}_{2}(\boldsymbol{m})=\left(-m_{1}+m_{2}-m_{3}+m_{4},-m_{1}-m_{2}+m_{3}+m_{4}\right) .
\end{aligned}
$$

As stated in (5.25), the multiplicity of the representation $\llbracket n_{1}, n_{2} ; p \rrbracket$ in the $\mathcal{N}_{6 d}=(1,0)$ partition function is given by

$$
\begin{aligned}
G_{n_{1}, n_{2}, p}(q) & \\
= & G_{n_{1}, n_{2}, p}^{\mathrm{NS}}(q)+G_{n_{1}, n_{2}, p}^{\mathrm{R}}(q) \\
= & \sum_{m_{1}, \ldots, m_{4} \geqslant 0}(-1)^{\sum_{j=1}^{4} m_{j}} \sum_{p^{\prime} \geqslant 0}\left[\mathcal{I}_{0}\left(W_{1}(\boldsymbol{m}) ; p, 2 p^{\prime}\right)\right. \\
& \times \sum_{k_{1}, k_{2} \geqslant 0} \mathcal{I}\left(\boldsymbol{W}_{2}(\boldsymbol{m}) ; 2 k_{1}, 2 k_{2} ; n_{1}, n_{2}\right) F_{k_{1}, k_{2}, p^{\prime}}^{\mathrm{NS}}(q) \\
& \left.+\mathcal{I}_{0}\left(W_{1}(\boldsymbol{m}), p, 2 p^{\prime}+1\right) \sum_{k_{1}, k_{2} \geqslant 0} \mathcal{I}\left(\boldsymbol{W}_{2}(\boldsymbol{m}) ; 2 k_{1}+1,2 k_{2}+1 ; n_{1}, n_{2}\right) F_{k_{1}, k_{2}, p^{\prime}}^{\mathrm{R}}(q)\right] .
\end{aligned}
$$




\subsection{Empirical approach to $\mathcal{N}_{6 d}=(1,0)$ asymptotic patterns}

In this subsection, we follow the lines of Section 4.5 and investigate the large spin asymptotics of multiplicity generating functions $G_{n, k, p}(q)$ for universal $\mathcal{N}_{6 d}=(1,0)$ supermultiplets $\llbracket n, k ; p \rrbracket$. Similar to the $\mathcal{N}_{4 d}=1$ strategy, the $G_{n, k, p}(q)$ are expanded in powers of $q^{n}$ where $n$ denotes the first Dynkin label that we loosely identify with the spin. The coefficients $\tau_{\ell}^{k, p}(q)$ of $\left(q^{n}\right)^{\ell}$ turn out to be power series with non-negative coefficients which enter with alternating sign $(-1)^{\ell-1}$ :

$$
\begin{aligned}
G_{n, k, p}(q) & =q^{n} \tau_{1}^{k, p}(q)-q^{2 n} \tau_{2}^{k, p}(q)+q^{3 n} \tau_{3}^{k, p}(q)-\cdots \\
& =\sum_{\ell=1}^{\infty}(-1)^{\ell-1} q^{\ell n} \tau_{\ell}^{k, p}(q) .
\end{aligned}
$$

In spacetime dimensions higher than four, the analytic methods of Section 4.4 are no longer efficiently applicable. We could not find an asymptotic formula for (5.41) resembling (4.63) and (4.64) for the large spin regime of the $\mathcal{N}_{4 d}=1$ multiplicity generating functions. Hence, we determine the $\tau_{\ell}^{k, p}(q)$ including the leading trajectory $\tau_{1}^{k, p}(q)$ from our data found by expanding the partition function (5.3) up to mass level 25. The multiplicities of $\llbracket n, 0 ; 0 \rrbracket$ multiplets are shown in the following Table 5, data for non-zero values $(k, p)=(2,0),(0,2)$ and $(1,1)$ can be found in Appendix B.2. Table entries marked in red are only affected by the stable pattern $\tau_{\ell=1}^{k, p}(q)$ whereas the blue numbers arise from $q^{n} \tau_{1}^{k, p}(q)-q^{2 n} \tau_{2}^{k, p}(q)$, i.e. by including the (subtractive) subleading trajectory.

\subsubsection{Levels of first appearance}

Let us firstly determine the level of first appearance for various families $\{\llbracket n, k ; p \rrbracket, n=$ $0,1, \ldots\}$ of $\mathcal{N}_{6 d}=(1,0)$ supermultiplets with second $S O(5)$ Dynkin label $k$ and $R$ symmetry quantum number $p$ fixed. It is identical to the leading $q$ power of the multiplicity generating function $G_{0, k, p}(q)$ or its expansion coefficients $\tau_{\ell}^{k, p}(q)$ defined by (5.42). The following Table 6 gathers the mass levels $\alpha^{\prime} m^{2} \leqslant 25$ where the first instance of a $\{\llbracket n, k ; p \rrbracket, n=0,1, \ldots\}$ member can be found.

We observe that, roughly speaking, the level of first appearance for supermultiplets $\llbracket n, k ; p \rrbracket$ depends linearly ${ }^{25}$ on the $S O(5)$ Dynkin label $k$ (with slope $\frac{3}{2}$ ) but quadratically on the R symmetry spin $p / 2$, in agreement with the final remark in Section 3.2.

\subsubsection{Explicit formulae for the $\tau_{\ell}^{k, p}(q)$}

Let us now list the leading terms in $\tau_{\ell}^{0,0}(q), \tau_{\ell}^{2,0}(q), \tau_{\ell}^{0,2}(q)$ and $\tau_{\ell}^{1,1}(q)$, obtained through the entries of Table 5 and its $(k, p) \neq(0,0)$ relatives displayed in Appendix B.2. This allows to reconstruct the large spin asymptotics of the multiplicity generating functions $G_{n, k, p}(q)$ via (5.42).

\footnotetext{
25 The linear $k$ dependence can be partially understood from the $\lambda_{1,2}$ dependence in (2.68). However, the bosonic string suggests that an $S O(5)$ representation $[n, k]$ is delayed by two levels under $k \mapsto k+1$ whereas the observations from Table 6 clearly show a delay of three levels per $k \mapsto k+1$. Even though we cannot give a detailed explanation on analytical grounds, it is clear that this extra delay in mass level must be due to the worldsheet fermions, see e.g. (2.69) and (2.70).
} 
Table 5

$\mathcal{N}_{6 d}=(1,0)$ multiplets with $S O(5)$ quantum numbers $[n, 0]$ and $S U(2)_{R}$ spin 0.

\begin{tabular}{|c|c|c|c|c|c|c|c|c|c|c|c|c|}
\hline$\alpha^{\prime} m^{2}$ & $\# \llbracket 0,0 ; 0 \rrbracket$ & 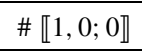 & $\# \llbracket 2,0 ; 0 \rrbracket$ & $\# \llbracket 3,0 ; 0 \rrbracket$ & $\# \llbracket 4,0 ; 0 \rrbracket$ & $\# \llbracket 5,0 ; 0 \rrbracket$ & $\# \llbracket 6,0 ; 0 \rrbracket$ & $\# \llbracket 7,0 ; 0 \rrbracket$ & 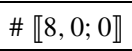 & 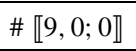 & $\# \llbracket 10,0 ; 0 \rrbracket$ & 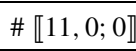 \\
\hline 1 & 0 & 1 & 0 & & & & & & & & & \\
\hline 2 & 0 & 0 & 1 & 0 & & & & & & & & \\
\hline 3 & 1 & 2 & 0 & 1 & 0 & & & & & & & \\
\hline 4 & 2 & 2 & 3 & 0 & 1 & 0 & & & & & & \\
\hline 5 & 3 & 9 & 4 & 3 & 0 & 1 & 0 & & & & & \\
\hline 6 & 8 & 12 & 14 & 5 & 3 & 0 & 1 & 0 & & & & \\
\hline 7 & 13 & 35 & 24 & 17 & 5 & 3 & 0 & 1 & 0 & & & \\
\hline 8 & 30 & 58 & 63 & 29 & 18 & 5 & 3 & 0 & 1 & 0 & & \\
\hline 9 & 53 & 135 & 116 & 82 & 32 & 18 & 5 & 3 & 0 & 1 & 0 & \\
\hline 10 & 107 & 243 & 265 & 153 & 88 & 33 & 18 & 5 & 3 & 0 & 1 & 0 \\
\hline 11 & 193 & 505 & 503 & 358 & 172 & 91 & 33 & 18 & 5 & 3 & 0 & 1 \\
\hline 12 & 376 & 918 & 1044 & 696 & 403 & 178 & 92 & 33 & 18 & 5 & 3 & 0 \\
\hline 13 & 670 & 1803 & 1975 & 1474 & 801 & 423 & 181 & 92 & 33 & 18 & 5 & 3 \\
\hline 14 & 1246 & 3269 & 3887 & 2839 & 1711 & 846 & 429 & 182 & 92 & 33 & 18 & 5 \\
\hline 15 & 2220 & 6136 & 7235 & 5687 & 3355 & 1824 & 866 & 432 & 182 & 92 & 33 & 18 \\
\hline 16 & 4005 & 11015 & 13691 & 10754 & 6784 & 3605 & 1870 & 872 & 433 & 182 & 92 & 33 \\
\hline 17 & 7025 & 20052 & 25041 & 20649 & 13021 & 7348 & 3718 & 1890 & 875 & 433 & 182 & 92 \\
\hline 18 & 12407 & 35469 & 45971 & 38304 & 25243 & 14213 & 7606 & 3764 & 1896 & 876 & 433 & 182 \\
\hline 19 & 21469 & 63030 & 82532 & 71226 & 47411 & 27774 & 14790 & 7720 & 3784 & 1899 & 876 & 433 \\
\hline 20 & 37182 & 109838 & 147906 & 129443 & 89013 & 52547 & 29015 & 15048 & 7766 & 3790 & 1900 & 876 \\
\hline 21 & 63492 & 191293 & 260818 & 234646 & 163536 & 99387 & 55177 & 29600 & 15162 & 7786 & 3793 & 1900 \\
\hline 22 & 108142 & 328527 & 457957 & 418298 & 299140 & 183903 & 104797 & 56431 & 29859 & 15208 & 7792 & 3794 \\
\hline 23 & 182254 & 562391 & 794256 & 741961 & 538495 & 338749 & 194850 & 107476 & 57016 & 29973 & 15228 & 7795 \\
\hline 24 & 306007 & 952431 & 1369976 & 1299438 & 963344 & 613928 & 360467 & 200360 & 108738 & 57275 & 30019 & 15234 \\
\hline 25 & 509309 & 1605996 & 2339762 & 2261945 & 1702039 & 1105604 & 656324 & 371692 & 203052 & 109324 & 57389 & 30039 \\
\hline
\end{tabular}


Table 6

Mass level where the $\llbracket 0, k ; p \rrbracket$ multiplet of $\mathcal{N}_{6 d}=(1,0)$ firstly occurs. Empty spaces indicate that the representations in question do not occur at levels $\leqslant 25$.

\begin{tabular}{|c|c|c|c|c|c|c|c|c|c|c|c|c|c|c|c|c|c|c|}
\hline$\downarrow p, \vec{k} !$ & 0 & 1 & 2 & 3 & 4 & 5 & 6 & 7 & 8 & 9 & 10 & 11 & 12 & 13 & 14 & 15 & 16 & 17 \\
\hline 0 & 1 & & 2 & & 5 & & 8 & & 11 & & 14 & & 17 & & 20 & & 23 & \\
\hline 1 & & 2 & & 4 & & 7 & & 10 & & 13 & & 16 & & 19 & & 22 & & 25 \\
\hline 2 & 3 & & 4 & & 7 & & 10 & & 13 & & 16 & & 19 & & 22 & & 25 & \\
\hline 3 & & 5 & & 7 & & 10 & & 13 & & 16 & & 19 & & 22 & & 25 & & \\
\hline 4 & 7 & & 8 & & 10 & & 13 & & 16 & & 19 & & 22 & & 25 & & & \\
\hline 5 & & 9 & & 11 & & 14 & & 17 & & 20 & & 23 & & & & & & \\
\hline 6 & 11 & & 12 & & 15 & & 18 & & 21 & & 24 & & & & & & & \\
\hline 7 & & 14 & & 16 & & 19 & & 22 & & 25 & & & & & & & & \\
\hline 8 & 17 & & 18 & & 20 & & 23 & & & & & & & & & & & \\
\hline 9 & & 20 & & 22 & & 25 & & & & & & & & & & & & \\
\hline 10 & 23 & & 24 & & & & & & & & & & & & & & & \\
\hline
\end{tabular}


- $S O(5)$ Dynkin labels $[n \rightarrow \infty, 0]$ and $S U(2)_{R}$ representation [0]

$$
\begin{aligned}
\tau_{1}^{0,0}(q)=1 & +0 q+3 q^{2}+5 q^{3}+18 q^{4}+33 q^{5}+92 q^{6}+182 q^{7}+433 q^{8} \\
& +876 q^{9}+1900 q^{10}+3794 q^{11}+7796 q^{12}+15238 q^{13} \\
& +30049 q^{14}+57465 q^{15}+109773 q^{16} \\
& +205349 q^{17}+382249 q^{18}+700520 q^{19}+\cdots, \\
\tau_{2}^{0,0}(q)= & q\left(1+4 q^{1}+10 q^{2}+30 q^{3}+76 q^{4}+190 q^{5}+449 q^{6}+1035 q^{7}+2298 q^{8}\right. \\
& \left.+4999 q^{9}+10580 q^{10}+21976 q^{11}+44727 q^{12}+89543 q^{13}+\cdots\right), \\
\tau_{3}^{0,0}(q)= & q\left(1+q+10 q^{2}+23 q^{3}+81 q^{4}+194 q^{5}+531 q^{6}+1232 q^{7}\right. \\
& \left.+2967 q^{8}+6586 q^{9}+\cdots\right), \\
\tau_{4}^{0,0}(q)= & q^{2}\left(1+5 q+16 q^{2}+53 q^{3}+153 q^{4}+417 q^{5}+\cdots\right), \\
\tau_{5}^{0,0}(q)= & q^{2}\left(1+q+11 q^{2}+\cdots\right) .
\end{aligned}
$$

- $S O(5)$ Dynkin labels $[n \rightarrow \infty, 2]$ and $S U(2)_{R}$ representation [0]

$$
\begin{aligned}
\tau_{1}^{2,0}(q)= & q^{2}\left(1+2 q+8 q^{2}+17 q^{3}+52 q^{4}+117 q^{5}+293 q^{6}+645 q^{7}+1468 q^{8}\right. \\
& +3119 q^{9}+6667 q^{10}+13674 q^{11}+27913 q^{12}+55446 q^{13}+109165 q^{14} \\
& \left.+210717 q^{15}+402714 q^{16}+757889 q^{17}+1412208 q^{18}+\cdots\right), \\
\tau_{2}^{2,0}(q)= & q^{3}\left(1+4 q+14 q^{2}+41 q^{3}+118 q^{4}+306 q^{5}+764 q^{6}+1818 q^{7}+4191 q^{8}\right. \\
& \left.+9344 q^{9}+20318 q^{10}+43083 q^{11}+89493 q^{12}+182239 q^{13}+\cdots\right), \\
\tau_{3}^{2,0}(q)= & q^{5}\left(3+9 q+40 q^{2}+114 q^{3}+345 q^{4}+890 q^{5}+2297 q^{6}+5481 q^{7}\right. \\
& \left.+12871 q^{8}+\cdots\right), \\
\tau_{4}^{2,0}(q)= & q^{6}\left(1+5 q+23 q^{2}+79 q^{3}+251 q^{4}+717 q^{5}+\cdots\right), \\
\tau_{5}^{2,0}(q)= & q^{8}\left(3+10 q+48 q^{2}+\cdots\right) .
\end{aligned}
$$

- $S O(5)$ Dynkin labels $[n \rightarrow \infty, 0]$ and $S U(2)_{R}$ representation [2]

$$
\begin{aligned}
\tau_{1}^{0,2}(q)= & q^{3}\left(3+5 q+20 q^{2}+46 q^{3}+128 q^{4}+288 q^{5}+696 q^{6}+1513 q^{7}+3354 q^{8}\right. \\
& +7025 q^{9}+14707 q^{10}+29736 q^{11}+59679 q^{12}+116933 q^{13} \\
& \left.+226900 q^{14}+432515 q^{15}+816089 q^{16}+\cdots\right), \\
\tau_{2}^{0,2}(q)= & q^{2}\left(1+3 q^{1}+13 q^{2}+37 q^{3}+109 q^{4}+285 q^{5}+727 q^{6}+1737 q^{7}\right. \\
& \left.+4050 q^{8}+9075 q^{9}+19868 q^{10}+42302 q^{11}+88278 q^{12}+\cdots\right), \\
\tau_{3}^{0,2}(q)= & q^{2}\left(1+2 q+13 q^{2}+37 q^{3}+124 q^{4}+331 q^{5}+906 q^{6}\right. \\
& \left.+2233 q^{7}+5456 q^{8}+\cdots\right), \\
\tau_{4}^{0,2}(q)= & q^{3}\left(2+7 q+29 q^{2}+92 q^{3}+282 q^{4}+\cdots\right), \\
\tau_{5}^{0,2}(q)= & q^{3}\left(1+3 q+18 q^{2}+\cdots\right) .
\end{aligned}
$$


- $S O(5)$ Dynkin labels $[n \rightarrow \infty, 1]$ and $S U(2)_{R}$ representation [1]

$$
\begin{aligned}
\tau_{1}^{1,1}(q)= & q^{2}\left(2+4 q+13 q^{2}+35 q^{3}+89 q^{4}+216 q^{5}+508 q^{6}+1145 q^{7}+2521 q^{8}\right. \\
& +5402 q^{9}+11320 q^{10}+23238 q^{11}+46856 q^{12}+92850 q^{13} \\
& \left.+181217 q^{14}+348612 q^{15}+661792 q^{16}+1240786 q^{17}+\cdots\right), \\
\tau_{2}^{1,1}(q)= & q^{2}\left(1+4 q+13 q^{2}+43 q^{3}+122 q^{4}+323 q^{5}+814 q^{6}+1962 q^{7}+4550 q^{8}\right. \\
& \left.+10233 q^{9}+22370 q^{10}+47718 q^{11}+99574 q^{12}+\cdots\right), \\
\tau_{3}^{1,1}(q)= & q^{3}\left(1+5 q+21 q^{2}+70 q^{3}+211 q^{4}+584 q^{5}+1529 q^{6}+3798 q^{7}+\cdots\right), \\
\tau_{4}^{1,1}(q)= & q^{4}\left(1+6 q+24 q^{2}+85 q^{3}+\cdots\right), \\
\tau_{5}^{1,1}(q)= & q^{5}(1+\cdots) .
\end{aligned}
$$

Further $\tau_{\ell}^{k, p}(q)$ are listed in AUXILIARY FILE 2. They suggest that the $\tau_{\ell}^{k, p}(q)$ expansion (5.42) converges more quickly with larger values of $k$ and smaller values of $p$.

\subsection{Four-dimensional $\mathcal{N}_{4 d}=2$ spectra}

In order to determine universal string spectra with $\mathcal{N}_{4 d}=2$ SUSY, we shall now compactify two dimensions of minimally supersymmetric $\mathcal{N}_{6 d}=(1,0)$ theories on a $T^{2}$. This preserves all the eight supercharges and the internal rotation symmetry becomes an $R$ symmetry factor of $S O(2)_{R} \cong U(1)_{R}$. Hence, the dimensionally reduced theory in $d=4$ spacetime dimensions enjoys $\mathcal{N}_{4 d}=2$ SUSY and R symmetry $S U(2)_{R} \times U(1)_{R}$. The fundamental $\mathcal{N}_{4 d}=2$ superPoincaré multiplet encompasses $8+8$ states,

$$
Z\left(\mathcal{N}_{4 d}=2\right)=[2]_{y}+[2]_{r}[0]_{y}+\left(z^{2}+z^{-2}\right)[0]_{y}+\left(z+z^{-1}\right)[1]_{r}[1]_{y}
$$

where $z$ denotes the $U(1)_{R}$ fugacity. The tensor product of (5.47) with a Clifford vacuum in some $S O(3) \times S U(2)_{R} \times U(1)_{R}$ representation yields a family of supermultiplets characterized by three quantum numbers $-n$ for $S O(3)$ spin, $m$ for $S U(2)_{R}$ spin and $p$ for $U(1)_{R}$ charge. The resulting $16(n+1)(m+1)$ states are described by the supercharacter ${ }^{26}$

26 The simplicity of the $S O(3)$ tensor product $[2 m] \cdot[2 k]=\sum_{l=|k-m|}^{k+m}[2 l]$ allows for compact closed formulae for the $S O(3) \times S U(2)_{R} \times U(1)_{R}$ decomposition of a general $\mathcal{N}_{4 d}=2$ supercharacter:

$$
\begin{aligned}
\llbracket n ; m, p \rrbracket= & z^{p}\left\{[m]_{r}[n+2]+[m]_{r}[n-2]+[m+2]_{r}[n]+[m-2]_{r}[n]+2[m]_{r}[n]\right. \\
& \left.+\left(z^{2}+z^{-2}\right)[m]_{r}[n]+\left(z+z^{-1}\right)\left([m+1]_{r}+[m-1]_{r}\right)([n+1]+[n-1])\right\} .
\end{aligned}
$$

This generic character formula (5.48) holds for values $n, m \geqslant 2$ of the Clifford vacuum's $S O(3) \times S U(2)_{R}$ spin quantum numbers and specializes otherwise:

$$
\begin{aligned}
\llbracket n ; 0, p \rrbracket= & z^{p}\left\{[n+2]+[n-2]+[2]_{r}[n]+\left(1+z^{2}+z^{-2}\right)[n]\right. \\
& \left.+\left(z+z^{-1}\right)[1]_{r}([n+1]+[n-1])\right\}, \quad n \geqslant 2, \\
\llbracket 0 ; m, p \rrbracket= & z^{p}\left\{[m]_{r}[2]+[m]_{r}[0]+[m+2]_{r}[0]+[m-2]_{r}[0]+\left(z^{2}+z^{-2}\right)[m]_{r}[0]\right. \\
& \left.+\left(z+z^{-1}\right)\left([m+1]_{r}+[m-1]_{r}\right)[1]\right\}, \quad m \geqslant 2, \\
\llbracket 0 ; 0, p \rrbracket= & z^{p}\left\{[2]+[2]_{r}[0]+\left(z^{2}+z^{-2}\right)[0]+\left(z+z^{-1}\right)[1]_{r}[1]\right\},
\end{aligned}
$$




$$
\llbracket n ; m, p \rrbracket:=Z\left(\mathcal{N}_{4 d}=2\right) \cdot z^{p}[m]_{r}[n]_{y} .
$$

The position of the semicolon in the arguments of the supercharacter allows to distinguish $\mathcal{N}_{4 d}=$ 2 multiplets $\llbracket \cdot ; \cdot, \cdot \rrbracket$ from $\mathcal{N}_{6 d}=(1,0)$ multiplets $\llbracket \cdot, \cdot ; \cdot \rrbracket$.

The universal partition function of $\mathcal{N}_{4 d}=2$ scenarios is obtained through GSO projection of the following character products:

$$
\begin{aligned}
\chi^{\mathcal{N}_{4 d}=2}(q ; y, r, z)= & \left.\chi_{\mathrm{NS}}^{\mathcal{N}_{4 d}=2}\right|_{\mathrm{GSO}}(q ; y, r, z)+\left.\chi_{\mathrm{R}}^{\mathcal{N}_{4 d}=2}\right|_{\mathrm{GSO}}(q ; y, r, z), \\
\left.\chi_{\mathrm{NS}}^{\mathcal{N}_{4 d}=2}\right|_{\mathrm{GSO}}(q ; y, r, z)= & \frac{1}{2} q^{-\frac{1}{2}}\left[\chi_{\mathrm{NS}}^{S O(3)}(q ; y) \chi_{\mathrm{NS}, h=0, \ell=0}^{\mathcal{N}_{22}=4, c=6}(q ; r) \chi_{\mathrm{NS}}^{S O(3)}(q ; z)\right. \\
& \left.-\chi_{\mathrm{NS}}^{S O(3)}\left(e^{2 \pi i} q ; y\right) \chi_{\mathrm{NS}, h=0, \ell=0}^{\mathcal{N}_{2 d}=4, c=6}\left(e^{2 \pi i} q ; r\right) \chi_{\mathrm{NS}}^{S O(3)}\left(e^{2 \pi i} q ; z\right)\right], \\
\left.\chi_{\mathrm{R}}^{\mathcal{N}_{4 d}=2}\right|_{\mathrm{GSO}}(q ; y, r, z)= & \frac{1}{2} \chi_{\mathrm{R}}^{S O(3)}(q ; y) \chi_{\mathrm{R}, h=1 / 4, \ell=1 / 2}^{\mathcal{N}_{2 d}=4, c=6}(q ; r) \chi_{\mathrm{R}}^{S O(3)}(q ; z) .
\end{aligned}
$$

Its symmetry under reversal $p \mapsto-p$ of $U(1)_{R}$ charges motivates the definition

$$
\llbracket n ; m, \pm p \rrbracket:= \begin{cases}\llbracket n ; m, p \rrbracket+\llbracket n ; m,-p \rrbracket, & p \neq 0, \\ \llbracket n ; m, 0 \rrbracket, & p=0,\end{cases}
$$

then the power series expansion of (5.54) starts like ${ }^{27}$

$$
\begin{aligned}
\chi^{\mathcal{N}_{4 d}}= & 2(q ; y, r, z) \\
= & \underbrace{\left(y^{2}+y^{-2}+z^{2}+z^{-2}+\frac{1}{2}\left(y+y^{-1}\right)[1]_{z}[1] r\right) q^{0}}_{8 \text { massless states }} \\
& +\underbrace{(\llbracket 2 ; 0,0 \rrbracket+\llbracket 0 ; 0, \pm 2 \rrbracket) q}_{80 \text { states at level } 1} \\
& +\underbrace{(\llbracket 4 ; 0,0 \rrbracket+2 \llbracket 2 ; 0, \pm 2 \rrbracket+\llbracket 2 ; 0,0 \rrbracket+\llbracket 1 ; 1, \pm 1 \rrbracket+\llbracket 0 ; 0, \pm 4 \rrbracket+2 \llbracket 0 ; 0,0 \rrbracket) q^{2}}_{512 \text { states at level } 2} \\
& +(\llbracket 6 ; 0,0 \rrbracket+2 \llbracket 4 ; 0, \pm 2 \rrbracket+\llbracket 4 ; 0,0 \rrbracket+2 \llbracket 3 ; 1, \pm 1 \rrbracket+2 \llbracket 2 ; 0, \pm 4 \rrbracket \\
& +2 \llbracket 2 ; 0, \pm 2 \rrbracket+6 \llbracket 2 ; 0,0 \rrbracket+2 \llbracket 1 ; 1, \pm 3 \rrbracket+3 \llbracket 1 ; 1, \pm 1 \rrbracket+\llbracket 0 ; 2,0 \rrbracket+\llbracket 0 ; 0, \pm 6 \rrbracket \\
& +4 \llbracket 0 ; 0, \pm 2 \rrbracket+2 \llbracket 0 ; 0,0 \rrbracket) q^{3}+\mathcal{O}\left(q^{4}\right) .
\end{aligned}
$$

The vertex operators occurring in the three multiplets of the first mass level have been constructed in [33], see Eqs. (6.3) to (6.11) of that reference for bosons and Eqs. (6.22) to (6.30) for fermions. The content of the first five levels is summarized in Table 7.

Comparison with the partition function (5.4) of the $\mathcal{N}_{6 d}=(1,0)$ ancestor theory (and Table 4) clearly demonstrates that the six-dimensional viewpoint gives a more streamlined handle on the

$$
\begin{aligned}
\llbracket 1 ; 1, p \rrbracket= & z^{p}\left\{[1]_{r}[3]+[3]_{r}[1]+\left(2+z^{2}+z^{-2}\right)[1]_{r}[1]\right. \\
& \left.+\left(z+z^{-1}\right)\left([2]_{r}[2]+[2]_{r}[0]+[2]+[0]\right)\right\} .
\end{aligned}
$$

We observe the general selection rule that either none or all of $n, m, p$ are odd, hence, there is no need to consider $\llbracket 1 ; 0, p \rrbracket$ or $\llbracket 0 ; 1, p \rrbracket$.

27 Again, there is a subtlety in applying the above formula to the massless R sector; see the footnote before (4.4). However, this can be fixed easily: one can simply add to it $\frac{1}{2}\left(y-y^{-1}\right)\left(z-z^{-1}\right)\left(r-r^{-1}\right)$ to get the correct massless character in $\mathrm{R}$ sector. 
Table 7

$\mathcal{N}_{4 d}=2$ multiplets occurring up to mass level 5.

\begin{tabular}{|c|c|}
\hline$\alpha^{\prime} m^{2}$ & Representations of $\mathcal{N}_{4 d}=2$ super-Poincaré \\
\hline 1 & $\llbracket 2 ; 0,0 \rrbracket+\llbracket 0 ; 0, \pm 2 \rrbracket$ \\
\hline 2 & $\llbracket 4 ; 0,0 \rrbracket+2 \llbracket 2 ; 0, \pm 2 \rrbracket+\llbracket 2 ; 0,0 \rrbracket+\llbracket 1 ; 1, \pm 1 \rrbracket+\llbracket 0 ; 0, \pm 4 \rrbracket+2 \llbracket 0 ; 0,0 \rrbracket$ \\
\hline 3 & $\begin{array}{l}\llbracket 6 ; 0,0 \rrbracket+2 \llbracket 4 ; 0, \pm 2 \rrbracket+\llbracket 4 ; 0,0 \rrbracket+2 \llbracket 3 ; 1, \pm 1 \rrbracket+2 \llbracket 2 ; 0, \pm 4 \rrbracket+2 \llbracket 2 ; 0, \pm 2 \rrbracket+6 \llbracket 2 ; 0,0 \rrbracket \\
\quad+2 \llbracket 1 ; 1, \pm 3 \rrbracket+3 \llbracket 1 ; 1, \pm 1 \rrbracket+\llbracket 0 ; 2,0 \rrbracket+\llbracket 0 ; 0, \pm 6 \rrbracket+4 \llbracket 0 ; 0, \pm 2 \rrbracket+2 \llbracket 0 ; 0,0 \rrbracket\end{array}$ \\
\hline 4 & $\begin{array}{l}\llbracket 8 ; 0,0 \rrbracket+2 \llbracket 6 ; 0, \pm 2 \rrbracket+\llbracket 6 ; 0,0 \rrbracket+2 \llbracket 5 ; 1, \pm 1 \rrbracket+2 \llbracket 4 ; 0, \pm 4 \rrbracket+3 \llbracket 4 ; 0, \pm 2 \rrbracket+8 \llbracket 4 ; 0,0 \rrbracket \\
\quad+3 \llbracket 3 ; 1, \pm 3 \rrbracket+6 \llbracket 3 ; 1, \pm 1 \rrbracket+\llbracket 2 ; 2, \pm 2 \rrbracket+3 \llbracket 2 ; 2,0 \rrbracket+2 \llbracket 2 ; 0, \pm 6 \rrbracket+3 \llbracket 2 ; 0, \pm 4 \rrbracket+12 \llbracket 2 ; 0, \pm 2 \rrbracket \\
\quad+11 \llbracket 2 ; 0,0 \rrbracket+2 \llbracket 1 ; 1, \pm 5 \rrbracket+5 \llbracket 1 ; 1, \pm 3 \rrbracket+10 \llbracket 1 ; 1, \pm 1 \rrbracket+2 \llbracket 0 ; 2, \pm 2 \rrbracket+\llbracket 0 ; 2,0 \rrbracket+\llbracket 0 ; 0, \pm 8 \rrbracket \\
\quad+5 \llbracket 0 ; 0, \pm 4 \rrbracket+4 \llbracket 0 ; 0,2 \rrbracket+11 \llbracket 0 ; 0,0 \rrbracket\end{array}$ \\
\hline 5 & $\begin{array}{l}\llbracket 10 ; 0,0 \rrbracket+2 \llbracket 8 ; 0, \pm 2 \rrbracket+\llbracket 8 ; 0,0 \rrbracket+2 \llbracket 7 ; 1, \pm 1 \rrbracket+2 \llbracket 6 ; 0, \pm 4 \rrbracket+3 \llbracket 6 ; 0, \pm 2 \rrbracket+8 \llbracket 6 ; 0,0 \rrbracket \\
\quad+3 \llbracket 5 ; 1, \pm 3 \rrbracket+7 \llbracket 5 ; 1, \pm 1 \rrbracket+\llbracket 4 ; 2, \pm 2 \rrbracket+4 \llbracket 4 ; 2,0 \rrbracket+2 \llbracket 4 ; 0, \pm 6 \rrbracket+4 \llbracket 4 ; 0, \pm 4 \rrbracket+16 \llbracket 4 ; 0, \pm 2 \rrbracket \\
\quad+17 \llbracket 4 ; 0,0 \rrbracket+3 \llbracket 3 ; 1, \pm 5 \rrbracket+11 \llbracket 3 ; 1, \pm 3 \rrbracket+21 \llbracket 3 ; 1, \pm 1 \rrbracket+\llbracket 2 ; 2, \pm 4 \rrbracket+7 \llbracket 2 ; 2, \pm 2 \rrbracket+8 \llbracket 2 ; 2,0 \rrbracket \\
\quad+2 \llbracket 2 ; 0, \pm 8 \rrbracket+3 \llbracket 2 ; 0, \pm 6 \rrbracket+15 \llbracket 2 ; 0, \pm 4 \rrbracket+23 \llbracket 2 ; 0, \pm 2 \rrbracket+38 \llbracket 2 ; 0,0 \rrbracket+\llbracket 1 ; 3, \pm 1 \rrbracket+2 \llbracket 1 ; 1, \pm 7 \rrbracket \\
\quad+6 \llbracket 1 ; 1, \pm 5 \rrbracket+16 \llbracket 1 ; 1, \pm 3 \rrbracket+28 \llbracket 1 ; 1, \pm 1 \rrbracket+3 \llbracket 0 ; 2, \pm 4 \rrbracket+4 \llbracket 0 ; 2, \pm 2 \rrbracket+9 \llbracket 0 ; 2,0 \rrbracket+\llbracket 0 ; 0, \pm 10 \rrbracket \\
\quad+5 \llbracket 0 ; 0, \pm 6 \rrbracket+6 \llbracket 0 ; 0, \pm 4 \rrbracket+21 \llbracket 0 ; 0, \pm 2 \rrbracket+16 \llbracket 0 ; 0,0 \rrbracket\end{array}$ \\
\hline
\end{tabular}

spectrum in terms of fewer supermultiplets. This is why we do not provide an asymptotic analysis and data tables for the universal $\mathcal{N}_{4 d}=2$ spectrum like we did for the $d=6$ ancestor in Section 5.4 and Appendix B.2.

\section{Spectra in compactifications with 16 supercharges}

This section is devoted to maximally supersymmetric Type I superstring compactifications on even-dimensional tori where all the sixteen supercharges are preserved [1]. The methods introduced in Sections 2.4 and 2.5 are applied to decompose the partition function of the $\left(\partial X^{i}, \psi^{i}\right)$ CFT describing $d=10,8,6,4$ spacetime dimensions into characters of the little group $S O(d-1)$. According to Fig. 1 , the $d=10$ case takes the role of the ancestor theory for 16 supercharges, so its spectrum will be analyzed in particular detail. In the remaining cases $d=8,6,4$, dimensional reduction converts part of the higher-dimensional Lorentz symmetry into an internal $R$ symmetry, i.e. we branch the ten-dimensional little group into $S O(9) \rightarrow S O(d-1) \times S O(10-d)_{R}$. In this process, individual Lorentz fugacities $y_{k}$ with $k>\frac{1}{2}(d-2)$ are reinterpreted as R symmetry fugacities $r_{k}$.

Before looking at individual dimensionalities in detail, let us fix the notation for describing supersymmetric spectra with R symmetries: Characters of the spacetime little group $S O(d-1)$ are denoted by $\left[a_{1}, \ldots, a_{n}\right]$ with fugacities $y_{1}, \ldots, y_{n}$ and $n=\frac{1}{2}(d-2)$ whereas those of the R symmetry $S O(10-d)_{R}$ receive an extra subscript $\left[b_{1}, \ldots, b_{\ell}\right]_{R}$ with fugacities $r_{1}, \ldots, r_{\ell}$ and $\ell=5-\frac{d}{2}$. Our notation for supercharacters makes use of double brackets $\llbracket a_{1}, \ldots, a_{n} ; b_{1}, \ldots, b_{\ell} \rrbracket$ enclosing the $S O(d-1) \times S O(10-d)_{R}$ quantum numbers of the highest weight state. The semicolon between $a_{n}$ and $b_{1}$ separates spacetime from R symmetry Dynkin labels and eliminates any ambiguity about the spacetime dimension under consideration.

\subsection{Ten-dimensional $\mathcal{N}_{10 d}=1$ spectra}

In this subsection, we want to revisit the results of [9] on $S O(9)$ covariant partition functions for ten-dimensional open string excitations and examine further symmetry patterns. The minimal 
massive $\mathcal{N}_{10 d}=1$ SUSY multiplet encompasses $S O(9)$ representations of a spin two tensor, a three-form and a massive gravitino ${ }^{28}$

$$
Z\left(\mathcal{N}_{10 d}=1\right):=[2,0,0,0]+[0,0,1,0]+[1,0,0,1]
$$

This is precisely the particle content of the first mass level, its vertex operators can for instance be found in Eqs. (2.8), (2.9) and (2.22) of [33].

The generic multiplet is obtained as a tensor product of $Z\left(\mathcal{N}_{10 d}=1\right)$ with some $S O(9)$ representation and therefore described by the following $\mathcal{N}_{10 d}=1$ supercharacter:

$$
\llbracket a_{1}, a_{2}, a_{3}, a_{4} \rrbracket:=Z\left(\mathcal{N}_{10 d}=1\right) \cdot\left[a_{1}, a_{2}, a_{3}, a_{4}\right] .
$$

This is the basic building blocks of the refined ten-dimensional partition function. The latter can be obtained through standard GSO projection of the spacetime CFT

$$
\begin{aligned}
& \chi^{\mathcal{N}_{10 d}=1}(q ; \boldsymbol{y})=\left.\chi_{\mathrm{NS}}^{\mathcal{N}_{10 d}=1}\right|_{\mathrm{GSO}}(q ; \boldsymbol{y})+\left.\chi_{\mathrm{R}}^{\mathcal{N}_{10 d}=1}\right|_{\mathrm{GSO}}(q ; \boldsymbol{y}), \\
& \left.\chi_{\mathrm{NS}}^{\mathcal{N}_{10 d}=1}\right|_{\mathrm{GSO}}(q ; \boldsymbol{y}, r)=\frac{1}{2} q^{-\frac{1}{2}}\left[\chi_{\mathrm{NS}}^{S O(9)}(q ; \boldsymbol{y})-\chi_{\mathrm{NS}}^{S O(9)}\left(e^{2 \pi i} q ; \boldsymbol{y}\right)\right], \\
& \left.\chi_{\mathrm{R}}^{\mathcal{N}_{10 d}=1}\right|_{\mathrm{GSO}}(q ; \boldsymbol{y}, r)=\frac{1}{2} \chi_{\mathrm{R}}^{\operatorname{SO}(9)}(q ; \boldsymbol{y}),
\end{aligned}
$$

where $\chi_{\mathrm{NS}}^{S O(9)}(q ; \boldsymbol{y})$ and $\chi_{\mathrm{R}}^{S O(9)}(q ; \boldsymbol{y})$ are given by $(2.69)$ and $(2.70)$.

In a power series expansion in $q$, the coefficient of the $n$th power $q^{n}$ comprises the superPoincaré characters of the $n$th mass level $m^{2}=n / \alpha^{\prime 29}$ :

$$
\begin{aligned}
\chi^{\mathcal{N}_{10 d}}=1 & (q ; \boldsymbol{y}) \\
= & \underbrace{\left(\sum_{j=1}^{4}\left(y_{j}^{2}+y_{j}^{-2}\right)+\frac{1}{2} \prod_{j=1}^{4}\left(y_{j}+y_{j}^{-1}\right)\right) q^{0}}_{16 \text { massless states }} \\
& +\underbrace{\llbracket 0,0,0,0 \rrbracket q}_{256 \text { states at level } 1} \\
& +\underbrace{\llbracket 1,0,0,0 \rrbracket q^{2}}_{2304 \text { states at level } 2}+\underbrace{(\llbracket 2,0,0,0 \rrbracket+\llbracket 0,0,0,1 \rrbracket) q^{3}}_{15360 \text { states at level } 3} \\
& +(\llbracket 3,0,0,0 \rrbracket+\llbracket 1,0,0,1 \rrbracket+\llbracket 1,0,0,0 \rrbracket+\llbracket 0,1,0,0 \rrbracket) q^{4} \\
& +\mathcal{O}\left(q^{5}\right) .
\end{aligned}
$$

The supermultiplets up to level eight are listed in Table 8 and the complete first 25 mass levels can be found in Table 9 and Appendix B.3.

\footnotetext{
28 Note that $Z\left(\mathcal{N}_{10 d}=1\right)$ is denoted by $Z_{Q}$ in [9].

29 Note the usual subtlety about the massless R sector which was explained in the footnote before (4.4). One can simply fix this by adding $\frac{1}{2}\left([0,0,0,1]_{S O(8)}-[0,0,1,0]_{S O(8)}\right)=\frac{1}{2} \prod_{i=1}^{4}\left(y_{i}-y_{i}^{-1}\right)$ to the present result and obtain the correct answer; see also (3.16) of [9]. The $\frac{1}{2}[1,0,0,0]_{9}$ factor in the massive sector of the aforementioned (3.16) exactly matches our formula at any positive $q$ power.
} 
Table 8

$\mathcal{N}_{10 d}=1$ multiplets occurring up to mass level eight.

\begin{tabular}{|c|c|}
\hline$\alpha^{\prime} m^{2}$ & Representations of $\mathcal{N}_{10 d}=1$ super-Poincaré \\
\hline 1 & $\llbracket 0,0,0,0 \rrbracket$ \\
\hline 2 & $\llbracket 1,0,0,0 \rrbracket$ \\
\hline 3 & $\llbracket 2,0,0,0 \rrbracket+\llbracket 0,0,0,1 \rrbracket$ \\
\hline 4 & $\llbracket 3,0,0,0 \rrbracket+\llbracket 1,0,0,1 \rrbracket+\llbracket 1,0,0,0 \rrbracket+\llbracket 0,1,0,0 \rrbracket$ \\
\hline 5 & $\begin{array}{l}\llbracket 4,0,0,0 \rrbracket+\llbracket 2,0,0,1 \rrbracket+\llbracket 2,0,0,0 \rrbracket+\llbracket 1,1,0,0 \rrbracket+\llbracket 1,0,0,1 \rrbracket+\llbracket 0,1,0,0 \rrbracket+\llbracket 0,0,1,0 \rrbracket \\
\quad+\llbracket 0,0,0,1 \rrbracket+\llbracket 0,0,0,0 \rrbracket\end{array}$ \\
\hline 6 & $\begin{array}{l}\llbracket 5,0,0,0 \rrbracket+\llbracket 3,0,0,1 \rrbracket+\llbracket 3,0,0,0 \rrbracket+\llbracket 2,1,0,0 \rrbracket+\llbracket 2,0,0,1 \rrbracket+\llbracket 2,0,0,0 \rrbracket+2 \llbracket 1,1,0,0 \rrbracket \\
\quad+\llbracket 1,0,1,0 \rrbracket+2 \llbracket 1,0,0,1 \rrbracket+2 \llbracket 1,0,0,0 \rrbracket+\llbracket 0,1,0,1 \rrbracket+\llbracket 0,1,0,0 \rrbracket+\llbracket 0,0,0,2 \rrbracket+2 \llbracket 0,0,0,1 \rrbracket\end{array}$ \\
\hline 7 & $\begin{array}{l}\llbracket 6,0,0,0 \rrbracket+\llbracket 4,0,0,1 \rrbracket+\llbracket 4,0,0,0 \rrbracket+\llbracket 3,1,0,0 \rrbracket+\llbracket 3,0,0,1 \rrbracket+\llbracket 3,0,0,0 \rrbracket+2 \llbracket 2,1,0,0 \rrbracket \\
\quad+\llbracket 2,0,1,0 \rrbracket+3 \llbracket 2,0,0,1 \rrbracket+3 \llbracket 2,0,0,0 \rrbracket+\llbracket 1,1,0,1 \rrbracket+2 \llbracket 1,1,0,0 \rrbracket+\llbracket 1,0,1,0 \rrbracket+\llbracket 1,0,0,2 \rrbracket \\
\quad+4 \llbracket 1,0,0,1 \rrbracket+2 \llbracket 1,0,0,0 \rrbracket+\llbracket 0,2,0,0 \rrbracket+2 \llbracket 0,1,0,1 \rrbracket+2 \llbracket 0,1,0,0 \rrbracket+3 \llbracket 0,0,1,0 \rrbracket \\
\quad+\llbracket 0,0,0,2 \rrbracket+2 \llbracket 0,0,0,1 \rrbracket+2 \llbracket 0,0,0,0 \rrbracket\end{array}$ \\
\hline 8 & $\begin{array}{l}\llbracket 7,0,0,0 \rrbracket+\llbracket 5,0,0,1 \rrbracket+\llbracket 5,0,0,0 \rrbracket+\llbracket 4,1,0,0 \rrbracket+\llbracket 4,0,0,1 \rrbracket+\llbracket 4,0,0,0 \rrbracket+2 \llbracket 3,1,0,0 \rrbracket \\
\quad+\llbracket 3,0,1,0 \rrbracket+3 \llbracket 3,0,0,1 \rrbracket+4 \llbracket 3,0,0,0 \rrbracket+\llbracket 2,1,0,1 \rrbracket+3 \llbracket 2,1,0,0 \rrbracket+\llbracket 2,0,1,0 \rrbracket+\llbracket 2,0,0,2 \rrbracket \\
\quad+5 \llbracket 2,0,0,1 \rrbracket+3 \llbracket 2,0,0,0 \rrbracket+\llbracket 1,2,0,0 \rrbracket+3 \llbracket 1,1,0,1 \rrbracket+5 \llbracket 1,1,0,0 \rrbracket+4 \llbracket 1,0,1,0 \rrbracket \\
\quad+2 \llbracket 1,0,0,2 \rrbracket+7 \llbracket 1,0,0,1 \rrbracket+5 \llbracket 1,0,0,0 \rrbracket+\llbracket 0,2,0,0 \rrbracket+\llbracket 0,1,1,0 \rrbracket+4 \llbracket 0,1,0,1 \rrbracket \\
\quad+5 \llbracket 0,1,0,0 \rrbracket+\llbracket 0,0,1,1 \rrbracket+2 \llbracket 0,0,1,0 \rrbracket+3 \llbracket 0,0,0,2 \rrbracket+4 \llbracket 0,0,0,1 \rrbracket+\llbracket 0,0,0,0 \rrbracket\end{array}$ \\
\hline
\end{tabular}

\subsubsection{The total number of states at a given mass level}

The total number of states at a given mass level $m$ can be read off from the coefficient of $q^{m}$ in the partition function $\chi^{\mathcal{N}_{10 d}=1}(q ; \boldsymbol{y})$ when the $S O(9)$ fugacities $y_{1}, \ldots, y_{4}$ are set to unity. The function $\chi^{\mathcal{N}_{10 d}=1}\left(q ;\left\{y_{i}=1\right\}\right)$ is referred to as the unrefined partition function. From (2.71), (6.3) and SUSY, ${ }^{30}$ we have

$$
\chi^{\mathcal{N}_{10 d}=1}\left(q ;\left\{y_{i}=1\right\}\right)=\left.2 \chi_{\mathrm{R}}^{\mathcal{N}_{10 d}=1}\right|_{\mathrm{GSO}}\left(q ;\left\{y_{i}=1\right\}\right)=\frac{\vartheta_{2}(1, q)^{4}}{\eta(q)^{12}}=16 \prod_{n=1}^{\infty}\left(\frac{1+q^{n}}{1-q^{n}}\right)^{8} .
$$

The coefficients in the power series of this formula reproduces the third column of Table 1 . It also agrees with (5.3.37) of [25]. Note that $\chi^{\mathcal{N}_{10 d}=1}\left(q ;\left\{y_{i}=1\right\}\right)$ is not a modular form.

\subsubsection{The number of states at each mass level and its asymptotics}

The number of states at the mass level $m$ can be determined by

$$
N_{m}=\frac{1}{2 \pi i} \oint_{\mathcal{C}} \frac{\mathrm{d} q}{q^{m+1}} \chi^{\mathcal{N}_{10 d}=1}\left(q ;\left\{y_{i}=1\right\}\right),
$$

where $\mathcal{C}$ is a contour around the origin.

Now let us compute an asymptotic formula for the number of states $N_{m}$ at mass level $m$ when $m \rightarrow \infty$. Note that a similar discussion can be found in Sections 4.3.3 and 5.3.1 of [25]. For completeness, let us go over some details here. We focus on the limit $q \rightarrow 1^{-}$and proceed in

$\overline{30}$ The agreement of GSO projected partition functions for NS and R sectors follows from Jacobi's abstruse identity:

$$
\vartheta_{3}(1, q)^{4}-\vartheta_{4}(1, q)^{4}-\vartheta_{2}(1, q)^{4}=0 .
$$


a similar way to Section 4.1. The asymptotic behavior $(4.11)$ and $(4.13)$ of $\vartheta_{2}(1, q)$ and $\eta(q)$, respectively, leads to

$$
\chi^{\mathcal{N}_{10 d}=1}\left(q ;\left\{y_{i}=1\right\}\right) \sim \frac{1}{(2 \pi)^{4}}(1-q)^{4} \exp \left(-\frac{2 \pi^{2}}{\log q}\right), \quad q \rightarrow 1^{-} .
$$

Let us now combine (6.7) with (6.8). As $m \rightarrow \infty$,

$$
N_{m} \sim \frac{1}{(2 \pi)^{4}} \frac{1}{2 \pi i} \oint_{\mathcal{C}} \frac{\mathrm{d} q}{q}(1-q)^{4} \exp \left(-\frac{2 \pi^{2}}{\log q}-m \log q\right) .
$$

The saddle point is at $q_{0}=\exp (-\pi \sqrt{2 / m})$ and the steepest descent direction is the imaginary direction in $q$. We proceed in a similar way to (4.17) by writing $q=q_{0} e^{i \theta}$ and using Laplace's method to obtain

$$
\begin{aligned}
N_{m} & \sim \frac{1}{4} m^{-2} \exp (2 \pi \sqrt{2 m}) \frac{1}{2 \pi} \int_{-\infty}^{\infty} \mathrm{d} \theta \exp \left(-\frac{m^{3 / 2}}{\pi \sqrt{2}} \theta^{2}\right) \\
& \sim \frac{1}{2^{11 / 4}} m^{-11 / 4} e^{2 \pi \sqrt{2 m}}, \quad m \rightarrow \infty .
\end{aligned}
$$

\subsubsection{The GSO projected NS and R sectors}

In this section we compute the contributions from the NS and R sectors to the partition function given in (6.3). Here we consider the refined partition function, i.e. the fugacities $y$ 's are kept explicit.

\section{The NS sector}

From (6.3) and (2.69), the partition function of the GSO projected NS sector has the structure

$$
\left.\chi_{\mathrm{NS}}^{\mathcal{N}_{10 d}=1}\right|_{\mathrm{GSO}}(q ; y)=\sum_{k \in \mathbb{Z}_{\geqslant 0}^{4}} F_{k_{1}, \ldots, k_{4}}^{\mathrm{NS}}(q) \prod_{A=1}^{4}\left[2 k_{A}\right]_{y_{A}},
$$

where the functions $F_{k_{1}, \ldots, k_{4}}^{\mathrm{NS}}(q)$ are given by

$$
\begin{aligned}
F_{k_{1}, \ldots, k_{4}}^{\mathrm{NS}}(q)= & (q ; q)_{\infty}^{-12} \sum_{\boldsymbol{n} \in \mathbb{Z}_{+}^{4}} \sum_{\boldsymbol{m} \in \mathbb{Z}_{\geqslant 0}^{4}} \\
& \times \prod_{A=1}^{4}(-1)^{n_{A}+1}\left(1-q^{n_{A}}\right) q^{\frac{1}{2} m_{A}^{2}+\left(\begin{array}{c}
n_{A} \\
2
\end{array}\right)}\left(q^{n_{A}\left|k_{A}-m_{A}\right|}-q^{n_{A}\left(k_{A}+m_{A}+1\right)}\right) \\
& \times \frac{1}{2}\left[\prod_{A=1}^{4}\left(1-q^{m_{A}+\frac{1}{2}}\right)+(-1)^{m_{1}^{2}+m_{2}^{2}+m_{3}^{2}+m_{4}^{2}} \prod_{A=1}^{4}\left(1+q^{m_{A}+\frac{1}{2}}\right)\right] .
\end{aligned}
$$

\section{The $R$ sector}

From (6.3) and (2.70), the partition function of the GSO projected R sector is

$$
\left.\chi_{\mathrm{R}}^{\mathcal{N}_{10 d}=1}\right|_{\mathrm{GSO}}(q ; y, s)=\sum_{\boldsymbol{k} \in \mathbb{Z}_{\geqslant 0}^{4}} F_{k_{1}, \ldots, k_{4}}^{\mathrm{R}}(q) \prod_{A=1}^{4}\left[2 k_{A}+1\right]_{y_{A}},
$$


where the function $F_{k_{1}, \ldots, k_{4}}^{\mathrm{R}}(q)$ is given by

$$
\begin{aligned}
& F_{k_{1}, \ldots, k_{4}}^{\mathrm{R}}(q) \\
& =\frac{1}{2} q^{-\frac{1}{2}}(q ; q)_{\infty}^{-12} \sum_{\boldsymbol{m} \in \mathbb{Z}_{\geqslant 0}^{4}} \sum_{\boldsymbol{n} \in \mathbb{Z}_{+}^{4}} \prod_{A=1}^{4}(-1)^{n_{A}+1}\left(1-q^{m_{A}+1}\right)\left(1-q^{n_{A}}\right) q^{\frac{1}{2}\left(m_{A}+\frac{1}{2}\right)^{2}+\left(\begin{array}{c}
n_{A} \\
2
\end{array}\right)} \\
& \quad \times \prod_{A=1}^{4}\left(q^{n_{A}\left|k_{A}-m_{A}\right|}-q^{n_{A}\left(k_{A}+m_{A}+2\right)}\right)
\end{aligned}
$$

6.1.4. Multiplicities of representations in the $\mathcal{N}_{10 d}=1$ partition function

Combining the contributions from the NS and R sectors, we have

$$
\begin{aligned}
\chi^{\mathcal{N}_{10 d}=1}(q ; \boldsymbol{y}) & =\left.\chi_{\mathrm{NS}}^{\mathcal{N}_{10 d}=1}\right|_{\mathrm{GSO}}(q ; \boldsymbol{y})+\left.\chi_{\mathrm{R}}^{\mathcal{N}_{10 d}=1}\right|_{\mathrm{GSO}}(q ; \boldsymbol{y}) \\
& =\sum_{\boldsymbol{k} \in \mathbb{Z}_{\geqslant 0}^{4}}\left(F_{\boldsymbol{k}}^{\mathrm{NS}}(q) \prod_{A=1}^{4}\left[2 k_{A}\right]_{y_{A}}+F_{\boldsymbol{k}}^{\mathrm{R}} \prod_{A=1}^{4}\left[2 k_{A}+1\right]_{y_{A}}\right) .
\end{aligned}
$$

Supersymmetry implies that this partition function can be rewritten as

$$
\chi^{\mathcal{N}_{10 d}=1}(q ; \boldsymbol{y})=\sum_{\boldsymbol{n} \in \mathbb{Z}_{\geqslant 0}^{4}} \llbracket n_{1}, n_{2}, n_{3}, n_{4} \rrbracket G_{n_{1}, n_{2}, n_{3}, n_{4}}(q),
$$

and the aim is to compute explicitly a multiplicity generating function $G_{n_{1}, n_{2}, n_{3}, n_{4}}(q)$.

The multiplicity of $\llbracket n_{1}, n_{2}, n_{3}, n_{4} \rrbracket$ appearing in $\chi^{\mathcal{N}_{10 d}=1}(q ; \boldsymbol{y})$ can be determined as follows:

$$
\begin{aligned}
G_{n_{1}, n_{2}, n_{3}, n_{4}}(q) & =\int \mathrm{d} \mu_{S O(9)}(\boldsymbol{y})\left[n_{1}, n_{2}, n_{3}, n_{4}\right] y \frac{\chi^{\mathcal{N}_{10 d}=1}(q ; \boldsymbol{y})}{Z\left(\mathcal{N}_{10 d}=1\right)(\boldsymbol{y})} \\
& =G_{n_{1}, n_{2}, n_{3}, n_{4}}^{\mathrm{NS}}(q)+G_{n_{1}, n_{2}, n_{3}, n_{4}}^{\mathrm{R}}(q),
\end{aligned}
$$

where

$$
\begin{aligned}
& G_{n_{1}, n_{2}, n_{3}, n_{4}}^{\mathrm{NS}}(q) \\
& \quad=\int \mathrm{d} \mu_{S O(9)}(\boldsymbol{y})\left[n_{1}, n_{2}, n_{3}, n_{4}\right]_{\boldsymbol{y}} \sum_{\boldsymbol{k} \in \mathbb{Z}_{\geqslant 0}^{4}} \frac{\prod_{A=1}^{4}\left[2 k_{A}\right]_{y_{A}}}{Z\left(\mathcal{N}_{10 d}=1\right)(\boldsymbol{y})} F_{k_{1}, \ldots, k_{4}}^{\mathrm{NS}}(q), \\
& G_{n_{1}, n_{2}, n_{3}, n_{4}}^{\mathrm{R}}(q) \\
& \quad=\int \mathrm{d} \mu_{S O(9)}(\boldsymbol{y})\left[n_{1}, n_{2}, n_{3}, n_{4}\right]_{\boldsymbol{y}} \sum_{\boldsymbol{k} \in \mathbb{Z}_{\geqslant 0}^{4}} \frac{\prod_{A=1}^{4}\left[2 k_{A}+1\right]_{y_{A}}}{Z\left(\mathcal{N}_{10 d}=1\right)(\boldsymbol{y})} F_{k_{1}, \ldots, k_{4}}^{\mathrm{R}}(q) .
\end{aligned}
$$

The inverse of the character of the fundamental multiplet in (6.1) can be written as a geometric 
series $^{31}$ similar to (4.42) and (5.29)

$$
\begin{aligned}
& {\left[Z\left(\mathcal{N}_{10 d}=1\right)(\boldsymbol{y}, r)\right]^{-1}} \\
& \quad=\frac{y_{4}^{4}}{\left(1+\frac{y_{4}}{y_{1} y_{2} y_{3}}\right)\left(1+\frac{y_{1} y_{4}}{y_{2} y_{3}}\right)\left(1+\frac{y_{2} y_{4}}{y_{1} y_{3}}\right)\left(1+\frac{y_{1} y_{2} y_{4}}{y_{3}}\right)\left(1+\frac{y_{3} y_{4}}{y_{1} y_{2}}\right)\left(1+\frac{y_{1} y_{3} y_{4}}{y_{2}}\right)\left(1+\frac{y_{2} y_{3} y_{4}}{y_{1}}\right)\left(1+y_{1} y_{2} y_{3} y_{4}\right)} \\
& \quad=\sum_{\boldsymbol{m} \in \mathbb{Z}_{\geqslant 0}^{8}}(-1)^{\sum_{j=1}^{8} m_{j}} y_{1}^{\sum_{j=1}^{8}(-1)^{j} m_{j}} y_{2}^{\sum_{j=1}^{8}(-1)^{\lfloor(j+1) / 2\rfloor} m_{j}} y_{3}^{\sum_{j=1}^{8}(-1)^{\lfloor(j+3) / 4\rfloor} m_{j}} y_{4}^{4+\sum_{j=1}^{8} m_{j}} .
\end{aligned}
$$

\subsubsection{Some useful identities}

In this section, we derive some useful identities that will be put into use later. Once we plug the series expansion (6.21) of the inverse $Z\left(\mathcal{N}_{10 d}=1\right)$ into the integrand of (6.17), the elementary contributions to multiplicity generating functions $G_{n_{1}, n_{2}, n_{3}, n_{4}}$ are integrals of type

$$
\mathcal{J}_{0}(w ; \boldsymbol{p}):=\int \mathrm{d} \mu_{S O(3)}(r) r^{w} \prod_{A=1}^{4}\left[p_{A}\right]_{r}
$$

as well as

$$
\mathcal{J}(\boldsymbol{w} ; \boldsymbol{k} ; \boldsymbol{n}):=\int \mathrm{d} \mu_{S O(9)}(\boldsymbol{y})\left[n_{1}, n_{2}, n_{3}, n_{4}\right]_{\boldsymbol{y}} \prod_{A=1}^{4} y_{A}^{w_{A}}\left[k_{A}\right]_{y_{A}} .
$$

There are four cases to be considered, namely spin/non-spin representations of $S O(9)$ and for each of these cases $k_{1}, \ldots, k_{4}$ can be all even or all odd. In what follows, we assume that $\boldsymbol{k}, \boldsymbol{n} \in$ $\mathbb{Z}_{\geqslant 0}^{4}$ and $\boldsymbol{w} \in \mathbb{Z}^{4}$. For non-spin representations,

$$
\begin{aligned}
\mathcal{J} & \left(\boldsymbol{w} ; 2 k_{1}, \ldots, 2 k_{4} ; n_{1}, \ldots, 2 n_{4}\right) \\
& =\sum_{\boldsymbol{k}^{\prime} \in \mathbb{Z}_{\geqslant 0}^{4}} \Delta\left(\lambda_{n s} ; 2 k_{1}^{\prime}, \ldots, 2 k_{4}^{\prime}\right) \prod_{A=1}^{4} \mathcal{J}_{0}\left(w_{A} ; 2 k_{A}, 2 k_{A}^{\prime}\right), \\
\mathcal{J} & \left(\boldsymbol{w} ; 2 k_{1}+1, \ldots, 2 k_{4}+1 ; n_{1}, \ldots, 2 n_{4}\right) \\
& =\sum_{\boldsymbol{k}^{\prime} \in \mathbb{Z}_{\geqslant 0}^{4}} \Delta\left(\lambda_{n s} ; 2 k_{1}^{\prime}, \ldots, 2 k_{4}^{\prime}\right) \prod_{A=1}^{4} \mathcal{J}_{0}\left(w_{A} ; 2 k_{A}+1,2 k_{A}^{\prime}\right),
\end{aligned}
$$

where $\lambda_{n s}=\left(n_{1}+n_{2}+n_{3}+n_{4}, n_{2}+n_{3}+n_{4}, n_{3}+n_{4}, n_{4}\right)$. For spin representations,

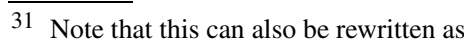

$$
\begin{aligned}
{\left[Z\left(\mathcal{N}_{10 d}=1\right)(\boldsymbol{y})\right]^{-1} } & =\lim _{s \rightarrow-1}(\operatorname{PE}[s[0,0,0,1] \boldsymbol{y}])^{1 / 2} \\
& =\left[\sum_{m=0}^{\infty}(-1)^{m} \operatorname{Sym}^{m}[0,0,0,1] \boldsymbol{y}\right]^{1 / 2} .
\end{aligned}
$$




$$
\begin{aligned}
\mathcal{J} & \left(\boldsymbol{w} ; 2 k_{1}, \ldots, 2 k_{4} ; n_{1}, \ldots, 2 n_{4}+1\right) \\
& =\sum_{\boldsymbol{k}^{\prime} \in \mathbb{Z}^{4}} \Delta\left(\lambda_{s} ; 2 k_{1}^{\prime}+1, \ldots, 2 k_{4}^{\prime}+1\right) \prod_{A=1}^{4} \mathcal{J}_{0}\left(w_{A} ; 2 k_{A}, 2 k_{A}^{\prime}+1\right), \\
\mathcal{J} & \left(\boldsymbol{w} ; 2 k_{1}+1, \ldots, 2 k_{4}+1 ; n_{1}, \ldots, 2 n_{4}+1\right) \\
& =\sum_{\boldsymbol{k}^{\prime} \in \mathbb{Z}_{\geqslant 0}^{4}} \Delta\left(\lambda_{s} ; 2 k_{1}^{\prime}+1, \ldots, 2 k_{4}^{\prime}+1\right) \prod_{A=1}^{4} \mathcal{J}_{0}\left(w_{A} ; 2 k_{A}+1,2 k_{A}^{\prime}+1\right),
\end{aligned}
$$

where $\lambda_{s}=\left(n_{1}+n_{2}+n_{3}+n_{4}+\frac{1}{2}, n_{2}+n_{3}+n_{4}+\frac{1}{2}, n_{3}+n_{4}+\frac{1}{2}, n_{4}+\frac{1}{2}\right)$. Recall from (2.57) and (2.62) that

$$
\begin{aligned}
& \Delta\left(\lambda ; 2 k_{1}, \ldots, 2 k_{4}\right)=\frac{1}{4 !} \sum_{\sigma \in S_{4}} \operatorname{det}\left(\theta_{\left|\lambda_{A}-A+B\right|}^{8+\lambda_{A}-A-B}\left(k_{\sigma(A)}\right)\right)_{A, B=1}^{4}, \\
& \Delta\left(\lambda ; 2 k_{1}+1, \ldots, 2 k_{4}+1\right)=\frac{1}{4 !} \sum_{\sigma \in S_{4}} \operatorname{det}\left(\theta_{\left|\lambda_{A}-A+B\right|}^{8+\lambda_{A}-A-B}\left(k_{\sigma(A)}+\frac{1}{2}\right)\right)_{A, B=1}^{4} .
\end{aligned}
$$

\subsubsection{Multiplicity generating function}

The NS and R sector contributions to the multiplicity generating function for the representation $\llbracket n_{1}, n_{2}, n_{3}, n_{4} \rrbracket$ can be rewritten as

$$
\begin{aligned}
& G_{n_{1}, \ldots, n_{4}}^{\mathrm{NS}}(q)=\sum_{\boldsymbol{m} \in \mathbb{Z}_{\geqslant 0}^{8}}(-1)^{\sum_{j=1}^{8} m_{j}} \sum_{\boldsymbol{k} \in \mathbb{Z}_{\geqslant 0}^{4}} \mathcal{J}\left(\boldsymbol{W}(\boldsymbol{m}) ; 2 k_{1}, \ldots, 2 k_{4} ; \boldsymbol{n}\right) F_{k_{1}, \ldots, k_{4}}^{\mathrm{NS}}(q), \\
& G_{n_{1}, \ldots, n_{4}}^{\mathrm{R}}(q)=\sum_{\boldsymbol{m} \in \mathbb{Z}_{\geqslant 0}^{8}}(-1)^{\sum_{j=1}^{8} m_{j}} \sum_{\boldsymbol{k} \in \mathbb{Z}_{\geqslant 0}^{4}} \mathcal{J}\left(\boldsymbol{W}(\boldsymbol{m}) ; 2 k_{1}+1, \ldots, 2 k_{4}+1 ; \boldsymbol{n}\right) F_{k_{1}, \ldots, k_{4}}^{\mathrm{R}}(q),
\end{aligned}
$$

where

$$
\boldsymbol{W}(\boldsymbol{m})=\left(\sum_{j=1}^{8}(-1)^{j} m_{j}, \sum_{j=1}^{8}(-1)^{\lfloor(j+1) / 2\rfloor} m_{j}, \sum_{j=1}^{8}(-1)^{\lfloor(j+3) / 4\rfloor} m_{j}, 4+\sum_{j=1}^{8} m_{j}\right) .
$$

As stated in (6.3), the multiplicity of the representation $\llbracket n_{1}, n_{2}, n_{3}, n_{4} \rrbracket$ in the $\mathcal{N}_{10 d}=1$ partition function is given by

$$
\begin{aligned}
& G_{n_{1}, n_{2}, n_{3}, n_{4}}(q) \\
& =\sum_{\boldsymbol{m} \in \mathbb{Z}_{\geqslant 0}^{8}}(-1)^{\sum_{j=1}^{8} m_{j}} \sum_{\boldsymbol{k} \in \mathbb{Z}_{\geqslant 0}^{4}}\left[\mathcal{J}\left(\boldsymbol{W}(\boldsymbol{m}) ; 2 k_{1}, \ldots, 2 k_{4} ; \boldsymbol{n}\right) F_{k_{1}, \ldots, k_{4}}^{\mathrm{NS}}(q)\right. \\
& \left.\quad+\mathcal{J}\left(\boldsymbol{W}(\boldsymbol{m}) ; 2 k_{1}+1, \ldots, 2 k_{4}+1 ; \boldsymbol{n}\right) F_{k_{1}, \ldots, k_{4}}^{\mathrm{R}}(q)\right] .
\end{aligned}
$$

\subsection{Empirical approach to $\mathcal{N}_{10 d}=1$ asymptotic patterns}

In this subsection, we proceed like in Sections 4.5 and 5.4 to obtain large spin asymptotics of multiplicity generating functions $G_{n, x, y, z}(q)$ for $\mathcal{N}_{10 d}=1$ supermultiplet $\llbracket n, x, y, z \rrbracket$. The 
supermultiplet content of the first 25 mass levels is used to determine the $q$ expansion of the leading coefficients $\tau_{\ell}^{x, y, z}(q)$ defined by:

$$
\begin{aligned}
G_{n, x, y, z}(q) & =q^{n} \tau_{1}^{x, y, z}(q)-q^{2 n} \tau_{2}^{x, y, z}(q)+q^{3 n} \tau_{3}^{x, y, z}(q)-\cdots \\
& =\sum_{\ell=1}^{\infty}(-1)^{\ell-1} q^{\ell n} \tau_{\ell}^{x, y, z}(q) .
\end{aligned}
$$

Again, the $\tau_{\ell}^{x, y, z}(q)$ are found to be power series in $q$ with non-negative coefficients.

Having $d>4$ spacetime dimensions makes the analytic methods of Section 4.4 inefficient, i.e. we did not find a manageable asymptotic formula for (6.33). Hence, we compute the $\tau_{\ell}^{x, y, z}(q)$ at $\ell \leqslant 5$ on the basis of an $\mathcal{O}\left(q^{25}\right)$ expansion of the partition function (6.3). The multiplicities of $\llbracket n, 0,0,0 \rrbracket$ multiplets are shown in the following Table 9, and analogous data tables for $\llbracket n, x, y, z \rrbracket$ at non-zero values of $x, y, z$ can be found in Appendix B.3. The numbers marked in red match with the leading trajectory contribution $q^{n} \tau_{1}^{x, y, z}(q)$ whereas blue numbers correspond to $q^{n} \tau_{1}^{x, y, z}(q)-q^{2 n} \tau_{2}^{x, y, z}(q)$ including one subleading trajectory.

\subsubsection{Levels of first appearance}

The mass level where some $\llbracket 0, x, y, z \rrbracket$ multiplet firstly occurs can be studied by inspecting the leading power of the multiplicity generating function $G_{0, x, y, z}(q)$ and therefore $\tau_{\ell}^{x, y, z}(q)$. The following Table 10 gives an overview of this mass level threshold for various values of $x, y, z$.

For all supermultiplets $\llbracket 0, x, y, z \rrbracket$ considered in Table 10, the level of first appearance is delayed by three whenever the second Dynkin label is incremented as $x \mapsto x+1$. This suggests to look for a similar linear effect of $y \mapsto y+1$ and $z \mapsto z+1$. Up to the two exceptions $\llbracket 0,0,0,0 \rrbracket$ and $\llbracket 0,0,0,1 \rrbracket$, the data in the tables shows that the value $y$ of the third Dynkin label increases the level of first appearance by $6 y$.

The influence of the last Dynkin label $z$ is much more difficult to probe without any explicit multiplicities beyond level 25 at hand. If an asymptotically linear relation between $z$ and the level of first appearance of $\llbracket 0, x, y, z \rrbracket$ exists, then it certainly admits even more exceptions than in the $y \mapsto y+1$ case. The onset of $\llbracket n, 0,0,4 \rrbracket, \llbracket n, 0,0,5 \rrbracket$ and $\llbracket n, 0,0,6 \rrbracket$ multiplets at levels 14,19 and 24 , respectively, suggests that an increment $z \mapsto z+1$ delays the $\llbracket 0, x, y, z \rrbracket$ multiplet by five levels - at least in the regime of sufficiently large values of $x, y, z$.

On the basis of this reasoning, we conjecture that sufficiently high mass levels of first occurrence for general supermultiplets $\llbracket n, x, y, z \rrbracket$ are determined by the following overall prefactor in their multiplicity generating function:

$$
G_{n, x, y, z}(q) \sim q^{n+3 x+6 y+5 z-6} \times \mathcal{O}(1), \quad x, y, z \text { large }
$$

Note that also the six-dimensional $\mathcal{N}_{6 d}=(1,0)$ spectrum exhibits an asymptotic linear relation between the second $S O(5)$ label $k$ and the level of first appearance: Table 6 shows that sufficiently high levels of first appearance for $\llbracket n, k ; p \rrbracket$ are delayed by three under $k \mapsto k+2$.

\subsubsection{Explicit formulae for the $\tau_{\ell}^{x, y, z}(q)$}

We shall now give the explicit results for a large class of $\tau_{\ell}^{x, y, z}(q)$, obtained through the entries of Table 9 and its generalizations to $(x, y, z) \neq(0,0,0)$ gathered in Appendix B.3. This reflects large spin information on the multiplicity generating functions $G_{n, x, y, z}(q)$ via (6.34). 
Table 9

$\mathcal{N}_{10 d}=1$ multiplets with $S O(9)$ quantum numbers $[n, 0,0,0]$.

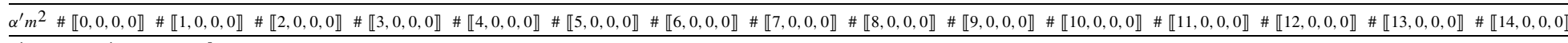

$\begin{array}{rrr}1 & 0 & \\ 0 & 1 & 0 \\ 0 & 0 & 1 \\ 0 & 1 & 0 \\ 1 & 0 & 1 \\ 0 & 2 & 1 \\ 2 & 2 & 3 \\ 1 & 5 & 3 \\ 3 & 5 & 9 \\ 3 & 12 & 10 \\ 8 & 15 & 23 \\ 8 & 30 & 31 \\ 19 & 41 & 61 \\ 22 & 77 & 89 \\ 41 & 109 & 164 \\ 57 & 190 & 245 \\ 100 & 282 & 426 \\ 138 & 471 & 656 \\ 235 & 710 & 1097 \\ 336 & 1153 & 1999 \\ 544 & 1750 & 2778 \\ 799 & 2785 & 4309 \\ 1261 & 4237 & 6907 \\ 1860 & 6634 & 10700 \\ 2895 & 10082 & 16893 \\ & & \end{array}$

$\begin{array}{r}0 \\ 1 \\ 0 \\ 1 \\ 1 \\ 4 \\ 4 \\ 11 \\ 14 \\ 31 \\ 45 \\ 87 \\ 132 \\ 244 \\ 378 \\ 657 \\ 1040 \\ 1751 \\ 2769 \\ 4561 \\ 7201 \\ 11637 \\ 18301 \\ \hline\end{array}$

\begin{tabular}{|c|c|c|c|c|}
\hline 0 & & & & \\
\hline 1 & 0 & & & \\
\hline 0 & 1 & 0 & & \\
\hline 1 & $\begin{array}{l}1 \\
0\end{array}$ & 1 & 0 & \\
\hline 1 & 1 & $\begin{array}{l}1 \\
0\end{array}$ & 1 & 0 \\
\hline $\begin{array}{l}1 \\
4\end{array}$ & 1 & 1 & $\begin{array}{l}1 \\
0\end{array}$ & 1 \\
\hline 5 & 4 & 1 & 1 & 0 \\
\hline 12 & 5 & 4 & 1 & 1 \\
\hline $\begin{array}{l}16 \\
16\end{array}$ & 13 & 5 & 4 & 1 \\
\hline $\begin{array}{l}10 \\
36\end{array}$ & 17 & 13 & 5 & 4 \\
\hline 53 & $\begin{array}{l}11 \\
38\end{array}$ & 18 & 13 & 5 \\
\hline 104 & 58 & 39 & 18 & 13 \\
\hline 162 & 113 & 60 & 40 & 18 \\
\hline 299 & 179 & 118 & 61 & 40 \\
\hline 473 & 332 & 188 & 120 & 62 \\
\hline 830 & 532 & 350 & 193 & 121 \\
\hline 1333 & 938 & 565 & 359 & 195 \\
\hline 2263 & 1523 & 1000 & 583 & 364 \\
\hline 3630 & 2600 & 1635 & 1034 & 592 \\
\hline $\begin{array}{l}6025 \\
6025\end{array}$ & 4212 & 2803 & $\begin{array}{l}1054 \\
1697\end{array}$ & $\begin{array}{r}3052 \\
\end{array}$ \\
\hline 9629 & 7034 & 4567 & 2918 & 1731 \\
\hline 15694 & 11337 & 7662 & 4774 & 2981 \\
\hline
\end{tabular}

$\begin{array}{rr}0 & \\ 1 & 0 \\ 0 & 1 \\ 1 & 0 \\ 1 & 1 \\ 4 & 1 \\ 5 & 4 \\ 13 & 5 \\ 18 & 13 \\ 40 & 18 \\ 62 & 40 \\ 122 & 62 \\ 196 & 122 \\ 366 & 197 \\ 597 & 367 \\ 1061 & 599 \\ 1749 & 1066\end{array}$


Table 10

First mass level where fermionic supermultiplets $\llbracket 0, x, y, z \rrbracket$ of $\mathcal{N}_{10 d}=1$ firstly occur. Empty spaces indicate that the representations in question do not occur at levels $\leqslant 25$.

\begin{tabular}{llllllll}
\hline$\downarrow y, \vec{z}$ & 0 & 1 & 2 & 3 & 4 & 5 & 6 \\
\hline 0 & $1+3 x$ & $3+3 x$ & $6+3 x$ & $10+3 x$ & $14+3 x$ & $19+3 x$ & $24+3 x$ \\
1 & $5+3 x$ & $8+3 x$ & $12+3 x$ & $16+3 x$ & $20+3 x$ & $25+3 x$ & \\
2 & $11+3 x$ & $14+3 x$ & $18+3 x$ & $22+3 x$ & & & \\
3 & $17+3 x$ & $20+3 x$ & $24+3 x$ & & & & \\
4 & $23+3 x$ & & & & & & \\
\hline
\end{tabular}

- $S O(9)$ Dynkin labels $[n \rightarrow \infty, 0,0,0]$

$$
\begin{aligned}
\tau_{1}^{0,0,0}(q)= & q^{1}\left(1+0 q+1 q^{2}+1 q^{3}+4 q^{4}+5 q^{5}+13 q^{6}+18 q^{7}+40 q^{8}+62 q^{9}\right. \\
& +122 q^{10}+197 q^{11}+368 q^{12}+601 q^{13}+1070 q^{14}+1767 q^{15} \\
& \left.+3051 q^{16}+5022 q^{17}+8489 q^{18}+13897 q^{19}+\cdots\right), \\
\tau_{2}^{0,0,0}(q)= & q^{1}\left(1+2 q+4 q^{2}+9 q^{3}+18 q^{4}+36 q^{5}+70 q^{6}+133 q^{7}+249 q^{8}\right. \\
& \left.+460 q^{9}+836 q^{10}+1503 q^{11}+2672 q^{12}+4699 q^{13}+\cdots\right), \\
\tau_{3}^{0,0,0}(q)= & q^{1}\left(1+1 q+5 q^{2}+9 q^{3}+26 q^{4}+48 q^{5}+112 q^{6}+211 q^{7}\right. \\
& \left.+439 q^{8}+818 q^{9}+\cdots\right), \\
\tau_{4}^{0,0,0}(q)= & q^{1}\left(1+3 q+8 q^{2}+20 q^{3}+48 q^{4}+106 q^{5}+\cdots\right), \\
\tau_{5}^{0,0,0}(q)= & q^{1}\left(1+1 q+6 q^{2}+\cdots\right) .
\end{aligned}
$$

- $S O(9)$ Dynkin labels $[n \rightarrow \infty, 1,0,0]$

$$
\begin{aligned}
\tau_{1}^{1,0,0}(q)= & q^{4}\left(1+2 q+3 q^{2}+7 q^{3}+14 q^{4}+28 q^{5}+53 q^{6}+103 q^{7}+189 q^{8}\right. \\
& +352 q^{9}+634 q^{10}+1146 q^{11}+2026 q^{12}+3578 q^{13}+6209 q^{14} \\
& \left.+10752 q^{15}+18378 q^{16}+31279 q^{17}+\cdots\right), \\
\tau_{2}^{1,0,0}(q)= & q^{5}\left(1+2 q+5 q^{2}+11 q^{3}+26 q^{4}+54 q^{5}+114 q^{6}+227 q^{7}+449 q^{8}\right. \\
& +863 q^{9}+1639 q^{10}+3050 q^{11} \\
& \left.+5618 q^{12}+10187 q^{13}+\cdots\right), \\
\tau_{3}^{1,0,0}(q)= & q^{8}\left(2+5 q+15 q^{2}+35 q^{3}+86 q^{4}+185 q^{5}+403 q^{6}+825 q^{7}+\cdots\right), \\
\tau_{4}^{1,0,0}(q)= & q^{10}\left(1+3 q+11 q^{2}+30 q^{3}+\cdots\right) .
\end{aligned}
$$

- $S O(9)$ Dynkin labels $[n \rightarrow \infty, 0,1,0]$

$$
\begin{aligned}
\tau_{1}^{0,1,0}(q)= & q^{5}\left(1+1 q+5 q^{2}+8 q^{3}+22 q^{4}+40 q^{5}+90 q^{6}+165 q^{7}+338 q^{8}\right. \\
& +619 q^{9}+1190 q^{10}+2149 q^{11}+3969 q^{12}+7048 q^{13}+12630 q^{14} \\
& \left.+22060 q^{15}+38603 q^{16}+\cdots\right), \\
\tau_{2}^{0,1,0}(q)= & q^{6}\left(1+2 q+7 q^{2}+17 q^{3}+41 q^{4}+91 q^{5}+199 q^{6}+412 q^{7}\right. \\
& \left.+841 q^{8}+1665 q^{9}+3241 q^{10}+6178 q^{11}+11611 q^{12}+\cdots\right), \\
\tau_{3}^{0,1,0}(q)= & q^{8}\left(1+2 q+11 q^{2}+25 q^{3}+71 q^{4}+160 q^{5}+381 q^{6}+809 q^{7}+\cdots\right),
\end{aligned}
$$




$$
\tau_{4}^{0,1,0}(q)=q^{11}\left(2+7 q+23 q^{2}+\cdots\right) .
$$

- $S O(9)$ Dynkin labels $[n \rightarrow \infty, 0,0,2]$

$$
\begin{aligned}
\tau_{1}^{0,0,2}(q)= & q^{6}\left(1+2 q+7 q^{2}+13 q^{3}+33 q^{4}+66 q^{5}+143 q^{6}+277 q^{7}\right. \\
& +559 q^{8}+1053 q^{9}+2019 q^{10}+3715 q^{11}+6859 q^{12}+12338 q^{13} \\
& \left.+22156 q^{14}+39043 q^{15}+\cdots\right), \\
\tau_{2}^{0,0,2}(q)= & q^{7}\left(1+4 q^{1}+11 q^{2}+28 q^{3}+68 q^{4}+155 q^{5}+339 q^{6}+716 q^{7}+1469 q^{8}\right. \\
& \left.+2938 q^{9}+5755 q^{10}+11054 q^{11}+\cdots\right), \\
\tau_{3}^{0,0,2}(q)= & q^{9}\left(2+5 q+19 q^{2}+48 q^{3}+130 q^{4}+301 q^{5}+703 q^{6}+1518 q^{7}+\cdots\right), \\
\tau_{4}^{0,0,2}(q)= & q^{11}\left(1+4 q+16 q^{2}+49 q^{3}+\cdots\right) .
\end{aligned}
$$

- $S O(9)$ Dynkin labels $[n \rightarrow \infty, 0,0,1]$

$$
\begin{aligned}
\tau_{1}^{0,0,1}(q)= & q^{3}\left(1+1 q+3 q^{2}+6 q^{3}+12 q^{4}+24 q^{5}+48 q^{6}+90 q^{7}+171 q^{8}\right. \\
& +317 q^{9}+579 q^{10}+1045 q^{11}+1870 q^{12}+3299 q^{13}+5777 q^{14} \\
& \left.+10017 q^{15}+17222 q^{16}+29370 q^{17}+\cdots\right), \\
\tau_{2}^{0,0,1}(q)= & q^{4}\left(1+2 q^{1}+5 q^{2}+13 q^{3}+29 q^{4}+62 q^{5}+130 q^{6}+263 q^{7}\right. \\
& +520 q^{8}+1008 q^{9}+1916 q^{10} \\
& \left.+3583 q^{11}+6609 q^{12}+\cdots\right) \\
\tau_{3}^{0,0,1}(q)= & q^{6}\left(1+3 q^{1}+10 q^{2}+26 q^{3}+63 q^{4}+143 q^{5}+315 q^{6}+664 q^{7}+\cdots\right), \\
\tau_{4}^{0,0,1}(q)= & q^{8}\left(1+4 q+12 q^{2}+35 q^{3}+\cdots\right) \\
\tau_{5}^{0,0,1}(q)= & q^{10}(1+\cdots) .
\end{aligned}
$$

Further $\tau_{\ell}^{x, y, z}(q)$ listed in AUXILIARY FILE 2 support the trend that the $\tau_{\ell}^{x, y, z}(q)$ expansion (6.34) converges more quickly at higher value of $x, y, z$.

\subsection{Eight-dimensional $\mathcal{N}_{8 d}=1$ spectra}

Starting from this subsection, we consider even-dimensional Type I superstring compactifications on $T^{2}$ tori preserving all the sixteen supercharges. The highest-dimensional example is $\mathcal{N}_{8 d}=1$ SUSY in eight spacetime dimensions. As explained in $[42,43]$, dimensional reduction of the open superstring from $d=10$ to $d=8$ paves the way towards powerful on-shell SUSY techniques to manifest hidden simplicity of scattering amplitudes among massive string modes (further examples following in [44]): One technical advantage of the eight-dimensional setting is the possibility to covariantly single out a Clifford vacuum which is annihilated by half of the supercharges, say the right-handed $S O(8)$ spinor of SUSY generators $[42,43]$. This is a particular motivation to focus on the covariant particle content of the maximally supersymmetric open superstring in $d=8$.

Let $r$ denote the fugacity with respect to the R symmetry $S O(2)_{R} \cong U(1)_{R}$ and $y_{i}$ the fugacities of the massive little group $S O(7)$, then the fundamental $\mathcal{N}_{8 d}=1$ super-Poincaré multiplet is described by the supercharacter 


$$
\begin{aligned}
Z\left(\mathcal{N}_{8 d}=1\right):= & \left(r^{4}+r^{-4}\right)[0,0,0]+\left(r^{3}+r^{-3}\right)[0,0,1] \\
& +\left(r^{2}+r^{-2}\right)([0,1,0]+[1,0,0]) \\
& +\left(r+r^{-1}\right)([1,0,1]+[0,0,1]) \\
& +[2,0,0]+[0,0,2]+[1,0,0]+[0,0,0]
\end{aligned}
$$

which is obtained by branching the $S O(9)$ representations contributing to the $\mathcal{N}_{10 d}=1$ analogue (6.1) to $S O(7) \times U(1)_{R}$. The minimal multiplet (6.41) can be generated from a scalar Clifford vacuum of $U(1)_{R}$ charge +4 , and the generic $\mathcal{N}_{8 d}=1$ multiplet follows from a Clifford vacuum with non-trivial $S O(7) \times U(1)_{R}$ quantum numbers. ${ }^{32}$ This gives rise to the supercharacter

$$
\llbracket a_{1}, a_{2}, a_{3} ; Q \rrbracket:=Z\left(\mathcal{N}_{8 d}=1\right) \cdot r^{Q}\left[a_{1}, a_{2}, a_{3}\right] .
$$

The eight-dimensional partition function is obtained from its ten-dimensional ancestor (6.3) by singling out an internal factor $\chi_{\mathrm{NS}, \mathrm{R}}^{S O(3)}$ within $\chi_{\mathrm{NS}, \mathrm{R}}^{S O(9)}(y)=\prod_{k=1}^{4} \chi_{\mathrm{NS}, \mathrm{R}}^{S O(3)}\left(y_{k}\right)$ and reinterpreting its argument as an $R$-symmetry fugacity:

$$
\begin{aligned}
& \chi^{\mathcal{N}_{8 d}=1}(q ; \boldsymbol{y}, r)=\left.\chi_{\mathrm{NS}}^{\mathcal{N}_{8 d}=1}\right|_{\mathrm{GSO}}(q ; \boldsymbol{y}, r)+\left.\chi_{\mathrm{R}}^{\mathcal{N}_{8 d}=1}\right|_{\mathrm{GSO}}(q ; \boldsymbol{y}, r), \\
& \left.\chi_{\mathrm{NS}}^{\mathcal{N}_{8 d}=1}\right|_{\mathrm{GSO}}(q ; \boldsymbol{y}, r)=\frac{1}{2} q^{-\frac{1}{2}}\left[\chi_{\mathrm{NS}}^{S O(7)}(q ; \boldsymbol{y}) \chi_{\mathrm{NS}}^{S O(3)}(q ; r)\right. \\
& \left.-\chi_{\mathrm{NS}}^{S O(7)}\left(e^{2 \pi i} q ; \boldsymbol{y}\right) \chi_{\mathrm{NS}}^{S O(3)}\left(e^{2 \pi i} q ; r\right)\right] \text {, } \\
& \left.\chi_{\mathrm{R}}^{\mathcal{N}_{8 d}=1}\right|_{\mathrm{GSO}}(q ; \boldsymbol{y}, r)=\frac{1}{2} \chi_{\mathrm{R}}^{S O(7)}(q ; \boldsymbol{y}) \chi_{\mathrm{R}}^{S O(3)}(q ; r) .
\end{aligned}
$$

Let us display the first four coefficients of the power series expansion in $q^{33}$ :

$$
\begin{aligned}
\chi^{\mathcal{N}_{8 d}}=1 & (q ; \boldsymbol{y}, r) \\
= & \underbrace{\left(\sum_{j=1}^{3}\left(y_{j}^{2}+y_{j}^{-2}\right)+r^{2}+r^{-2}+\frac{1}{2} \prod_{j=1}^{3}\left(y_{j}+y_{j}^{-1}\right)\left(r+r^{-1}\right)\right) q^{0}}_{16 \text { massless states }} \\
& +\underbrace{\llbracket 0,0,0 ; 0 \rrbracket q}_{256 \text { states at level } 1}+\underbrace{(\llbracket 0,0,0 ; \pm 2 \rrbracket+\llbracket 1,0,0 ; 0 \rrbracket) q^{2}}_{2304 \text { states at level } 2} \\
& +(\llbracket 0,0,0 ; \pm 4 \rrbracket+\llbracket 1,0,0 ; \pm 2 \rrbracket+\llbracket 0,0,1 ; \pm 1 \rrbracket \\
& +\llbracket 2,0,0 ; 0 \rrbracket+\llbracket 0,0,0 ; 0 \rrbracket) q^{3}+\mathcal{O}\left(q^{4}\right) .
\end{aligned}
$$

The pairing of opposite $U(1)_{R}$ charges $\pm Q$ motivates the following shorthand:

$$
\llbracket a_{1}, a_{2}, a_{3} ; \pm Q \rrbracket:= \begin{cases}\llbracket a_{1}, a_{2}, a_{3} ; Q \rrbracket+\llbracket a_{1}, a_{2}, a_{3} ;-Q \rrbracket & \text { for } Q \neq 0, \\ \llbracket a_{1}, a_{2}, a_{3} ; 0 \rrbracket & \text { for } Q=0 .\end{cases}
$$

$\overline{32}$ Recall that the semicolon in $\llbracket a_{1}, a_{2}, a_{3} ; b \rrbracket$ separating the $U(1)_{R}$ quantum number $b$ from the $S O(7)$ Dynkin labels $a_{1}, a_{2}, a_{3}$ eliminates potential confusion with $\mathcal{N}_{10 d}=1$ supercharacters (6.2).

33 Again, there is a subtlety in applying the above formula to the massless R sector; see the footnote before (4.4). However, this can be fixed easily: one can simply add to it $\frac{1}{2} \prod_{j=1}^{3}\left(y_{j}-y_{j}^{-1}\right)\left(r-r^{-1}\right)$ to get the correct massless character in $\mathrm{R}$ sector. 
Table 11

$\mathcal{N}_{8 d}=1$ multiplets occurring up to mass level six.

\begin{tabular}{ll}
\hline$\alpha^{\prime} m^{2}$ & Representations of $\mathcal{N}_{8 d}=1$ super-Poincaré \\
\hline 1 & $\llbracket 0,0,0 ; 0 \rrbracket$ \\
2 & $\llbracket 0,0,0 ; \pm 2 \rrbracket+\llbracket 1,0,0 ; 0 \rrbracket$ \\
3 & $\llbracket 0,0,0 ; \pm 4 \rrbracket+\llbracket 1,0,0 ; \pm 2 \rrbracket+\llbracket 0,0,1 ; \pm 1 \rrbracket+\llbracket 2,0,0 ; 0 \rrbracket+\llbracket 0,0,0 ; 0 \rrbracket$ \\
4 & $\llbracket 0,0,0 ; \pm 6 \rrbracket+\llbracket 1,0,0 ; \pm 4 \rrbracket+\llbracket 0,0,1 ; \pm 3 \rrbracket+\llbracket 2,0,0 ; \pm 2 \rrbracket+\llbracket 1,0,0 ; \pm 2 \rrbracket+2 \llbracket 0,0,0 ; \pm 2 \rrbracket$ \\
& $+\llbracket 1,0,1 ; \pm 1 \rrbracket+\llbracket 0,0,1 ; \pm 1 \rrbracket+\llbracket 3,0,0 ; 0 \rrbracket+2 \llbracket 1,0,0 ; 0 \rrbracket+\llbracket 0,1,0 ; 0 \rrbracket+\llbracket 0,0,0 ; 0 \rrbracket$ \\
5 & $\llbracket 0,0,0 ; \pm 8 \rrbracket+\llbracket 1,0,0 ; \pm 6 \rrbracket+\llbracket 0,0,1 ; \pm 5 \rrbracket+\llbracket 2,0,0 ; \pm 4 \rrbracket+\llbracket 1,0,0 ; \pm 4 \rrbracket+2 \llbracket 0,0,0 ; \pm 4 \rrbracket$ \\
& $+\llbracket 1,0,1 ; \pm 3 \rrbracket+2 \llbracket 0,0,1 ; \pm 3 \rrbracket+\llbracket 3,0,0 ; \pm 2 \rrbracket+\llbracket 2,0,0 ; \pm 2 \rrbracket+3 \llbracket 1,0,0 ; \pm 2 \rrbracket+2 \llbracket 0,1,0 ; \pm 2 \rrbracket$ \\
& $+\llbracket 0,0,0 ; \pm 2 \rrbracket+\llbracket 2,0,1 ; \pm 1 \rrbracket+2 \llbracket 1,0,1 ; \pm 1 \rrbracket+3 \llbracket 0,0,1 ; \pm 1 \rrbracket+\llbracket 4,0,0 ; 0 \rrbracket+2 \llbracket 2,0,0 ; 0 \rrbracket$ \\
& $+\llbracket 1,1,0 ; 0 \rrbracket+3 \llbracket 1,0,0 ; 0 \rrbracket+\llbracket 0,1,0 ; 0 \rrbracket+\llbracket 0,0,2 ; 0 \rrbracket+4 \llbracket 0,0,0 ; 0 \rrbracket$ \\
& $\llbracket 0,0,0 ; \pm 10 \rrbracket+\llbracket 1,0,0 ; \pm 8 \rrbracket+\llbracket 0,0,1 ; \pm 7 \rrbracket+\llbracket 2,0,0 ; \pm 6 \rrbracket+\llbracket 1,0,0 ; \pm 6 \rrbracket$ \\
& $+2 \llbracket 0,0,0 ; \pm 6 \rrbracket+\llbracket 1,0,1 ; \pm 5 \rrbracket+2 \llbracket 0,0,1 ; \pm 5 \rrbracket+\llbracket 33,0,0 ; \pm 4 \rrbracket+\llbracket 2,0,0 ; \pm 4 \rrbracket$ \\
& $+3 \llbracket 1,0,0 ; \pm 4 \rrbracket+2 \llbracket 0,1,0 ; \pm 4 \rrbracket+2 \llbracket 0,0,0 ; \pm 4 \rrbracket+\llbracket 2,0,1 ; \pm 3 \rrbracket+3 \llbracket 1,0,1 ; \pm 3 \rrbracket+3 \llbracket 0,0,1 ; \pm 3 \rrbracket$ \\
& $+\llbracket 4,0,0 ; \pm 2 \rrbracket+\llbracket 3,0,0 ; \pm 2 \rrbracket+3 \llbracket 2,0,0 ; \pm 2 \rrbracket+2 \llbracket 1,1,0 ; \pm 2 \rrbracket+5 \llbracket 1,0,0 ; \pm 2 \rrbracket+\llbracket 0,1,0 ; \pm 2 \rrbracket$ \\
& $+2 \llbracket 0,0,2 ; \pm 2 \rrbracket+4 \llbracket 0,0,0 ; \pm 2 \rrbracket+\llbracket 3,0,1 ; \pm 1 \rrbracket+2 \llbracket 2,0,1 ; \pm 1 \rrbracket+4 \llbracket 1,0,1 ; \pm 1 \rrbracket$ \\
& $+\llbracket 0,1,1 ; \pm 1 \rrbracket+5 \llbracket 0,0,1 ; \pm 1 \rrbracket+\llbracket 5,0,0 ; 0 \rrbracket+2 \llbracket 3,0,0 ; 0 \rrbracket+\llbracket 2,1,0 ; 0 \rrbracket+4 \llbracket 2,0,0 ; 0 \rrbracket$ \\
& $+\llbracket 1,1,0 ; 0 \rrbracket+\llbracket 1,0,2 ; 0 \rrbracket+5 \llbracket 1,0,0 ; 0 \rrbracket+5 \llbracket 0,1,0 ; 0 \rrbracket+\llbracket 0,0,2 ; 0 \rrbracket+3 \llbracket 0,0,0 ; 0 \rrbracket$ \\
&
\end{tabular}

The supermultiplets up to level six are listed in Table 11, some of their scattering amplitudes are discussed in $[43,44]$. The branching process obviously increases the number and diversity of multiplets compared to the ten-dimensional analogue, cf. Table 8 . This is why we do not repeat the higher level analysis carried out for the $d=10$ ancestor in dimensionally reduced settings.

Note that this partition function can also be obtained by branching the $S O(9)$ representations appearing in the $\mathcal{N}_{10 d}=1$ partition function (6.4) into $S O(7) \times U(1)_{R}$ representations. In terms of characters, one simply maps $S O(9)$ fugacities into $S O(7) \times U(1)_{R}$ fugacities; a possible fugacity map is as follows:

$$
z_{1}=y_{1}, \quad z_{2}=y_{2}, \quad z_{3}=y_{3}, \quad z_{4}=s,
$$

where $z_{1}, \ldots, z_{4}$ are fugacities of $S O(9), y_{1}, y_{2}, y_{3}$ are fugacities of $S O(7)$ and $s$ is a fugacity of $U(1)_{R}$. For example,

$$
\begin{aligned}
{[1,0,0,0]_{z} } & =1+\frac{1}{z_{1}^{2}}+z_{1}^{2}+\frac{1}{z_{2}^{2}}+z_{2}^{2}+\frac{1}{z_{3}^{2}}+z_{3}^{2}+\frac{1}{z_{4}^{2}}+z_{4}^{2} \\
& =1+\frac{1}{y_{1}^{2}}+y_{1}^{2}+\frac{1}{y_{2}^{2}}+y_{2}^{2}+\frac{1}{y_{3}^{2}}+y_{3}^{2}+\frac{1}{s^{2}}+s^{2} \\
& =[1,0,0 ; 0]_{\boldsymbol{y} ; s}+[0,0,0 ;+2]_{\boldsymbol{y} ; s}+[0,0,0 ;-2]_{\boldsymbol{y} ; s},
\end{aligned}
$$

where the notation $\left[b_{1}, b_{2}, b_{3} ; Q\right]$ denotes the $S O(7) \times U(1)_{R}$ representation.

\subsection{Six-dimensional $\mathcal{N}_{6 d}=(1,1)$ spectra}

Six-dimensional Type I compactifications with sixteen supercharges are said to possess $\mathcal{N}_{6 d}=$ $(1,1)$ SUSY. The spacetime symmetry branches to $S O(9) \rightarrow S O(5) \times S O(4)_{R}$, i.e. two Cartan generators of ten-dimensional Lorentz group take the role of $\mathrm{R}$ symmetry generators probing fugacities $r_{1}, r_{2}$ of $S O(4)_{R} \cong S U(2)_{R} \times S U(2)_{R}$. The fundamental supermultiplet of the $\mathcal{N}_{6 d}=$ $(1,1)$ super-Poincaré group has the following $S O(5) \times S U(2)_{R} \times S U(2)_{R}$ particle content: 


$$
\begin{aligned}
Z\left(\mathcal{N}_{6 d}=(1,1)\right):= & {[2,0] \cdot[0,0]_{R}+[0,2] \cdot[0,0]_{R} } \\
& +[0,2] \cdot[1,1]_{R}+[1,0] \cdot[1,1]_{R} \\
& +[1,0] \cdot\left([2,0]_{R}+[0,2]_{R}\right)+[0,0] \cdot[2,2]_{R} \\
& +[0,0] \cdot[1,1]_{R}+[0,0] \cdot[0,0]_{R} \\
& +[1,1] \cdot\left([1,0]_{R}+[0,1]_{R}\right) \\
& +[0,1] \cdot\left([2,1]_{R}+[1,2]_{R}+[1,0]_{R}+[0,1]_{R}\right) .
\end{aligned}
$$

Note that the R-symmetry characters $[\ldots]_{R}$ carry a subscript to avoid confusion with the Lorentz symmetry of identical rank.

The most general multiplet follows from (6.48) by taking tensor products with $S O(5) \times$ $S U(2)_{R} \times S U(2)_{R}$ representations, this leads to the supercharacter

$$
\llbracket a_{1}, a_{2} ; b_{1}, b_{2} \rrbracket:=Z\left(\mathcal{N}_{6 d}=(1,1)\right) \cdot\left[a_{1}, a_{2}\right] \cdot\left[b_{1}, b_{2}\right]_{R} .
$$

The six-dimensional partition function is obtained from its ten-dimensional ancestor (6.3) by singling out two internal factor $\chi_{\mathrm{NS}, \mathrm{R}}^{S O(3)}$ within $\chi_{\mathrm{NS}, \mathrm{R}}^{S O(9)}(\boldsymbol{y})=\prod_{k=1}^{4} \chi_{\mathrm{NS}, \mathrm{R}}^{S O(3)}\left(y_{k}\right)$ and reinterpreting their second argument as an $R$-symmetry fugacity:

$$
\begin{aligned}
& \chi^{\mathcal{N}_{6 d}=(1,1)}(q ; \boldsymbol{y}, \boldsymbol{r})=\left.\chi_{\mathrm{NS}}^{\mathcal{N}_{6 d}=(1,1)}\right|_{\mathrm{GSO}}(q ; \boldsymbol{y}, \boldsymbol{r})+\left.\chi_{\mathrm{R}}^{\mathcal{N}_{6 d}=(1,1)}\right|_{\mathrm{GSO}}(q ; \boldsymbol{y}, \boldsymbol{r}), \\
& \left.\chi_{\mathrm{NS}}^{\mathcal{N}_{6 d}=(1,1)}\right|_{\mathrm{GSO}}(q ; \boldsymbol{y}, \boldsymbol{r}) \\
& \quad=\frac{1}{2} q^{-\frac{1}{2}}\left[\chi_{\mathrm{NS}}^{S O(5)}(q ; \boldsymbol{y}) \chi_{\mathrm{NS}}^{S O(5)}(q ; \boldsymbol{r})-\chi_{\mathrm{NS}}^{S O(5)}\left(e^{2 \pi i} q ; \boldsymbol{y}\right) \chi_{\mathrm{NS}}^{S O(5)}\left(e^{2 \pi i} q ; \boldsymbol{r}\right)\right], \\
& \left.\chi_{\mathrm{R}}^{\mathcal{N}_{6 d}=(1,1)}\right|_{\mathrm{GSO}}(q ; \boldsymbol{y}, \boldsymbol{r})=\frac{1}{2} \chi_{\mathrm{R}}^{S O(5)}(q ; \boldsymbol{y}) \chi_{\mathrm{R}}^{S O(5)}(q ; \boldsymbol{r}) .
\end{aligned}
$$

Its $q$ expansion starts like $\mathrm{e}^{34}$

$$
\begin{aligned}
& \chi^{\mathcal{N}_{6 d}=(1,1)}(q ; \boldsymbol{y}, \boldsymbol{r}) \\
& =\underbrace{\left(\sum_{j=1}^{2}\left(y_{j}^{2}+y_{j}^{-2}\right)+\sum_{j=1}^{2}\left(r_{j}^{2}+r_{j}^{-2}\right)+\frac{1}{2} \prod_{j=1}^{2}\left(y_{j}+y_{j}^{-1}\right) \prod_{j=1}^{2}\left(r_{j}+r_{j}^{-1}\right)\right) q^{0}} \\
& 16 \text { massless states } \\
& +\underbrace{\llbracket 0,0 ; 0,0 \rrbracket q}_{256 \text { states at level } 1}+\underbrace{(\llbracket 0,0 ; 1,1 \rrbracket+\llbracket 1,0 ; 0,0 \rrbracket) q^{2}}_{2304 \text { states at level } 2} \\
& +(\llbracket 0,0 ; 2,2 \rrbracket+\llbracket 1,0 ; 1,1 \rrbracket+\llbracket 0,1 ; 1,0 \rrbracket \\
& +\llbracket 0,1 ; 0,1 \rrbracket+\llbracket 2,0 ; 0,0 \rrbracket+\llbracket 0,0 ; 0,0 \rrbracket) q^{3}+\mathcal{O}\left(q^{4}\right),
\end{aligned}
$$

and supermultiplets at higher levels $\leqslant 5$ are listed in Table 12.

Note that this partition function can also be obtained by branching the $S O(9)$ representations appearing in the $\mathcal{N}_{10 d}=1$ partition function (6.4) into $S O(5) \times S U(2)_{R} \times S U(2)_{R}$ representations. In terms of characters, one simply maps $S O(9)$ fugacities into $S O(5) \times S U(2)_{R} \times S U(2)_{R}$ fugacities; a possible fugacity map is as follows:

\footnotetext{
34 Again, there is a subtlety in applying the above formula to the massless R sector; see the footnote before (4.4). However, this can be fixed easily: one can simply add to it $\frac{1}{2} \prod_{j=1}^{2}\left(y_{j}-y_{j}^{-1}\right) \prod_{j=1}^{2}\left(r_{j}-r_{j}^{-1}\right)$ to get the correct massless character in $\mathrm{R}$ sector.
} 
Table 12

$\mathcal{N}_{6 d}=(1,1)$ multiplets occurring up to mass level five.

\begin{tabular}{|c|c|}
\hline$\alpha^{\prime} m^{2}$ & Representations of $\mathcal{N}_{6 d}=(1,1)$ super-Poincaré \\
\hline 1 & $\llbracket 0,0 ; 0,0 \rrbracket$ \\
\hline 2 & $\llbracket 0,0 ; 1,1 \rrbracket+\llbracket 1,0 ; 0,0 \rrbracket$ \\
\hline 3 & $\llbracket 0,0 ; 2,2 \rrbracket+\llbracket 1,0 ; 1,1 \rrbracket+\llbracket 0,1 ; 1,0 \rrbracket+\llbracket 0,1 ; 0,1 \rrbracket+\llbracket 2,0 ; 0,0 \rrbracket+\llbracket 0,0 ; 0,0 \rrbracket$ \\
\hline 4 & $\begin{array}{l}\llbracket 0,0 ; 3,3 \rrbracket+\llbracket 1,0 ; 2,2 \rrbracket+\llbracket 0,1 ; 2,1 \rrbracket+\llbracket 0,0 ; 2,0 \rrbracket+\llbracket 0,1 ; 1,2 \rrbracket+\llbracket 2,0 ; 1,1 \rrbracket+\llbracket 1,0 ; 1,1 \rrbracket \\
\quad+2 \llbracket 0,0 ; 1,1 \rrbracket+\llbracket 1,1 ; 1,0 \rrbracket+\llbracket 0,1 ; 1,0 \rrbracket+\llbracket 0,0 ; 0,2 \rrbracket+\llbracket 1,1 ; 0,1 \rrbracket+\llbracket 0,1 ; 0,1 \rrbracket+\llbracket 3,0 ; 0,0 \rrbracket \\
\quad+2 \llbracket 1,0 ; 0,0 \rrbracket+\llbracket 0,2 ; 0,0 \rrbracket\end{array}$ \\
\hline 5 & $\begin{array}{l}\llbracket 0,0 ; 4,4 \rrbracket+\llbracket 1,0 ; 3,3 \rrbracket+\llbracket 0,1 ; 3,2 \rrbracket+\llbracket 0,0 ; 3,1 \rrbracket+\llbracket 0,1 ; 2,3 \rrbracket+\llbracket 2,0 ; 2,2 \rrbracket+\llbracket 1,0 ; 2,2 \rrbracket \\
\quad+2 \llbracket 0,0 ; 2,2 \rrbracket+\llbracket 1,1 ; 2,1 \rrbracket+2 \llbracket 0,1 ; 2,1 \rrbracket+2 \llbracket 1,0 ; 2,0 \rrbracket+\llbracket 0,0 ; 2,0 \rrbracket+\llbracket 0,0 ; 1,3 \rrbracket+\llbracket 1,1 ; 1,2 \rrbracket \\
\quad+2 \llbracket 0,1 ; 1,2 \rrbracket+\llbracket 3,0 ; 1,1 \rrbracket+\llbracket 2,0 ; 1,1 \rrbracket+3 \llbracket 1,0 ; 1,1 \rrbracket+2 \llbracket 0,2 ; 1,1 \rrbracket+2 \llbracket 0,0 ; 1,1 \rrbracket+\llbracket 2,1 ; 1,0 \rrbracket \\
\quad+2 \llbracket 1,1 ; 1,0 \rrbracket+3 \llbracket 0,1 ; 1,0 \rrbracket+2 \llbracket 1,0 ; 0,2 \rrbracket+\llbracket 0,0 ; 0,2 \rrbracket+\llbracket 2,1 ; 0,1 \rrbracket+2 \llbracket 1,1 ; 0,1 \rrbracket+3 \llbracket 0,1 ; 0,1 \rrbracket \\
\quad+\llbracket 4,0 ; 0,0 \rrbracket+2 \llbracket 2,0 ; 0,0 \rrbracket+\llbracket 1,2 ; 0,0 \rrbracket+\llbracket 1,0 ; 0,0 \rrbracket+2 \llbracket 0,2 ; 0,0 \rrbracket+3 \llbracket 0,0 ; 0,0 \rrbracket\end{array}$ \\
\hline
\end{tabular}

$$
z_{1}=y_{1}, \quad z_{2}=y_{2}, \quad z_{3}=r_{1} r_{2}, \quad z_{4}=r_{1} r_{2}^{-1},
$$

where $z_{1}, \ldots, z_{4}$ are fugacities of $S O(9), y_{1}, y_{2}$ are fugacities of $S O(5)$, and $r_{1}, r_{2}$ are fugacities for the two $S U(2)_{R}$ factors. For example,

$$
\begin{aligned}
{[1,0,0,0]_{z} } & =1+\frac{1}{z_{1}^{2}}+z_{1}^{2}+\frac{1}{z_{2}^{2}}+z_{2}^{2}+\frac{1}{z_{3}^{2}}+z_{3}^{2}+\frac{1}{z_{4}^{2}}+z_{4}^{2} \\
& =1+\frac{1}{y_{1}^{2}}+y_{1}^{2}+\frac{1}{y_{2}^{2}}+y_{2}^{2}+\left(r_{1}+r_{1}^{-1}\right)\left(r_{2}+r_{2}^{-1}\right) \\
& =[1,0 ; 0,0]_{\boldsymbol{y} ; \boldsymbol{r}}+[0,0 ; 1,1]_{\boldsymbol{y} ; \boldsymbol{r}},
\end{aligned}
$$

where the notation $\left[a_{1}, a_{2} ; b_{1}, b_{2}\right]$ denotes the $S O(5) \times S U(2)_{R} \times S U(2)_{R}$ representation.

\subsection{Four-dimensional $\mathcal{N}_{4 d}=4$ spectra}

Finally, four-dimensional theories with maximal $\mathcal{N}_{4 d}=4$ SUSY follow from the tendimensional ancestor through compactification on $T^{6}$. The internal rotation group is identified with the R symmetry $S O(6)_{R}$, its characters are denoted by $\left[b_{1}, b_{2}, b_{3}\right]_{R}$. The universal partition function decomposes into characters of the $\mathcal{N}_{4 d}=4$ super-Poincaré algebra, the fundamental one being

$$
\begin{aligned}
Z\left(\mathcal{N}_{4 d}=4\right)= & {[0]\left([0,0,2]_{R}+[0,2,0]_{R}+[2,0,0]_{R}+2\right) } \\
& +[2][0,1,1]_{R}+2[2][1,0,0]_{R}+[4] \\
& +[1]\left([0,0,1]_{R}+[0,1,0]_{R}+[1,0,1]_{R}+[1,1,0]_{R}\right) \\
& +[3]\left([0,0,1]_{R}+[0,1,0]_{R}\right) .
\end{aligned}
$$

Any other supermultiplet follows by taking a tensor product of (6.54) with the $S O(3) \times S O(6)_{R}$ representation $[n]\left[b_{1}, b_{2}, b_{3}\right]_{R}$ of the Clifford vacuum,

$$
\llbracket n ; b_{1}, b_{2}, b_{3} \rrbracket:=Z\left(\mathcal{N}_{4 d}=4\right) \cdot[n]\left[b_{1}, b_{2}, b_{3}\right]_{R} .
$$

The four-dimensional partition function is obtained through the usual procedure from the tendimensional ancestor (6.3), this time we have to interpret three factors of $\chi_{\mathrm{NS}, \mathrm{R}}^{S O(3)}$ as carrying R-symmetry fugacities $r_{j}$ : 


$$
\begin{aligned}
& \chi^{\mathcal{N}_{4 d}=4}(q ; y, \boldsymbol{r})=\left.\chi_{\mathrm{NS}}^{\mathcal{N}_{4 d}=4}\right|_{\mathrm{GSO}}(q ; y, \boldsymbol{r})+\left.\chi_{\mathrm{R}}^{\mathcal{N}_{4 d}=4}\right|_{\mathrm{GSO}}(q ; y, \boldsymbol{r}), \\
& \left.\chi_{\mathrm{NS}}^{\mathcal{N}_{4 d}=4}\right|_{\mathrm{GSO}}(q ; y, \boldsymbol{r})=\frac{1}{2} q^{-\frac{1}{2}}\left[\chi_{\mathrm{NS}}^{S O(3)}(q ; y) \chi_{\mathrm{NS}}^{S O(7)}(q ; \boldsymbol{r})\right. \\
& \left.-\chi_{\mathrm{NS}}^{S O(3)}\left(e^{2 \pi i} q ; y\right) \chi_{\mathrm{NS}}^{S O(7)}\left(e^{2 \pi i} q ; \boldsymbol{r}\right)\right] \\
& \left.\chi_{\mathrm{R}}^{\mathcal{N}_{4 d}=4}\right|_{\mathrm{GSO}}(q ; y, \boldsymbol{r})=\frac{1}{2} \chi_{\mathrm{R}}^{S O(3)}(q ; y) \chi_{\mathrm{R}}^{S O(7)}(q ; \boldsymbol{r}) .
\end{aligned}
$$

The power series in $q$ starts with ${ }^{35}$

$$
\begin{aligned}
\chi^{\mathcal{N}_{4 d}}=4 & \left(q ; y, r_{j}\right) \\
= & \underbrace{\left(y^{2}+y^{-2}+\sum_{j=1}^{3}\left(r_{j}^{2}+r_{j}^{-2}\right)+\frac{1}{2}[1]_{y} \prod_{j=1}^{3}\left(r_{j}+r_{j}^{-1}\right)\right) q^{0}}_{16 \text { massless states }}+\underbrace{\llbracket 0 ; 0,0,0 \rrbracket q}_{256 \text { states at level } 1} \\
& +\underbrace{(\llbracket 0 ; 1,0,0 \rrbracket+\llbracket 2 ; 0,0,0 \rrbracket) q^{2}}_{2304 \text { states at level } 2}+(\llbracket 0 ; 0,0,0 \rrbracket+\llbracket 0 ; 2,0,0 \rrbracket+\llbracket 1 ; 0,0,1 \rrbracket \\
& +\llbracket 1 ; 0,1,0 \rrbracket+\llbracket 2 ; 1,0,0 \rrbracket+\llbracket 4 ; 0,0,0 \rrbracket) q^{3}+\mathcal{O}\left(q^{4}\right),
\end{aligned}
$$

the coefficients of $q^{4}$ and $q^{5}$ can be found in Table 13. The explicit vertex operators from the first level are listed in Section 4 of [33].

Note that this partition function can also be obtained by branching the $S O(9)$ representations appearing in the $\mathcal{N}_{10 d}=1$ partition function (6.4) into $S O(3) \times S O(6)_{R}$ representations. In terms of characters, one simply maps $S O(9)$ fugacities into $S O(3) \times S O(6)_{R}$ fugacities; a possible fugacity map is as follows:

$$
z_{1}=r_{1}, \quad z_{2}=r_{2}, \quad z_{3}=r_{3}, \quad z_{4}=y,
$$

where $z_{1}, \ldots, z_{4}$ are fugacities of $S O(9), r_{1}, r_{2}, r_{3}$ are fugacities of $S O(6)_{R}$ and $y$ is a fugacity of $S O(3)$. For example,

$$
\begin{aligned}
{[1,0,0,0]_{z} } & =1+\frac{1}{z_{1}^{2}}+z_{1}^{2}+\frac{1}{z_{2}^{2}}+r_{2}^{2}+\frac{1}{z_{3}^{2}}+z_{3}^{2}+\frac{1}{z_{4}^{2}}+z_{4}^{2} \\
& =\frac{1}{r_{1}^{2}}+r_{1}^{2}+\frac{1}{r_{2}^{2}}+r_{2}^{2}+\frac{1}{r_{3}^{2}}+r_{3}^{2}+\left(1+\frac{1}{y^{2}}+y^{2}\right) \\
& =[0 ; 1,0,0]_{\boldsymbol{r} ; y}+[2 ; 0,0,0]_{\boldsymbol{r} ; y},
\end{aligned}
$$

where the notation $\left[a ; b_{1}, b_{2}, b_{3}\right]$ denotes the $S O(3) \times S O(6)_{R}$ representation for which the $S O(3)$ representation is $[a]$ and $S O(6)_{R}$ representation is $\left[b_{1}, b_{2}, b_{3}\right]_{R}$.

\section{Conclusion}

We have investigated model independent superstring states common to all Type I compactifications that preserve $\mathcal{N}_{4 d}=1$ and $\mathcal{N}_{6 d}=(1,0)$ SUSY, respectively, and identified the underlying

\footnotetext{
35 Again, there is a subtlety in applying the above formula to the massless R sector; see the footnote before (4.4). However, this can be fixed easily: one can simply add to it $\frac{1}{2}\left(y-y^{-1}\right) \prod_{j=1}^{3}\left(r_{j}-r_{j}^{-1}\right)$ to get the correct massless character in $\mathrm{R}$ sector.
} 
Table 13

$\mathcal{N}_{4 d}=4$ multiplets occurring up to mass level 5 .

\begin{tabular}{ll}
\hline$\alpha^{\prime} m^{2}$ & Representations of $\mathcal{N}_{4 d}=4$ super-Poincaré \\
\hline 1 & $\llbracket 0 ; 0,0,0 \rrbracket$ \\
2 & $\llbracket 0 ; 1,0,0 \rrbracket+\llbracket 2 ; 0,0,0 \rrbracket$ \\
3 & $\llbracket 0 ; 0,0,0 \rrbracket+\llbracket 0 ; 2,0,0 \rrbracket+\llbracket 1 ; 0,0,1 \rrbracket+\llbracket 1 ; 0,1,0 \rrbracket+\llbracket 2 ; 1,0,0 \rrbracket+\llbracket 4 ; 0,0,0 \rrbracket$ \\
4 & $\llbracket 0 ; 0,1,1 \rrbracket+2 \llbracket 0 ; 1,0,0 \rrbracket+\llbracket 0 ; 3,0,0 \rrbracket+\llbracket 1 ; 0,0,1 \rrbracket+\llbracket 1 ; 0,1,0 \rrbracket+\llbracket 1 ; 1,0,1 \rrbracket+\llbracket 1 ; 1,1,0 \rrbracket$ \\
& $+3 \llbracket 2 ; 0,0,0 \rrbracket+\llbracket 2 ; 1,0,0 \rrbracket+\llbracket 2 ; 2,0,0 \rrbracket+\llbracket 3 ; 0,0,1 \rrbracket+\llbracket 3 ; 0,1,0 \rrbracket$ \\
& $+\llbracket 4 ; 1,0,0 \rrbracket+\llbracket 6 ; 0,0,0 \rrbracket$ \\
4 & \\
& \\
5 & \\
& $+2 \llbracket 0 ; 0,0 \rrbracket+\llbracket 0 ; 0,0,2 \rrbracket+\llbracket 0 ; 0,1,1 \rrbracket+\llbracket 0 ; 0,2,0 \rrbracket+\llbracket 0 ; 1,0,0 \rrbracket+\llbracket 0 ; 1,1,1 \rrbracket$ \\
& $+2 \llbracket 1 ; 1,1,0 \rrbracket+\llbracket 1 ; 2,0,1 \rrbracket+\llbracket 1 ; 2,1,0 \rrbracket+2 \llbracket 2 ; 0,0,0 \rrbracket+2 \llbracket 2 ; 0,1,1 \rrbracket+5 \llbracket 2 ; 1,0,0 \rrbracket$ \\
& $+\llbracket 2 ; 2,0,0 \rrbracket+\llbracket 2 ; 3,0,0 \rrbracket+2 \llbracket 3 ; 0,0,1 \rrbracket+2 \llbracket 3 ; 0,1,0 \rrbracket+\llbracket 3 ; 1,0,1 \rrbracket+\llbracket 3 ; 1,1,0 \rrbracket$ \\
& $+3 \llbracket 4 ; 0,0,0 \rrbracket+\llbracket 4 ; 1,0,0 \rrbracket+\llbracket 4 ; 2,0,0 \rrbracket+\llbracket 5 ; 0,0,1 \rrbracket+\llbracket 5 ; 0,1,0 \rrbracket$ \\
& $+\llbracket 6 ; 1,0,0 \rrbracket+\llbracket 8 ; 0,0,0 \rrbracket$
\end{tabular}

super-Poincaré multiplets at individual mass levels. Part of our results are the associated unrefined partition functions together with their asymptotics for large mass levels, see (4.8)-(4.17) and (5.5)-(5.12). The refined versions of the universal partition functions are given by (4.4) and (5.3) and rewritten in terms of super-Poincaré characters in (4.39), (4.61), (4.62), (5.23) and (5.41). Moreover, we have presented dimensional reductions of the universal $\mathcal{N}_{6 d}=(1,0)$ and $\mathcal{N}_{10 d}=1$ spectra to even dimensions $d \geqslant 4$ in Sections 5.5, 6.3, 6.4 and 6.5.

Multiplicity generating functions for individual supermultiplets tend to stabilize in the regime where the spin $j$ (or more generally the first $S O(d-1)$ Dynkin label) is comparable to the mass level $M=\alpha^{\prime} m^{2}$. More specifically, the validity for the stable pattern roughly ranges between $\frac{1}{2}\left(M-M_{0}\right) \lesssim j \lesssim M-M_{0}$ where the offset $M_{0}$ depends on the remaining super-Poincaré quantum numbers of the multiplets beyond the spin. In the mathematically most tractable $\mathcal{N}_{4 d}=1$ case, we have derived closed formulae (4.63) and (4.64) for the leading Regge trajectory. In the highest-dimensional scenarios with given number of supercharges $-\mathcal{N}_{4 d}=1, \mathcal{N}_{6 d}=(1,0)$ and $\mathcal{N}_{10 d}=1$ - we extracted both leading and subleading Regge trajectories from explicitly computed multiplicities up to level $\alpha^{\prime} m^{2}=25$, see Sections 4.5, 5.4 and 6.2.

Identifying the super-Poincaré covariant spectrum in scenarios with different numbers of supercharges provides a significant step towards a better understanding of the string S-matrix. As pointed out in [43], cubic tree level vertices among all the massive states are the seeds for superstring amplitudes of higher multiplicity and genus. The results of this work appear inspiring to push this programme further, using on-shell superspace techniques in various dimensions $[38,42]$. Refined partition functions as computed here serve as generating functions for helicity supertraces [45] which allow to disentangle contribution of individual supermultiplets to loop amplitudes.

Extending flat space results as presented in this work to curved spacetime provides an exciting direction of further research. Anti-de-Sitter backgrounds are of particular interest in view of their conjectured correspondence to conformal field theories [46,47]. For instance, the model independent higher spin string spectrum at the first massive level in $A d S_{3} \times S^{3}$ compactifications with pure NSNS background has been pioneered in [48]. This is a motivating starting point towards generalizations to non-zero RR flux and superstrings in $A d S_{5} \times S^{5}$, see [49] for a review. Also, we would like to mention Ref. [50] which extracts information on the $A d S_{5} \times S^{5}$ Kaluza Klein excitations from the $\mathcal{N}_{10 d}=1$ flat space spectrum, in particular from its large spin regime investigated in detail here. Finally it would be also very interesting to explore the extended sym- 
metry structure of the universal higher spin states in supersymmetric string compactifications, in analogy to the $\mathcal{W}_{N}$-symmetries in three-dimensional higher spin theories on $A d S_{3}$ [51-53].

\section{Acknowledgements}

We are very grateful to Stefan Hohenegger and Roberto Volpato for a number of useful discussions. N.M. would like to express his gratitude towards the following institutes and collaborators for the hospitality during the completion of this project: Max-Planck-Instituts für Gravitationsphysik (Albert-Einstein-Institut), Humboldt-Universität zu Berlin, Imperial College London, 2012 Simons Summer Workshop at Simons Center for Geometry and Physics; Matthias Staudacher, Amihay Hanany, Theerasak Mingarcha and Aroonroj Mekareeya. O.S. is grateful to the Max Planck Institute Munich for hospitality during various stages of the project and to Rutger Boels, Wan-Zhe Feng, Stephan Stieberger and Tomasz Taylor for collaboration on related projects. The work of N.M. is supported by a research grant of the Max Planck Society.

\section{Appendix A. Notation and conventions}

Unless stated otherwise, the following notation and conventions will be used throughout the paper.

\section{A.1. Group and representation theoretic objects}

- The plethystic exponential of a multivariate function $f\left(t_{1}, \ldots, t_{n}\right)$ that vanishes at the origin, $f(0, \ldots, 0)=0$, is defined as

$$
\operatorname{PE}\left[f\left(t_{1}, \ldots, t_{n}\right)\right]=\exp \left(\sum_{k=1}^{\infty} \frac{1}{k} f\left(t_{1}^{k}, \ldots, t_{n}^{k}\right)\right) .
$$

The fermionic plethystic exponential is defined by

$$
\operatorname{PE}_{F}\left[f\left(t_{1}, \ldots, t_{n}\right)\right]=\exp \left(\sum_{k=1}^{\infty} \frac{(-1)^{k+1}}{k} f\left(t_{1}^{k}, \ldots, t_{n}^{k}\right)\right) .
$$

- An irreducible representation of a simple group $G$ can be denoted by its highest weight vector.

- With respect to a basis consisting of the fundamental weights (the $\omega$-basis), we write the highest vector as $\left[a_{1}, \ldots, a_{r}\right]$ with $r=\operatorname{rank} G$. This is also known as the Dynkin label.

- With respect to a basis of the dual Cartan subalgebra (the $e$-basis), we write the highest vector as $\left(\lambda_{1}, \ldots, \lambda_{r}\right)$.

- Note that we use the round brackets to distinguish the latter from the former for which the square brackets are used.

- For $S O(2 n+1)$, the label $\left[a_{1}, a_{2}, \ldots, a_{n}\right]$ is related to the label $\left(\lambda_{1}, \lambda_{2}, \ldots, \lambda_{n}\right)$ by the formula

$$
\begin{aligned}
& \lambda_{i}=a_{i}+a_{i+1}+\cdots+a_{n-1}+\frac{1}{2} a_{n}, \quad 1 \leqslant i \leqslant n-1, \\
& \lambda_{n}=\frac{1}{2} a_{n},
\end{aligned}
$$


or equivalently

$$
\begin{aligned}
& a_{i}=\lambda_{i}-\lambda_{i+1}, \quad 1 \leqslant i \leqslant n-1, \\
& a_{n}=2 \lambda_{n} .
\end{aligned}
$$

- Note that a representation is uniquely specified by its character. We use the notation $\left[a_{1}, a_{2}, \ldots, a_{r}\right]_{y}$ (resp. $\left.\left(\lambda_{1}, \ldots, \lambda_{r}\right)_{y}\right)$ to denote the character of the representation $\left[a_{1}, a_{2}, \ldots, a_{r}\right]$ (resp. $\left.\left(\lambda_{1}, \ldots, \lambda_{r}\right)\right)$ written in terms of the variables $\boldsymbol{y}=\left(y_{1}, \ldots, y_{r}\right)$. Whenever there is no potential confusion, we drop the subscript $\boldsymbol{y}$ to avoid cluttered notation.

- A representation of a product group $G_{1} \times G_{2}$ is denoted by $\left[a_{1}, \ldots, a_{r_{1}} ; b_{1}, b_{2}, \ldots, b_{r_{2}}\right]$ where $\left[a_{1}, \ldots, a_{r_{1}}\right]$ is an irreducible representation of $G_{1}$ and $\left[b_{1}, \ldots, b_{r_{2}}\right]$ is that of $G_{2}$. We use a semi-colon (;) to separate each representation.

- We use the notation $[n]$ to denote the $(n+1)$-dimensional irreducible representation of $S U(2)$ and $S O(3)$. Its character is given by

$$
[n]_{y}=\sum_{k=-n / 2}^{+n / 2} y^{2 k}
$$

- The character of the vector representation of $S O(2 n+1)$, with $n>1$, is taken to be

$$
(1,0, \ldots, 0)_{y}=[1,0, \ldots, 0]_{y}=1+\sum_{k=1}^{n}\left(y_{k}^{2}+y_{k}^{-2}\right) .
$$

In general, the character of the irreducible representation $\left(\lambda_{1}, \ldots, \lambda_{n}\right)$ of $S O(2 n+1)$ is given by the Weyl character formula:

$$
\left(\lambda_{1}, \ldots, \lambda_{n}\right)_{y}=\frac{\operatorname{det}\left(y_{j}^{2\left(\lambda_{i}+n-i+\frac{1}{2}\right)}-y_{j}^{-2\left(\lambda_{i}+n-i+\frac{1}{2}\right)}\right)_{i, j=1}^{n}}{\operatorname{det}\left(y_{j}^{2\left(n-i+\frac{1}{2}\right)}-y_{j}^{-2\left(n-i+\frac{1}{2}\right)}\right)_{i, j=1}^{n}} .
$$

- The choice of the character in (A.6) has a great advantage: One can relate the character of the vector representation of $S O(2 n+1)$ to that of the vector representation of $S O(3)$ in a simple way:

$$
[1,0, \ldots, 0]_{y}^{S O(2 n+1)}=\sum_{k=1}^{n}[2]_{y_{k}}^{S O(3)}-(n-1) .
$$

As we shall see in subsequent sections, this helps simplify a number of computations.

- The Haar measures of $S O(3)$ and $S U(2)$ are taken to be

$$
\int \mathrm{d} \mu_{S O(3)}(y)=\int \mathrm{d} \mu_{S U(2)}(y)=\frac{1}{2} \frac{1}{2 \pi i} \oint_{|y|=1} \frac{\mathrm{d} y}{y}\left(1-y^{2}\right)\left(1-y^{-2}\right) .
$$

In general, the Haar measure for $S O(2 n+1)$ can be written as

$$
\int \mathrm{d} \mu_{S O(2 n+1)}(\boldsymbol{y})=\int \mathrm{d} \mu_{S O(3)}\left(y_{1}\right) \cdots \int \mathrm{d} \mu_{S O(3)}\left(y_{n}\right) \rho(\boldsymbol{y}),
$$

where

$$
\rho(\boldsymbol{y})=\frac{1}{n !} \prod_{1 \leqslant i<j \leqslant n}\left(1-y_{i}^{2} y_{j}^{2}\right)\left(1-y_{i}^{-2} y_{j}^{-2}\right)\left(1-y_{i}^{2} y_{j}^{-2}\right)\left(1-y_{i}^{-2} y_{j}^{2}\right) .
$$




\section{A.1.1. Special functions}

- The $q$-Pochhammer symbols are defined as

$$
(a ; q)_{n}=\prod_{k=0}^{n-1}\left(1-a q^{k}\right), \quad(a ; q)_{\infty}=\prod_{k=0}^{\infty}\left(1-a q^{k}\right) .
$$

- Our conventions for the Dedekind eta and the Jacobi theta functions are ${ }^{36}$

$$
\begin{aligned}
& \eta(q)=q^{\frac{1}{24}} \prod_{n=1}^{\infty}\left(1-q^{n}\right)=q^{\frac{1}{24}}(q ; q)_{\infty}, \\
& \vartheta_{1}(y, q)=-i q^{\frac{1}{8}}\left(y^{\frac{1}{2}}-y^{-\frac{1}{2}}\right) \prod_{n=1}^{\infty}\left(1-q^{n}\right)\left(1-y q^{n}\right)\left(1-y^{-1} q^{n}\right), \\
& \vartheta_{2}(y, q)=q^{\frac{1}{8}}\left(y^{\frac{1}{2}}+y^{-\frac{1}{2}}\right) \prod_{n=1}^{\infty}\left(1-q^{n}\right)\left(1+y q^{n}\right)\left(1+y^{-1} q^{n}\right), \\
& \vartheta_{3}(y, q)=\prod_{n=1}^{\infty}\left(1-q^{n}\right)\left(1+y q^{n-1 / 2}\right)\left(1+y^{-1} q^{n-1 / 2}\right), \\
& \vartheta_{4}(y, q)=\prod_{n=1}^{\infty}\left(1-q^{n}\right)\left(1-y q^{n-1 / 2}\right)\left(1-y^{-1} q^{n-1 / 2}\right) .
\end{aligned}
$$

In terms of an infinite sum, the Jacobi theta functions can be written as

$$
\vartheta\left[\begin{array}{l}
a \\
b
\end{array}\right](y, q)=\sum_{m \in \mathbb{Z}} q^{\frac{1}{2}(m-a / 2)^{2}}\left(e^{-i \pi b} y\right)^{(m-a / 2)},
$$

where

$$
\vartheta_{1}=\vartheta\left[\begin{array}{l}
1 \\
1
\end{array}\right], \quad \vartheta_{2}=\vartheta\left[\begin{array}{l}
1 \\
0
\end{array}\right], \quad \vartheta_{3}=\vartheta\left[\begin{array}{l}
0 \\
0
\end{array}\right], \quad \vartheta_{4}=\vartheta\left[\begin{array}{l}
0 \\
1
\end{array}\right] .
$$

- The Appell-Lerch sum is defined as follows $[41]^{37}$ :

$$
\mu(u, \tau)=-\frac{e^{i \pi u}}{\vartheta_{1}(y, q)} \sum_{m \in \mathbb{Z}}(-1)^{m} \frac{e^{\pi i m(m+1) \tau+2 \pi i m u}}{1-e^{2 \pi i m \tau+2 \pi i u}},
$$

where

$$
y=\exp (2 \pi i u), \quad q=\exp (2 \pi i \tau) .
$$

\footnotetext{
36 These conventions are related to, for example, those adopted in Appendix A of [54] by $y=\exp (2 \pi i v), q=$ $\exp (2 \pi i \tau)$. We refer the reader to this reference for further properties of such functions.

37 The notation in this paper and that in Proposition 1.4 of [41] can be related as follows. Our notation is on the left-hand sides of the following equalities: $\mu(u, q)=\mu(u, u, q)$, and $\vartheta_{1}(u, \tau)=-\vartheta(u, \tau)$.
} 


\section{Appendix B. Data tables for super-Poincaré multiplicities}

This appendix contains data tables for multiplicities of super-Poincaré representations up to mass level $\alpha^{\prime} m^{2}=25$. We only display tables for the ancestor theories with 4, 8 and 16 supercharges, respectively, since these highest-dimensional theories organize the states in the most economic number of supermultiplets. Particular attention is paid to stable patterns, i.e. to the asymptotics of multiplicity generating functions for large spins and mass levels. Further tables of this kind which were displayed in an earlier version of the file can now be found in an AUXILIARY FILE 1.

Each of the following tables is devoted to family of supermultiplets whose quantum numbers differ in the first $S O(d-1)$ Dynkin label and match in the remaining $S O(d-1)$ and R-symmetry quantum numbers. Rows are associated with mass levels, and columns are associated with the value of the first $S O(d-1)$ Dynkin label to which we loosely refer to as the spin. Independently of spacetime dimensions and supercharges, the multiplicity generating functions $G_{\ldots}(q)$ tend to stabilize for large values of the spin and the mass level in the limit where both of them are uniformly increased. This leading Regge trajectory (corresponding to the $\tau_{1}^{\cdots}(q)$ contribution in (4.74), (5.42) and (6.34)) is exact when numbers occur repeatedly along diagonal lines in the tables, these entries are marked in red.

Moreover, once the asymptotic numbers in red are subtracted from the data outside the first stable region, further subleading trajectories emerge. The leftover after this subtraction tends to stabilize along lines where the mass level grows twice as fast as the spin. This can be understood as the second Regge trajectory (corresponding to the $\tau_{2}^{\cdots}(q)$ contribution in (4.74), (5.42) and (6.34)) with slope $\frac{1}{2}$ and subtractive sign. Its region of exact validity is highlighted in blue.

\section{B.1. 4 Supercharges in four dimensions}

Tables B.1, B.2 are based on the $\mathcal{N}_{4 d}=1$ partition function (4.4), organized in terms of multiplicity generating functions $G_{n, Q}(q)$, see (4.39).

\section{B.2. 8 supercharges in six dimensions}

Tables B.3, B.4, B.5 are based on the $\mathcal{N}_{6 d}=(1,0)$ partition function (5.3), organized in terms of multiplicity generating functions $G_{n_{1}, n_{2}, p}(q)$, see (5.23).

\section{B.3. 16 Supercharges in ten dimensions}

Tables B.6-B.9 are based on the $\mathcal{N}_{10 d}=1$ partition function (6.3), organized in terms of multiplicity generating functions $G_{n_{1}, n_{2}, n_{3}, n_{4}}(q)$, see (6.16).

\section{Appendix C. Deriving the asymptotic formulae for $\mathcal{N}_{4 d}=1$ multiplicity generating functions}

In this appendix, we derive the asymptotic results on multiplicity generating function $G_{n, Q}(q)$ in the limit $n \rightarrow \infty$ presented in Section 4.4.

In what follows, we will exploit the $n \rightarrow \infty$ behavior of objects $T_{p}(m, k):=\left(\begin{array}{c}m \\ k\end{array}\right)-\left(\begin{array}{c}m \\ k-p\end{array}\right)$, 


\begin{tabular}{|c|c|c|c|c|c|c|c|c|c|c|c|c|}
\hline$\alpha^{\prime} m^{2}$ & $\llbracket 1 ; 2 \rrbracket$ & $\llbracket 3 ; 2 \rrbracket$ & $\llbracket 5 ; 2 \rrbracket$ & $\llbracket 7 ; 2 \rrbracket$ & $\llbracket 9 ; 2 \rrbracket$ & $\llbracket 11 ; 2 \rrbracket$ & $\llbracket 13 ; 2 \rrbracket$ & $\llbracket 15 ; 2 \rrbracket$ & $\llbracket 17 ; 2 \rrbracket$ & $\llbracket 19 ; 2 \rrbracket$ & $\llbracket 21 ; 2 \rrbracket$ & $\llbracket 23 ; 2 \rrbracket$ \\
\hline 1 & 0 & & & & & & & & & & & \\
\hline 2 & 0 & & & & & & & & & & & \\
\hline 3 & 1 & 0 & & & & & & & & & & \\
\hline 4 & 2 & 2 & 0 & & & & & & & & & \\
\hline 5 & 6 & 6 & 2 & 0 & & & & & & & & \\
\hline 6 & 17 & 15 & 8 & 2 & 0 & & & & & & & \\
\hline 7 & 38 & 43 & 22 & 8 & 2 & 0 & & & & & & \\
\hline 8 & 89 & 101 & 62 & 24 & 8 & 2 & 0 & & & & & \\
\hline 9 & 195 & 233 & 152 & 71 & 24 & 8 & 2 & 0 & & & & \\
\hline 10 & 411 & 512 & 361 & 176 & 73 & 24 & 8 & 2 & 0 & & & \\
\hline 11 & 843 & 1089 & 803 & 430 & 185 & 73 & 24 & 8 & 2 & 0 & & \\
\hline 12 & 1694 & 2231 & 1734 & 978 & 456 & 187 & 73 & 24 & 8 & 2 & 0 & \\
\hline 13 & 3302 & 4483 & 3602 & 2146 & 1053 & 465 & 187 & 73 & 24 & 8 & 2 & 0 \\
\hline 14 & 6336 & 8758 & 7304 & 4525 & 2343 & 1079 & 467 & 187 & 73 & 24 & 8 & 2 \\
\hline 15 & 11919 & 16795 & 14402 & 9300 & 4997 & 2420 & 1088 & 467 & 187 & 73 & 24 & 8 \\
\hline 16 & 22053 & 31582 & 27835 & 18548 & 10383 & 5200 & 2446 & 1090 & 467 & 187 & 73 & 24 \\
\hline 17 & 40173 & 58428 & 52685 & 36227 & 20921 & 10878 & 5277 & 2455 & 1090 & 467 & 187 & 73 \\
\hline 18 & 72204 & 106359 & 98044 & 69217 & 41236 & 22068 & 11083 & 5303 & 2457 & 1090 & 467 & 187 \\
\hline 19 & 128014 & 191004 & 179419 & 129896 & 79473 & 43785 & 22569 & 11160 & 5312 & 2457 & 1090 & 467 \\
\hline 20 & 224337 & 338384 & 323661 & 239545 & 150345 & 84906 & 44955 & 22774 & 11186 & 5314 & 2457 & 1090 \\
\hline 21 & 388651 & 592391 & 575773 & 435174 & 279322 & 161591 & 87520 & 45458 & 22851 & 11195 & 5314 & 2457 \\
\hline 22 & 666314 & 1025226 & 1011672 & 779119 & 510970 & 301946 & 167204 & 88696 & 45663 & 22877 & 11197 & 5314 \\
\hline 23 & 1131024 & 1755809 & 1756589 & 1377070 & 920804 & 555389 & 313632 & 169841 & 89199 & 45740 & 22886 & 11197 \\
\hline 24 & 1902209 & 2976969 & 3017219 & 2404087 & 1637411 & 1006121 & 579053 & 319310 & 171019 & 89404 & 45766 & 22888 \\
\hline 25 & 3170935 & 5000934 & 5129359 & 4150179 & 2874993 & 1798156 & 1052851 & 590920 & 321953 & 171522 & 89481 & 45775 \\
\hline
\end{tabular}


Table B.2

\begin{tabular}{|c|c|c|c|c|c|c|c|c|c|c|c|}
\hline$\overline{\alpha^{\prime} m^{2}}$ & $\llbracket 0 ; 1 \rrbracket$ & $\llbracket 2 ; 1 \rrbracket$ & $\llbracket 4 ; 1 \rrbracket$ & $\llbracket 6 ; 1 \rrbracket$ & $\llbracket 8 ; 1 \rrbracket$ & $\llbracket 10 ; 1 \rrbracket$ & $\llbracket 12 ; 1 \rrbracket$ & $\llbracket 14 ; 1 \rrbracket$ & $\llbracket 16 ; 1 \rrbracket$ & $\llbracket 18 ; 1 \rrbracket$ & $\llbracket 20 ; 1 \rrbracket$ \\
\hline 1 & 1 & 0 & & & & & & & & & \\
\hline 2 & 0 & 2 & 0 & & & & & & & & \\
\hline 3 & 3 & 2 & 3 & 0 & & & & & & & \\
\hline 4 & 3 & 11 & 4 & 3 & 0 & & & & & & \\
\hline 5 & 15 & 20 & 18 & 5 & 3 & 0 & & & & & \\
\hline 6 & 21 & 58 & 39 & 21 & 5 & 3 & 0 & & & & \\
\hline 7 & 66 & 115 & 105 & 49 & 22 & 5 & 3 & 0 & & & \\
\hline 8 & 112 & 274 & 223 & 135 & 52 & 22 & 5 & 3 & 0 & & \\
\hline 9 & 267 & 543 & 521 & 296 & 146 & 53 & 22 & 5 & 3 & 0 & \\
\hline 10 & 487 & 1159 & 1066 & 698 & 330 & 149 & 53 & 22 & 5 & 3 & 0 \\
\hline 11 & 1027 & 2248 & 2258 & 1467 & 786 & 341 & 150 & 53 & 22 & 5 & 3 \\
\hline 12 & 1872 & 4483 & 4465 & 3133 & 1682 & 821 & 344 & 150 & 53 & 22 & 5 \\
\hline 13 & 3684 & 8456 & 8874 & 6300 & 3637 & 1774 & 832 & 345 & 150 & 53 & 22 \\
\hline 14 & 6654 & 16077 & 16929 & 12629 & 7413 & 3868 & 1809 & 835 & 345 & 150 & 53 \\
\hline 15 & 12430 & 29505 & 32174 & 24376 & 15014 & 7960 & 3961 & 1820 & 836 & 345 & 150 \\
\hline 16 & 22104 & 54085 & 59444 & 46663 & 29304 & 16246 & 8195 & 3996 & 1823 & 836 & 345 \\
\hline 17 & 39831 & 96778 & 109017 & 86997 & 56583 & 31974 & 16809 & 8288 & 4007 & 1824 & 836 \\
\hline 18 & 69495 & 172263 & 195931 & 160521 & 106459 & 62184 & 33250 & 17045 & 8323 & 4010 & 1824 \\
\hline 19 & 121751 & 301246 & 348996 & 290518 & 197927 & 117845 & 64978 & 33817 & 17138 & 8334 & 4011 \\
\hline 20 & 208588 & 523209 & 612069 & 520208 & 360936 & 220529 & 123748 & 66270 & 34053 & 17173 & 8337 \\
\hline 21 & 356951 & 896281 & 1063839 & 917434 & 650566 & 404759 & 232640 & 126586 & 66838 & 34146 & 17184 \\
\hline 22 & 601090 & 1524153 & 1825894 & 1601735 & 1154779 & 733851 & 428967 & 238668 & 127882 & 67074 & 34181 \\
\hline 23 & 1008432 & 2562971 & 3106955 & 2761714 & 2027692 & 1310137 & 781160 & 441385 & 241522 & 128450 & 67167 \\
\hline 24 & 1670909 & 4278549 & 5231334 & 4717314 & 3515675 & 2312784 & 1400641 & 806110 & 447457 & 242819 & 128686 \\
\hline 25 & 2755277 & 7075262 & 8737282 & 7973033 & 6035514 & 4030732 & 2482787 & 1449609 & 818653 & 450315 & 243387 \\
\hline
\end{tabular}




\begin{tabular}{|c|c|c|c|c|c|c|c|c|c|c|c|c|}
\hline$\alpha^{\prime} m^{2}$ & $\llbracket 0,2 ; 0 \rrbracket$ & $\llbracket 1,2 ; 0 \rrbracket$ & $\llbracket 2,2 ; 0 \rrbracket$ & $\llbracket 3,2 ; 0 \rrbracket$ & $\llbracket 4,2 ; 0 \rrbracket$ & $\llbracket 5,2 ; 0 \rrbracket$ & $\llbracket 6,2 ; 0 \rrbracket$ & $\llbracket 7,2 ; 0 \rrbracket$ & $\llbracket 8,2 ; 0 \rrbracket$ & $\llbracket 9,2 ; 0 \rrbracket$ & $\llbracket 10,2 ; 0 \rrbracket$ & $\llbracket 11,2 ; 0 \rrbracket$ \\
\hline 1 & 0 & & & & & & & & & & & \\
\hline 2 & 1 & 0 & & & & & & & & & & \\
\hline 3 & 1 & 1 & 0 & & & & & & & & & \\
\hline 4 & 4 & 2 & 1 & 0 & & & & & & & & \\
\hline 5 & 6 & 7 & 2 & 1 & 0 & & & & & & & \\
\hline 6 & 19 & 13 & 8 & 2 & 1 & 0 & & & & & & \\
\hline 7 & 34 & 38 & 16 & 8 & 2 & 1 & 0 & & & & & \\
\hline 8 & 81 & 79 & 48 & 17 & 8 & 2 & 1 & 0 & & & & \\
\hline 9 & 156 & 184 & 103 & 51 & 17 & 8 & 2 & 1 & 0 & & & \\
\hline 10 & 332 & 378 & 252 & 113 & 52 & 17 & 8 & 2 & 1 & 0 & & \\
\hline 11 & 636 & 813 & 530 & 279 & 116 & 52 & 17 & 8 & 2 & 1 & 0 & \\
\hline 12 & 1276 & 1623 & 1171 & 604 & 289 & 117 & 52 & 17 & 8 & 2 & 1 & 0 \\
\hline 13 & 2404 & 3290 & 2395 & 1350 & 631 & 292 & 117 & 52 & 17 & 8 & 2 & 1 \\
\hline 14 & 4614 & 6386 & 4962 & 2816 & 1427 & 641 & 293 & 117 & 52 & 17 & 8 & 2 \\
\hline 15 & 8537 & 12406 & 9823 & 5912 & 3001 & 1454 & 644 & 293 & 117 & 52 & 17 & 8 \\
\hline 16 & 15853 & 23445 & 19436 & 11896 & 6361 & 3078 & 1464 & 645 & 293 & 117 & 52 & 17 \\
\hline 17 & 28748 & 44075 & 37346 & 23836 & 12913 & 6549 & 3105 & 1467 & 645 & 293 & 117 & 52 \\
\hline 18 & 52034 & 81247 & 71315 & 46446 & 26104 & 13368 & 6626 & 3115 & 1468 & 645 & 293 & 117 \\
\hline 19 & 92579 & 148705 & 133388 & 89732 & 51295 & 27149 & 13556 & 6653 & 3118 & 1468 & 645 & 293 \\
\hline 20 & 163950 & 268145 & 247448 & 169908 & 99935 & 53631 & 27607 & 13633 & 6663 & 3119 & 1468 & 645 \\
\hline 21 & 286638 & 479693 & 451900 & 318623 & 190744 & 104983 & 54682 & 27795 & 13660 & 6666 & 3119 & 1468 \\
\hline 22 & 498178 & 848018 & 818105 & 588270 & 360520 & 201413 & 107347 & 55140 & 27872 & 13670 & 6667 & 3119 \\
\hline 23 & 856969 & 1487396 & 1462590 & 1075628 & 670688 & 382510 & 206529 & 108401 & 55328 & 27899 & 13673 & 6667 \\
\hline 24 & 1465054 & 2583018 & 2592572 & 1942043 & 1235427 & 715151 & 393379 & 208899 & 108859 & 55405 & 27909 & 13674 \\
\hline 25 & 2483037 & 4452127 & 4547623 & 3474093 & 2246578 & 1323605 & 737611 & 398523 & 209953 & 109047 & 55432 & 27912 \\
\hline
\end{tabular}


Table B.4

\begin{tabular}{|c|c|c|c|c|c|c|c|c|c|c|c|c|}
\hline$\alpha^{\prime} m^{2}$ & $\llbracket 0,0 ; 2 \rrbracket$ & $\llbracket 1,0 ; 2 \rrbracket$ & $\llbracket 2,0 ; 2 \rrbracket$ & $\llbracket 3,0 ; 2 \rrbracket$ & $\llbracket 4,0 ; 2 \rrbracket$ & $\llbracket 5,0 ; 2 \rrbracket$ & $\llbracket 6,0 ; 2 \rrbracket$ & $\llbracket 7,0 ; 2 \rrbracket$ & $\llbracket 8,0 ; 2 \rrbracket$ & $\llbracket 9,0 ; 2 \rrbracket$ & $\llbracket 10,0 ; 2 \rrbracket$ & $\llbracket 11,0 ; 2 \rrbracket$ \\
\hline 2 & 0 & & & & & & & & & & & \\
\hline 3 & 1 & 0 & & & & & & & & & & \\
\hline 4 & 0 & 2 & 0 & & & & & & & & & \\
\hline 5 & 3 & 3 & 3 & 0 & & & & & & & & \\
\hline 6 & 4 & 9 & 4 & 3 & 0 & & & & & & & \\
\hline 7 & 13 & 20 & 17 & 5 & 3 & 0 & & & & & & \\
\hline 8 & 20 & 50 & 34 & 19 & 5 & 3 & 0 & & & & & \\
\hline 9 & 53 & 101 & 93 & 43 & 20 & 5 & 3 & 0 & & & & \\
\hline 10 & 93 & 224 & 192 & 115 & 45 & 20 & 5 & 3 & 0 & & & \\
\hline 11 & 203 & 449 & 446 & 252 & 125 & 46 & 20 & 5 & 3 & 0 & & \\
\hline 12 & 369 & 924 & 903 & 589 & 275 & 127 & 46 & 20 & 5 & 3 & 0 & \\
\hline 13 & 743 & 1798 & 1920 & 1241 & 659 & 285 & 128 & 46 & 20 & 5 & 3 & 0 \\
\hline 14 & 1355 & 3523 & 3792 & 2664 & 1405 & 683 & 287 & 128 & 46 & 20 & 5 & 3 \\
\hline 15 & 2585 & 6673 & 7601 & 5410 & 3071 & 1476 & 693 & 288 & 128 & 46 & 20 & 5 \\
\hline 16 & 4662 & 12617 & 14601 & 10981 & 6311 & 3245 & 1500 & 695 & 288 & 128 & 46 & 20 \\
\hline 17 & 8585 & 23303 & 28083 & 21538 & 13007 & 6741 & 3317 & 1510 & 696 & 288 & 128 & 46 \\
\hline 18 & 15272 & 42800 & 52540 & 41953 & 25810 & 13982 & 6916 & 3341 & 1512 & 696 & 288 & 128 \\
\hline 19 & 27351 & 77315 & 97864 & 79808 & 50933 & 28012 & 14422 & 6988 & 3351 & 1513 & 696 & 288 \\
\hline 20 & 47902 & 138661 & 178789 & 150444 & 97964 & 55666 & 29010 & 14598 & 7012 & 3353 & 1513 & 696 \\
\hline 21 & 83950 & 245476 & 324415 & 278690 & 186802 & 107982 & 57944 & 29451 & 14670 & 7022 & 3354 & 1513 \\
\hline 22 & 144814 & 431357 & 580136 & 511315 & 349601 & 207363 & 112896 & 58952 & 29627 & 14694 & 7024 & 3354 \\
\hline 23 & 249137 & 750026 & 1029661 & 925300 & 648055 & 391117 & 217862 & 115197 & 59394 & 29699 & 14704 & 7025 \\
\hline 24 & 423589 & 1294613 & 1806340 & 1658994 & 1183895 & 730037 & 412771 & 222852 & 116206 & 59570 & 29723 & 14706 \\
\hline 25 & 717200 & 2214733 & 3145140 & 2940833 & 2142556 & 1343353 & 774118 & 423453 & 225163 & 116648 & 59642 & 29733 \\
\hline
\end{tabular}




\begin{tabular}{|c|c|c|c|c|c|c|c|c|c|c|c|c|}
\hline$\alpha^{\prime} m^{2}$ & $\llbracket 0,1 ; 1 \rrbracket$ & $\llbracket 1,1 ; 1 \rrbracket$ & $\llbracket 2,1 ; 1 \rrbracket$ & $\llbracket 3,1 ; 1 \rrbracket$ & $\llbracket 4,1 ; 1 \rrbracket$ & $\llbracket 5,1 ; 1 \rrbracket$ & $\llbracket 6,1 ; 1 \rrbracket$ & $\llbracket 7,1 ; 1 \rrbracket$ & $\llbracket 8,1 ; 1 \rrbracket$ & $\llbracket 9,1 ; 1 \rrbracket$ & $\llbracket 10,1 ; 1 \rrbracket$ & $\llbracket 11,1 ; 1 \rrbracket$ \\
\hline 1 & 0 & & & & & & & & & & & \\
\hline 2 & 1 & 0 & & & & & & & & & & \\
\hline 3 & 1 & 2 & 0 & & & & & & & & & \\
\hline 4 & 4 & 3 & 2 & 0 & & & & & & & & \\
\hline 5 & 8 & 9 & 4 & 2 & 0 & & & & & & & \\
\hline 6 & 18 & 23 & 12 & 4 & 2 & 0 & & & & & & \\
\hline 7 & 39 & 51 & 31 & 13 & 4 & 2 & 0 & & & & & \\
\hline 8 & 82 & 114 & 76 & 34 & 13 & 4 & 2 & 0 & & & & \\
\hline 9 & 165 & 249 & 174 & 85 & 35 & 13 & 4 & 2 & 0 & & & \\
\hline 10 & 333 & 519 & 391 & 203 & 88 & 35 & 13 & 4 & 2 & 0 & & \\
\hline 11 & 652 & 1064 & 843 & 465 & 212 & 89 & 35 & 13 & 4 & 2 & 0 & \\
\hline 12 & 1260 & 2137 & 1776 & 1024 & 495 & 215 & 89 & 35 & 13 & 4 & 2 & 0 \\
\hline 13 & 2396 & 4202 & 3645 & 2203 & 1102 & 504 & 216 & 89 & 35 & 13 & 4 & 2 \\
\hline 14 & 4499 & 8128 & 7330 & 4609 & 2399 & 1132 & 507 & 216 & 89 & 35 & 13 & 4 \\
\hline 15 & 8321 & 15488 & 14450 & 9428 & 5080 & 2478 & 1141 & 508 & 216 & 89 & 35 & 13 \\
\hline 16 & 15236 & 29063 & 28022 & 18898 & 10511 & 5280 & 2508 & 1144 & 508 & 216 & 89 & 35 \\
\hline 17 & 27556 & 53844 & 53451 & 37201 & 21297 & 10997 & 5359 & 2517 & 1145 & 508 & 216 & 89 \\
\hline 18 & 49336 & 98540 & 100527 & 71985 & 42376 & 22425 & 11198 & 5389 & 2520 & 1145 & 508 & 216 \\
\hline 19 & 87449 & 178260 & 186521 & 137212 & 82828 & 44899 & 22915 & 11277 & 5398 & 2521 & 1145 & 508 \\
\hline 20 & 153595 & 319063 & 341843 & 257835 & 159430 & 88321 & 46042 & 23116 & 11307 & 5401 & 2521 & 1145 \\
\hline 21 & 267352 & 565412 & 619252 & 478197 & 302417 & 171054 & 90889 & 46533 & 23195 & 11316 & 5402 & 2521 \\
\hline 22 & 461595 & 992485 & 1109824 & 876142 & 565992 & 326453 & 176672 & 92036 & 46734 & 23225 & 11319 & 5402 \\
\hline 23 & 790578 & 1726764 & 1968850 & 1587104 & 1046065 & 614658 & 338400 & 179255 & 92527 & 46813 & 23234 & 11320 \\
\hline 24 & 1343972 & 2979088 & 3459778 & 2844391 & 1910959 & 1142740 & 639492 & 344063 & 180403 & 92728 & 46843 & 23237 \\
\hline 25 & 2268336 & 5098709 & 6025145 & 5046950 & 3452679 & 2099666 & 1193279 & 651564 & 346650 & 180894 & 92807 & 46852 \\
\hline
\end{tabular}


Table B.6

\begin{tabular}{|c|c|c|c|c|c|c|c|c|c|c|c|c|c|c|c|}
\hline$\alpha^{\prime} n$ & , $1,0,0 \rrbracket$ & $1,1,0,0]$ & $\llbracket 2,1,0,0$ & {$[3,1,0,0]$} & {$[4,1,0,0$} & $\llbracket 5,1,0,0$ & $\llbracket 6,1,0,0$ & $7,1,0,0$ & $, 1,0,0]$ & $, 1,0,0]$ & $0,1,0$, & , 1, 0, & $, 1,0$, & $, 1,0$, & $, 1,0,0]$ \\
\hline 3 & 0 & & & & & & & & & & & & & & \\
\hline 4 & 1 & 0 & & & & & & & & & & & & & \\
\hline 5 & 1 & 1 & 0 & & & & & & & & & & & & \\
\hline 6 & 1 & 2 & 1 & 0 & & & & & & & & & & & \\
\hline 7 & 2 & 2 & 2 & 1 & 0 & & & & & & & & & & \\
\hline 8 & 5 & 5 & 3 & 2 & 1 & 0 & & & & & & & & & \\
\hline 9 & 7 & 9 & 6 & 3 & 2 & 1 & 0 & & & & & & & & \\
\hline 10 & 13 & 17 & 12 & 7 & 3 & 2 & 1 & 0 & & & & & & & \\
\hline 11 & 21 & 29 & 23 & 13 & 7 & 3 & 2 & 1 & 0 & & & & & & \\
\hline 12 & 37 & 54 & 42 & 26 & 14 & 7 & 3 & 2 & 1 & 0 & & & & & \\
\hline 13 & 60 & 90 & 77 & 48 & 27 & 14 & 7 & 3 & 2 & 1 & 0 & & & & \\
\hline 14 & 101 & 159 & 137 & 92 & 51 & 28 & 14 & 7 & 3 & 2 & 1 & 0 & & & \\
\hline 15 & 165 & 268 & 243 & 163 & 98 & 52 & 28 & 14 & 7 & 3 & 2 & 1 & 0 & & \\
\hline 16 & 274 & 457 & 422 & 298 & 178 & 101 & 53 & 28 & 14 & 7 & 3 & 2 & 1 & 0 & \\
\hline 17 & 441 & 760 & 732 & 522 & 326 & 184 & 102 & 53 & 28 & 14 & 7 & 3 & 2 & 1 & 0 \\
\hline 18 & 717 & 1276 & 1248 & 924 & 580 & 341 & 187 & 103 & 53 & 28 & 14 & 7 & 3 & 2 & 1 \\
\hline 19 & 1149 & 2088 & 2121 & 1592 & 1032 & 608 & 347 & 188 & 103 & 53 & 28 & 14 & 7 & 3 & 2 \\
\hline 20 & 1847 & 3443 & 3551 & 2750 & 1801 & 1092 & 623 & 350 & 189 & 103 & 53 & 28 & 14 & 7 & 3 \\
\hline 21 & 2928 & 5585 & 5929 & 4656 & 3134 & 1912 & 1120 & 629 & 351 & 189 & 103 & 53 & 28 & 14 & 7 \\
\hline 22 & 4647 & 9060 & 9790 & 7886 & 5361 & 3351 & 1972 & 1135 & 632 & 352 & 189 & 103 & 53 & 28 & 14 \\
\hline 23 & 7310 & 14538 & 16095 & 13160 & 9148 & 5762 & 3464 & 2000 & 1141 & 633 & 352 & 189 & 103 & 53 & 28 \\
\hline 24 & 11482 & 23301 & 26221 & 21906 & 15414 & 9894 & 5982 & 3524 & 2015 & 1144 & 634 & 352 & 189 & 103 & 53 \\
\hline 25 & 17908 & 36995 & 42535 & 36063 & 25846 & 16754 & 10303 & 6095 & 3552 & 2021 & 1145 & 634 & 352 & 189 & 103 \\
\hline
\end{tabular}


Table B.7

\begin{tabular}{|c|c|c|c|c|c|c|c|c|c|c|c|c|c|c|c|}
\hline$\alpha^{\prime} n$ & $0,0,1,0$ & $1,0,1,0$ & $2,0,1,0$ & $3,0,1,0$ & $4,0,1,0$ & $5,0,1,0 \rrbracket$ & $6,0,1,0 \rrbracket$ & $, 0,1,0]$ & $, 0,1,0 \rrbracket$ & $, 0,1,0]$ & $0,0,1$, & $, 0,1$, & 0,1 , & 0,1 , & $0,1,0]$ \\
\hline 4 & 0 & & & & & & & & & & & & & & \\
\hline 5 & 1 & 0 & & & & & & & & & & & & & \\
\hline 6 & 0 & 1 & 0 & & & & & & & & & & & & \\
\hline 7 & 3 & 1 & 1 & 0 & & & & & & & & & & & \\
\hline 8 & 2 & 4 & 1 & 1 & 0 & & & & & & & & & & \\
\hline 9 & 7 & 6 & 5 & 1 & 1 & 0 & & & & & & & & & \\
\hline 10 & 10 & 15 & 7 & 5 & 1 & 1 & 0 & & & & & & & & \\
\hline 11 & 22 & 24 & 20 & 8 & 5 & 1 & 1 & 0 & & & & & & & \\
\hline 12 & 30 & 51 & 33 & 21 & 8 & 5 & 1 & 1 & 0 & & & & & & \\
\hline 13 & 64 & 85 & 73 & 38 & 22 & 8 & 5 & 1 & 1 & 0 & & & & & \\
\hline 14 & 97 & 164 & 125 & 83 & 39 & 22 & 8 & 5 & 1 & 1 & 0 & & & & \\
\hline 15 & 179 & 276 & 249 & 148 & 88 & 40 & 22 & 8 & 5 & 1 & 1 & 0 & & & \\
\hline 16 & 282 & 502 & 431 & 297 & 158 & 89 & 40 & 22 & 8 & 5 & 1 & 1 & 0 & & \\
\hline 17 & 496 & 842 & 803 & 529 & 321 & 163 & 90 & 40 & 22 & 8 & 5 & 1 & 1 & 0 & \\
\hline 18 & 784 & 1473 & 1379 & 993 & 578 & 331 & 164 & 90 & 40 & 22 & 8 & 5 & 1 & 1 & 0 \\
\hline 19 & 1335 & 2449 & 2462 & 1748 & 1099 & 602 & 336 & 165 & 90 & 40 & 22 & 8 & 5 & 1 & 1 \\
\hline 20 & 2117 & 4164 & 4181 & 3153 & 1951 & 1149 & 612 & 337 & 165 & 90 & 40 & 22 & 8 & 5 & 1 \\
\hline 21 & 3497 & 6853 & 7238 & 5454 & 3559 & 2058 & 1173 & 617 & 338 & 165 & 90 & 40 & 22 & 8 & 5 \\
\hline 22 & 5546 & 11401 & 12131 & 9549 & 6218 & 3770 & 2108 & 1183 & 618 & 338 & 165 & 90 & 40 & 22 & 8 \\
\hline 23 & 8981 & 18557 & 20509 & 16261 & 10990 & 6637 & 3878 & 2132 & 1188 & 619 & 338 & 165 & 90 & 40 & 22 \\
\hline 24 & 14141 & 30342 & 33931 & 27794 & 18890 & 11791 & 6849 & 3928 & 2142 & 1189 & 619 & 338 & 165 & 90 & 40 \\
\hline 25 & 22570 & 48846 & 56288 & 46628 & 32585 & 20406 & 12218 & 6957 & 3952 & 2147 & 1190 & 619 & 338 & 165 & 90 \\
\hline
\end{tabular}


Table B. 8

\begin{tabular}{|c|c|c|c|c|c|c|c|c|c|c|c|c|c|c|c|}
\hline$\alpha^{\prime} m$ & $0,0,2]$ & $1,0,0,2 \rrbracket$ & $2,0,0,2$ & {$[3,0,0,2$} & $4,0,0,2]$ & {$[5,0,0,2]$} & $6,0,0,2$ & $0,0,2$ & $0,0,2$ & $, 0,0,2 \rrbracket$ & $0,0,0$ & $, 0,0$, & 0,0 & 0,0, & $0,0,2 \rrbracket$ \\
\hline 5 & 0 & & & & & & & & & & & & & & \\
\hline 6 & 1 & 0 & & & & & & & & & & & & & \\
\hline 7 & 1 & 1 & 0 & & & & & & & & & & & & \\
\hline 8 & 3 & 2 & 1 & 0 & & & & & & & & & & & \\
\hline 9 & 4 & 6 & 2 & 1 & 0 & & & & & & & & & & \\
\hline 10 & 10 & 9 & 7 & 2 & 1 & 0 & & & & & & & & & \\
\hline 11 & 16 & 22 & 12 & 7 & 2 & 1 & 0 & & & & & & & & \\
\hline 12 & 32 & 40 & 29 & 13 & 7 & 2 & 1 & 0 & & & & & & & \\
\hline 13 & 52 & 80 & 55 & 32 & 13 & 7 & 2 & 1 & 0 & & & & & & \\
\hline 14 & 98 & 141 & 115 & 62 & 33 & 13 & 7 & 2 & 1 & 0 & & & & & \\
\hline 15 & 160 & 267 & 211 & 132 & 65 & 33 & 13 & 7 & 2 & 1 & 0 & & & & \\
\hline 16 & 286 & 463 & 409 & 249 & 139 & 66 & 33 & 13 & 7 & 2 & 1 & 0 & & & \\
\hline 17 & 469 & 835 & 733 & 491 & 266 & 142 & 66 & 33 & 13 & 7 & 2 & 1 & 0 & & \\
\hline 18 & 805 & 1431 & 1351 & 900 & 531 & 273 & 143 & 66 & 33 & 13 & 7 & 2 & 1 & 0 & \\
\hline 19 & 1314 & 2489 & 2375 & 1685 & 985 & 548 & 276 & 143 & 66 & 33 & 13 & 7 & 2 & 1 & 0 \\
\hline 20 & 2199 & 4199 & 4218 & 3018 & 1864 & 1025 & 555 & 277 & 143 & 66 & 33 & 13 & 7 & 2 & 1 \\
\hline 21 & 3558 & 7131 & 7270 & 5438 & 3378 & 1951 & 1042 & 558 & 277 & 143 & 66 & 33 & 13 & 7 & 2 \\
\hline 22 & 5837 & 11842 & 12571 & 9530 & 6148 & 3560 & 1991 & 1049 & 559 & 277 & 143 & 66 & 33 & 13 & 7 \\
\hline 23 & 9361 & 19709 & 21279 & 16701 & 10888 & 6520 & 3647 & 2008 & 1052 & 559 & 277 & 143 & 66 & 33 & 13 \\
\hline 24 & 15106 & 32300 & 35990 & 28688 & 19266 & 11624 & 6704 & 3687 & 2015 & 1053 & 559 & 277 & 143 & 66 & 33 \\
\hline 25 & 23999 & 52855 & 59966 & 49138 & 33418 & 20692 & 11999 & 6791 & 3704 & 2018 & 1053 & 559 & 277 & 143 & 66 \\
\hline
\end{tabular}


Table B.9

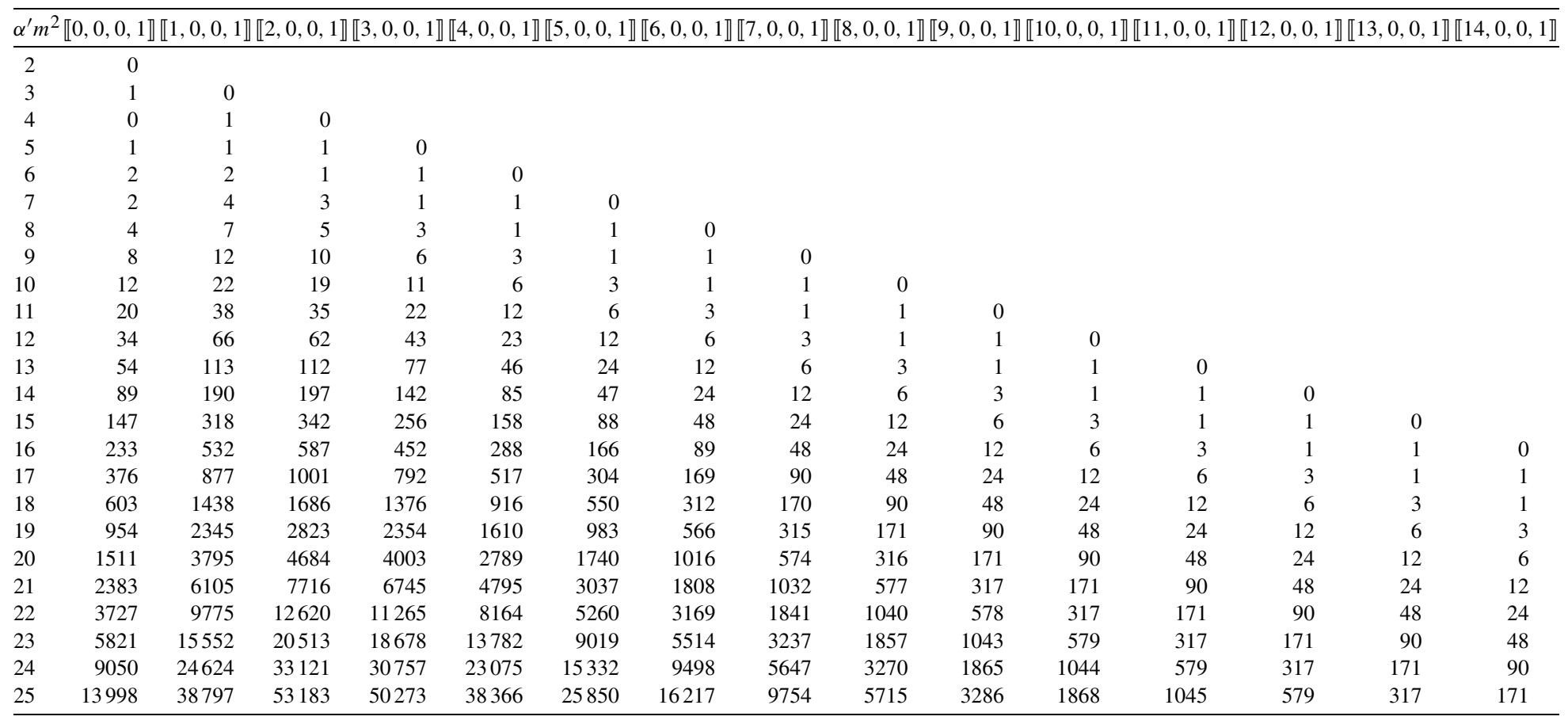




$$
\begin{aligned}
& T_{2 n+2}(2 m+1, m+n+1-k) \sim\left(\begin{array}{c}
2 m+1 \\
m+n+1-k
\end{array}\right), \\
& T_{2 n+2}(2 m, m+n-k) \sim\left(\begin{array}{c}
2 m \\
m+n-k
\end{array}\right)
\end{aligned}
$$

assuming that $m, k \geqslant 0$.

\section{C.1. Warm-up: Multiplicities of $\llbracket 2 n+1,0 \rrbracket$ and $\llbracket 2 n, 1 \rrbracket$ as $n \rightarrow \infty$}

In order to get familiar with the asymptotic methods in the $\mathcal{N}_{4 d}=1$ context, we shall first of all discuss the large spin regime of supermultiplets with $U(1)_{R}$ neutral Clifford vacuum.

The multiplicity generating function for the representation $\llbracket 2 n+1,0 \rrbracket$ can be written as

$$
G_{2 n+1,0}(q)=\sum_{k=0}^{\infty} \sum_{m=0}^{\infty} \sum_{p=0}^{\infty} \mathfrak{M}_{\llbracket 2 n+1,0 \rrbracket}(m,-p-1, k ; q)+\sum_{k=0}^{\infty} \sum_{p=0}^{\infty} \mathfrak{M}_{\llbracket 2 n+1,0 \rrbracket}(p, p, k ; q),
$$

where the function $\mathfrak{M}_{\llbracket 2 n+1,2 Q \rrbracket}$ and $\mathfrak{M}_{\llbracket 2 n+1,2 Q \rrbracket}$ are defined in (4.59) and (4.60) and, as $n \rightarrow \infty$,

$$
\begin{aligned}
\mathfrak{M}_{\llbracket 2 n+1,0 \rrbracket}(m, p, k ; q) \sim & (-1)^{-m-p}\left[F_{k, p}^{\mathrm{NS}}(q)\left(\begin{array}{c}
m-p \\
2 m+1
\end{array}\right)\left(\begin{array}{c}
2 m+1 \\
m+n+1-k
\end{array}\right)\right. \\
& \left.+F_{k, p}^{\mathrm{R}}(q)\left(\begin{array}{c}
m-p \\
2 m
\end{array}\right)\left(\begin{array}{c}
2 m \\
m+n-k
\end{array}\right)\right] .
\end{aligned}
$$

Note that the binomial coefficient $\left(\begin{array}{l}\alpha \\ \beta\end{array}\right)$ increases as $\beta$ increases from 0 to $\lfloor\alpha / 2\rfloor$ and then decreases as $\beta$ increases from $\lfloor\alpha / 2\rfloor+1$ to $\alpha$.

Observe that $\mathfrak{M}_{\llbracket 2 n+1,0 \rrbracket}(m,-p-1, k ; q)$ is sharply peaked near $(m, p, k)=(0,0, n)$ for $n$ large. Therefore, the dominant contribution to the first set of summations in (C.2) comes from

$$
\begin{aligned}
& \sum_{m=0}^{\infty} \sum_{p=0}^{\infty} \sum_{k=0}^{\infty} \mathfrak{M}_{\llbracket 2 n+1,0 \rrbracket}(m,-p-1, k ; q) \\
& \sim \sum_{m=0}^{\left\lceil\epsilon_{1}\right\rceil} \sum_{p=0}^{\left\lceil\epsilon_{2}\right\rceil} \sum_{k=\left\lfloor n\left(1-\epsilon_{3}\right)\right\rfloor}^{\left\lceil n\left(1+\epsilon_{3}\right)\right\rceil} \mathfrak{M}_{\llbracket 2 n+1,0 \rrbracket}(m,-p-1, k ; q) \text { any } \epsilon_{1}, \epsilon_{2}, \epsilon_{3}>0, n \rightarrow \infty \\
& \quad \sim \sum_{m=0}^{\infty} \sum_{p=0}^{\infty} \sum_{\delta=-\infty}^{\infty} \mathfrak{M}_{\llbracket 2 n+1,0 \rrbracket}(m,-p-1, n+\delta ; q), \quad n \rightarrow \infty .
\end{aligned}
$$

In the limit of large $k$, we can use asymptotic formulae (4.23) and (4.28) for $F_{k, p}^{\mathrm{NS}}(q)$ and $F_{k, p}^{\mathrm{R}}(q)$. The summation over $\delta$ from $-\infty$ to $\infty$ can be readily computed using the fact that

$$
\begin{aligned}
& \sum_{\delta=-\infty}^{\infty} q^{\delta}\left(\begin{array}{c}
2 m \\
m-\delta
\end{array}\right)=\sum_{\delta=-m}^{m} q^{\delta}\left(\begin{array}{c}
2 m \\
m-\delta
\end{array}\right)=q^{-m}(1+q)^{2 m} \\
& \sum_{\delta=-\infty}^{\infty} q^{\delta}\left(\begin{array}{c}
2 m+1 \\
m-\delta+1
\end{array}\right)=\sum_{\delta=-(m+1)}^{m+1} q^{\delta}\left(\begin{array}{c}
2 m+1 \\
m-\delta+1
\end{array}\right)=q^{-m}(1+q)^{2 m+1}
\end{aligned}
$$

Next, the summation over $m$ from 0 to $\infty$ can be computed using the following identities: 


$$
\begin{aligned}
& \sum_{m=0}^{\infty}(-q)^{-m}(1+q)^{2 m}\left(\begin{array}{c}
1+m+p \\
2 m
\end{array}\right)=(-q)^{-p-1} \frac{1-q^{2 p+3}}{1-q} \\
& \sum_{m=0}^{\infty}(-q)^{-m}(1+q)^{2 m+1}\left(\begin{array}{c}
1+m+p \\
1+2 m
\end{array}\right)=(-q)^{-p} \frac{1-q^{2 p+2}}{1-q}
\end{aligned}
$$

Thus, from (C.4), we find that

$$
\begin{aligned}
& \sum_{m=0}^{\infty} \sum_{p=0}^{\infty} \sum_{k=0}^{\infty} \mathfrak{M}_{\llbracket 2 n+1,0 \rrbracket}(m,-p-1, k ; q) \\
& \quad=\frac{(1-q)^{2} q^{n-\frac{1}{2}}}{2(q, q)_{\infty}^{6}}\left\{u_{1}(\sqrt{q}) \vartheta_{2}(1, q)^{2}-\left[u_{2}(\sqrt{q}) \vartheta_{3}(1, q)^{2}-u_{2}(-\sqrt{q}) \vartheta_{4}(1, q)^{2}\right]\right\},
\end{aligned}
$$

where the functions $u_{1}(q)$ and $u_{2}(q)$ are defined as follows:

$$
\begin{aligned}
& u_{1}(q)=\sum_{p=0}^{\infty} q^{2\left(p+\frac{3}{2}\right)^{2}} \frac{1-q^{4 p+6}}{\left(1+q^{2 p+2}\right)\left(1+q^{2 p+4}\right)} \\
& u_{2}(q)=\sum_{p=0}^{\infty} q^{2(p+1)^{2}} \frac{1-q^{4 p+4}}{\left(1+q^{2 p+1}\right)\left(1+q^{2 p+3}\right)}
\end{aligned}
$$

It remains unclear whether $u_{1}(q)$ and $u_{2}(q)$ can be written in terms of known functions (if this is useful at all). In practice, it is easy to compute the power series $u_{1}(q)$ and $u_{2}(q)$ up to a high order in $q$. Moreover, their asymptotic formulae can be easily derived in the limit $q \rightarrow 0$. We shall come back to this point later.

Let us now examine the second set of summations in (C.2). The function $\mathfrak{M}_{\llbracket 2 n+1,0 \rrbracket}(p, p, k ; q)$ is sharply peaked near $(p, k)=(0, n)$ for large $n$. Thus,

$$
\begin{aligned}
\sum_{k=0}^{\infty} \sum_{p=0}^{\infty} \mathfrak{M}_{\llbracket 2 n+1,0 \rrbracket}(p, p, k ; q) & \sim \mathfrak{M}_{\llbracket 2 n+1,0 \rrbracket}(0,0, n ; q), \quad n \rightarrow \infty \\
& =\frac{1}{4(q ; q)_{\infty}^{6}} \frac{(1-q)^{3}}{1+q} q^{n-\frac{1}{4}} \vartheta_{2}(1, q)^{2}
\end{aligned}
$$

From (C.2), we simply add (C.4) and (C.9) together and obtain the expression (4.72) for $Q_{2 n+1,0}$, in agreement with the stable pattern in Table 3 .

From recurrence relation (4.35) for $G_{n, Q}$, the asymptotic behavior of multiplicity generating functions $U(1)_{R}$ charge $Q=1$ is given by

$$
G_{2 n, 1}(q)=\frac{1}{2}\left[F_{n, 0}^{\mathrm{NS}}(q)-G_{2 n-1,0}(q)-G_{2 n+1,0}(q)\right] .
$$

Using the asymptotics $G_{2 n-1, Q} \sim q^{-1} G_{2 n+1, Q}$ as well as (4.72) for $G_{2 n+1, Q}$ and (4.23) for $F_{n, 0}^{\mathrm{NS}}$, we arrive at (4.73). This also agrees with the stable pattern tabulated in Appendix B.1. 
C.2. Multiplicities of $\llbracket 2 n+1,2 Q \rrbracket$ and $\llbracket 2 n, 2 Q+1 \rrbracket$ as $n \rightarrow \infty, Q=\mathcal{O}(1)$

This subsection generalizes the asymptotic results from the $Q=0$ (or $Q=1$ ) sector to generic $U(1)_{R}$ charges. The multiplicity generating function for $\llbracket 2 n+1,2 Q \rrbracket$ can be written as

$$
\begin{aligned}
G_{2 n+1,2 Q}(q)= & \sum_{k=0}^{\infty} \sum_{m=0}^{\infty}\left[\sum _ { p = 0 } ^ { \infty } \left\{\mathfrak{M}_{\llbracket 2 n+1,2 Q \rrbracket}(m,-p-1, k ; q)\right.\right. \\
& \left.+\mathfrak{M}_{\llbracket 2 n+1,2 Q \rrbracket}(m+p, p, k ; q)\right\} \\
& \left.+\sum_{p=0}^{Q-1} \mathfrak{M}_{\llbracket 2 n+1,2 Q \rrbracket}(m, m+p+1, k ; q)\right]
\end{aligned}
$$

where the $\mathfrak{M}_{\llbracket 2 n+1,2 Q \rrbracket}$ function follows the following $n \rightarrow \infty$ behavior:

$$
\begin{aligned}
\mathfrak{M}_{\llbracket 2 n+1,2 Q \rrbracket}(m, p, k ; q)= & (-1)^{Q-m-p}\left[F_{k, p}^{\mathrm{NS}}(q)\left(\begin{array}{c}
Q+m-p \\
2 m+1
\end{array}\right)\left(\begin{array}{c}
2 m+1 \\
m+n+1-k
\end{array}\right)\right. \\
& \left.+F_{k, p}^{\mathrm{R}}(q)\left(\begin{array}{c}
Q+m-p \\
2 m
\end{array}\right)\left(\begin{array}{c}
2 m \\
m+n-k
\end{array}\right)\right] .
\end{aligned}
$$

The dominant contribution to $G_{2 n+1,2 Q}(q)$ comes from

$$
\begin{aligned}
G_{2 n+1,2 Q}(q) & \sim \sum_{m=0}^{\infty} \sum_{p=0}^{\left\lceil\epsilon_{2}\right\rceil} \sum_{k=\left\lfloor n\left(1-\epsilon_{1}\right)\right\rfloor}^{\left\lceil n\left(1+\epsilon_{1}\right)\right\rceil}\left[\mathfrak{M}_{\llbracket 2 n+1,2 Q \rrbracket}(m,-p-1, k ; q)+\mathfrak{M}_{\llbracket 2 n+1,2 Q \rrbracket}(m+p, p, k ; q)\right] \\
& +\sum_{m=0}^{\infty} \sum_{p=0}^{Q-1} \sum_{k=\left\lfloor n\left(1-\epsilon_{1}\right)\right\rfloor}^{\left\lceil n\left(1+\epsilon_{1}\right)\right\rceil} \mathfrak{M}_{\llbracket 2 n+1,2 Q \rrbracket}(m, m+p+1, k ; q), \quad \epsilon_{1}, \epsilon_{2}>0, n \rightarrow \infty \\
\sim & \sum_{m=0}^{\infty} \sum_{p=0}^{\infty} \sum_{\delta=-\infty}^{\infty}\left[\mathfrak{M}_{\llbracket 2 n+1,2 Q \rrbracket}(m,-p-1, n+\delta ; q)\right. \\
& \left.+\mathfrak{M}_{\llbracket 2 n+1,2 Q \rrbracket}(m+p, p, n+\delta ; q)\right] \\
& +\sum_{m=0}^{\infty} \sum_{p=0}^{Q-1} \sum_{\delta=-\infty}^{\infty} \mathfrak{M}_{\llbracket 2 n+1,2 Q \rrbracket}(m, m+p+1, n+\delta ; q), \quad n \rightarrow \infty .
\end{aligned}
$$

The first set of summations can be evaluated as follows:

$$
\begin{aligned}
\sum_{m=0}^{\infty} \sum_{p=0}^{\infty} \sum_{\delta=-\infty}^{\infty} \mathfrak{M}_{\llbracket 2 n+1,2 Q \rrbracket}(m,-p-1, n+\delta ; q) \\
=\frac{(1-q)^{2} q^{n-Q-\frac{1}{2}}}{2(q ; q)_{\infty}^{6}}\left\{u_{1}(\sqrt{q}, Q) \vartheta_{2}(1, q)^{2}\right. \\
\left.\quad-\left[u_{2}(\sqrt{q}, Q) \vartheta_{3}(1, q)^{2}-u_{2}(-\sqrt{q}, Q) \vartheta_{4}(1, q)^{2}\right]\right\}
\end{aligned}
$$

where 


$$
\begin{aligned}
& u_{1}(q, Q)=\sum_{p=0}^{\infty} q^{2\left(p+\frac{3}{2}\right)^{2}} \frac{1-q^{4 p+4 Q+6}}{\left(1+q^{2 p+2}\right)\left(1+q^{2 p+4}\right)}, \\
& u_{2}(q, Q)=\sum_{p=0}^{\infty} q^{2(p+1)^{2}} \frac{1-q^{4 p+4 Q+4}}{\left(1+q^{2 p+1}\right)\left(1+q^{2 p+3}\right)} .
\end{aligned}
$$

The next set of summations in (4.62) can be evaluated as follows:

$$
\begin{aligned}
\sum_{m=0}^{\infty} \sum_{p=0}^{\infty} \sum_{\delta=-\infty}^{\infty} \mathfrak{M}_{\llbracket 2 n+1,2 Q \rrbracket}(m+p, p, n+\delta ; q) \\
=\frac{(-1)^{Q}(1-q)^{3} q^{n-\frac{3}{2}}}{2(q ; q)_{\infty}^{6}}\left\{v_{1}(\sqrt{q}, Q) \vartheta_{2}(1, q)^{2}\right. \\
\left.\quad+\left[v_{2}(\sqrt{q}, Q) \vartheta_{3}(1, q)^{2}-v_{2}(-\sqrt{q}, Q) \vartheta_{4}(1, q)^{2}\right]\right\},
\end{aligned}
$$

where $^{38}$

$$
\begin{aligned}
& v_{1}(q, Q)=\sum_{p=0}^{\lfloor Q / 2\rfloor} \frac{q^{2\left(p-\frac{1}{2}\right)^{2}}\left(1+q^{2}\right)^{2 p}}{\left(1+q^{2 p-2}\right)\left(1+q^{2 p}\right)}\left(\begin{array}{c}
Q \\
2 p
\end{array}\right){ }_{3} F_{2}\left[\begin{array}{c}
1, Q+1,2 p-Q \\
p+1 / 2, p+1
\end{array} ; \frac{(1+q)^{2}}{4 q}\right], \\
& v_{2}(q, Q)=\sum_{p=0}^{\lfloor Q / 2\rfloor} \frac{(1+q) q^{2 p^{2}}\left(1+q^{2}\right)^{2 p}}{\left(1+q^{2 p-1}\right)\left(1+q^{2 p+1}\right)}\left(\begin{array}{c}
Q \\
2 p+1
\end{array}\right){ }_{3} F_{2}\left[\begin{array}{c}
1, Q+1,2 p+1-Q \\
p+1, p+3 / 2
\end{array} ; \frac{(1+q)^{2}}{4 q}\right] .
\end{aligned}
$$

The last set of summations in (4.62) can be evaluated as follows:

$$
\begin{aligned}
\sum_{m=0}^{\infty} & \sum_{p=0}^{Q-1} \sum_{\delta=-\infty}^{\infty} \mathfrak{M}_{\llbracket 2 n+1,2 Q \rrbracket}(m, m+p+1, n+\delta ; q) \\
= & \frac{(-1)^{Q}(1-q)^{3} q^{n-\frac{7}{4}}}{2(q ; q)_{\infty}^{6}}\left\{w_{1}(\sqrt{q}, Q) \vartheta_{2}(1, q)^{2}\right. \\
& \left.+q^{\frac{9}{4}}\left[w_{2}(\sqrt{q}, Q) \vartheta_{3}(1, q)^{2}-w_{2}(-\sqrt{q}, Q) \vartheta_{4}(1, q)^{2}\right]\right\}
\end{aligned}
$$

where

$$
w_{1}(q, Q)=\sum_{m=0}^{\infty} \sum_{p=0}^{Q-1} \frac{(-1)^{p+1} q^{1+2(1+m+p)^{2}-2 m}\left(1+q^{2}\right)^{2 m}\left(\begin{array}{c}
Q-1-p \\
2 m
\end{array}\right)}{\left(1+q^{2(m+p)}\right)\left(1+q^{2(1+m+p)}\right)}
$$

38 Upon obtaining the hypergeometric functions, we make use of the following identities for $p \geqslant 0$ :

$$
\begin{aligned}
& \sum_{m=0}^{Q}(-1)^{m} q^{-m}(1+q)^{2 m}\left(\begin{array}{c}
Q+m \\
2 p+2 m
\end{array}\right)=\left(\begin{array}{c}
Q \\
2 p
\end{array}\right){ }_{3} F_{2}\left[\begin{array}{c}
1, Q+1,2 p-Q \\
p+1 / 2, p+1
\end{array} ; \frac{(1+q)^{2}}{4 q}\right] \\
& \sum_{m=0}^{Q}(-1)^{m} q^{-m}(1+q)^{2 m+1}\left(\begin{array}{c}
Q+m \\
1+2 p+2 m
\end{array}\right)=\left(\begin{array}{l}
Q 2 p+1 \\
)_{3} F_{2}\left[\begin{array}{c}
1, Q+1,2 p+1-Q \\
p+1, p+3 / 2
\end{array} ; \frac{(1+q)^{2}}{4 q}\right] .
\end{array}\right.
\end{aligned}
$$




$$
w_{2}(q, Q)=q^{-\frac{9}{2}} \sum_{m=0}^{\infty} \sum_{p=0}^{Q-1} \frac{(-1)^{p+1} q^{2\left(m+p+\frac{3}{2}\right)^{2}-2 m}\left(1+q^{2}\right)^{2 m+1}\left(\begin{array}{c}
Q-1-p \\
1+2 m
\end{array}\right)}{\left(1+q^{1+2 m+2 p}\right)\left(1+q^{3+2 m+2 p}\right)} .
$$

Combining the three sets of summations into (4.62), we have

$$
\begin{aligned}
& G_{2 n+1,2 Q}(q) \\
& =\frac{(1-q)^{2} q^{n}}{2 q^{\frac{3}{2}}(q ; q)_{\infty}^{6}}\left\{\vartheta _ { 2 } ( 1 , q ) ^ { 2 } \left[q^{1-Q_{u}}(\sqrt{q}, Q)\right.\right. \\
& \left.+(-1)^{Q}(1-q)\left(v_{1}(\sqrt{q}, Q)+q^{-1 / 4} w_{1}(\sqrt{q}, Q)\right)\right] \\
& +\vartheta_{3}(1, q)^{2}\left[-q^{1-Q_{u_{2}}}(\sqrt{q}, Q)+(-1)^{Q}(1-q)\left(v_{2}(\sqrt{q}, Q)+q^{2} w_{2}(\sqrt{q}, Q)\right)\right] \\
& +\vartheta_{4}(1, q)^{2}\left[q^{1-Q_{u}}(-\sqrt{q}, Q)-(-1)^{Q}(1-q)\left(v_{2}(-\sqrt{q}, Q)\right.\right. \\
& \left.\left.\left.+q^{2} w_{2}(-\sqrt{q}, Q)\right)\right]\right\}
\end{aligned}
$$

which exactly (4.63) with the definition (4.65) for the function $\mathcal{F}(q, Q)$ in the curly brackets. Note that this formula reproduces (4.72) when $Q=0$.

This allows to quickly infer asymptotic $\llbracket 2 n, 2 Q+1 \rrbracket$ multiplicities through the recursion (4.38) and the asymptotic relations $G_{2 n+2,2 Q+1}(q) \sim q G_{2 n, 2 Q+1}(q)$ as $n \rightarrow \infty$ :

$$
G_{2 n, 2 Q+1}(q) \sim \frac{1}{1+q}\left[F_{n, Q+1}^{\mathrm{R}}(q)-G_{2 n+1,2 Q}(q)-G_{2 n+1,2 Q+2}(q)\right] .
$$

The asymptotic formula (4.28) for $F_{n, Q+1}^{\mathrm{R}}(q)$ and the definition (4.65) for the function $\mathcal{F}(q, Q)$ then leads to (4.64).

\section{Appendix D. Supplementary material}

Supplementary material related to this article can be found online at http://dx.doi.org/10.1016/ j.nuclphysb.2013.08.003.

\section{References}

[1] K. Narain, M. Sarmadi, E. Witten, A note on toroidal compactification of heterotic string theory, Nucl. Phys. B 279 (1987) 369.

[2] L.A. Anchordoqui, I. Antoniadis, H. Goldberg, X. Huang, D. Lüst, et al., Vacuum stability of Standard Model ${ }^{++}$, arXiv:1208.2821 [hep-ph].

[3] W.-Z. Feng, D. Lüst, O. Schlotterer, S. Stieberger, T.R. Taylor, Direct production of lightest Regge resonances, Nucl. Phys. B 843 (2011) 570-601, arXiv:1007.5254 [hep-th].

[4] I. Antoniadis, N. Arkani-Hamed, S. Dimopoulos, G. Dvali, New dimensions at a millimeter to a Fermi and superstrings at a TeV, Phys. Lett. B 436 (1998) 257-263, arXiv:hep-ph/9804398.

[5] D. Lust, S. Stieberger, T.R. Taylor, The LHC string Hunter's companion, Nucl. Phys. B 808 (2009) 1-52, arXiv:0807.3333 [hep-th].

[6] D. Lust, O. Schlotterer, S. Stieberger, T. Taylor, The LHC string Hunter's companion (II): Five-particle amplitudes and universal properties, Nucl. Phys. B 828 (2010) 139-200, arXiv:0908.0409 [hep-th].

[7] A. Sagnotti, M. Taronna, String lessons for higher-spin interactions, Nucl. Phys. B 842 (2011) 299-361, arXiv:1006.5242 [hep-th].

[8] T.L. Curtright, C.B. Thorn, Symmetry patterns in the mass spectra of dual string models, Nucl. Phys. B 274 (1986) 520 .

[9] A. Hanany, D. Forcella, J. Troost, The covariant perturbative string spectrum, Nucl. Phys. B 846 (2011) 212-225, arXiv:1007.2622 [hep-th]. 
[10] B. Feng, A. Hanany, Y.-H. He, Counting gauge invariants: the plethystic program, J. High Energy Phys. 0703 (2007) 090, arXiv:hep-th/0701063.

[11] S. Benvenuti, B. Feng, A. Hanany, Y.-H. He, Counting BPS operators in gauge theories: Quivers, syzygies and plethystics, J. High Energy Phys. 0711 (2007) 050, arXiv:hep-th/0608050.

[12] D. Forcella, A. Hanany, Y.-H. He, A. Zaffaroni, The master space of $N=1$ gauge theories, J. High Energy Phys. 0808 (2008) 012, arXiv:0801.1585 [hep-th].

[13] J. Gray, A. Hanany, Y.-H. He, V. Jejjala, N. Mekareeya, SQCD: A geometric apercu, J. High Energy Phys. 0805 (2008) 099, arXiv:0803.4257 [hep-th].

[14] A. Hanany, N. Mekareeya, Counting gauge invariant operators in SQCD with classical gauge groups, J. High Energy Phys. 0810 (2008) 012, arXiv:0805.3728 [hep-th].

[15] A. Hanany, N. Mekareeya, A. Zaffaroni, Partition functions for membrane theories, J. High Energy Phys. 0809 (2008) 090, arXiv:0806.4212 [hep-th].

[16] S. Benvenuti, A. Hanany, N. Mekareeya, The Hilbert series of the one instanton moduli space, J. High Energy Phys. 1006 (2010) 100, arXiv:1005.3026 [hep-th].

[17] A. Hanany, N. Mekareeya, Tri-vertices and SU(2)'s, J. High Energy Phys. 1102 (2011) 069, arXiv:1012.2119 [hepth].

[18] A. Hanany, N. Mekareeya, S.S. Razamat, Hilbert series for moduli spaces of two instantons, arXiv:1205.4741 [hep-th].

[19] M.C. Cheng, K3 surfaces, $N=4$ dyons, and the Mathieu group M24, Commun. Number Theory Phys. 4 (2010) 623-658, arXiv:1005.5415 [hep-th].

[20] M.R. Gaberdiel, S. Hohenegger, R. Volpato, Mathieu twining characters for K3, J. High Energy Phys. 1009 (2010) 058, arXiv:1006.0221 [hep-th].

[21] M.R. Gaberdiel, S. Hohenegger, R. Volpato, Mathieu Moonshine in the elliptic genus of K3, J. High Energy Phys. 1010 (2010) 062, arXiv:1008.3778 [hep-th].

[22] T. Eguchi, K. Hikami, Note on twisted elliptic genus of K3 surface, Phys. Lett. B 694 (2011) 446-455, arXiv:1008.4924 [hep-th].

[23] S. Govindarajan, Unravelling Mathieu Moonshine, arXiv:1106.5715 [hep-th].

[24] M.C. Cheng, J.F. Duncan, J.A. Harvey, Umbral Moonshine, arXiv:1204.2779 [math.RT].

[25] M.B. Green, J. Schwarz, E. Witten, Superstring Theory, vol. 1: Introduction, 1987.

[26] M.B. Green, J. Schwarz, E. Witten, Superstring Theory, vol. 2: Loop Amplitudes, Anomalies and Phenomenology, 1987.

[27] M. Berkooz, M.R. Douglas, R.G. Leigh, Branes intersecting at angles, Nucl. Phys. B 480 (1996) 265-278, arXiv:hep-th/9606139.

[28] C. Bender, S. Orszag, Advanced Mathematical Methods for Scientists and Engineers: Asymptotic Methods and Perturbation Theory, Advanced Mathematical Methods for Scientists and Engineers, Springer, 1978.

[29] G. Hardy, E. Wright, An Introduction to the Theory of Numbers, Oxford Science Publications, Clarendon Press, 1979.

[30] T. Banks, L.J. Dixon, D. Friedan, E.J. Martinec, Phenomenology and conformal field theory or can string theory predict the weak mixing angle? Nucl. Phys. B 299 (1988) 613-626.

[31] T. Banks, L.J. Dixon, Constraints on string vacua with space-time supersymmetry, Nucl. Phys. B 307 (1988) 93-108.

[32] S. Ferrara, D. Lüst, S. Theisen, World sheet versus spectrum symmetries in heterotic and Type II superstrings, Nucl. Phys. B 325 (1989) 501.

[33] W.-Z. Feng, D. Lüst, O. Schlotterer, Massive supermultiplets in four-dimensional superstring theory, Nucl. Phys. B 861 (2012) 175-235, arXiv:1202.4466 [hep-th].

[34] S. Odake, Character formulas of an extended superconformal algebra relevant to string compactification, Int. J. Mod. Phys. A 5 (1990) 897.

[35] T. Eguchi, A. Taormina, On the unitary representations of $N=2$ and $N=4$ superconformal algebras, Phys. Lett. B 210 (1988) 125.

[36] T. Eguchi, K. Hikami, Superconformal algebras and Mock theta functions, J. Phys. A 42 (2009) 304010 , arXiv:0812.1151 [math-ph].

[37] T. Eguchi, K. Hikami, Superconformal algebras and Mock theta functions 2. Rademacher expansion for K3 surface, arXiv:0904.0911 [math-ph].

[38] R.H. Boels, C. Schwinn, On-shell supersymmetry for massive multiplets, Phys. Rev. D 84 (2011) 065006 , arXiv:1104.2280 [hep-th].

[39] N. Berkovits, M.M. Leite, First massive state of the superstring in superspace, Phys. Lett. B 415 (1997) 144-148, arXiv:hep-th/9709148 
[40] N. Berkovits, M.M. Leite, Superspace action for the first massive states of the superstring, Phys. Lett. B 454 (1999) 38-42, arXiv:hep-th/9812153.

[41] S. Zwegers, Mock theta functions, PhD thesis, Utrecht University, 2002, arXiv:0807.4834 [math-NT].

[42] R.H. Boels, D. O’Connell, Simple superamplitudes in higher dimensions, arXiv:1201.2653 [hep-th].

[43] R.H. Boels, Three particle superstring amplitudes with massive legs, J. High Energy Phys. 1206 (2012) 026 , arXiv:1201.2655 [hep-th].

[44] R.H. Boels, O. Schlotterer, in preparation, arXiv:12XX.YYYY [hep-th].

[45] B. de Wit, D. Lüst, BPS amplitudes, helicity supertraces and membranes in M theory, Phys. Lett. B 477 (2000) 299-308, arXiv:hep-th/9912225.

[46] J.M. Maldacena, The large $N$ limit of superconformal field theories and supergravity, Adv. Theor. Math. Phys. 2 (1998) 231-252, arXiv:hep-th/9711200.

[47] O. Aharony, S.S. Gubser, J.M. Maldacena, H. Ooguri, Y. Oz, Large $N$ field theories, string theory and gravity, Phys. Rep. 323 (2000) 183-386, arXiv:hep-th/9905111.

[48] S. Gerigk, String states on $A d S_{3} \times S^{3}$ from the supergroup, arXiv:1208.0345 [hep-th].

[49] L. Mazzucato, Superstrings in AdS, arXiv:1104.2604 [hep-th].

[50] M. Bianchi, J.F. Morales, H. Samtleben, On stringy $A d S_{5} \times S^{5}$ and higher spin holography, J. High Energy Phys. 0307 (2003) 062, arXiv:hep-th/0305052.

[51] M.R. Gaberdiel, R. Gopakumar, A. Saha, Quantum W-symmetry in $A d S_{3}$, J. High Energy Phys. 1102 (2011) 004 , arXiv:1009.6087 [hep-th].

[52] M.R. Gaberdiel, R. Gopakumar, An $A d S_{3}$ dual for minimal model CFTs, Phys. Rev. D 83 (2011) 066007 , arXiv:1011.2986 [hep-th].

[53] M.R. Gaberdiel, R. Gopakumar, T. Hartman, S. Raju, Partition functions of holographic minimal models, J. High Energy Phys. 1108 (2011) 077, arXiv:1106.1897 [hep-th].

[54] E. Kiritsis, Introduction to superstring theory, arXiv:hep-th/9709062. 